Portland State University

PDXScholar

Spring 6-7-2019

\title{
Application of Single Particle Electron Microscopy to Native Lens Gap Junctions and Intrinsically Disordered Signaling Complexes
}

Janette Bernadette Myers

Portland State University

Follow this and additional works at: https://pdxscholar.library.pdx.edu/open_access_etds

Part of the Biochemistry Commons, and the Molecular Biology Commons Let us know how access to this document benefits you.

\section{Recommended Citation}

Myers, Janette Bernadette, "Application of Single Particle Electron Microscopy to Native Lens Gap Junctions and Intrinsically Disordered Signaling Complexes" (2019). Dissertations and Theses. Paper 5008.

https://doi.org/10.15760/etd.6884

This Dissertation is brought to you for free and open access. It has been accepted for inclusion in Dissertations and Theses by an authorized administrator of PDXScholar. Please contact us if we can make this document more accessible: pdxscholar@pdx.edu. 
Application of Single Particle Electron Microscopy to Native Lens Gap Junctions and Intrinsically Disordered Signaling Complexes

\title{
by
}

Janette Bernadette Myers

A dissertation submitted in partial fulfillment of the requirements for the degree of

\author{
Doctor of Philosophy \\ in \\ Chemistry
}

\author{
Dissertation Committee: \\ Steve Reichow \\ John Perona \\ David Peyton \\ Dirk Iwata-Reuyl \\ Michael Bartlett
}

Portland State University

2019 


\section{Abstract}

Gap junctions are a class of membrane proteins that facilitate cell-to-cell communication by forming channels that directly couple the cytoplasm of neighboring cells. The channels are composed of monomers called connexins. Humans express 21 connexin isoforms in a cell-type specific fashion, and each isoform has distinct mechanisms of permeation and regulation. Co-assembly of multiple isoforms into a single intercellular channel can change channel properties, such as conductance and selectivity to substrates (e.g., ions, metabolites and signaling molecules). However, the mechanistic basis for this functional diversity has remained poorly understood. This lack of mechanistic insight has been due in large part to the lack of high-resolution (atomic-level) structural knowledge on this class of proteins. Prior to this work, the only highresolution information available on gap junction structure came from a single connexin isoform, connexin-26 (cx26).

CryoEM has recently transformed from a low-resolution technique into one capable of rivaling the atomic-level resolutions achieved by x-ray crystallography - but without the necessity for crystal formation, which has hindered progress towards understanding many classes of proteins (ie, membrane proteins, intrinsically disordered cell signaling complexes and other structurally dynamic systems). For my thesis research, I applied novel methods in single particle electron cryo-microscopy (CryoEM) to study a class of membrane proteins called gap junctions isolated from native lens tissue, as well as two signaling complexes 
not amenable to other structural techniques. I determined the structure of the lens gap junction, which contains connexin-46 (cx46) and connexin-50 (cx50), to a resolution of $3.4 \AA$ and generated atomic models for both connexin isoforms. Structural analysis paired with molecular dynamics gave insight into energetic features of these channels that determine their isoform-specific conductance and selectivity to electrically charged ions. The cx46/50 gating domain was found to be stabilized by hydrophobic anchors, and also seems to adopt a more stable open state than found in cx26. Genetic mutations associated with congenital cataract formation were found to map to hot-spots of conserved structural and functional importance, rationalizing their disease-causing effects.

As part of collaborative efforts, I used methods in single particle EM to characterize two separate signaling complexes that had proven difficult to study with $\mathrm{x}$-ray crystallography and NMR spectroscopy. One system, $\mathrm{Ca}^{2+/}$ Calmodulin Kinase II (CaMKII), is a signaling complex in the brain involved in memory formation. Characterization of the CaMKII complex by single particle EM revealed an extended state, which was also shown to be prevalent in cells giving more depth to our understanding of how this signaling molecule functions. The second collaboration characterized the multimeric binding sites of the hub protein LC8, which interacts with the disordered region of a transcription factor (ASCIZ). This provided support for a novel model of transcription regulation, wherein LC8 fine-tunes its own transcription levels through multi-valent binding to the disordered region of its own regulatory transcription factor. 


\section{Table of Contents}

Abstract

List of Tables

iv

List of Figures

V

\section{Chapter 1}

Introduction to CryoEM and the Resolution Revolution

1

Chapter 2

Gap Junction Structure, Function and Regulation

Chapter 3

Structural Characterization of Native Lens Connexin-46/50 Gap Junction

Channels in the open state by CryoEM

Chapter 4

The CaMKII holoenzyme structure in activation-competent conformations

Chapter 5

Multivalency regulates activity in an intrinsically disordered transcription factor

Chapter 6

The Future of in situ Cryo-Electron Microscopy

Appendix A. Full-length Gap Junction Purification Protocol

Appendix B. Signal Subtraction Protocol

Appendix C. List of Supplemental Files 


\section{List of Tables}

\section{Chapter 3}

Supplementary Table 1. Summary of molecular dynamics simulation setup and conditions for Cx50, Cx46, Cx26 and Cx46/50 heteromeric/heterotypic models

Supplementary Table 2. Local resolution assessment of a calculated map based on the Cx46 atomic model versus the $3.4 \AA$ CryoEM density map Supplementary Table 3. Local resolution assessment of a calculated map 104 based on the Cx50 atomic model versus the $3.4 \AA$ CryoEM density map

\section{Chapter 4}

Supplementary Table 1. Molecular crowding with $150 \mathrm{mg} / \mathrm{ml}$ BSA does not increase the Hill coefficient for mammalian-expressed CaMKIla Supplementary Table 2. Molecular crowding with $150 \mathrm{mg} / \mathrm{ml} \mathrm{BSA} \mathrm{might}$ 171 induce a compact conformation for linker-less (LL) CaMKIla

\section{Chapter 5}

Table 1. Thermodynamic parameters of dASCIZ-LC8 interactions

Table 2. Thermodynamic parameters of peptide-LC8 interactions

Table 3. Thermodynamic parameters of ASCIZ-LC8 interactions 


\section{List of Figures}

\section{Chapter 1}

Figure 1. The number of maps deposited to the Electron Microscopy 3

Databank at better than $4 \AA$ resolution by year.

Figure 2. Schematic of single particle cryoEM data acquisition

Figure 3. Advantages and limitations of negative stain compared to cryoEM 13 sample preparation techniques

Figure 4. Collection of micrograph "movies" allows for correction of beam- 15 induced motion.

\section{Chapter 2}

Figure 1. The level of detail in gap junction models has increased over time.

Figure 2. Co-assembly of connexin isoforms can produce a variety of mixed channels.

Figure 3. Gap junctions play a key role in the lens circulation system

\section{Chapter 3}

Figure 1. Structure of Cx46/50 intercellular channels

Figure 2. Heteromeric and heterotypic interactions between $\mathrm{Cx} 46$ and Cx50

Figure 3. Comparative pore profile and energetics of ion permeation.

Figure 4. Open-state stabilization of the $\mathrm{Cx} 46 / 50 \mathrm{NTH}$ domain.

Figure 5. Mutational hot spots in $\mathrm{Cx} 46$ and $\mathrm{C} \times 50$ linked to congenital cataracts.

Extended Data Figure 1. Preliminary structural analysis of native lens

Extended Data Figure 2. Overview of cryo-EM image processing and

3D reconstruction

Extended Data Figure 3. Global and local resolution analysis

Extended Data Figure 4. Cx46 and Cx50 atomic models fit to the cryo-EM 
$\mathrm{K}^{+} / \mathrm{Cl}^{-}$PMFs resulting from $\mathrm{N}$-terminal acetylation and $\mathrm{Cx} 46 / 50$

heterotypic/heteromeric assembly

Extended Data Figure 9. Analysis of $\mathrm{K}^{+}$trajectories and putative binding 98

site observed during molecular dynamics simulation

Extended Data Figure 10. Dynamic hydrogen-bond network within

100

the NTH domain observed by molecular dynamics simulation

\section{Chapter 4}

Figure 1. An extended form of the CaMKIla holoenzyme resolved by single 123 particle EM

Figure 2. 3D-reconstruction and pseudo-atomic model of the CaMKIla holoenzyme

Figure 3. Structural diversity of CaMKIla kinase domain arrangements

measured from single particle images.

Figure 4. Putative kinase domain pairing in the CaMKlla holoenzyme observed by single particle EM.

Figure 5. Full-length CaMKIla wild type expressed in eukaryotic cells

remains largely in an extended conformation even during molecular crowding.

Figure 6. A FRET-based assay indicated a compact conformation within cells only for linker-less but not for full-length CaMKIla wild type.

Figure 7. Overview of CaMKIla kinase domain arrangements

Supplemental Figure 1. Overview of 2D classification routines and analysis

Supplemental Figure 2. Vitrified CaMKIla particles imaged by electron

Supplemental Figure 3. Focused 2D classification of hub complex

Supplemental Figure 4. Focused 2D classification of kinase domain

arrangements

Supplemental Figure 5. Workflow of 3D reconstruction and pseudo-atomic modeling of the CaMKIla holoenzyme.

Supplemental Figure 6. CaMKIla activation by $\mathrm{Ca}^{2+} / \mathrm{CaM}$ in vitro and the effects of molecular crowding (by $150 \mathrm{mg} / \mathrm{ml}$ BSA or lysozyme), a linkerless deletion mutant (LL; described to favor the compact conformation), and an I321E mutant (described to prevent the compact conformation). Supplemental Figure 7. A FRET-based assay indicated a compact conformation within cells only for linker-less but not for full-length CaMKIla wild type 


\section{Chapter 5}

Figure 1. LC8 dimerizes its protein partners 183

Figure 2. Domain structures of dASCIZ 187

Figure 3. LC8-dLBD interactions monitored by ITC 189

Figure 4. ASCIZ and LC8 form a dynamic complex with low occupancy 196 intermediates

Figure 5. dLBD:LC8 and hLBD:LC8 complexes visualized by negative stain 200 electron microscopy

Figure 6. NMR titration of the dLBD with LC8.

203

Figure 7. The number of LC8 recognition motifs tunes ASCIZ 206 transcriptional activity

Figure 8. Model of ASCIZ regulation of LC8 transcription 215

Figure 2 supplement 1. dLBD C-terminus is transiently structured 229

Figure 4 supplement 1. Primary SAXS data 229

Figure 5 supplement 1 . Single particle images of dLBD:LC8 and hLBD:LC8 230 complexes

Figure 6 supplement 1. Representative HNCO slices of dLBD titration with LC8

Figure 7 supplement 1 . Western blot of ASCIZ constructs 


\section{Chapter 1}

\section{Introduction to CryoEM and the Resolution Revolution}

Structural biology aims to understand cellular function at a cellular and molecular level. Efforts to determine the structure of individual proteins or macromolecular complexes compose a large portion of the field. Traditionally, techniques such as x-ray crystallography and NMR spectroscopy have been the default tools to determine macromolecular structures. With the capability of producing sufficiently high-resolution data to allow the building of atomic models, both techniques have provided a wealth of information to the field, illustrated by the sheer number of protein structure models deposited into the Protein Data Bank (PDB). Recently, however, an alternative technique called cryo-electron microscopy (cryoEM) experienced a "resolution revolution" - meaning cryoEM can now achieve nearatomic resolution, something previously restricted to crystallography and NMR. This breakthrough was driven by a combination of technological advances in camera technology, computational image processing algorithms and novel microscope design. ${ }^{1}$

As a result of this "resolution revolution", the popularity of cryoEM increased rapidly. ${ }^{1,2}$ In addition to the ability to produce high-resolution data, cryoEM can access systems not amenable to the traditional methods of x-ray crystallography and NMR. Crystallography, for example, requires the formation of ordered crystals - meaning that membrane proteins and heterogeneous samples are at best difficult and at worst impossible targets for crystallographers. Solution-state 
NMR spectroscopy, on the other hand, is size limited - it typically is not applied to systems much larger than $\sim 50 \mathrm{kDa}$, since technical limitations present an obstacle for larger complexes. Both $\mathrm{x}$-ray crystallography and NMR require large amounts of purified protein, which prevents the application of these techniques to targets that are difficult to express and purify.

Before the "resolution revolution" in 2013, cryoEM was limited to a resolution of approximately $15 \AA$, with notable exceptions for special cases like large, highly symmetric viruses ${ }^{1}$. Possessing both large size and a high degree of symmetry, virus particles were the first systems to reach near-atomic resolution by cryoEM. In fact, before 2013 , the only cryoEM data available at resolutions better than $4 \AA$ were from viruses, the first of which was Cytoplasmic Polyhedrosis Virus (determined in 2008) $)^{3} .2013$ saw the determination of the ion channel TRPV1 ${ }^{4}$ and 20 s proteaseome ${ }^{5}$ at better than $4 \AA$ resolution (Figure 1). Significantly smaller and with lower symmetry than a virus, these structures represented an important advance for the field. The determination of a ribosome structure at 3.2 $\AA$ resolution in $2014^{6}$ represented another milestone, impressive due to the ribosome's lack of internal symmetry. The number of maps deposited at high resolution increased rapidly after these breakthroughs in 2013 (see Figure 1). Notably, there were 27 EM density maps at better than $4 \AA$ resolution in EMDB in 2013; at the time of this writing the number of maps at better than $4 \AA$ resolution has grown to over 600 . 


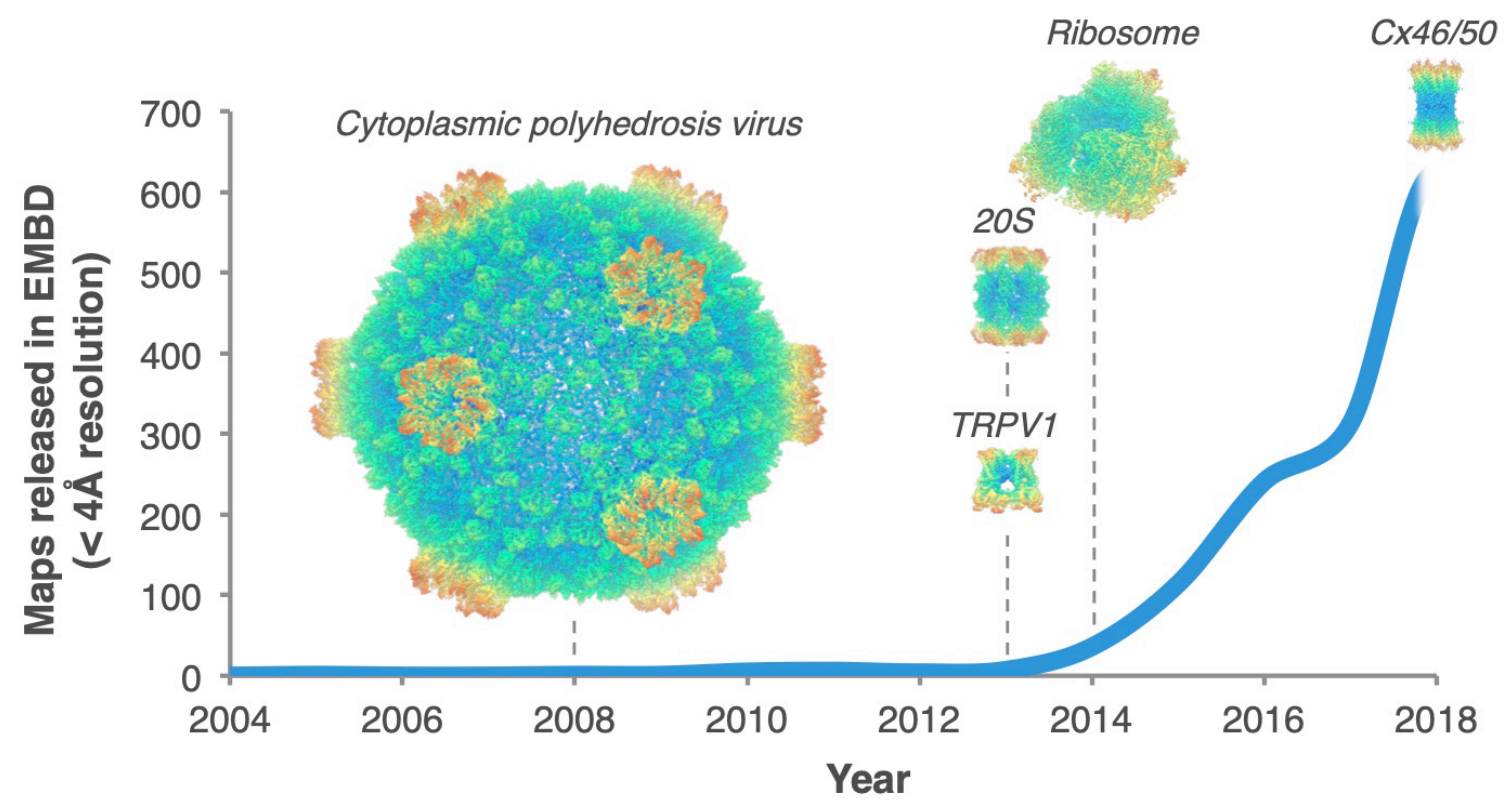

Figure 1. The number of maps deposited to the Electron Microscopy Databank at better than $4 A$ resolution by year. The number of maps sharply increases after 2013. Maps shown are to scale. Cytoplasmic polyhedrosis virus (EMD 1508) was determined at $3.88 \AA^{3}$, TRVP1 (EMD 5778) at $3.4 \AA^{4}$, 20S proteasome (EMD 5623) at $3.3 \AA^{15}$, and the ribosome structure (EMD 2566) at $3.2 \AA^{38}$.

Now that cryoEM can reach near atomic-resolution, it can be applied to investigate systems that have been difficult (or even impossible) to approach by crystallography and NMR spectroscopy. Membrane proteins are one such system - since, for cryoEM, the formation of crystals is unnecessary. One of the first proteins solved at near atomic resolution by cryoEM - the TRPV1 channel is a membrane protein channel. ${ }^{4}$ Membrane proteins represent a great opportunity for structural studies, since membrane proteins compose $\sim 30 \%$ of the proteome, yet only make up only $\sim 3.5 \%$ of the structures deposited to the PDB. $^{7}$ At the time of this writing, $\sim 20 \%$ of cryoEM structures deposited to the PDB are of membrane proteins, whereas only $\sim 3 \%$ of structures solved by 
crystallography or NMR spectroscopy are of membrane proteins - evidence that cryoEM can handle membrane proteins effectively.

CryoEM analysis of the ribosome also demonstrates the utility of the technique. The ribosome is a structurally heterogeneous, asymmetric complex, and cryoEM can separate the different conformational states present in a single ribosome dataset and refine them independently ${ }^{8,9}$. In another illustrative case, since cryoEM requires relatively small amounts of sample, it was possible to determine a near-atomic resolution structure of tau filaments isolated from a single Alzheimer's patient ${ }^{10}$. Working with large and highly symmetric proteins or complexes (e.g., viruses) is also no longer a requirement for cryoEM analysis the small ( $\mathrm{mw} \sim 170 \mathrm{kDa}$ ), asymmetric protein $\mathrm{\gamma}$-secretase has been determined by cryoEM at near-atomic resolution ${ }^{11}$, and glutamate dehydrogenase (mw 334 $\mathrm{kDa}$ ) at the remarkable resolution of $1.8 \mathrm{~A}^{12}$. At such resolutions, cryoEM can visualize small molecule ligands interacting with proteins, and therefore contribute drug design efforts. Notably, membrane proteins make up $\sim 60 \%$ of the current FDA approved targets ${ }^{13}$.

Cryo-electron microscopy overcame its previous limitations due to the convergence of multiple technological advances. Advances in sample preparation techniques ${ }^{14}$, camera technology ${ }^{15,16}$, and data processing software ${ }^{17-19}$ all contributed, culminating in the Nobel Prize in Chemistry for 2017 
being awarded to the pioneers of these techniques: Jaques Dubochet, Joachim Frank, and Richard Henderson ${ }^{20}$.

\section{Overview of CryoEM methodologies}

Multiple techniques fall under the umbrella of electron cryo-microscopy, each with their own advantages, disadvantages and limitations. These methods are 2D crystallography, tomography, and single particle cryoEM, each of which will be briefly described.

1) Electron Crystallography. 2D electron crystallography shares some characteristics with x-ray crystallography. Much like x-ray crystallography, diffraction patterns are collected - in this case from thin two-dimensional (2D) ordered crystalline specimens - and analyzed by Fourier-transform techniques. This methodology fills a niche for the study of membrane proteins, which in special circumstances may be coaxed into forming single-layered protein-lipid crystals $^{21}$. The first structure determined at near-atomic resolution by $2 \mathrm{D}$ crystallography was of bacteriorhodopsin (3.5 $\AA$ resolution), by Richard Henderson in $1990^{22}$. This structure achieved high resolution through the averaging of many copies of bacteriorhodopsin - in this case through the $2 \mathrm{D}$ crystal lattice. The primary advantage of $2 \mathrm{D}$ crystallography is that it allows the study of membrane protein structures in a lipid bilayer, for example the structure of aquaporin- $0^{23}$ and of the light harvesting complex ${ }^{24}$. The study of aquaporin-0 was an exceptional example, as this complex was resolved to $1.9 \AA$ resolution, 
and provided a detailed description of both the protein and lipid interactions that closely matched the features observed in native lens membranes ${ }^{23}$.

In other techniques, membrane proteins must be solubilized - primarily in detergents - to allow them to be isolated and studied. Removing a membrane protein from the lipid bilayer leads to legitimate concern over whether or not the detergent-solubilized structure is faithful to the lipid-solubilized structure found in cells. 2D crystallography avoids this caveat. However, forming 2D crystals is difficult. In fact, it's even harder to form a 2D crystal than 3D crystal - for 2D crystals, only 17 possible space groups exist ${ }^{25}$. The difficulty of forming 2D crystals is evidenced by relatively low success rate of $2 \mathrm{D}$ electron crystallography compared to traditional $\mathrm{x}$-ray crystallography. Additionally, some technical challenges exist in 2D crystallography that are absent in x-ray crystallography. 2D crystals must be tilted in order to obtain a 3D diffraction dataset. However, tilting results in anisotropic resolution, meaning that the resulting map has different resolutions on different axes relative to the beam. While 2D crystallography can attain near-atomic - or even atomic - resolution, difficulties with crystal formation limit this approach to a small number of targets.

2) Electron Tomography. The application of electron tomography has primarily been used as a method for imaging 3D cellular features and large macromolecular complexes in a native cellular environment. For this methodology, the thickness of cells must be reduced such that they are 
transparent to the electron beam $(\sim 100 \mathrm{~nm}$ or thinner). In most cases, this requires thinning of the cellular specimen, with different thinning techniques applied to fixed or frozen cells. These thin sections of cells are then imaged over a range of tilt angles, which allows reconstruction of the cellular volume. Traditionally, tomography employed fixatives such as heavy metal stains or embedded samples in plastic ${ }^{26}$. Modern methods, however, no longer require staining and are performed on vitrified specimens ${ }^{27}$. For example, a recent visualization of the periphery of the nuclear pore in a HeLa cell - accomplished with a remarkable level of detail - did not require the use of $\operatorname{stain}^{28}$. The greatest strength of tomography is that it allows imaging in a close to native context, which maintains the native context of the cell (e. g., cell crowding). Unfortunately, tomography is currently incapable of reaching near-atomic resolution detail, due in part to the accumulation of radiation damage caused by the electron beam during imaging. Additionally, the thinning and/or sectioning required for imaging may induce artifacts.

Recent advances in computational methods of sub-tomogram averaging have demonstrated that averaging of tomographic images can significantly improve the resolution of sub-cellular features. In sub-tomogram averaging, multiple copies of a target complex from a tomogram are averaged together, which increases the achievable resolution. This technique can now reach sub-nanometer resolutions, which when combined with other high-resolution structural knowledge can be 
quite powerful method for understanding the native state of complex assemblies $^{28-30}$.

3) Single particle CryoEM. This thesis will focus on single particle electron cryomicroscopy - which can now routinely reach sub-nanometer and near-atomic resolutions. In single particle EM, hundreds of thousands - or even millions - of individual particle images must be collected and averaged together; the 3D volume can then be reconstructed from these images.

To begin, purified protein is typically applied to specialized holey carbon grid (Figure 2). Rather than a continuous carbon support layer, these grids have a layer of carbon with holes of uniform size. After applying sample to the holey carbon grid, excess liquid is blotted away, leaving the sample suspended across the holes in a thin layer of buffer. The sample is then fixed by plunge freezing into

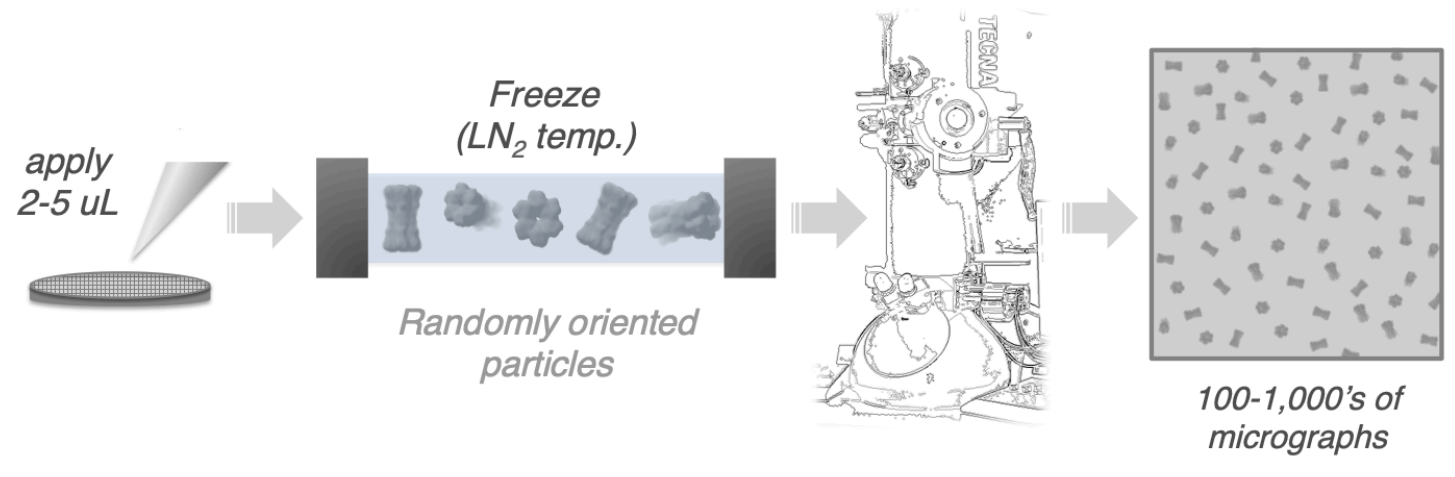

Figure 2. Schematic of single particle cryoEM data acquisition. A small volume of purified sample is applied to a holey carbon grid, then plunge frozen to produce a sample suspended in vitreous (electron transparent) ice. Grids are then imaged in a cryo microscope and large datasets are collected with automated software. 
liquid ethane, which rapidly freezes the specimen in amorphous ice. Ideally, this process results in the particles adopting random orientations in the ice, so that images of the particle can be obtained from many different angles. Grids must be screened for suitable particle distribution (i.e., not overlapping but still densely packed) and sufficiently thin ice before data collection. CryoEM datasets can contain anywhere from hundreds to thousands of micrographs, typically collected with semi-automated software. Individual particle images are selected and extracted from the micrographs - also a semi-automated process - and in good conditions a single micrograph can contain hundreds of individual particles. CryoEM datasets can range from 10,000's-1,000,000's of individual particle images depending on the sample.

Because the electron beam is transmitted through the specimen, each particle image represents a $2 \mathrm{D}$ projection of the $3 \mathrm{D}$ structure of the particle, containing information on both internal and external features. The 3D structure can be reconstructed from these $2 \mathrm{D}$ projections, provided we know some information about the orientation of the projection in 3D space. Successful reconstruction of a $3 \mathrm{D}$ volume requires the determination of $\mathrm{x}-\mathrm{y}$ shift and three rotation angles (Euler angles) that relate each individual particle to each other. Modern 3D reconstruction algorithms rely on the central section theorem of Fourier-transform analysis, which states that the Fourier transform of a $2 \mathrm{D}$ projection is a slice through the volume in 3D Fourier space. 
Some of the many advantages of single particle cryoEM include the fact that it requires a relatively small amount of protein (e.g., ng-ug), compared to the milligram quantities needed for x-ray crystallography and NMR spectroscopy. Furthermore, since crystallization is unnecessary, cryoEM is better suited to handle proteins that are resistant to crystallization (e.g., membrane proteins). Additionally, single particle EM can handle structural and/or compositional heterogeneity within the specimen - at least to an extent - since populations of different species can be classified computationally based on their structural differences.

\section{Historical limitations to achieving near-atomic resolution by CryoEM}

What, then, has limited the achievable resolution in cryoEM? Theoretically, cryoEM should reach $3 \AA$ for an asymmetrical, $\sim 100 \mathrm{kDa}$ particle even with a relatively small dataset of particles (e.g., $\sim 10,000$ particles) ${ }^{31}$. In practice, however, cryoEM requires larger datasets to reach near-atomic resolution (e.g., 100,000 or more, depending on symmetry). A few key factors account for cryoEM failing to reach this theoretical limit. One factor is radiation damage caused by the electron beam - an unavoidable consequence of imaging with electrons. Traditionally, embedding samples in heavy metal stain (e.g., Uranyl Formate) addressed the issue of damage - a technique called negative staining ${ }^{32}$ (Figure 3). The stain can withstand high doses of radiation, unlike the relatively fragile bonds in biological samples. The stain also provides improved contrast over the unstained sample, since the heavy atoms strongly scatter electrons. This means 
individual particles can be analyzed by negative stain. Paired with averaging and classification techniques, this high contrast can allow the investigation of heterogeneous samples that would be difficult even for $\mathrm{cryoEM}^{32}$. Unfortunately, the achievable resolution in negative stain is limited by the grain size of the stain and can only reach $\sim 15 \AA$ at best. Additionally, with this technique, the cast of the protein left behind in the stain is imaged rather than the protein itself - so internal information is lost. The staining procedure can also induce artifacts into the sample - most notably flattening of the resulting map by the stain.

Another important factor limiting achievable resolution is beam-induced movement of the EM grid. The grid inevitably moves when the electron beam interacts with it, inducing a drift that occurs over the course of the image exposure. A blurry image results - the details (i.e., high-resolution information) are lost. This problem is pronounced for biological samples, where radiation damage is also a concern. To minimize damage, a low dose of electrons are spread out over a long exposure time - anywhere from 3 to 30 seconds depending on the camera used. Since beam-induced movement is an unavoidable consequence of imaging with electrons, this problem seemed insurmountable.

Another challenge is that, since biological samples must be imaged with low doses of electrons to avoid radiation damage, the individual particle images extracted from unstained cryoEM micrographs have an extremely low signal-to- 
noise ratio (SNR). The low SNR of individual particles can be overcome by averaging together many particles in the same orientation, but in order to accomplish this, particle images need to be aligned to each other (i.e., classified) accurately. Older alignment algorithms required human intervention for classification of 2D images. These 2D class averages are often used to discard particles that the user has determined are not of good quality, or to separate populations in a heterogeneous dataset. However, it is difficult to assess heterogeneity from examining $2 \mathrm{D}$ projections or averages of projections. This could lead to a situation where, for example, a dataset contains particles with multiple conformational states that simply cannot be separated by the $2 \mathrm{D}$ classification algorithm. These issues with sample preparation, motion-induced blurring and particle alignment kept cryoEM from reaching near-atomic resolution for many years.

\section{Moving single particle CryoEM beyond the realm of "Blobology"}

Many historic and modern advances have ultimately contributed to the advancement of single particle CryoEM to its current high-resolution capability. One of the early advances was sample vitrification, or freezing. This preparation technique preserves the native (or near-native) structure of the sample. The vitrification technique was developed by Jacques Dubochet to image virus particles $^{14}$. Once applied to a grid, samples are plunged into liquid ethane, cooled to liquid nitrogen temperatures. Using this technique, freezing of the sample occurs rapidly enough to avoid the formation of crystalline ice - which would 
Negative Stain EM

- Radiation resistant

- High contrast

- Possible to analyze single particles

- Relatively simple specimen preparation

○ Limited to $\sim 15$ A resolution

- Image cast of particle

- Stain can induce artifacts

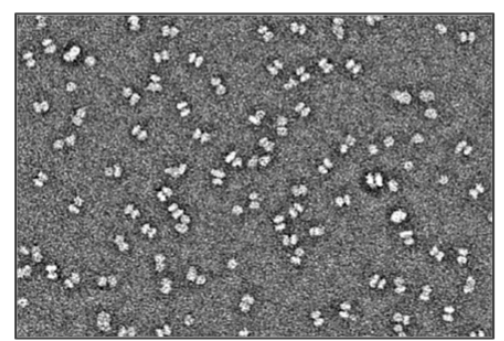

Negatively stained gap junctions

\section{CryoEM}

- Radiation sensitive

- Low contrast

- Need to average signal from many particles

- Technically challenging specimen preparation

- Can reach near-atomic resolution

- Image in near-native state preserved by vitreous ice

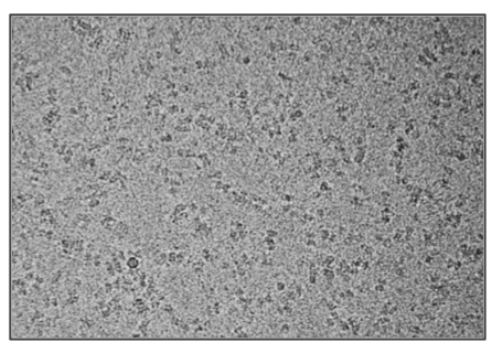

Unstained gap junctions in ice

Figure 3. Advantages and limitations of negative stain compared to cryoEM sample preparation techniques. Negatively stained and ice embedded gap junctions are provided as examples.

disrupt structure - and instead forms electron transparent vitreous ice. Freezing also protects against dehydration in the vacuum of the EM, though the system must be maintained at liquid nitrogen (i.e., cryo) temperatures. See Figure 3 for a comparison of the benefits of cryogenic sample preparation and negative stain. For overcoming the issue of low SNR in cryoEM images, advances in camera technology and image processing software were instrumental - namely the development of direct electron detectors. As implied, these cameras directly detect electrons without the need for a scintillator, as in CCD cameras ${ }^{33}$. Since they detect electrons directly, rather than requiring conversion into light by a scintillator before detection, direct detectors have a higher detective quantum efficiency (DQE) than CCD cameras ${ }^{16}$. In short, a camera with a higher DQE 
produces an image with a higher SNR compared to a detector with a lower DQE. In the case of direct detectors, the SNR is about twice that of film for when used for cryoEM ${ }^{34}$.

An unforeseen benefit of direct electron detectors was that they also helped tackle the issue of beam-induced motion blurring. Because of the high DQE, these cameras can collect image data in "movie" mode, which records multiframe movies instead of a single frame exposure. This "movie mode" allows for frame-by-frame correction of beam-induced motion and sample drift that accumulate during the total length of expsoure ${ }^{35}$. Recall that long exposures times at low dose rates are required in order to minimize radiation damage in biological cryoEM, and that this leads to sample drift over the time of exposure. The collection of "movies" allows specialized image processing software to correct for sample movement due to drift and beam induced charging ${ }^{18}$ (Figure 4). This key development allowed for the recovery of high-resolution information that would otherwise be lost due to motion ${ }^{33}$, and is one of the primary drivers behind the recent resolution revolution.

In addition to these advancements, more powerful algorithms for aligning single particle images have also been recently developed. It had already been established that different views/projections of particles could be classified using statistical methods - originally developed by Joachim Frank ${ }^{36,37}$. More recently, improved particle alignment algorithms based on Bayesian statistics (i.e., 
maximum likelihood methods) allowed for more robust classifications ${ }^{17}$. Rather than each particle being assigned a defined set of Euler angles, this more modern approach assigns each particle a weighted probability for being correctly assigned a given set of Euler angles. This tends to up-weight well assigned particles and down-weight particles with more ambiguous assignments, while still allowing these more ambiguous particles to contribute meaningfully to the reconstruction.

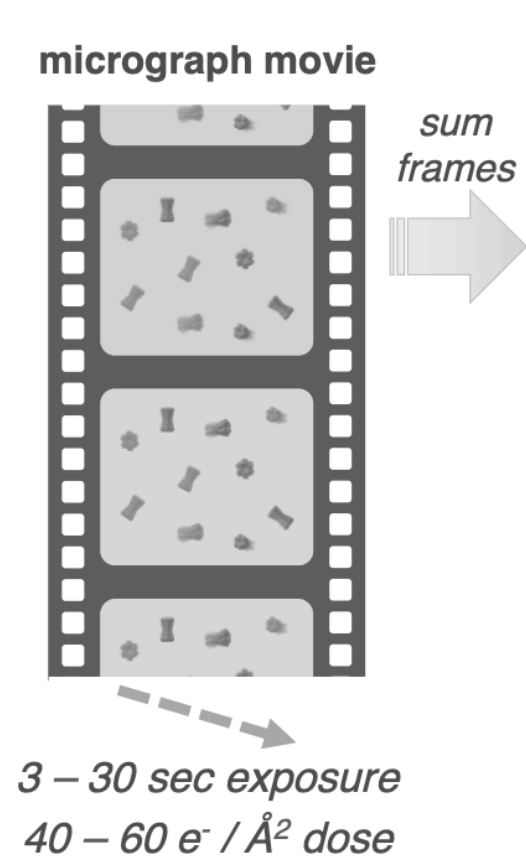

\begin{abstract}
uncorrected micrograph
\end{abstract}

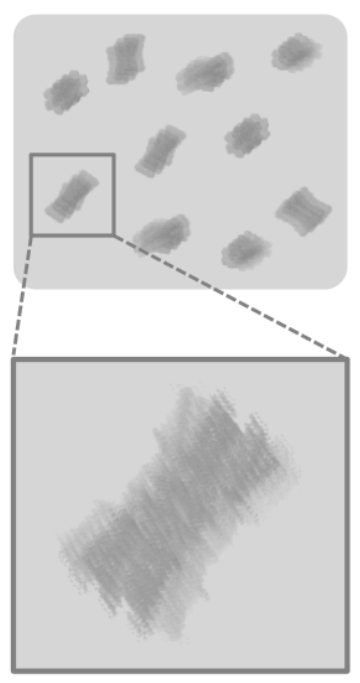

blurred motion-corrected micrograph
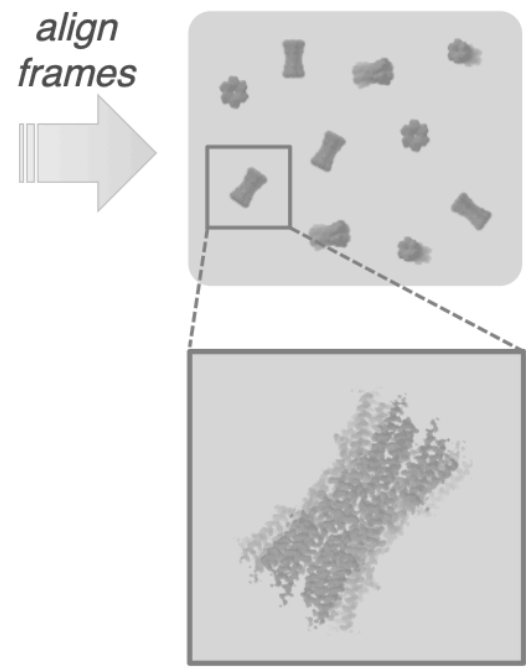

un-blurred

Figure 4. Collection of micrograph "movies" allows for correction of beam-induced motion. Many "frames" are collected over the course of the long exposure time required for biological EM. Without correcting for motion, the resulting image is blurred. After frame alignment, an "un-blurred" image is recovered and high-resolution information is restored. 
Additionally, recent algorithms have also been developed to perform classification in 3D space, in addition to 2D space. In terms of dissecting structural/compositional heterogeneity in a dataset, 3D classification is much more effective than 2D classification. Essentially, identifying different conformations of a complex by examining $3 \mathrm{D}$ maps is easier than trying to do the same by examining $2 \mathrm{D}$ projections. While this technique is used for all types of cryoEM data, it is especially helpful for heterogeneous datasets since it allows for more effective separation of different populations.

\section{Concluding Remarks}

Due to their contributions to the development of cryoEM as a bone-fide structural biology technique capable of reaching near-atomic resolution, Richard Hendersen, Joachim Frank and Jacques Dubochet were awarded the Nobel Prize in Chemistry in 2017. Thanks to these pioneers and to the recent technological advances, the field of cryoEM is experiencing an explosion in popularity, especially for targets that are difficult to approach for traditional methods.

For my thesis research, I have harnessed the powers of single particle CryoEM to characterize the structure and function of a class of membrane-embedded communication channels, called gap junctions. In order for multicellular organisms to survive, their cells need to communicate with each other. Cell communication takes many forms, and one form utilizes the gap junctions - a 
class of channels that directly link the cytoplasm of neighboring cells. The amount of structural information on the gap junctions has been limited, primarily because membrane proteins are difficult to study. However, this makes gap junctions an attractive target for cryoEM studies. For this work, I applied cryoEM to native gap junctions isolated from eye lens cells and solved the open structure at near-atomic resolution. A detailed background into the biology of this system is provided in Chapter 2, followed by my research using CryoEM to define the structure and key functional features of the lens gap junctions in Chapter 3.

In addition to this primary focus of my thesis research, I contributed to other collaborative works using single particle EM to characterize two intrinsically disordered signaling complexes. The first collaborative effort was performed with Prof. Ullrich Bayer at the University of Colorado, where I used single particle EM to characterize the Calcium/Calmodulin dependent protein kinase II (CaMKII), which is involved in memory formation and long-term potentiation in the brain (see Chapter 4). The second collaboration was performed with Prof. Elisar Barabar at Oregon State University, and characterized the interaction between the disordered region of a transcription factor (ASCIZ) and a ubiquitous hub protein called LC8, which binds to the transcription factor in a multivalent fashion to regulate its own expression level (see Chapter 5). In the concluding chapter of this thesis (Chapter 6), I provide a forward-looking analysis of the impact that the technological advances described may have on the field of cryo electron microscopy. 


\section{References}

1. Bai, X. chen, McMullan, G. \& Scheres, S. H. . How cryo-EM is revolutionizing structural biology. Trends in Biochemical Sciences 40, 4957 (2015).

2. Kühlbrandt, W. The resolution revolution. Science 343, 1443-1444 (2014).

3. Yu, X., Jin, L. \& Zhou, Z. H. 3.88 A Structure of Cytoplasmic Polyhedrosis Virus By Cryo-Electron Microscopy. Nature 453, 415-419 (2008).

4. Liao, M., Cao, E., Julius, D. \& Cheng, Y. Structure of the TRPV1 ion channel determined by electron cryo-microscopy. Nature 504, 107-12 (2013).

5. Li, X. et al. Electron counting and beam-induced motion correction enable near atomic resolution single particle cryoEM. Nat. Methods 10, 584-590 (2013).

6. Brown, A. et al. Structure of the large ribosomal subunit from human mitochondria. Science (80-. ). (2014). doi:10.1126/science.1258026

7. Almén, M., Nordström, K. J., Fredriksson, R. \& Schiöth, H. B. Mapping the human membrane proteome: a majority of the human membrane proteins can be classified according to function and evolutionary origin. BMC Biol. 7,50 (2009).

8. Lu, Y. et al. Conformational Landscape of the p28-Bound Human Proteasome Regulatory Particle. Mol. Cell 67, 322-333.e6 (2017).

9. Wong, W. et al. Cryo-EM structure of the Plasmodium falciparum $80 \mathrm{~S}$ ribosome bound to the anti-protozoan drug emetine. Elife (2014). 
doi:10.7554/eLife.03080

10. Fitzpatrick, A. W. P. et al. Cryo-EM structures of tau filaments from Alzheimer's disease. Nature (2017). doi:10.1038/nature23002

11. Bai, X. C. et al. An atomic structure of human y-secretase. Nature 525, 212-7 (2015).

12. Merk, A. et al. Breaking Cryo-EM Resolution Barriers to Facilitate Article Breaking Cryo-EM Resolution Barriers to Facilitate Drug Discovery. Cell 165, 1698-1707 (2016).

13. Dubochet, J., Frank, J. \& Henderson, R. Cryo-EM in drug discovery: achievements, limitations and prospects. Nat. Rev. Drug Discov. (2018). doi:10.1038/nrd.2018.77

14. Adrian, M., Dubochet, J., Lepault, A. \& McDowall, J. Cryo-electron microscopy of viruses. Nature (1984). doi:10.1111/febs.12078.Cryoelectron

15. Li, X. et al. Electron counting and beam-induced motion correction enable near-atomic-resolution single-particle cryo-EM. Nat. Methods 10, 584-590 (2013).

16. Ruskin, R. S., Yu, Z. \& Grigorieff, N. Quantitative characterization of electron detectors for transmission electron microscopy. J. Struct. Biol. 184, 385-393 (2013).

17. Scheres, S. H. W. RELION: Implementation of a Bayesian approach to cryo-EM structure determination. J. Struct. Biol. 180, 519-530 (2012).

18. Zheng, S. Q. et al. MotionCor2: anisotropic correction of beam-induced 
motion for improved cryo-electron microscopy. Nat. Methods 14, 331-332 (2017).

19. Zhang, K. Gctf: Real-time CTF determination and correction. J. Struct. Biol. 193, 1-12 (2016).

20. NobelPrize.org. The Nobel Prize in Chemistry 2017. at <https://www.nobelprize.org/prizes/chemistry/2017/summary/>

21. Fujiyoshi, Y. Electron crystallography for structural and functional studies of membrane proteins. J. Electron Microsc. (Tokyo). 60, 149-159 (2011).

22. Henderson, R. et al. Model for the structure of bacteriorhodopsin based on high-resolution electron cryo-microscopy. J. Mol. Biol. (1990).

doi:10.1016/S0022-2836(05)80271-2

23. Gonen, T. et al. Lipid-protein interactions in double-layered twodimensional AQP0 crystals. Nature (2005). doi:10.1038/nature04321

24. Kühlbrandt, W., Wang, D. N. \& Fujiyoshi, Y. Atomic model of plant lightharvesting complex by electron crystallography. Nature (1994). doi:10.1038/367614a0

25. Nannenga, B. L., ladanza, M. G., Vollmar, B. S. \& Gonen, T. Overview of electron crystallography of membrane proteins: Crystallization and screening strategies using negative stain electron microscopy. Curr. Protoc. Protein Sci. 1-11 (2013). doi:10.1002/0471140864.ps1715s72

26. Baumeister, W. Electron tomography: Towards visualizing the molecular organization of the cytoplasm. Current Opinion in Structural Biology (2002). doi:10.1016/S0959-440X(02)00378-0 
27. Wagner, J., Schaffer, M. \& Fernández-Busnadiego, R. Cryo-electron tomography-the cell biology that came in from the cold. FEBS Lett. 591, 2520-2533 (2017).

28. Mahamid, J. et al. Visualizing the molecular sociology at the HeLa cell nuclear periphery. Science (80-. ). (2016). doi:10.1126/science.aad8857

29. Kudryashev, M. et al. The Structure of the Mouse Serotonin 5-HT3 Receptor in Lipid Vesicles. Structure (2016). doi:10.1016/j.str.2015.11.004

30. Briggs, J. A. G. Structural biology in situ-the potential of subtomogram averaging. Curr. Opin. Struct. Biol. 23, 261-267 (2013).

31. Henderson, R. The potential and limitations of neutrons, electrons and Xrays for atomic resolution microscopy of unstained biological molecules. $Q$. Rev. Biophys. 28, 171-193 (1995).

32. Ohi, M., Li, Y., Cheng, Y. \& Walz, T. Negative Staining and Image Classification - Powerful Tools in Modern Electron Microscopy. Biol. Proced. Online 6, 23-34 (2004).

33. Li, X. et al. Electron counting and beam-induced motion correction enable near-atomic-resolution single-particle cryo-EM. Nat. Methods 10, 584-590 (2013).

34. Mcmullan, G., Faruqi, A. R., Clare, D. \& Henderson, R. Comparision of optimal performance at $300 \mathrm{keV}$ of three direct electron detectors for use in low dose electron microscopy. Ultramicroscopy 147, 156-163 (2014).

35. Campbell, M. G. et al. Movies of Ice-Embedded Particles Enhance Resolution in Electron Cryo-Microscopy. Structure 20, 1823-1828 (2012). 
36. Frank, J., Goldfarb, W., Eisenberg, D. \& Baker, T. S. Reconstruction of glutamine synthetase using computer averaging. Ultramicroscopy (1978). doi:10.1016/S0304-3991(78)80038-2

37. van Heel, M. \& Frank, J. Use of multivariates statistics in analysing the images of biological macromolecules. Ultramicroscopy (1981). doi:10.1016/S0304-3991(81)80197-0

38. Amunts, A. et al. Structure of the Yeast Mitochondrial Large Ribosomal Subunit. Microsc. Microanal. (2014). doi:10.1017/s1431927614007995 


\section{Chapter 2}

\section{Gap junction structure, function and regulation}

The gap junctions play an essential role in vertebrate species by facilitating cellto-cell communication ${ }^{1}$. They have important roles throughout the human body, especially in electrically coupled cells found in the heart and in the brain. Because of their importance to physiology, mutations in gap junctions are associated with a variety of disease states, indicating their importance. These include deafness ${ }^{2}$, myelin-related neurological disorders ${ }^{3}$, skin disorders ${ }^{4}$ and congenital cataracts ${ }^{5}$. Mutations in gap junctions are also known to be linked to a disorder called oculodendrodigital dysplasia (ODDD), which is associated with craniofacial deformity and other bone alterations, among other symptoms ${ }^{6}$. Lastly, gap junction misregulation is known to be involved in ischemic conditions following stroke and cardiac arrhythmia, as well as some cancers ${ }^{7,8}$. Because of their importance in a number of physiological phenomena, gap junctions have long been an attractive target for structural studies, since the results of these studies could aid in the development of targeted pharmacological treatments for disease.

Early characterizations of gap junctions were carried out using negative staining electron microscopy and x-ray diffraction. In 1977, gap junctions isolated from mouse liver cells were investigated using those techinques ${ }^{9}$. These studies showed that gap junctions formed 2D hexagonal arrays that assembled in large plaques within the native membrane. In 1984, also using rat liver gap junctions 
investigated by electron crystallography, investigators learned that individual gap junction intercellular channels are formed by the end to end docking of two hexameric structures "hemi-channels" that are localized within the plasma membrane of the two opposed cells ${ }^{10}$. These early works provided the foundation for understanding gap junctions, but lacked sufficient detail to provide mechanistic explanations for how these channels function in cell-to-cell communication.

It was notable, therefore, when the first $3 D$ structure of a gap junction was determined in 1999 by 2D crystallography ${ }^{11}$. This structure was of a connexin isoform called connexin-43 (cx43), an important isoform for cardiac function, and was solved in a lipid bilayer. With an $8 \AA$ resolution in the $x-y$ plane and a $21 \AA$ resolution in the plane of the membrane - a limitation of 2D crystallography - the authors could not build an atomic model into their density map. However, this map did provide information about the dimensions and topology of the channel, as well as the number of alpha helices present in a single gap junction. Figure 1A illustrates the level of detail present in this alpha carbon model. Following this, gap junctions continued to resist high-resolution analysis for another ten years.

In 2009 , the first high-resolution structure of a gap junction was determined by $\mathrm{x}$ ray crystallography ${ }^{12}$. The isoform in this case was connexin-26 (cx26), and the map had sufficient resolution to allow construction of the first atomic model of a 


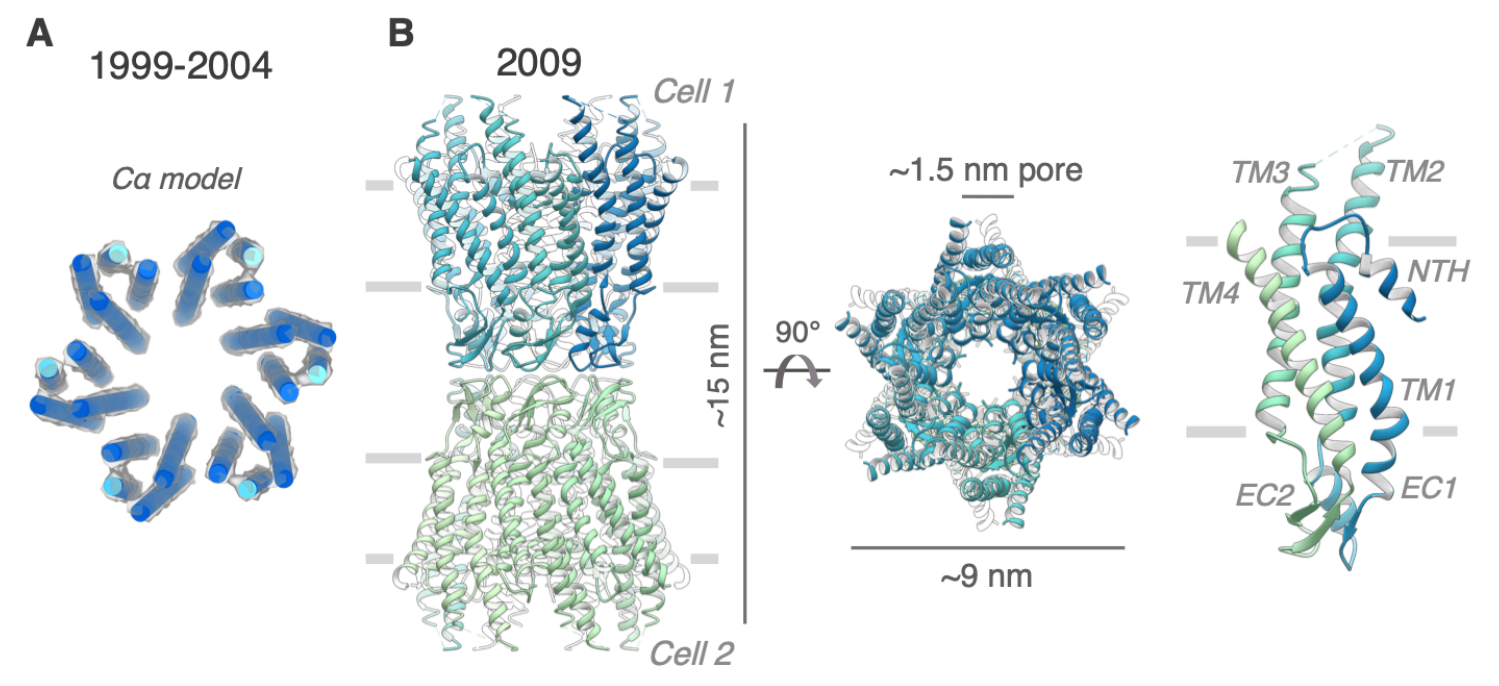

Figure 1. The level of detail in gap junctions models has increased over time. In the 2000's, the gap junctions were limited to alpha carbon models; the model displayed here was built by Yeager, et al (panel A, PDBID 1TXH). In 2009, an atomic model of connexin-26 was built by Maeda, et al (Panel B, $P D B I D$ 2ZW3). The gap junction is composed of 12 connexin subunits, which form a $1.5 \mathrm{~nm}$ pore connecting two neighboring cells.

gap junction intercellular channel. This model provided much more detail than the previous 2D crystallography structure (Figure 1B) and resolved many outstanding questions. The structure revealed in detail the arrangement of the twelve subunits composing the channel. Six connexin subunits form a hemichannel in one cell membrane, which then docks with a hemi-channel in a neighboring cell to form a pore that directly couples the two cells. Each connexin subunit has four transmembrane alpha helices, two extracellular loops responsible for docking interactions between hemichannels - and an n-terminal gating and regulatory domain that folds into the vestibule (e.g., the mouth of the channel) at both ends of the channel (Figure 1B, NTH). 
Connexins also have a cytoplasmic loop region and c-terminal regulatory domain, neither of which were visualized in the connexin-26 x-ray structure, presumably due to their flexibility ${ }^{12}$. This structure defined the connexin fold and the provided a detailed topology of the gap junction pore, and has served as the archetype for gap junction structure-function studies for the last decade.

\section{Structure and Function of Gap Junctions}

Well-regulated cell-to-cell communication is essential for multicellular organisms. For example, electrical signals in the heart and brain are conducted through and coordinated by gap junctions. They also play an important role in maintaining homeostasis across most tissues in the human body by permitting cell-to-cell passage of a wide variety of cellular messengers ${ }^{1}$. There are 21 connexin isoforms in humans - expressed in a cell-type specific manner - and each isoform has unique permeation properties and modes of regulation. Adding to this complexity, most tissues express multiple connexins isoforms, which have the ability to co-assemble. This is thought to provide a mechanism for cells to fine-tune the coupling properties to the local cellular environment ${ }^{8}$.

Gap junctions directly couple neighboring cells through the formation of an intercellular channel that is $15 \mathrm{~nm}$ in length, with a large, $1.5 \mathrm{~nm}$ pore (Figure 1B) $)^{12}$. This large pore is what allows a variety of solutes up to $1 \mathrm{kDa}$ in size to

pass through these unique channels. This includes not only water and ions, but also nutrients, antioxidants, and small signaling molecules like cAMP and $\mathrm{IP}_{3}$. In 
this way, gap junctions allow the passage of chemical information from one cell to the next - as well as across entire tissues and organs. However, the pore size is small enough to impede the passage of larger cellular components like DNA and protein between cells. Although the gap junctions were once thought to be passive pores, they are now recognized as dynamic systems capable of dynamic regulation and gating (i.e., closure of the channel) by various physiological signals (ie. voltage, $\mathrm{pH}, \mathrm{Ca}^{2+}$ and phosphorylation) ${ }^{13,14}$.

Sequence analysis of gap junctions reveals a high level of conservation over their structured regions, indicating that all connexins share a similar topology to that of $\mathrm{Cx} 26$, and pore mobility assays have indicated that all connexins establish large pore channels $(\sim 1.0-1.5 \mathrm{~nm} \text { in diameter depending on the isoform })^{15-17}$. However, despite these structural similarities, different connexin isoforms have different permeability to ions and small molecules, as well as different responsiveness to regulatory signals ${ }^{18}$. For example, channels composed of connexin-43 (cx43) pass ATP more effectively relative to connexin-32 (cx32), but cx32 passes adenosine more efficiently than $c x 43^{19}$. Different isoforms also have different permeability to a variety of dyes and tracers below the size cutoff of the channel - for example, cx32 is less permeable to propidium iodide than cx43 ${ }^{20}$. The divergent properties of connexin isoforms likely reflect the distinct needs of different cell types and tissues, consistent with their cell-type specific expression patterns, but it has been difficult to predict gap junction permeability to complex 
molecules (e.g., signaling molecules) based on their electrical properties obtained by electrophysiology studies ${ }^{21}$.

Further complicating matters, more than one connexin isoform can be coassembled into a single gap junction channel (Figure 2). Hemi-channels may be composed of the same connexin (called a homomeric assembly) or two different connexin isoforms (called a heteromeric assembly) ${ }^{22}$. Hemi-channels can then dock with another channel composed of the same connexin isoform (called a homotypic channel) or of a different connexin isoform (called a heterotypic channel). Heteromeric and heterotypic assembly has been shown to occur in keratinocytes $^{23}$, vascular tissues in the heart ${ }^{24}$, neurons ${ }^{25}$ and the eye lens ${ }^{26,27}$.

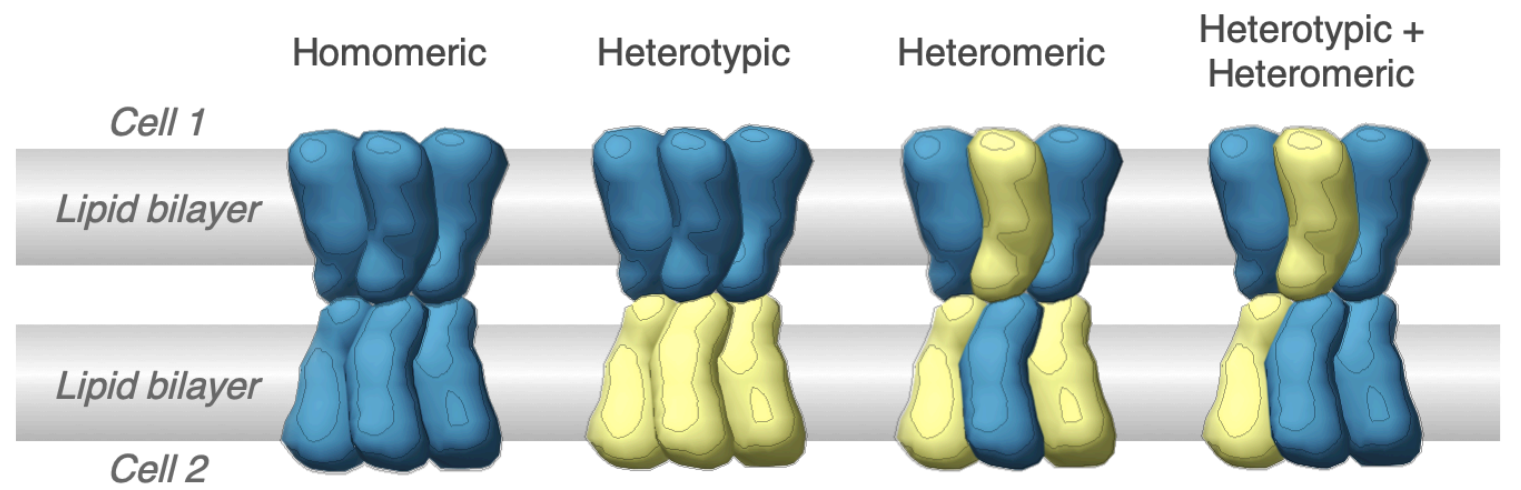

Figure 2. Co-assembly of connexin isoforms can produce a variety of mixed channels. Two different isoforms docking with each other form a heterotypic channel; two isoforms co-assembling in the same hemi-channel form a heteromeric channel.

These different types of assembly are thought to allow the cell to fine tune the properties of the gap junction channel ${ }^{28}$. Isoform specificity observed for heterotypic (docking) interactions are thought to provide a mechanism to allow 
control over cell-type specific communication, i.e., dictating who can talk to whom ${ }^{29}$. For example, in the brain, different types of neurons express different types of connexins, some of which can dock with each other and some of which $\operatorname{cannot}^{30}$. This mechanism appears to direct the formation of information pathways in the brain, as only cells with compatible connexins can effectively communicate via gap junction pathways. The formation of a heterotypic gap junction also results in an asymmetric channel, which imparts directionality on the flow of solutes through the channel (a phenomena known as rectification). Both forms of co-assembly may allow further tailoring of gap junction properties by forming mixed channels with different properties than the parent isoforms alone, but this has yet to be fully understood at a mechanistic level of detail. Notably, there is yet to be any type of discreet pattern of connexin isoform heteromeric assembly - if discrete patterns even exist in cells is unclear. If there is a pattern, this could be important information for understanding the roles of heteromeric and heterotypic channels in regulation.

Gap junctions are not only regulated by changing their composition: they can also open or close (i.e., gate) in response to physiological stimuli ${ }^{1}$. One gating stimulus for gap junctions is voltage changes across the intercellular channel. This trans-junctional voltage response is especially important in electrically coupled cells in the heart and the brain - for example, during action potential propagation $^{31}$. Voltage gating signals are universal amongst the gap junctions all connexin isoforms respond to voltage in some way - but the properties of 
gating are different for different connexin isoforms ${ }^{14}$. A wealth of information about gap junction voltage gating has been uncovered by electrophysiology studies. These functional studies have demonstrated that the n-terminal helix of the connexin is involved in the gating mechanism ${ }^{32}$. The available structural information on the closed gap junction is severely limited. The cx26 crystal structure discussed earlier was apparently isolated in the open state conformation based on the positioning of the n-terminal domain and the crystallization conditions used. A structure of a cx26 disease-state mutant in putative closed form was determined by electron diffraction at a resolution of 7 $\AA^{33}$. The map showed density in the vestibule, suggesting a physical block formed by the $n$-terminal helix, but the low resolution of this map lacked the detail necessary for a mechanistic understanding of voltage gating. Also, while this state is proposed to mimic the voltage-induced state, direct evidence is still lacking.

In addition to voltage, gap junctions can be closed in response to low $\mathrm{pH}$. Low pH is a universal signal of cell stress - for example, during ischemic conditions after heart attack or stroke. Gap junction gating in response to low $\mathrm{pH}$ is proposed to be an important mechanism to stop the spread of cell-stress signals to healthy neighboring cells $-\mathrm{ie}$, apoptotic signals ${ }^{34}$. Current gap junction $\mathrm{pH}$ gating models are based on electrophysiology studies ${ }^{32,35,36}$. From these studies, it has been proposed that $\mathrm{pH}$ gating involves the c-terminal domain of connexin ${ }^{13,14,37}$. Recall that this domain was unresolved in the cx26 crystal structure, presumably due to 
its flexibility ${ }^{12}$. Alternatively, the $n$-terminal helix may also be involved in $\mathrm{pH}$ gating: for Cx50, mutating a single residue in the NTH decreased the sensitivity of $\mathrm{pH}$ gating ${ }^{38}$. Generally, the mechanism of $\mathrm{pH}$ gating lacks detail.

Gap junctions are also gated by calcium ions, a ubiquitous player in cell signaling cascades, often mediated through the action of calmodulin (CaM $)^{39}$. Calcium is also released from the sarcoplasmic reticulum during ischemia after tissue damage in the heart ${ }^{40}$. It is thought that calcium could interact with gap junctions through the binding of $\mathrm{CaM}^{41}$, or through direct binding, which was demonstrated in a recent crystallographic structure of $\mathrm{cx} 26^{42}$. Based on this $\mathrm{Ca}^{2+}$-bound cx26 structure, calcium gating has been proposed to influence channel permeability through an electrostatic mechanism. Calcium also regulates gap junction hemichannels and is proposed to bind to the extracellular loops ${ }^{43}$. This is proposed to keep the undocked hemi-channel closed under normal physiological conditions (i.e., high external concentrations of $\mathrm{Ca}^{2+}$ ) - an important mechanism to prevent cytoplasmic leak. Study of these regulatory mechanisms has been difficult, from a structural perspective, since only the connexin-26 isoform has been determined to a high resolution and flexible regulatory domains such as the c-terminus remain unresolved.

\section{The lens as a model system to study gap junction regulation}

The eye lens provides a model system for studying gap junction regulation. The lens has a highly specialized role in the body - transmitting and focusing light on 
to the retina to support vision - and has developed unique cellular adaptations as a result of its need to be completely transparent ${ }^{44}$. The lens is composed of just two types of cells. A single layer of epithelial cells surrounds the anterior surface, while the rest of the lens mass is composed of specialized cells called fiber cells $^{45}$ (Figure 3). As the lens develops, epithelial cells on the anterior surface of the lens differentiate and elongate into so-called fiber cells. These fiber cells express connexin-46 (cx46) and connexin-50 (cx50), which have been shown biochemically to form heteromeric and heterotypic assemblies in mammalian lens $^{26}$.

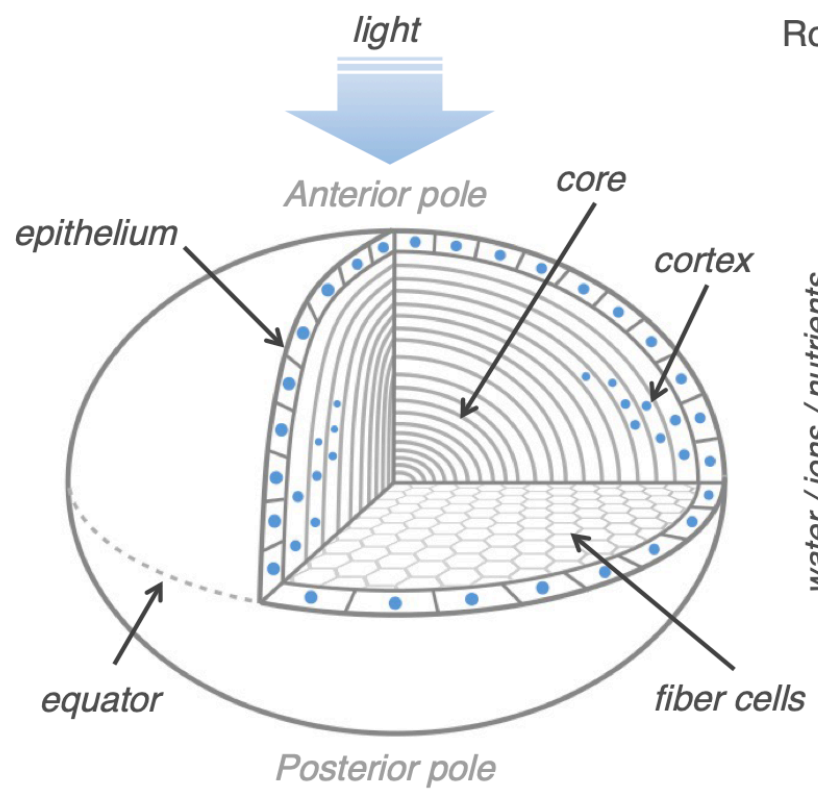

Role of gap junctions in

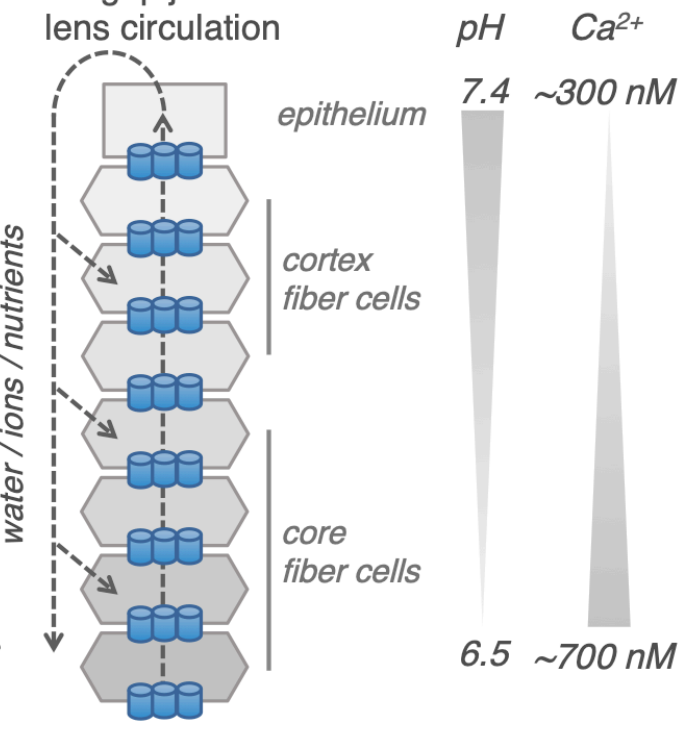

Figure 3. Gap junctions play a key role in the lens circulation system. The lens is formed by the continuous layering of elongated fiber cells. Mature fiber cells shed their organelles to reduce light scattering in the lens, which also lacks vasculature. The lens evolved a microcirculatory system to deliver nutrients to the core and return waste to the center; a network of gap junctions couples this system. The core of the lens presents a unique environment for gap junctions, with a lowered $\mathrm{pH}$ and increased calcium concentration relative to the cortex. 
The bulk of lens tissue is composed of these elongated fiber cells which never turn-over and continuously layer on top of each other - meaning the oldest fiber cells at the core of the lens are as old as the organism ${ }^{46}$. The outermost fiber cells, extending $\sim 15 \%$ depth into the lens, are called differentiating fiber cells (or cortical fiber cells). After differentiation is complete, mature fibers lose of their organelles, including their nuclei, in order to maintain the transparency of the lens ${ }^{47}$. This means these fiber cells must survive for the life of organism without new protein synthesis.

As a result of the anaerobic respiration that occurs in the mature fiber cells, the $\mathrm{pH}$ in the lens drops from about 7.4 in the epithelial cells to about 6.5 in the core of the lens ${ }^{48}$. In addition, calcium concentration increases from $\sim 300 \mathrm{nM}$ to $\sim 700$ $\mathrm{nM}$ in the core ${ }^{49}$, meaning that proteins in the mature fibers face a distinctively different environment than those in the surface fiber cells. As such, one key feature of mature fiber cells is the modification of gap junctions. In mature fibers, the c-terminal domain is cleaved from both connexin- 46 and -50 , the intercellular loop region is also cleaved; both connexins become hyper-phosphorylated ${ }^{22,50,51}$. It has been proposed that these modifications help keep these gap junctions functional in the acidic core of the lens ${ }^{52}$.

In order to accomplish its role of focusing light on the retina, the lens must be transparent and therefore lacks vasculature. Therefore, the lens has evolved a specialized micro-circulation system in which gap junctions play a key role ${ }^{46}$. This 
circulation system delivers nutrients to the core of the organ and removes the waste produced by the anaerobic metabolism of the fiber cells ${ }^{46}$. An Na/KATPase expressed only in the lens epithelial cells establishes an ionic gradient that drives this circulation system. These pumps establish a sodium gradient, with higher sodium concentration in the extracellular spaces in the outer region of the lens and lower sodium concentrations in the center of the lens. This difference in sodium concentration drives water flow down the sodium gradient into the core of the lens, which also brings other solutes to the core (Figure 3, arrows). Sodium and water flow back out of the core of the lens through a lowresistance network of gap junctions that couple fiber cells - carrying along metabolic waste products as they go. In this way, the lens gap junctions play a critical role in maintaining homeostasis of the lens and transparency throughout our lifetimes. This essential role is supported by genetic mutations that disrupt these proteins structure and/or function result in cataract formation during early childhood $^{49,53}$. 


\section{References}

1. Goodenough, D. A. \& Paul, D. L. Gap Junctions. Cold Spring Harb. Perspect. Biol. 1, a002576-a002576 (2009).

2. White, T. W., Deans, M. R., Kelsell, D. P. \& Paul, D. L. Connexin mutations in deafness. Nature 394, 630-1 (1998).

3. Bergoffen, J. et al. Connexin mutations in X-linked Charcot-Marie-Tooth disease. Science (80-. ). (1993). doi:10.1126/science.8266101

4. Richard, G. Connexin disorders of the skin. Clinics in Dermatology (2005). doi:10.1016/j.clindermatol.2004.09.010

5. White, T. W. Unique and redundant connexin contributions to lens development. Science 295, 319-20 (2002).

6. Paznekas, W. A. et al. GJA1 mutations, variants, and connexin 43 dysfunction as it relates to the oculodentodigital dysplasia phenotype. Human Mutation (2009). doi:10.1002/humu.20958

7. Nielsen, M. S. et al. Gap junctions. Compr. Physiol. (2012). doi:10.1002/cphy.c110051

8. Kumar, N. M. \& Gilula, N. B. The gap junction communication channel. Cell 84, 381-388 (1996).

9. $\quad$ Caspar, D. L. D., Goodenough, D. A., Makowski, L. \& Phillips, W. C. Gap junction structures. I. Correlated electron microscopy and X-ray diffraction. J. Cell Biol. (1977). doi:10.1083/jcb.74.2.605

10. Unwin, P. N. T. \& Ennis, P. D. Two configurations of a channel-forming membrane protein. Nature 307, 609-613 (1984). 
11. Unger, V. M., Kumar, N. M., Gilula, N. B. \& Yeager, M. Expression, twodimensional crystallization, and electron cryo-crystallography of recombinant gap junction membrane channels. J. Struct. Biol. 128, 98-105 (1999).

12. Maeda, S. et al. Structure of the connexin 26 gap junction channel at $3.5 \mathrm{~A}$ resolution. Nature 458, 597-602 (2009).

13. Peracchia, C. Chemical gating of gap junction channels: Roles of calcium, $\mathrm{pH}$ and calmodulin. Biochimica et Biophysica Acta - Biomembranes 1662, $61-80(2004)$.

14. Bukauskas, F. F. \& Verselis, V. K. Gap junction channel gating. Biochimica et Biophysica Acta - Biomembranes 1662, 42-60 (2004).

15. Beblo, D. a. \& Veenstra, R. D. Monovalent Cation Permeation through the Connexin40 Gap Junction Channel. J. Gen. Physiol. 109, 509-522 (1997).

16. Wang, H.-Z. \& Veenstra, R. D. Monovalent Ion Selectivity Sequences of the Rat Connexin43 Gap Junction Channel. J. Gen. Physiol. 109, 491-507 (1997).

17. Gong, X.-Q. \& Nicholson, B. J. Size Selectivity Between Gap Junction Channels Composed of Different Connexins. Cell Commun. Adhes. 8, 187-192 (2008).

18. Goldberg, G. S., Valiunas, V. \& Brink, P. R. Selective permeability of gap junction channels. Biochimica et Biophysica Acta - Biomembranes 1662, 96-101 (2004). 
19. Goldberg, G. S., Lampe, P. D. \& Nicholson, B. J. Selective transfer of endogenous metabolites through gap junctions composed of different connexins. Nat. Cell Biol. 1, 457-459 (1999).

20. C. Elfgang H. Lichtenberg-Frate, A. Butterweck, O. Traub, R. A. Klein, D. F. Hulser, and K. Willeck, R. E. Specific permeability and selective formation of gap junction channels in connexin-transfected HeLa cells. J. Cell Biol. 129, 805-817 (1995).

21. Veenstra, R. D. et al. Selectivity of connexin-specific gap junctions does not correlate with channel conductance. Circ. Res. (1995). doi:10.1161/01.RES.77.6.1156

22. Mathias, R. T., White, T. W. \& Gong, X. Lens Gap Junctions in Growth, Differentiation, and Homeostasis. Physiol. Rev. 90, 179-206 (2010).

23. Brissette, J. L., Kumar, N. M., Gilula, N. B., Hall, J. E. \& Dotto, G. P. Switch in gap junction protein expression is associated with selective changes in junctional permeability during keratinocyte differentiation. Proc. Natl. Acad.

Sci. U. S. A. 91, 6453-7 (1994).

24. Haefliger, J. A., Nicod, P. \& Meda, P. Contribution of connexins to the function of the vascular wall. Cardiovasc. Res. 62, 345-356 (2004).

25. Bennett, M. V. L. \& Zukin, R. S. Electrical Coupling and Neuronal Synchronization in the Mammalian Brain. Neuron 41, 495-511 (2004).

26. Jiang, J. X. \& Goodenough, D. A. Heteromeric connexons in lens gap junction channels. Proc. Natl. Acad. Sci. U. S. A. 93, 1287-91 (1996). 
27. Vaney, D. I. \& Weiler, R. Gap junctions in the eye: Evidence for heteromeric, heterotypic and mixed-homotypic interactions. Brain Research Reviews 32, 115-120 (2000).

28. White, T. W. \& Bruzzone, R. Multiple Connexin Proteins in Single Intercellular Channels: Connexin Compatibility and Functional Consequences. J. Bioenerg. Biomembr. 28, 339-350 (1996).

29. Koval, M., Molina, S. A. \& Burt, J. M. Mix and match: Investigating heteromeric and heterotypic gap junction channels in model systems and native tissues. in FEBS Letters (2014). doi:10.1016/j.febslet.2014.02.025

30. Rash, J. E., Yasumura, T., Dudek, F. E. \& Nagy, J. I. Cell-specific expression of connexins and evidence of restricted gap junctional coupling between glial cells and between neurons. J. Neurosci. (2001).

31. Miller, A. C. \& Pereda, A. E. The electrical synapse: Molecular complexities at the gap and beyond. Dev. Neurobiol. 77, 562-574 (2017).

32. Kronengold, J., Srinivas, M. \& Verselis, V. K. The N-terminal half of the connexin protein contains the core elements of the pore and voltage gates. J. Membr. Biol. 245, 453-463 (2012).

33. Oshima, A., Tani, K., Hiroaki, Y., Fujiyoshi, Y. \& Sosinsky, G. E. Threedimensional structure of a human connexin26 gap junction channel reveals a plug in the vestibule. Proc. Natl. Acad. Sci. 104, 10034-10039 (2007).

34. Andrade-Rozental, A. F. et al. Gap junctions: The 'kiss of death' and the 'kiss of life'. Brain Research Reviews 32, 308-315 (2000). 
35. Anumonwo, J. M. et al. The carboxyl terminal domain regulates the unitary conductance and voltage dependence of connexin40 gap junction channels. Circ. Res. 88, 666-673 (2001).

36. Trexler, E. B., Bennett, M. V, Bargiello, T. a \& Verselis, V. K. Voltage gating and permeation in a gap junction hemichannel. Proc. Natl. Acad. Sci. U. S. A. 93, 5836-5841 (1996).

37. Bennett, B. C. et al. An electrostatic mechanism for $\mathrm{Ca}(2+)$-mediated regulation of gap junction channels. Nat Commun 7, 8770 (2016).

38. Peracchia, C. \& Peracchia, L. L. Inversion of both gating polarity and CO2 sensitivity of voltage gating with D3N mutation of Cx50. Am. J. Physiol. Cell Physiol. 288, C1381-C1389 (2005).

39. KLEVIT, R. E., DALGARNO, D. C., LEVINE, B. A. \& WILLIAMS, R. J. P. 1H-NMR studies of calmodulin: The nature of the Ca2+-dependent conformational change. Eur. J. Biochem. (1984). doi:10.1111/j.14321033.1984.tb07983.x

40. Carmeliet, E. Cardiac Ionic Currents and Acute Ischemia: From Channels to Arrhythmias. Physiol. Rev. (2017). doi:10.1152/physrev.1999.79.3.917

41. Zou, J. et al. Gap junction regulation by calmodulin. in FEBS Letters 588, 1430-1438 (Federation of European Biochemical Societies, 2014).

42. Bennett, B. C. et al. An electrostatic mechanism for Ca 2+-mediated regulation of gap junction channels. Nat. Commun. 7, 8770 (2016). 
43. Gomez-Hernandez, J. M., de Miguel, M., Larrosa, B., Gonzalez, D. \& Barrio, L. C. Molecular basis of calcium regulation in connexin-32 hemichannels. Proc. Natl. Acad. Sci. (2003).

doi:10.1073/pnas. 2530348100

44. Mathias, R. T., Kistler, J. \& Donaldson, P. The Lens Circulation. J. Membr. Biol. 216, 1-16 (2007).

45. Mathias, R. T., Rae, J. L. \& Baldo, G. J. Physiological properties of the normal lens. Physiol Rev 77, 21-50 (1997).

46. Mathias, R. T., Kistler, J. \& Donaldson, P. The lens circulation. Journal of Membrane Biology 216, 1-16 (2007).

47. Bassnett, S. Lens Organelle Degradation. Exp. Eye Res. (2002). doi:10.1006/exer.2001.1111

48. Mathias, R. T., Riquelme, G. \& Rae, J. L. Cell to cell communication and pH in the frog lens. J. Gen. Physiol. (1991).

49. Gao, J. et al. Connections Between Connexins, Calcium, and Cataracts in the Lens. J. Gen. Physiol. (2004). doi:10.1085/jgp.200409121

50. Wang, Z. \& Schey, K. L. Phosphorylation and truncation sites of bovine lens connexin 46 and connexin 50. Exp. Eye Res. (2009). doi:10.1016/j.exer.2009.07.015

51. Shearer, D., Ens, W., Standing, K. \& Valdimarsson, G. Posttranslational modifications in lens fiber connexins identified by off-line-HPLC MALDIquadrupole time-of-flight mass spectrometry. Invest Ophthalmol Vis Sci 49, 1553-1562 (2008). 
52. Sheng Lin, J., Eckert, R., Kistler, J. \& Donaldson, P. Spatial differences in gap junction gating in the lens are a consequence of connexin cleavage. Eur. J. Cell Biol. (2012). doi:10.1016/s0171-9335(98)80002-2

53. DeRosa, A. M., Xia, C.-H., Gong, X. \& White, T. W. The cataract-inducing S50P mutation in Cx50 dominantly alters the channel gating of wild-type lens connexins. J. Cell Sci. 120, 4107-16 (2007). 


\section{Chapter 3}

\section{Structural Characterization of Native Lens Connexin-46/50 Gap Junction Channels in the open state by CryoEM}

\section{This chapter has been published}

Structure of native lens connexin-46/50 intercellular channels by CryoEM. Nature 564, 372-377 (2018). Janette B. Myers ${ }^{1, \dagger}$, Bassam G. Haddad ${ }^{1, \dagger}$, Susan E. O'Neill', Dror S. Chorev ${ }^{3}$, Craig C. Yoshioka ${ }^{2}$, Carol V. Robinson ${ }^{3}$, Daniel M. Zuckerman $^{2}$ and Steve L. Reichow ${ }^{1, *}$

${ }^{1}$ Department of Chemistry, Portland State University, Portland OR 97201, U.S.A. 2 Department of Biomedical Engineering, Oregon Health and Sciences University, Portland OR 97239, U.S.A.

${ }^{3}$ Physical and Theoretical Chemistry Laboratory, University of Oxford, Oxford OX1 3QZ, U.K.

${ }^{\dagger}$ Indicates equal contribution *Correspondence: reichow@pdx.edu (S.L.R.)

This project succeeded due to the contribution of many individuals. Susan O'Neil performed the biochemical isolation of gap junctions. Janette Myers prepared and screened cryoEM grids. With the help fo Craig Yoshioka, Janette Myers collected electron microscopy data, and JM processed the resulting data and built atomic models. Bassam Haddad performed molecular dynamics simulations with the guidance of Daniel Zuckerman. Dror Chrorev of Carol Robinson's lab performed mass spectroscopy on native gap junctions provided by our lab. Steve Reichow directed the project. 


\section{Abstract}

Gap junctions establish direct pathways for cell-to-cell communication, through the assembly of twelve subunits (connexins) that form intercellular channels connecting neighboring cells. Co-assembly of different connexin isoforms produces channels with unique properties, and enables communication across cell-types. To gain access into the structural underpinnings of connexin coassembly, we used single particle CryoEM to determine the structure of native lens gap junction channels, composed of connexin-46 and connexin-50 (Cx46/50). We provide the first comparative analysis to connexin-26 (Cx26), which together with computational studies elucidates key energetic features governing gap junction perm-selectivity. Cx46/50 adopts an open-state conformation that is unique from the Cx26 crystal structure, yet appears to be stabilized by a conserved set of hydrophobic anchoring residues. 'Hot spots' of genetic mutations linked to hereditary cataract formation map to the core structural-functional elements identified in $\mathrm{Cx} 46 / 50$, rationalizing many of the disease-causing effects.

\section{Main Text}

Cell-to-cell communication directed by the gap junctions is essential to neuronal function, cardiac coupling, and for coordinating intercellular signaling and metabolic activity in most tissues (e.g. heart, skin, liver and eye lens) ${ }^{1}$. As such, genetic mutation or aberrant regulation is linked to a variety of pathological 
conditions, including cardiac arrhythmia, stroke, blindness, deafness, skin disease and cancers ${ }^{2-4}$.

Intercellular channel formation is achieved through an assembly of twelve subunits, known as connexins ${ }^{5}$. Within the plasma membrane, six connexins are organized into a hemi-channel structure. Hemi-channels from neighboring cells dock together to form complete cell-to-cell channels, which cluster to form large gap junction plaques. A remarkably large channel pore provides passage to an array of chemical information; including ions, metabolites, hormones, and other small signaling molecules less than $\sim 1 \mathrm{kDa}$ in size (e.g., $\mathrm{K}^{+}$, cyclic-AMP (cAMP), inositol triphosphate $\left(\mathrm{IP}_{3}\right)$ and glucose). In this way, interconnected cells may exchange electrical and chemical information across an entire tissue or organ.

Humans express 21 connexin isoforms in a cell-type specific fashion ${ }^{6}$. Most cells express multiple isoforms and certain connexins display an ability to coassemble, either by docking two hemi-channels composed of different isoforms (heterotypic), or through mixed isoform assembly within the same hemi-channel (heteromeric). This complexity is thought to allow cells to fine-tune the conductance of chemical information, and support coupling across different cell types ${ }^{7}$. However, our understanding of the physical basis for connexin isoform compatibility, conductance, substrate selectivity and channel gating remains limited $^{8,9}$, as high-resolution structural information obtained by crystallographic 
analysis has so far been restricted to just a single model system, connexin-26 $(\mathrm{C} \times 26)^{10,11}$.

To gain further insight into the mechanistic effects of gap junction isoform diversity and heteromeric assembly, we applied single particle imaging methods by electron cryo-microscopy (CryoEM) to elucidate the structure of native connexin-46 and $-50(\mathrm{Cx} 46 / 50)$ channels, isolated from the eye lens, where connexin-mediated communication is required for growth, differentiation and maintenance of lens transparency required to support vision ${ }^{12}$. Comparative molecular dynamics (MD) simulations reveal key features of ion permeation and selectivity, and suggest Cx46/50 adopts a more stable open-state conformation compared to the previously described Cx26 crystal structure ${ }^{10}$.

\section{Structural overview of $\mathrm{Cx} 46 / 50$}

Cx46/50 form heteromeric/heterotypic intercellular channels in the mammalian lens ${ }^{13,14}$. We isolated native $\mathrm{C} \times 46 / 50$ intercellular channels from core lens tissue (sheep Cx44/49), and verified heteromeric co-assembly by biochemical analysis and chemical cross-linking mass-spectrometry (MS) (Extended Data Fig. 1). The structure of $\mathrm{Cx} 46 / 50$ intercellular channels was resolved by single particle CryoEM to near-atomic resolution (3.4 and $3.5 \AA$ Å) (Fig. 1a-b, Extended Data Figs. 1-3). The resulting density maps revealed a $15 \mathrm{~nm}$ tall dodecameric (12mer) channel with a girdled waist ( 6-9 nm wide). When rotated $90^{\circ}$, a large 

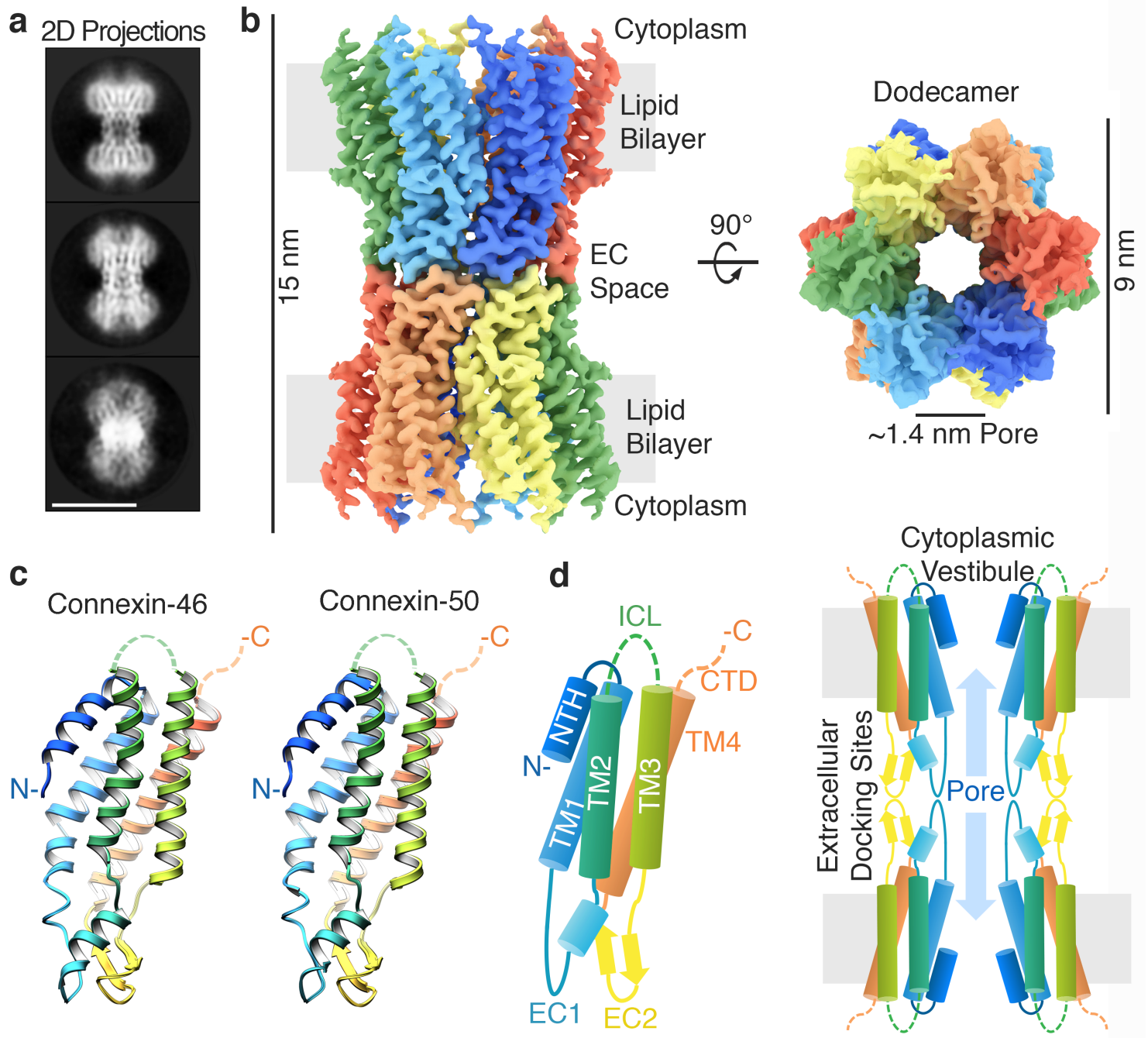

Figure 1. Structure of connexin-46/50 intercellular channels. (a) Representative projection averages of native Cx46/50 intercellular channels (selected from 165 classes). Scale bar $=10 \mathrm{~nm}$. (b) 3D CryoEM reconstruction displaying the twelve subunits colored independently. Regions corresponding to the lipid bilayer, cytoplasm and extracellular (EC) space are indicated. (c) Atomic models of Cx46 (left) and Cx50 (right) monomers displayed in ribbon representation. (d) (left) Diagram of the connexin-fold and (right) cross-section of the assembled intercellular channel, with domains labeled: transmembrane domains 1-4 (TM1-4), extracellular domains 1-2 (EC1-2), and n-terminal helix domain (NTH). The intracellular loop (ICL) and c-terminal domains (CTD) were not visualized in the CryoEM density (dotted lines). EC1/2 form docking sites, establishing a continuous channel pore of $\sim 1.4 \mathrm{~nm}$ diameter connecting the cytoplasm of neighboring cells. 
unobstructed pore of $\sim 1.4 \mathrm{~nm}$ diameter is visualized along the channel axis, consistent with the proposed open-state conformation (Fig. 1b).

Despite significant effort, we were unable to resolve a specific pattern of $\mathrm{Cx} 46 / 50$ heteromeric or heterotypic co-assembly using 3D-classification/refinement strategies (Methods and Extended Data Fig. 4,5). Nevertheless, high-resolution features corresponding to sidechain densities are observed throughout the reconstructions following 12 -fold symmetry refinement (i.e., by averaging signal contributed by both $\mathrm{Cx} 46$ and $\mathrm{Cx} 50$ ). Thus, these two isoforms, which share $\sim 80 \%$ core sequence identity ( $88 \%$ similarity), also share a highly similar $3 \mathrm{D}$ structure (Fig 1b-c, Extended Data Fig. 4-6), consistent with the ability of Cx46/50 to co-assemble in a variety of heteromeric/heterotypic states.

Atomic models for Cx46 and Cx50 were built into the averaged CryoEM density map, and various heteromeric/heterotypic channels were constructed for comparative analysis. While the structures display excellent validation statistics (Extended Data Fig. 2), local resolution assessment between the atomic models and experimental density identified features of both models that were less well defined by the density map, in particular at sites where the two isoforms differ in sequence (Extended Data Fig. 3-5 and Supplementary Table 2,3). We note, analysis of the models presented should be approached with caution due to intrinsic limitations of our heterogeneous dataset, which may go beyond local 
differences in primary sequence; for example, due to the possibility of one isoform being more well-ordered and potentially biasing interpretation.

The refined $C x 46 / 50$ structures comprise four alternating transmembrane $\alpha$ helices (TM1-4), two extracellular domains (EC1/2) connecting TM1/2 and TM3/4 respectively, and an n-terminal helix (NTH) domain that folds into the channel vestibule and is connected to the pore-lining TM1 helix via a short linker (Fig. 1c,d). Density for Met1 is not observed in the CryoEM maps and was determined to be removed in both $\mathrm{Cx} 46$ and $\mathrm{Cx} 50$ by MS/MS, and the resulting n-terminal glycine (G2) is partially acetylated (Extended Data Fig. 1), as shown for the bovine isoforms ${ }^{15,16}$. The intracellular loop (ICL) connecting TM2/3 and cytoplasmic c-terminal domain (CTD) harboring the native cleavage sites of Cx46 and Cx50 are also not resolved ${ }^{16}$. The ICL and CTD were not observed in the crystallographic structures of $\mathrm{Cx} 26$ either ${ }^{10,11}$, presumably due to intrinsic disorder in these regulatory domains.

The close structural similarity of Cx46 and Cx50 results in highly similar interfacial interactions that include conserved regions of hydrophobic packing over the TM region and a strikingly similar hydrogen bond/ion-pair network between adjacent (heteromeric interface, Fig. 2a-b, Extended Data Fig. 6) and opposed subunits (heterotypic interface, Fig. 2c-d, Extended Data Fig. 6). Most of these stabilizing interactions are present in $\mathrm{C} \times 26^{10,17}$, including the EC1 Q/N motif (Fig 2c) and EC2 paring involving the K/R-N-D motif (Fig 2d), a conserved 


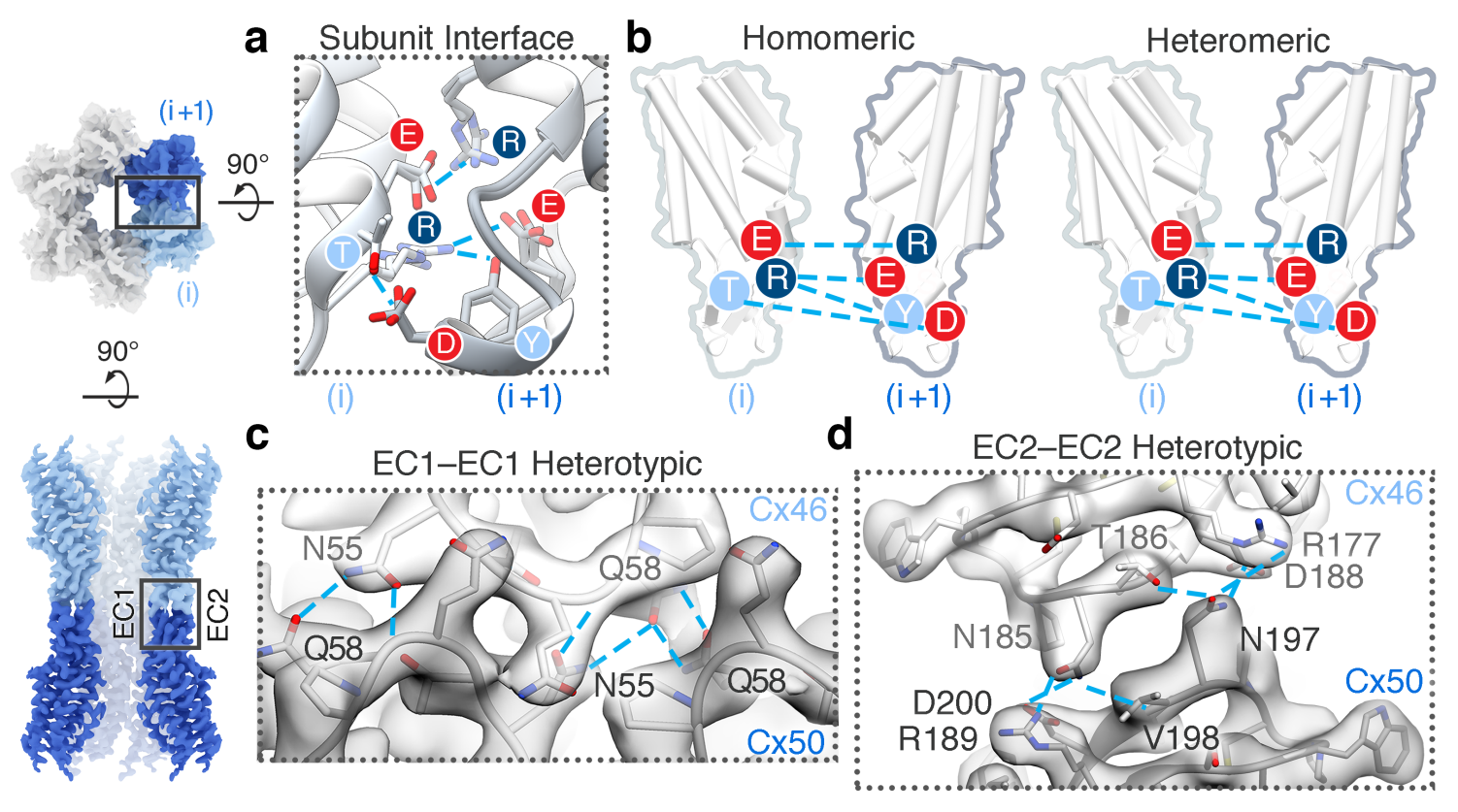

Figure 2. Heteromeric and heterotypic interactions between Cx46 and Cx50. (a) Structural overlay and (b) illustration of the conserved electrostatic network between neighboring subunits ( $i$ and $i+1)$ identified in homomeric Cx46 and Cx50 and heteromeric Cx46/50 models. Labels are colored according to amino acid charge characteristics (red - negative, dark blue positive and light blue - polar). (c) Zoom-view of EC1-EC1 and (d) EC2-EC2 docking site interactions, with atomic models of Cx46 and Cx50 hemi-channels built into the CryoEM density in a heterotypic configuration. Conserved amino acids involved in hydrogen bond pairing (cyan lines) are labeled.

element amongst Group I heterotypic compatible isoforms ${ }^{18}$. Although Cx46/50 and Cx26 are not classified as heteromeric compatible channels ${ }^{19}$, the conserved features at the heteromeric interface are congruent with the current understanding that heteromeric co-assembly of connexins is established during biogenesis in the ER/Golgi network ${ }^{19}$.

Overall, despite significant sequence differences, Cx46/50 ( $\alpha$-family connexins) display core structural features that are very similar to the $\beta$-family member, Cx26 $6^{10}$ (pair-wise $\mathrm{C} \alpha$ root mean squared deviation (r.m.s.d.) $=2.18 \AA$ and $2.14 \AA$ 
versus Cx46 and Cx50, respectively). Thus, these different connexin family members share a conserved connexin-fold and gap junction channel architecture, as presented in Fig $\mathbf{1 d}$, and are consistent with early low-resolution electron diffraction studies on connexin-43 (Cx43) obtained in a lipid bilayer ${ }^{20,21}$. Despite these general similarities; however, we uncovered significant differences between $\mathrm{Cx} 46 / 50$ and $\mathrm{Cx} 26$ localized to key functional sites that are expected to contribute to isoform-specific permeation and selectivity properties and provide insight into the interactions responsible for fully stabilizing the open-state conformation of these channels, detailed below.

\section{Energetics of Ion Permeation/Selectivity}

Comparisons between Cx50, Cx46 and Cx26 intercellular channels reveal unique electrostatic pore pathways, with shared regions of negative charge potential and steric constriction sites (CS) formed by the NTH domains that narrow the cytoplasmic vestibule (CV) to $\sim 10-12 \AA$, at both ends of the channel (Fig. 3a,b). The pore diameters are within the range determined for other connexin channels ${ }^{22}$, and fitting with the general ability of gap junctions to permeate a variety of molecules less than $\sim 1 \mathrm{kDa}$ in size (e.g., Fig 3b, labeled 1-5). Remarkably; however, these channels can display a substantial level of isoformspecific selectivity for molecules beneath this size cut-off, even discriminating between small charged ions ${ }^{23}$. 


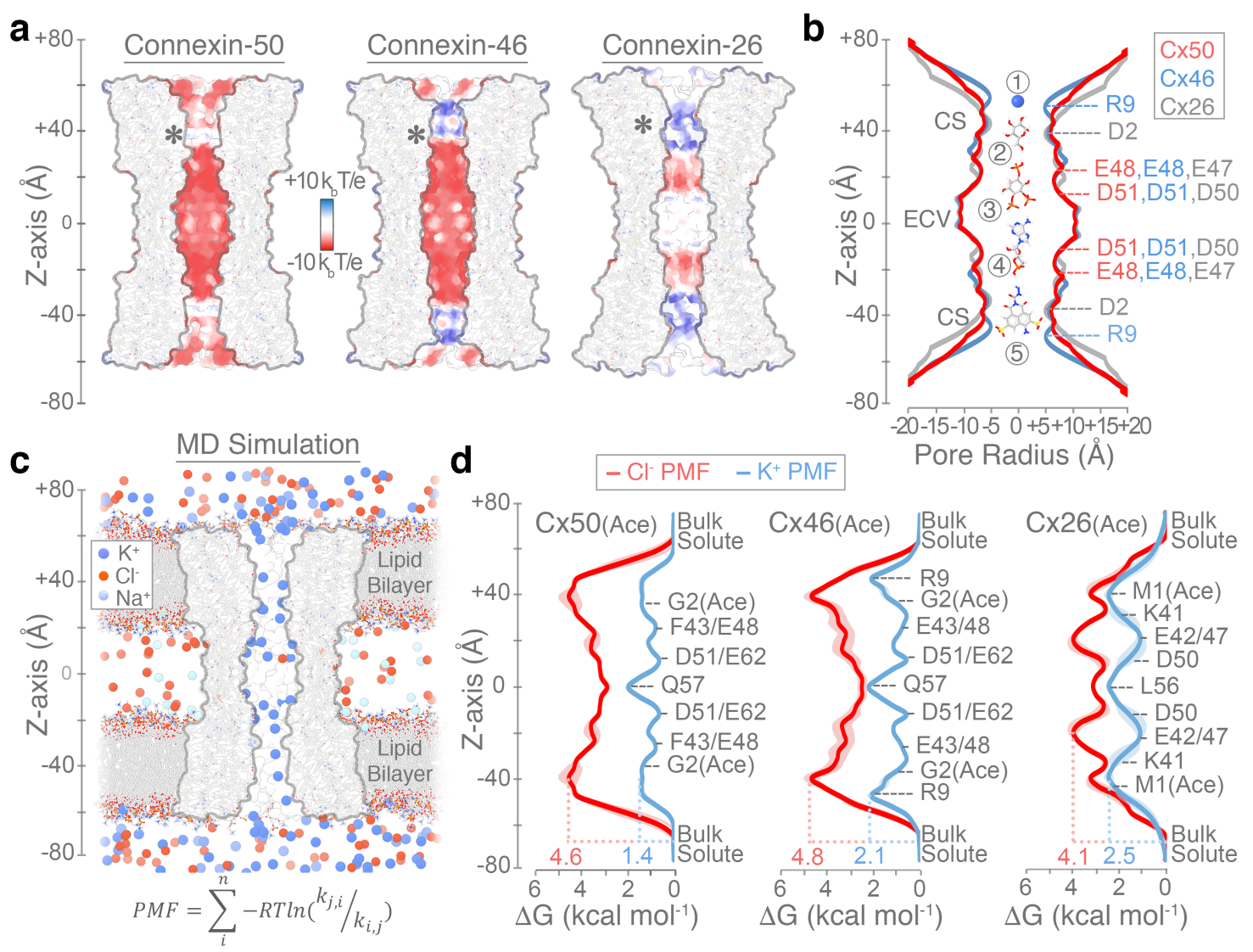

Figure 3. Comparative pore profile and energetics of ion permeation. (a) Cut-away surface representation of Cx50 (left), Cx46 (center) and Cx26 (right) (PDB 2ZW3 ${ }^{10}$; residues 2-96 and 132-217), colored by coulombic potential (negative - red, neutral - white and positive - blue). Asterisk indicates the site of $n$-terminus. (b) Pore radius determined for experimental structures of $C \times 50$ (red), Cx46 (blue) and Cx26 (grey, PDB 2ZW3 ${ }^{10}$ ). Locations of constriction sites (CS) and extracellular vestibule (ECV) are indicated. Structures of representative substrates are displayed to scale $\left(1-K^{+}\right.$ion, $2-$ glucose, $3-$ $I P_{3}, 4-c A M P$ and 5 - Lucifer Yellow) (c) Snapshot of the Cx50 MD simulation, showing the membrane channel (white) embedded in two lipid bilayers and solvated in the presence of intracellular $\mathrm{K}^{+}$, extracellular $\mathrm{Na}^{+}$and $\mathrm{Cl}$ ions. Water molecules not shown. (d) Potential-of-mean-force (PMF) describing the free-energy landscape (DG, $\mathrm{kcal} \mathrm{mol}^{-1}$ ) experienced by $\mathrm{K}^{+}$ions (blue trace) and $\mathrm{Cl}$ ions (red trace) permeating the channel pore.

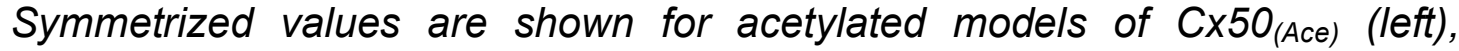
Cx46 (Ace) $($ center) and Cx26(Ace) (right), with non-symmetrized values in lighter shading. Amino acid positions are presented for correlation purposes, and do not represent deconvolution of the free-energy components. 
To validate our structural models and gain insight into the mechanism of ion perm-selectivity, we conducted comparative all-atom molecular dynamics (MD) simulations and potential-of-mean-force (PMF) calculations to define the freeenergy landscape of potassium $\left(\mathrm{K}^{+}\right)$and chloride $\left(\mathrm{Cl}^{-}\right)$permeation for $\mathrm{Cx} 50, \mathrm{Cx} 46$ and Cx26 (Fig. 3c-d, Extended Data Fig. 7,8). PMFs obtained for Cx26 should be interpreted cautiously due to significant dynamical behavior observed for the NTH domain during MD simulations (Extended Data Fig. 7), described in detail in the following section.

Cx50, Cx46 and Cx26 form high conductance ion channels, with preference for conductance of cations over anions. For MD simulation, the n-terminus of each of the models was acetylated $\left(\mathrm{C} \times 50_{(\mathrm{Ace})}, \mathrm{C} \times 46_{(\mathrm{Ace})}\right.$ and $\left.\mathrm{C} \times 26_{(\mathrm{Ace})}\right)$, as this form is expected to represent the predominant species in $v^{2}{ }^{24}{ }^{4}$, and previous MD studies suggest this co-translational modification is required to obtain physiologically relevant charge selectivity of $\mathrm{C} \times 26^{22}$. PMFs for $\mathrm{K}^{+}$, the major permeant ion, reveal peak energetic barriers within the CS regions, ranging from $1.4 \mathrm{kcal} \mathrm{mol}^{-1}$ for $\mathrm{Cx} 50_{\text {(Ace) }}$, to $2.1 \mathrm{kcal} \mathrm{mol}^{-1}$ in $\mathrm{C} \times 46_{(\text {Ace) }}$ and $2.5 \mathrm{kcal} \mathrm{mol}^{-1}$ in Cx26 (Ace) $($ Fig. 3d). These relatively low barriers are similar to the peak energetic barrier determined for other high-conductance $\mathrm{Na}$ - and K-channels $(\sim 2-3 \mathrm{kcal}$ $\left.\mathrm{mol}^{-1}\right)^{25-27}$, and are consistent with the range of experimental unitary conductance values of these channels, with $\mathrm{Cx} 50>\mathrm{C} \times 46 \approx \mathrm{C} \times 26\left(\sim 220 \mathrm{pS}\right.$ for $\mathrm{Cx} 50^{28}$ vs. -140-135 pS for $\mathrm{C} \times 46^{29}$ and $\mathrm{C} \times 26^{30}$; in 130-140 mM CsCl). 
The differences in $\mathrm{K}^{+}$PMF correlate with isoform-specific differences in both steric and electrostatic environments. The CS of $\mathrm{Cx50}$ (Ace) displays the lowest barrier and is characterized by a nearly complete electronegative coulombic potential, due to neutralization of the n-terminus by acetylation (asterisk in Fig. 3a and Extended Data Fig. 8). The major $\mathrm{K}^{+}$energy barrier of $\mathrm{Cx} 46_{(\mathrm{Ace})}$ correlates with the position of positively charged R9 residue ( $50 \AA$ z-axis) (Fig. 3d, Extended Data Fig. 8), which also constricts the CS of Cx46 to $\sim 10 \AA$ in our model (vs. $\sim 12 \AA$ for Cx50) (Fig. 3b). However, the CryoEM density map is not well defined at this site (Supplementary Table 2), likely due (at least in part) to the conformational flexibility of this residue, as dynamical behavior is observed during MD simulation. These dynamics of R9 effectively modulate the steric barrier of Cx46 ( 10-12 $\AA$ ). The CS K $\mathrm{K}^{+}$energy barrier of Cx26 (Ace) Correlates with the location of the basic residue K41 ( $50 \AA$ z-axis) (Fig. 3d), located on TM1 just below the NTH domain, as previously reported ${ }^{22,32}$.

Free energy minima for $\mathrm{K}^{+}$are localized within the extracellular vestibule (ECV; $\sim 10$ and $\sim 30 \AA$, z-axis) of all three isoforms (Fig. 3d), supporting the role of EC1 in establishing charge selectivity and conductance ${ }^{31-33}$. In Cx46 and Cx50, several negatively charged residues (e.g., E48, D51 and E62) localize with regions of high $\mathrm{K}^{+}$ion density (Fig. 3c-d, Extended Data Fig. 9). Notably, charge substitutions at D51 resulted in decreased unitary conductance in Cx46 hemichannels $^{34}$. The residues E48 and D51 are conserved in Cx26 (equivalent to E47 and D50) (Fig. 3d), and establish transient binding interactions with $\mathrm{K}^{+}$ions 
during MD simulation. These sites have also been implicated in $\mathrm{Ca}^{2+}$ regulation in Cx26 by X-ray crystallography ${ }^{11}, \mathrm{MD}$ studies ${ }^{35,36}$, and by functional mutation studies of $\mathrm{C} \times 46^{36}$. Therefore, competitive $\mathrm{K}^{+}$binding at these sites may contribute to the mechanism of $\mathrm{Ca}^{2+}$ regulation/sensitivity. E62 (in Cx46/50) appears to form an additional cation binding site, through coordination by the carboxylate sidechain and nearby backbone carbonyl oxygens (Extended Data Fig. 9). E62 is not conserved in other human connexin isoforms (with the exception of Cx43), and therefore may constitute an isoform-specific regulatory site. Extracellular $\mathrm{Ca}^{2+}$ is involved in the mechanism of closing (or gating) connexin hemichannels ${ }^{37}$, and competition by $\mathrm{K}^{+}$binding at this putative site may contribute to the mechanism of potentiation of $\mathrm{C} \times 50$ and $\mathrm{C} \times 46$ hemi-channels by extracellular $\mathrm{K}^{+}$ions $^{38}$.

Cx50, Cx46 and Cx26 display an appreciable level of selectivity toward positively charged small ions, with permeability ratios of $\mathrm{K}^{+}$to $\mathrm{Cl}^{-}\left(\mathrm{P}_{\mathrm{K}+/ \mathrm{Cl}-}\right)$ ranging between $\sim 2.5$ to $\sim 10^{28,31,39-41}$. Hydrated $\mathrm{K}^{+}$and $\mathrm{Cl}^{-}$ions ( $\sim 7$ diameter) would pass unobstructed through a $10-12 \AA$ steric constriction site; yet, for all three isoforms the peak energy barriers to $\mathrm{Cl}^{-}$are considerably larger than for $\mathrm{K}^{+}$(Fig. 3d, Extended Data Fig. 8). Peak $\mathrm{Cl}^{-}$barriers localize within the CS region of $\mathrm{Cx} 46_{(\text {Ace) }}$ and $\mathrm{Cx} 50_{\text {(Ace) }}\left(4.8 \mathrm{kcal} \mathrm{mol}^{-1}, \sim 40 \AA\right.$ z-axis; and $4.6 \mathrm{kcal} \mathrm{mol}^{-1}, \sim 38 \AA \mathrm{z}$ axis, respectively), and slightly deeper into the channel pore for $\mathrm{Cx} 26_{(\mathrm{Ace})}$, near the CS-ECV border (4.1 kcal mol ${ }^{-1}, \sim 20 \AA$ z-axis). As a proxy for degree of $\mathrm{P}_{\mathrm{K}+/ \mathrm{Cl}}$ selectivity, we assessed the difference in peak $\mathrm{K}^{+}$and $\mathrm{Cl}^{-}$PMF barriers (DDG = 
$3.2 \mathrm{kcal} \mathrm{mol}^{-1}$ for $\mathrm{Cx} 50_{(\mathrm{Ace})} ; 2.7 \mathrm{kcal} \mathrm{mol}^{-1}$ for $\mathrm{Cx} 46_{(\mathrm{Ace})}$; and $1.6 \mathrm{kcal} \mathrm{mol}^{-1}$ for Cx26(Ace)). These relatively small differences in free energy are in accordance

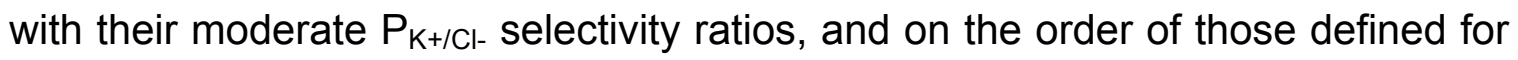
bacterial sodium channels $\left(\sim 3.0-3.5 \mathrm{kcal} \mathrm{mol}^{-1}\right)^{26,27}$, which also display modest ion selectivity $\left(\mathrm{P}_{\mathrm{Na}+/ \mathrm{K}^{+}} \sim 10-30\right)$. In contrast, voltage-gated K-channels display almost ideal selectivity for $\mathrm{K}^{+}$over $\mathrm{Na}^{+}\left(\mathrm{P}_{\mathrm{K}+\mathrm{Na} \mathrm{N}^{+}} \sim 1000\right)$, with energetic barrier differences to these ions reported to be $\sim 6.6 \mathrm{kcal} \mathrm{mol}^{-1}$ for $\mathrm{KcsA}^{25}$.

Diffusion of $\mathrm{Cl}^{-}$ions across the CS energy barriers was relatively rare on the timescale of our equilibrium MD simulations, which necessitated enhanced sampling methods to construct robust $\mathrm{Cl}^{-} \mathrm{PMF}$ calculations (see Methods and Extended Data Fig. 8). Nevertheless, a few $\mathrm{Cl}^{-}$entry events were observed in our simulation data for $\mathrm{Cx} 50$ and $\mathrm{Cx} 46$, and in these cases, $\mathrm{Cl}^{-}$ions appear to comigrate across the high energy barrier of the CS alongside a $\mathrm{K}^{+}$counter ion. It is possible that similar mechanisms involving ionic charge-neutralization enable cation preferring gap junction channels to permit passage of negatively charged signaling molecules (e.g., cAMP and IP3). However, general insights into the mechanism of perm-selectivity are difficult, as ion conductance properties do not always correlate well with the conductance properties of larger molecules ${ }^{41,42}$.

The above analysis supports previous models proposing gap junction substrate selectivity and conductance properties are established by complex mechanisms involving both steric aperture and the unique pattern of electrostatic features 
contributed by isoform-specific amino acid composition ${ }^{42,43}$. In this way, Cx46/50 heteromeric/heterotypic channels confer unique conductance properties of potential functional significance. For example, rectification observed in Cx46/50 heterotypic channels can be explained by the resulting asymmetric free-energy landscape (Extended Data Fig. 8), induced by the uneven distribution of fixed charges ${ }^{30,40}$. Cx46/50 heteromeric assemblies also produced unique $\mathrm{K}^{+} / \mathrm{Cl}^{-} \mathrm{PMF}$ profiles, with peak barriers that were intermediate to their homomeric counterparts (Extended Data Fig. 8), supporting observations made by singlechannel measurement ${ }^{29,44}$.

Additional fine-tuning of gap junction permeation properties may be achieved through co- and/or post-translational modification of pore-lining residues ${ }^{22}$. In our studies, n-terminal acetylation was found to enhance the cation-to-anion specificity of Cx50, Cx46 and Cx26 intercellular channels (Extended Data Fig. 8). Although n-terminal acetylation is irreversible, the effect of this cotranslational modification illustrates how other dynamic and reversible chargemodifying post-translational modifications may serve to spatially and temporally modulate the behavior of inter-cellular communication.

\section{Open-state stabilization of NTH domain}

Despite general similarities, significant differences between the Cx46/50 CryoEM structures and the Cx26 crystal structure are localized to the NTH domain (Fig. 4, Extended Data Fig. 7). The connexin NTH domain contributes to ion 

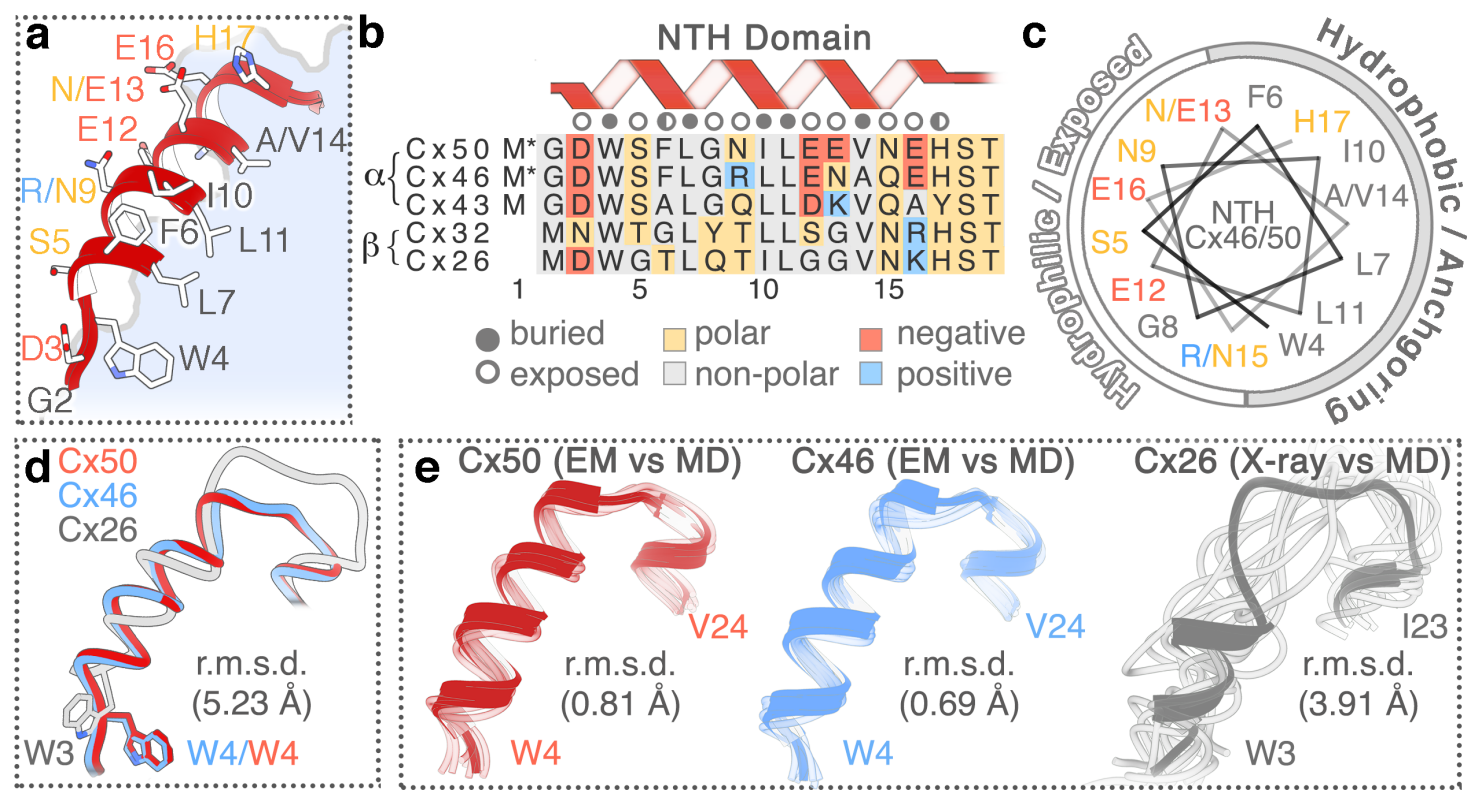

Figure 4. Open-state stabilization of the connexin-46/50 NTH domain. (a) Zoom-view of the Cx46/50 NTH domain. (b) Sequence alignment of the NTH domain from representative a- and b-family connexins. Residues are annotated as buried, exposed, and partially exposed. Asterisk ( $\left.{ }^{*}\right)$ next to M1 indicates co-translational removal confirmed by MS/MS. (c) Helical wheel representation of the $\mathrm{Cx} 46 / 50 \mathrm{NTH}$ domain. In panels a-c, amino acids are labeled for Cx46/50 respectively, and colored by chemical properties (grey hydrophobic, blue - positively charged, red - negatively charged and yellow - hydrophilic). (d) Overlay of NTH domains from experimental structures of Cx50 (red), Cx46 (blue) and Cx26 (grey; PDB 2ZW $3^{10}$ ), after superpositioning of TM1-4 and EC1-2 domains. (e) Superposition of NTH domains of each monomer captured from the MD production (faded tube), and aligned against the initial starting structures, $C \times 50_{(A c e)}$ (left), Cx46 (Ace) $($ center) and Cx26(Ace) (right) displayed as ribbons. $C \alpha$ root mean squared deviation (r.m.s.d.) of the NTH domains are provided in panels $d$ and e.

selectivity and "fast" trans-junctional voltage gating that is common to all connexin isoforms ${ }^{45}$. The NTH folds into the cytoplasmic vestibule where it forms the CS, and is well positioned to function as a selectivity filter/gating domain (Fig. 1d and Fig. 4a). 
In the proposed open-state conformation of $\mathrm{Cx} 46 / 50$ described here, the NTH adopts a regular amphipathic a-helix and ordered loop connecting to TM1 (Fig. 4a-c). The hydrophobic face is established by a set of aromatic and hydrophobic residues that are conserved across various connexin isoforms (W4, L7, I10, L11, and $\mathrm{V} 14$ - in Cx46 and Cx50) (Fig. 4b). These anchoring sites pack against the pore lining helices (TM1/2), and along the interface of neighboring subunits. Despite sequence conservation at these sites, the NTH domain modeled in the crystal structure of Cx26 is in a distinctively different conformation and overall arrangement with respect to the TM domains compared to Cx46/50 (C $\alpha$ r.m.s.d. $=5.2 \AA$, after alignment of the TM and EC domains) (Fig. 4d, Extended Data Fig. 7e). In Cx26, the NTH domain and loop connecting TM1 is less regular, and with the exception of W3 (Cx26 numbering) the conserved hydrophobic residues are modeled toward the solvent ${ }^{10}$.

We propose that the network of hydrophobic anchoring observed in the CryoEM structure of $\mathrm{Cx} 46 / 50$ supports a stabilized open-state conformation. Accordingly, analyses obtained from our MD simulations show the NTH domain for Cx50 and Cx46 is conformationally stable, in both acetylated and non-acetylated states, with only small amplitude backbone fluctuations (r.m.s.f. $\sim 1.0-1.2 \AA$ ) (Fig. 4e, Extended Data Fig. 7). In contrast, the NTH of Cx26 (and Cx26(Ace) $)$ becomes rapidly disordered (i.e., unfolded), and remains conformationally dynamic throughout the production phase of our MD simulations (PDB $2 Z W 3^{10}$ as the starting structure; Fig. 4e, Extended Data Fig. 7). The dynamical behavior of the 
Cx26 NTH domain appears to be consistent with previous MD studies ${ }^{35,46,47}$. The functional significance of these differences in NTH domain structure and dynamic stability is currently unclear. Indeed, instability of the Cx26 NTH domain may be an intrinsic feature. In a more recent X-ray crystallographic study of Cx26 the NTH domain was completely unresolved, presumably due to local disorder ${ }^{11}$. However, potential effects of the conditions required for crystallization may not be ruled out.

The amphipathic nature of the $\mathrm{Cx} 46 / 50$ NTH positions hydrophilic residues implicated in voltage sensing and ion selectivity at the solvent exposed face, forming the cytoplasmic vestibule ${ }^{45}$ (Fig. 4a-c). A network of h-bond interactions appears to contribute to the precise localization of some of these key residues, including the site of $n$-terminal acetylation. The carbonyl group at the acetylatedG2 site appears oriented, at least transiently, through h-bonding to the indole ring of W4 in the same subunit, while in the non-acetylated state $\mathrm{G} 2$ forms a transient inter-molecular ion-pair with D3 of a neighboring subunit (Extended Data Fig. 10). At the same time, the sidechain of D3 is oriented by a relatively stable intramolecular h-bond with the hydroxyl of S5, in both acetylated and non-acetylated forms of both channels (Extended Data Fig. 10). D3 (in Cx46/50; D2 in Cx26) has been identified as a critical site for establishing polarity and/or sensitivity to trans-junctional voltage ${ }^{48-50}$. The precise spatial orientation imposed by these interactions may contribute to the strict conservation at this site, as replacement of D3 to a similarly negative charged residue, glutamate, results in significant 
perturbation to gating properties, conductance and free energy of the openclosed state in Cx50 gap junctions ${ }^{51}$.

\section{Mutational Hot Spots Linked to Hereditary Cataracts}

Cx46/50 gap junctions serve a critical role in maintaining the transparency of the eye lens by establishing a pathway for water, ion and nutrient circulation and removal of metabolic waste in this avascular organ ${ }^{12}$. Consequently, a variety of human genetic variations in $\mathrm{Cx} 50$ and Cx46 have been linked to hereditary cataract formation ${ }^{52}$. Age-related cataracts are currently incurable (except by surgery) and remain the leading cause of blindness in the world ${ }^{53}$. The more rare congenital forms of this disease have been linked to genetic mutation of various lens proteins, including Cx46/50 - offering critical insight into the mechanisms of maintaining lens transparency throughout life ${ }^{54}$. We mapped 46 mutation sites in Cx46/50 linked to congenital cataracts currently reported on the Cat-Map database $^{54}$ (Fig. 5a). This analysis rationalizes many of the disease-causing effects induced by these polymorphisms, as identified mutational hot-spots localize to functionally important regions of the $\mathrm{Cx} 46 / 50$ gap junction structure. These include a cluster of residues localized within the EC2 docking site (e.g., Cx46 N188T/l and Cx50 R189Q/W), and at regions that deviate significantly from the Cx26 structure, such as the NTH gating/selectivity domain (e.g., Cx46 G2D and $\mathrm{D} 3 \mathrm{Y} / \mathrm{H}$ and $\mathrm{Cx} 50 \mathrm{L7P}$ ) and several sites localized to the TM1/2 pore lining helix that form an interaction-network with the NTH domain, where mutation is expected to affect the permeation pathway or impact folding and stability within 

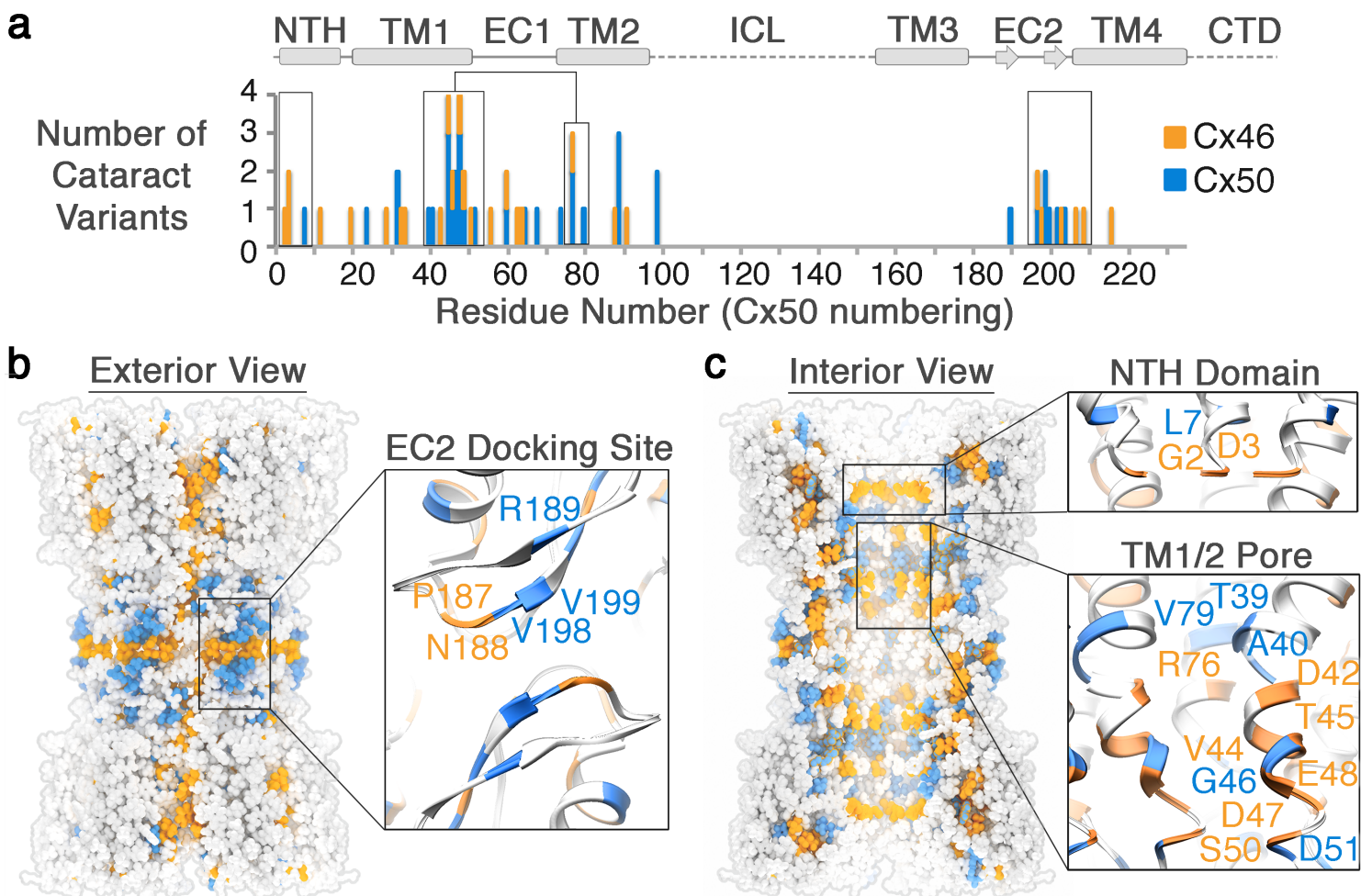

Figure 5. Mutational hot-spots in connexin-46/50 linked to congenital cataracts. (a) Histogram of connexin-46/50 genetic variants linked to hereditary (congenital) cataracts. Sites of Cx46 (orange) and Cx50 (blue) mutation are overlaid using Cx50 amino acid numbering, with secondary structure and domain elements indicated. Regions of high genetic variation "hot-spots" within the NTH, TM1/2 and EC2 domains are boxed. (b) Exterior view and (c) interior view of the $C \times 46 / 50$ gap junction channel with cataract mutation sites mapped for Cx46 (orange) and Cx50 (blue), with zoom-views of the EC2 domain (panel $b$, inset), the NTH domain (panel $c$, top inset) and TM1/2 pore lining helices (panel $c$, bottom inset), with representative mutation sites labeled.

these regions of the protein (Fig. $\mathbf{5 b}, \mathbf{c}$ ). The localization of disease-causing mutations underscores the functional significance of these core structural/functional elements, and the importance of proper cell-to-cell communication through $\mathrm{C} \times 46 / 50$ gap junctions for the maintenance of lens transparency. The ability of CryoEM to provide high-resolution structural information on gap junctions may finally open the door to detailed mechanistic 
investigation of these disease-causing mutations in $C \times 46 / 50$ and in other isoforms responsible for a diverse range of connexin-opathies.

\section{Acknowledgements}

We thank Tamir Gonen for early support of this work, and Tom White, Larry David, Upendra Adhikari and Barmak Mostofian for helpful discussions. We are grateful to the staff at the OHSU Multiscale Microscopy Core and Advanced Computing Center, and to William Garrick (PSU) for their assistance and training. C.V.R. and D.S.C. are supported by funding from the European Research Council (No. 695511-ENABLE). D.M.Z. and C.C.Y. are supported by the Center for Spatial Systems Biomedicine at OHSU. S.L.R. is supported by the Medical Research Foundation of Oregon and the National Institutes of Health (R35GM124779).

\section{Author Contributions}

J.B.M. and B.G.H. contributed equally. J.B.M. and C.C.Y. collected the CryoEM datasets. J.B.M. performed image processing and atomic modeling of the Cx46/50. S.E.O. conducted protein purification and negative stain EM studies. B.G.H. conducted and analyzed the MD simulations. D.S.C conducted the crosslinking studies and MS/MS analysis. D.M.Z. contributed to the experimental design and statistical analysis of the MD simulations. All authors contributed to manuscript preparation. S.L.R. provided overall guidance to the design and execution of this work. 


\section{Author Information}

These authors contributed equally: Janette B. Myers and Bassam G. Haddad.

\section{Affiliations}

Department of Chemistry, Portland State University, Portland OR 97201, U.S.A. Janette B. Myers, Bassam G. Haddad, Susan E. O’Neill and Steve L. Reichow

Department of Biomedical Engineering, Oregon Health and Sciences University, Portland OR 97239, U.S.A.

Craig C. Yoshioka and Daniel M. Zuckerman

Physical and Theoretical Chemistry Laboratory, University of Oxford, Oxford OX1 3QZ, U.K.

Dror S. Chorev and Carol V. Robinson

\section{Competing Interest}

The authors declare no competing interests.

\section{Corresponding Author}

Correspondence and requests for materials should be addressed to reichow@pdx.edu

Reprints and permissions information is available at www.nature.com/reprints 
Methods

Cx46/50 purification and amphipol reconstitution. Lamb eyes were obtained from the Wolverine Packers slaughterhouse (Detroit, MI), and the lenses were removed using a surgical blade and stored at $-86^{\circ} \mathrm{C}$. Gap junction intercellular channels were isolated from the core lens fiber tissue, containing c-terminal truncation variants of $\mathrm{C} \times 46$ and $\mathrm{Cx} 50$ (a.k.a. MP38) ${ }^{16,55-57}$ (Extended Data Fig. 1). Details of the purification procedure are provided below.

Core lens fiber cell tissue was dissected from cortical tissue using a surgical blade, and stripped membranes were prepared as described ${ }^{58-60}$. Total protein concentration was determined by BCA (Pierce) and membranes were stored at $86^{\circ} \mathrm{C}$ suspended in storage buffer containing $10 \mathrm{mM}$ Tris-8.0, $2 \mathrm{mM}$ EDTA, 2 mM EGTA, at a total protein concentration of $\sim 2 \mathrm{mg} \mathrm{mL}^{-1}$. Stripped membranes were thawed from $-86^{\circ} \mathrm{C}$ and solubilized in $10 \mathrm{mM}$ Tris-8.0, $2 \mathrm{mM}$ EDTA, $2 \mathrm{mM}$ EGTA, $1 \%\left(w\right.$ vol $\left.^{-1}\right)$ n-Decyl- $\beta$-D-Maltoside $(\mathrm{DM})$ for 30 minutes at $37^{\circ} \mathrm{C}$. Unsolubilzed debris was cleared by ultra-centrifugation at $150,000 \times \mathrm{g}$ for 30 minutes at $4^{\circ} \mathrm{C}$. The solubilized fraction was applied to an anion-exchange chromatography column (UnoQ, BioRad) equilibrated with buffer A, containing 10 $\mathrm{mM}$ Tris $\mathrm{pH}$ 8.0, $2 \mathrm{mM}$ EDTA, $2 \mathrm{mM}$ EGTA, 0.3\% DM (wt vol ${ }^{-1}$ ). Protein was eluted with buffer B that additionally contained $500 \mathrm{mM} \mathrm{NaCl}$. Elution peaks containing $\mathrm{C} \times 46 / 50$, as determined by SDS-PAGE, were pooled and applied to a size exclusion chromatography (SEC) column (ENC650, BioRad) equilibrated with SEC buffer, containing $20 \mathrm{mM}$ HEPES, $150 \mathrm{mM} \mathrm{NaCl}$, 2mM EDTA, 2mM 
EGTA and $0.3 \%$ DM $\left(w\right.$ vol $\left.^{-1}\right)$. Peak fractions containing purified $\mathrm{C} \times 46 / 50$ were pooled and protein concentration was determined by UV absorbance. All chromatography steps were performed at $4^{\circ} \mathrm{C}$. The presence of both $\mathrm{C} \times 46$ and Cx50 was confirmed by western blot analysis using polyclonal antibodies directed against the n-terminal domain of Cx46 (AP11570PU-N, Acris) and cterminal domain of Cx50 (sc-50432, Santa Cruz) (Extended Data Fig. 1a) and by mass-spectrometry analysis, described below.

Purified Cx46/50 was exchanged from DM to Amphipol A8-35 (Anatrace), as follows. Amphipol was added to freshly purified protein in an 5:1 amphipol:protein $\left(\mathrm{wt} \mathrm{vol}^{-1}\right)$ ratio using a stock solution prepared at $5 \%\left(\mathrm{wt} \mathrm{vol}^{-1}\right)$ in water. This mixture was incubated for 2.5 hours at $4^{\circ} \mathrm{C}$, while rotating. Detergent was then removed by application of SM-2 Biobeads (BioRad) at a ratio of 30:1 (wt wt ${ }^{-1}$ ) beads:detergent. Biobeads were incubated overnight at $4^{\circ} \mathrm{C}$, while rotating. Biobeads were then removed by running samples over a polyprep column (BioRad) that had been washed with detergent-free SEC buffer (20 mM HEPES $\mathrm{pH}$ 7.4, $150 \mathrm{mM} \mathrm{NaCl}, 2 \mathrm{mM}$ EDTA, 2 mM EGTA). Samples were further cleared by ultra-centrifugation at $150,000 \times \mathrm{g}$ for $20 \mathrm{~min}$ at $4^{\circ} \mathrm{C}$. The clarified sample was then applied to an SEC column (ENC650, BioRad) equilibrated with detergentfree SEC buffer to remove excess amphipol. Peak fractions corresponding to amphipol stabilized Cx46/50 were pooled and concentrated for single particle EM studies (Extended Data Fig. 1b). Final protein concentration was determined by UV absorbance at $280 \mathrm{~nm}$. 
Chemical cross-linking and mass-spectrometry. Cx46/50 was prepared for cross-linking and mass-spectrometry analysis in order to confirm heteromeric assembly of the two lens isoforms (Extended Data Fig. 1d-f). Crosslinking was carried out using the amine-to-amine cross-linking reagents bis(sulfosuccinimidyl)suberate (BS3; Thermo Fischer Scientific) and disuccinimidyl suberate (DSS H12:D12; Creative Molecules). For BS3 crosslinking, $0.5 \mu \mathrm{L}$ of $12.5 \mathrm{mM}$ BS3 dissolved in deuterium-depleted water (DDW) were added to $10 \mu \mathrm{L}$ of purified $20 \mu \mathrm{M} \mathrm{Cx} 46 / 50$ complexes and incubated 2 hours on ice. For DSS cross-linking, $0.5 \mu \mathrm{L}$ of a $1: 1$ mixture of $25 \mathrm{mM}$ nondeuterated $(\mathrm{d} 0)$ and deuterated (d12) DSS cross-linker dissolved in DMSO was added to $10 \mu \mathrm{L}$ purified $20 \mu \mathrm{M} \mathrm{Cx} 46 / 50$ complexes. A control sample, in which only DMSO or DDW were added to the proteins was also done. DSS cross-linked samples were incubated at room temperature for 2 hours in a thermomixer at 300 rpm, while BS3 cross-linked samples were incubated for 2 hours on ice. The cross-linking reaction was quenched by adding Tris $\mathrm{pH}=7.4$ at a final concentration of $100 \mathrm{mM}$ for 15 minutes at room temperature in a thermomixer at 300 rpm.

The quenched reaction mixtures were separated on a NuPAGE gel (Thermo Fischer Scientific) and protein bands stained with InstantBlue (Expedeon). Crosslinked protein bands were excised and digested with trypsin (Promega) as described ${ }^{61}$. Peptides were re-suspended in $0.1 \%$ Formic Acid and separated on 
an Ultimate 3000 UHPLC system (Thermo Fischer Scientific) and electrosprayed directly into a QExactive mass-spectrometer (Thermo Fischer Scientific) through an EASY-Spray nano-electrospray ion source (Thermo Fischer Scientific). The peptides were trapped on a C18 PepMap100 pre-column $(300 \mu \mathrm{m}$ i.d. x $5 \mathrm{~mm}$, $100 \AA$, Thermo Fisher Scientific) using solvent A ( $0.1 \%$ Formic Acid in water) at a pressure of 500 bar. The peptides were separated on an in-house packed analytical column (75 $\mu \mathrm{m}$ i.d. packed with ReproSil-Pur 120 C18-AQ, $1.9 \mu \mathrm{m}, 120$ $\AA$, Dr.Maisch $\mathrm{GmbH}$ ) using a gradient (length:50 minutes, $15 \%$ to $38 \%$ for $30 \mathrm{~min}$ followed by $38 \%$ to $58 \%$ solvent B ( $0.1 \%$ formic acid in acetonitrile, flow rate: 200 $\mathrm{nL} / \mathrm{min}$ ) for $15 \mathrm{~min}$. The raw data was acquired on the mass-spectrometer in a data-dependent mode (DDA). Full scan MS spectra were acquired in the Orbitrap (scan range $350-2000 \mathrm{~m} / \mathrm{z}$, resolution 70000 , AGC target $3 \mathrm{e} 6$, maximum injection time $50 \mathrm{~ms}$ ). After the MS scans, the 10 most intense peaks were selected for HCD fragmentation at $30 \%$ of normalized collision energy. HCD spectra were also acquired in the Orbitrap (resolution 17500, AGC target 5e4, maximum injection time $120 \mathrm{~ms}$ ) with first fixed mass at $180 \mathrm{~m} / \mathrm{z}$. Charge exclusion was selected for $1+$ and $2+$ ions. The dynamic exclusion set to 5 seconds. Crosslinking identification and analysis was done using $\mathrm{pLink}^{62}$ and Xcalibur 2.2 (Thermo Scientific). All peptides were manually validated.

For identification of proteins and post-translational modifications, protein bands were excised from the gel and processed as described above. Massspectrometry analysis was carried out similarly with a gradient of $15-38 \%$ for 30 
min and the Orbitrap set to $350-1500 \mathrm{~m} / \mathrm{Z}$. Charge exclusion was selected for $1+$ and unassigned ions, dynamic exclusion was set to 5s. PTM identification was done using the MASCOT Daemon client program.

Negative stain electron microscopy. Amphipol stabilized Cx46/50 was prepared for negative stain EM as described ${ }^{58,63}$. Briefly, a $3 \mu \mathrm{L}$ drop of sample $\left(\sim 0.02 \mathrm{mg} \mathrm{mL}^{-1}\right)$ was applied to a glow-discharged continuous carbon coated EM specimen grid (Ted Pella), blotted with filter paper and washed two times with detergent-free SEC buffer. The specimen was then stained with freshly prepared $0.75 \%\left(\right.$ wt vol $\left.^{-1}\right)$ uranyl formate (SPI-Chem).

Negatively stained specimens were visualized on a $120 \mathrm{kV}$ TEM (iCorr, FEI) at a nominal magnification of 49,000x at the specimen level (Extended Data Fig. 1c). Digital micrographs were recorded on a $2 \mathrm{~K} \times 2 \mathrm{~K}$ CCD camera (FEI Eagle) with a calibrated pixel size of $4.37 \AA$. A total of 75 micrographs were collected. CTF parameters were determined in EMAN2 ${ }^{64}$ and micrographs free of significant astigmatism and drift were selected based on Thon rings in the power spectra. A total of 5,330 particles were hand selected in EMAN2 and extracted with a box size of $84 \times 84$ pixels. Reference-free 2D class averages were generated using CTF-corrected (phase-flipped) images without applied symmetry (Extended Data Fig. 1c). A subset of 3,952 'good' particles was selected following multiple rounds of 2D classification, and an initial model was generated de novo in EMAN2 using a subset of 12 class averages as input. This model was refined 
against the 'good' particle image dataset in EMAN2 with applied D6 symmetry to a final resolution of $\sim 20 \AA$ (Extended Data Fig. 2).

CryoEM data collection, image processing and 3D reconstruction. Samples were prepared for CryoEM by applying $5 \mu \mathrm{L}$ of amphipol-stabilized Cx46/50 (2.35 $\mathrm{mg} \mathrm{mL}^{-1}$ ) to a glow-discharged holey carbon grid (Quantifoil $\mathrm{R}$ 1.2/1.3) for 10 seconds. The grid was blotted for 4.0 seconds and plunge frozen in liquid ethane using a Vitrobot $(\mathrm{FEI})$ at $100 \%$ humidity and stored under liquid nitrogen.

CryoEM specimen grids were imaged on a Titan Krios (FEI) operated at $300 \mathrm{kV}$. Image stacks were recorded using a K2 summit direct electron detector (Gatan) in counting mode with a super-resolution pixel size of $0.665 \AA / p i x$. The dose rate was 3.2 electrons pixel ${ }^{-1} \mathrm{sec}^{-1}$, with 4 frames per second collected for a total exposure time of 10 seconds. A Gatan energy filter with a slit width of $30 \mathrm{eV}$ was used during data collection. An initial dataset of 1104 micrographs (Dataset 1) was obtained by automated data collected using SerialEM ${ }^{65}$, with nominal defocus values from $1.25-2.5 \mu \mathrm{m}$.

Drift correction and dose weighting was performed using MotionCor ${ }^{66}$ and CTF correction was performed using GCTF $^{67}$. 261,206 particles were picked from Dataset 1 using DoGPicker ${ }^{68}$. Particles were extracted with $2 x$ binning (resulting in a pixel size of $1.3 \AA /$ pix). Five rounds of $2 \mathrm{D}$ classification in Relion $2.0^{69}$ left 53,791 "good" particles. These particles were then subjected to 3D classification 
in Relion with four classes and no imposed symmetry. The most populated class contained 33,967 particles. These particles were unbinned and another round of 3D classification was performed, reducing the population of particles to 30,128 . 3D auto-refinement was then performed on this set of particles with D6 symmetry imposed. After masking and post processing in Relion, the final map had a resolution of $3.4 \AA$ by gold standard FSC (Extended Data Fig. 2 and Extended Data Fig. 3a).

An additional 1093 micrograph data set was collected and processed as above. Particles were picked from this set with DoGPicker, $2 x$ binned and pooled with the original set of 261,206 particles for a total of 398,066 particles (Dataset 2). A set of 66,480 "good" particles was obtained after five rounds of 2D classification. These particles were subjected to $3 \mathrm{D}$ classification with four classes and no imposed symmetry. The most populated class contained 55,475 particles. This dataset was further culled by removing particles extracted from micrographs with Thon rings that did not extend beyond $3.5 \AA$, resulting in a final dataset of 44,547 particles. These particle images were subjected to 3D auto-refinement in Relion with D6 symmetry, resulting in an overall 3.5 A resolution 3D reconstruction after post-processing as judged by gold-standard FSC (Extended Data Fig. 3b). Local resolution analysis using BlocRes ${ }^{70}$ showed the $3.4 \AA$ map possessed exceptionally high resolution features within the central regions of the structure, while the $3.5 \AA$ reconstruction contained more uniformly defined features throughout the density map, consistent with visual inspection (Extended Data 
Fig. 3c-d). An overview of CryoEM data collection and 3D refinement statistics is provided in Extended Data Fig. 2.

Cx46/50 symmetry analysis. In an attempt to uncover a specific pattern(s) of Cx46/50 heteromeric/heterotypic co-assembly, 3D auto-refinement was also pursued in Relion using C1, C3, C6, and D3 symmetries, using the final 30,128particle (Dataset 1) and $3.4 \AA$ map (filtered to $15 \AA$ ) as input. These refinements converged to $4.1 \AA$ (C1), $3.9 \AA(C 3)$, and $3.7 \AA$ (D3 and C6). Examination of the resulting maps provided no indication that the $\mathrm{Cx} 46 / \mathrm{C} \times 50$ subunits were being separately resolved (Extended Data Fig. 4,5). Further attempts were performed using 3D classification in Relion with C3, C6, and D3 symmetry, using the larger 55,475 particle set (which had already been subjected to one round of 3D classification with no imposed symmetry). The initial model was the $3.4 \AA$ map filtered to $25 \AA$. No resolution limit was enforced, and classification was attempted with and without image alignment. Some classifications converged to a single class, while some maintained a more even distribution of particles throughout 3D classification. 3D auto-refine was attempted with the most populated class from each attempted symmetry group. C3 symmetry refined to $3.9 \AA$ from a set of 47,074 particles; C6 symmetry refined to $3.8 \AA$ from a set of 38,404 particles; D3 symmetry refined to $4.2 \AA$ from a set of 16,520 particles. Inspection of the resulting maps provided no indication that isoform-specific features were being separately resolved into any specific symmetric arrangements (not shown). Finally, focused refinement strategies with signal 
subtraction were also explored using Relion, by masking a single hemi-channel or just a single subunit. However, these procedures did not produce isoformspecific features, or improved results compared to the D6 symmetrized maps.

As we were unable to identify a specific pattern of co-assembly for the Cx46/50 dodecameric channel, all further analysis and model building was performed using the 3D maps generated with imposed D6 symmetry. Both pre-processed and post-processed maps and associated masks generated from Datasets 1 and 2 have been deposited to the electron microscopy data bank (EMD-9116).

Atomic modeling, refinement and validation. The post-processed maps obtained with D6 symmetry were used to build and stereochemically refine atomic models for both $\mathrm{C} \times 46$ and $\mathrm{C} \times 50$, following similar procedures. An initial $\mathrm{C} \alpha$ model was generated using the available crystal structure of connexin-26 $\left(\mathrm{PDB} 2 \mathrm{ZW} 3^{10}\right.$ ) and placed into the post-processed $3.4 \AA$ density map using rigidbody fitting. Starting from this template, all atom models of Cx46 and Cx50 were built separately into the CryoEM density using $\mathrm{COOT}^{71}$. Disulfide bonds were modeled for Cx 50 (C54-C201, C61-C195 and C65-C190) and Cx46 (C54C189, C61-C183 and C65-C178). Models were subjected to real space refinement in Phenix ${ }^{72}$ with non-crystallographic symmetry (D6-symmetry) and secondary structure restraints imposed. Successive rounds of modeling and refinement were conducted until refinement statistics converged, as judged by Molprobity ${ }^{73}$ (Extended Data Fig. 2). The FSC of the model versus map dropped 
below 0.5 at $3.4 \AA$ (Dataset 1) and $3.5 \AA$ (Dataset 2) for both $C x 46$ and $C x 50$, judged by the output of Phenix real space refine (Extended Data Fig. 3a-b). The NTH domain of Cx46 and Cx50 (residues $2-20$ ) were further refined using the post-processed $3.5 \AA$ density map (Dataset 2 ), as this region of the map was more well defined compared to the original $3.4 \AA$ map. Over areas of the density maps where the sequence of $\mathrm{Cx} 46$ and $\mathrm{Cx} 50$ are identical or similar (80\% identical and $8 \%$ similar) both models fit well into the D6 symmetrized map, and these regions tend to display well-resolved sidechain density. Over regions where the sequence of $\mathrm{Cx} 46$ and Cx50 differ, sidechain density is sometimes weaker. This observation is possibly due to the imposed D6 symmetry averaging the density of two different sidechains in these areas, or relative flexibility as many of these residues contain solvent exposed sidechains. In these areas of difference, where EM density is observed, both Cx46 and Cx50 can be fit into the density equally well (Extended Data Fig. 4,5). Fit of the models to the CryoEM density map were assessed quantitatively by local resolution analysis using BlocRes $^{70}$, comparing the calculate maps of $\mathrm{Cx} 50$ and $\mathrm{C} \times 46$ atomic models to the 3.4 $\AA$ experimental CryoEM map (Extended Data Fig. 3). This analysis was tabulated by assigning each residue a range of resolution values corresponding to the output of this analysis, including the alpha carbon and extending to the end of the sidechain (Supplementary Table 2,3).

Completed models of the dodecameric Cx46 and Cx50 structures, corresponding to residues $2-97 ; 142-222$ and $2-97 ; 154-234$, respectively, have been 
deposited to the protein data bank (PDB ID's 6MHQ and 6MHY, respectively). Additional density is observed for the region of TM2 that extends toward the cytoplasm; however, we did not model this region (corresponding to $\sim 1-2$ turns of an $\alpha$-helix) due to the lack of identifiable sidechain density. Various heterotypic/heteromeric models of $\mathrm{Cx} 46 / 50$ were generated for analysis by applying appropriate symmetry operations to the monomeric subunits and combined to form a complete gap junction structure. Coulombic surface potentials were calculated and displayed using Chimera ${ }^{74}$.

Molecular dynamics simulations. Visual Molecular Dynamics (VMD) v1.9.3 was used to build systems for Cx50, Cx46, Cx46/50 heteromeric and heterotypic models, and Cx26 (PDB 2ZW3) ${ }^{10}$. Representative Cx46/50 heteromeric models (heteromeric models I and II, with C3 or D3 point group symmetry, respectively) were constructed by applying the appropriate symmetry operations to the coordinates of the individual subunits. The Cx46/50 heteromeric and heterotypic channels were run through a steepest decent minimization routine using Phenix ${ }^{72}$ to ensure no clashes were introduced in the preparation of these models. Each system comprised the full dodecameric gap junction, and was prepared in explicit solvent and embedded in two lipid bilayers composed of 1-palmitoyl-2-oleoyl-snglycero-3-phosphocholine (POPC), mimicking a cell-cell junction. The Cx26 crystal structure was prepared for MD by completing the sidechains at residues Lys15, Ser17 and Ser19 and missing protons were added to all amino acids at standard positions. Sidechains were protonated according to neutral conditions, 
and the HSD model was used for all histidine residues. To facilitate comparison to the $\mathrm{Cx} 46 / 50$ models, the $\mathrm{C} \times 26$ model was constructed with a Met1 residue added, which was missing in the published crystal structure, but expected to be present in the protein based on proteomic analysis ${ }^{76}$, as previously described ${ }^{47}$. Disulfide bonds identified in the experimental structures were enforced for Cx50 and Cx46 (as described above), and for $\mathrm{C}^{2} 26^{10}$ (C53-C180, C60-C174 and C64-C169). Amino acids corresponding to the intracellular loop (ICL) connecting TM2/TM3, and c-terminal domain (CTD) of Cx50, Cx46 and Cx26 and were not included for MD simulation, as experimental data describing the structure of these large domains ( $\sim 50$ residue ICL and $\sim 200$ residue CTD in Cx46/50) are missing. The introduced $\mathrm{n}$ - and c-terminal residues resulting from the missing ICL segment (Cx46 L97 and L142; Cx50 V97 and L154; and Cx26 G109 and K125) were neutralized. N-terminal acetylation sites were introduced in VMD through an all-atom acetylation patch in the automated PSF-Builder. A complete list of modeled residues for each system is provided in Supplementary Tables 1.

The prepared protein structures were submerged in a hydration shell using Solvate $1.0 .1^{77}$. Water was removed from sections of the channel corresponding to transmembrane domains, based on hydrophobic character and localization of amphipol observed in the experimental CryoEM data ( 20-50 $\AA$ from the center of the channel). The VMD membrane-builder plugin was used to add two POPC bilayers, with dimensions of $152 \times 152 \AA$ for $\mathrm{C} \times 46, \mathrm{C} \times 50$ and $\mathrm{C} \times 46 / 50$ models, and $155 \times 155 \AA$ for Cx26 and lipids overlapping with protein were removed. The 
entire system was then placed in a water box with dimensions $150 \times 150 \times 180 \AA$ for Cx46, Cx50 and Cx46/50 models, and $150 \times 150 \times 183 \AA$ for Cx26, using VMD's Solvate plugin. The system was neutralized using the Autoionize plugin, then $150 \mathrm{mM} \mathrm{KCl}$ and $150 \mathrm{mM} \mathrm{NaCl}$ were added to the solvent areas corresponding to intracellular and extracellular regions of the simulation box, respectively (see Fig. 3c). A summary of atoms counts for each system is provided in Supplementary Table 1.

GPU-accelerated nanoscale molecular dynamics (NAMD) $2.12^{78}$ was used for all classical MD simulations, using the CHARMM36 force-field ${ }^{79,80}$ for all atoms and TIP3P explicit model for water. Each system was prepared following the same minimization and equilibration protocol, as follows. An initial minimization of the lipid-tails, with all other atoms fixed, was performed for $1 \mathrm{~ns}$ with a $1 \mathrm{fs}$ time-step, allowing the tails to 'melt'. Next, the system, including lipids, solvent and ions were allowed to minimize around the protein, with the protein harmonically constrained for 1 ns. For the Cx46/50 heteromeric/heterotypic and acetylated models, a second minimization step was applied, where the system was free to minimize with a harmonic constraint on the protein backbone to ensure stable quaternary structure. The entire system was then released from restraints and subjected to all-atom equilibration runs employing Langevin thermostat, with a constant temperature of $310 \mathrm{~K}$ and constant pressure of $1 \mathrm{~atm}$, with 1 or $2 \mathrm{fs}$ time-steps and allowed to proceed for 30 ns (see Supplementary Table 1). Periodic boundary conditions were used to allow for the particle mesh Ewald 
(PME) calculation of electrostatics. Finally, all of the models were continued for a minimum of 50 ns of production. Root mean squared deviations (r.m.s.d.) and root mean squared fluctuations (r.m.s.f.) were calculated using VMD. All three gap junctions approached a steady r.m.s.d. within 20 ns of the equilibration phase (Extended Data Fig. 7a-b). All of these systems maintained an electrochemical seal to extracellular sodium ions $\left(\mathrm{Na}^{+}\right)$during $\mathrm{MD}$ simulation (e.g., Fig. 3c), validating the stability of inter-molecular docking-site interactions and the various heteromeric/heterotypic models generated for analysis.

Calculation of the potential-of-mean-force (PMF) with respect to $\mathrm{K}^{+}$and $\mathrm{Cl}^{-}$was performed using the fundamental principle of detailed balance via a onedimensional Markov State Model (MSM). Configuration space was subdivided based on a natural coordinate, the channel pore (z-axis), and segmented into bins of $4 \AA$ in length. Using a lag-time of 2 ps, a transition matrix was calculated from the trajectories of individual ions within the simulation. The $i \rightarrow j$ transition probability $k_{i, j}$ is computed via Equation 1 :

$$
k_{i, j} \cong \frac{N_{i, j}}{N_{i}}
$$

Where $N_{i, j}$ is the count of transitions during the lag interval and $N_{i}$ is the count of ions in bin $i$ at the beginning of each lag interval. PMFs were constructed using the principal of detailed balance:

$$
P_{i}^{\mathrm{eq}} k_{i, i+1}=P_{i+1}^{\mathrm{eq}} k_{i+1, i} \quad \text { Equation (2) }
$$




$$
\begin{array}{ll}
e^{\frac{-\Delta G_{i, i+1}}{R T}}=\frac{P_{i+1}^{\mathrm{eq}}}{P_{i}^{\mathrm{eq}}}=\frac{k_{i, i+1}}{k_{i+1, i}} & \text { Equation (3) } \\
\mathrm{PMF}(i)=\Delta G(i)=\sum_{n=1}^{i-1}-R T \ln \left(\frac{k_{n, n+1}}{k_{n+1, n}}\right) & \text { Equation (4) }
\end{array}
$$

Here, $P^{e q}$ are the equilibrium probabilities for an ion to occupy each bin respectively (Equation

2), $\Delta G_{i, i+1}$ is the free energy difference from bin $i$ to $i+1, \mathrm{R}$ is the gas constant (1.986 Cal mol$\left.{ }^{-1} \mathrm{~K}^{-1}\right)$, and $\mathrm{T}$ is temperature $(310 \mathrm{~K})$ (Equation 3,4). Final PMF values were adjusted so that the values of the bulk solvent where zero. PMF curves in Fig. 3 and Extended Data Fig. 7 were derived by mapping $z$ values to the corresponding bin index $i$ and subsequently smoothed using MS Excel. The detailed-balance (rates-based) approach is justified by the high mobility of ions within the channel pore, and the time-scales used for analysis were validated by assessing the convergence of the unsymmetrized data to the symmetrized values presented in Fig. 3d and Extended Data Fig. 8. The results were shown to closely match PMFs constructed by taking the population profile, or average counts $\left(<\left(N_{i}\right)>\right.$ ), of the $\mathrm{K}^{+}$ions along the channel pore (z-axis) and solving: $\left.\Delta G=-R T \ln \left(<\left(N_{i}\right)\right\rangle\right)^{11,81,82}$ (Extended Data Fig. 8d).

Because the detailed-balance approach requires only local equilibrium sampling, we were able to apply a distributed seeding protocol to construct PMFs for $\mathrm{Cl}$ ions. Initial analysis of $\mathrm{Cl}^{-}$trajectories revealed this ion to be poorly sampled inside the channel pore of the $\mathrm{C} \times 50, \mathrm{C} \times 46$ and $\mathrm{C} \times 26$ models, presumably due to 
an energetic barrier presented by significant regions of negative coulombic potential for each of these systems (see Fig. 3A). Therefore, a distributed seeding approach was employed where a single $\mathrm{Cl}^{-}$ion was randomly introduced (seeded) by replacing a $\mathrm{K}^{+}$ion within the pore of the equilibrated channel. These coordinates were energy minimized and initial velocities were randomized before allowing the simulation to proceed for $10 \mathrm{~ns}$. This procedure was repeated for each system 3-16 times until sufficient sampling was achieved, as determined by monitoring the resulting PMFs. $\mathrm{Cl}^{-}$PMFs were constructed based on the transition rates (as described above), which is not sensitive to the initial placement of the ion. This seeding approach was validated by showing the resulting PMF recapitulated the features of a $\mathrm{Cl}^{-} \mathrm{PMF}$ obtained for $\mathrm{Cx} 46$, where sufficient sampling had been achieved through random diffusion (Extended Data

Fig. 8e). Trajectories from these distributed seeding simulations were combined with the production phase data and included in the MSM for calculation of final $\mathrm{Cl}^{-} \mathrm{PMFs}$.

Analysis of hydrogen bonding within the NTH domains of Cx46 and Cx50 models was performed by recording the distance vs. time of potential donor-acceptor pairs. The three sets of interactions probed were potential inter-molecular hydrogen bonds between D3 and the neighboring n-terminal $\mathrm{G} 2$ residue, the intra-molecular hydrogen bonding between D3 and S5, and the intra-molecular hydrogen bonds between N-term acetyl and W4 for acetylated models of Cx46 and Cx50. To simplify the analysis of hydrogen bonding interactions involving 
equivalent rotameric donor-acceptor configurations, heavy atoms were selected for D3 $\left(\mathrm{C}_{\mathrm{\gamma}}\right)$ and $\mathrm{G} 2(\mathrm{~N})$ for analysis (Extended Data Fig. 10). For comparison, equivalent analysis for Cx26 was conducted (between D2 and M1 and T5 of the adjacent subunit). For Cx26, D2 appeared to form intra-molecular hydrogen bonding pairing with $\mathrm{T} 5$; however, stable inter-molecular pairing interactions with Met1 or T5 (as indicated in the crystal structure ${ }^{10}$ ) were not identified during the production phase of these simulations (data not shown), as previously reported ${ }^{35}$.

The NTH domain of Cx26 was found to be unstable (i.e., rapid unfolding) during MD simulation in either the acetylated or non-acetylated states (Extended Data Fig. 7 and Fig. 4e). The significance for this dynamical behavior is not clear; however, we attribute this feature to be a potential reason for the variation of our calculated PMFs of Cx26 compared to previous studies ${ }^{47}$. Notably, Kwon et al. reported the Cx26 channel to be anion selective in the absence of $n$-terminal acetylation ${ }^{47}$. These authors conducted an elegant set of experiments employing a GCMC/BD based approach for modeling ion conductance using a model of the Cx26 hemi-channel (by extracting a single hexamer of the Cx26 intercellular channel). However, a limitation of GCMC/BD method is the protein structure is held static, and as such the resulting PMFs obtained by this approach would be significantly influenced by the selected conformational state of the Cx26 NTH domain. These caveats should be considered when comparing results presented in this work. 
Statistical analysis. 95\% confidence intervals for comparison of C $\alpha$ r.m.s.f. values were calculated using a two-tailed student t-test. No statistical methods were used to predetermine sample size for the CryoEM datasets. The experiments were not randomized, and investigators were not blinded to allocation during experiments and outcome assessment.

\section{Data Availability}

CryoEM density maps have been deposited to the Electron Microscopy Data Bank (EMD-9116). Coordinates for Cx46 and Cx50 atomic models have been deposited to the Protein Data Bank (6MHQ and 6MHY). The original multi-frame micrographs have been deposited to EMPIAR (EMPIAR-10212). 


\section{Extended Data Figures}

a

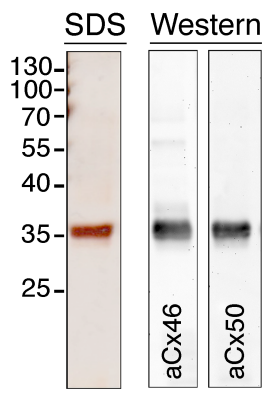

b

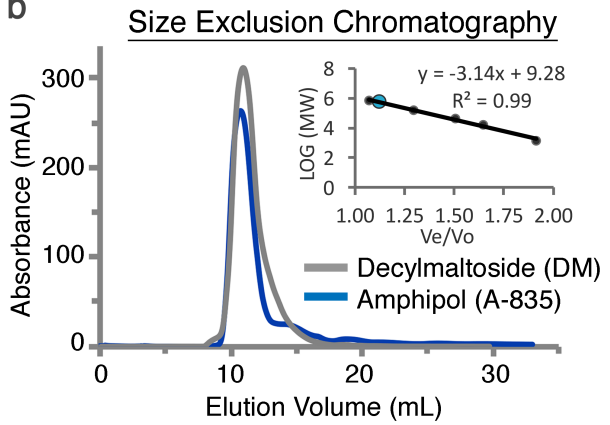

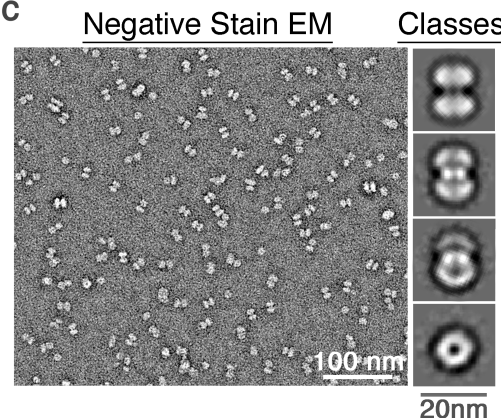

e

(i)

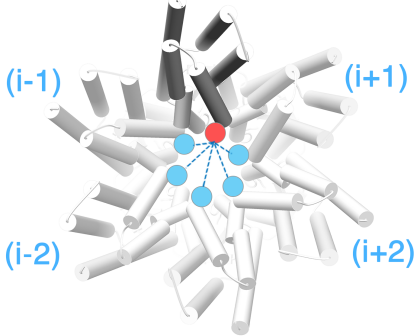

$(i+3)$

\begin{tabular}{|l|l|}
\hline Subunit-Subunit & Gly2 N-N Distance \\
\hline
\end{tabular}

\begin{tabular}{|c|c|}
\hline$(i)-(i \pm 1)$ & $9.1 \AA$ \\
\hline$(i)-(i \pm 2)$ & $15.8 \AA$ \\
\hline$(i)-(i \pm 3)$ & $18.2 \AA$ \\
\hline
\end{tabular}

(cross-linker length $=11.4 \AA$ )

f

\begin{tabular}{|l|l|l|l|l|l|l|}
\hline Protein 1 & AA position & Peptide & Protein 2 & AA position & Peptide & Cross-linker \\
\hline Cx46 & 115 & EEEPPKAAGPAEEHQDPAPVRDDR & Cx46 & 115 & EEPPKAAGPAEEHQDPAPVR & DSS \\
\hline Cx50 & 2 & GDWSFLGNILEEVNEHSTVIGR & Cx50 & 2 & GDWSFLGNILEEVNEHSTVIGR & DSS \\
\hline Cx50 & 2 & GDWSFLGNILEEVNEHSTVIGR & Cx46 & 2 & GDWSFLGR & DSS \\
\hline Cx46 & 2 & GDWSFLGR & Cx46 & 2 & GDWSFLGR & DSS \\
\hline Cx50 & 271 & AKGYQLLEEEK & Cx46 & 2 & GDWSFLGR & BS3 \\
\hline Cx46 & 2 & GDWSFLGR & Cx46 & 2 & GDWSFLGR & BS3 \\
\hline Cx46 & 2 & GDWSFLGR & Cx46 & 105 & MEEKRK & BS3 \\
\hline Cx46 & 2 & GDWSFLGR & Cx50 & 143 & SSSSSKGTK & BS3 \\
\hline
\end{tabular}

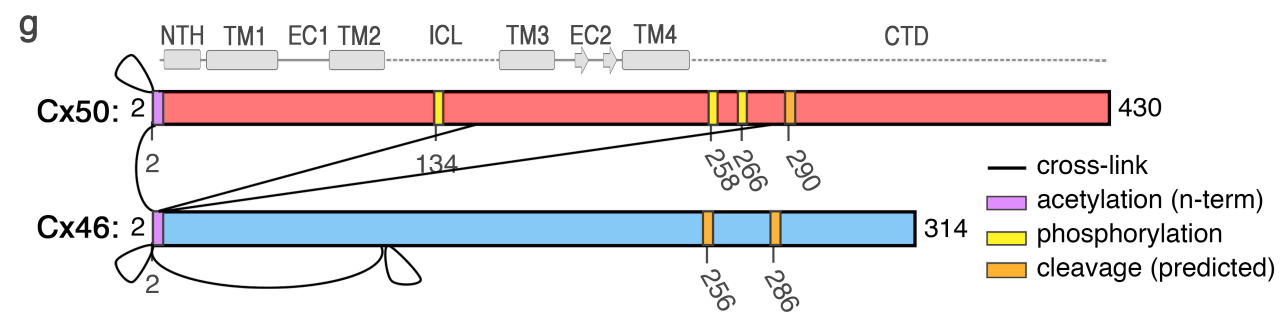


Extended Data Figure 1: Preliminary structural analysis of native lens Cx46/50 gap junction intercellular channels. (a) Denaturing protein gel and western-blot analysis of purified $\mathrm{Cx} 46 / 50$ isolated from lens core tissue (a.k.a. MP38). Protein bands corresponding to $\mathrm{C} \times 46$ and $\mathrm{C} \times 50$ co-migrate at a molecular weight of $\sim 38 \mathrm{kDa}$, consistent with age-dependent proteolytic cleavage within the c-terminal domain of both isoforms ${ }^{16}$. Performed 3 times with the similar results. (b) Size exclusion chromatography elution profile of Cx46/50 gap junctions reconstituted in decyl-maltoside (DM, grey trace) or amphipol (A-835, blue trace), monitored by UV-absorbance. Performed $>3$ times with similar results. Inset, shows calibration curve ( $\mathrm{n}=3$ runs) demonstrating Cx46/50 elutes at an apparent molecular weight of $\sim 560 \mathrm{kDa}$, consistent with the size of a dodecameric protein complex $(12 \times \sim 38 \mathrm{kDa})$ and two micelles $(2 x \sim 50 \mathrm{kDa})$. (c) EM micrograph of negatively stained Cx46/50 gap junctions reconstituted into amphipol. Scale bar $=100 \mathrm{~nm}$. (inset) Representative 2D class averages of negatively stained particles (selected from 25 classes). Scale bar $=20 \mathrm{~nm}$. (d-g) Chemical crosslinking and mass spectrometry. (d) Representative MS/MS m/z spectrum, identifying intersubunit cross-linking at the n-terminal Gly2 positions of Cx50 and Cx46. Identified peaks in the $\mathrm{m} / \mathrm{z}$ spectrum and amino acid identities are indicated $(\mathrm{Cx} 50 \mathrm{~b}$-ions = green; $\mathrm{Cx} 50$ y-ions = yellow; $\mathrm{Cx46}$ y-ions = blue $) . \mathrm{MS} / \mathrm{MS}$ data represent the consensus of 3 independent runs. (e) Structural analysis of cross-linking results, showing inter-subunit distances between the symmetrically related n-terminal Gly2 positions within the connexin hemichannel, ranging from $9.1 \AA$ ( $i$ to $i \pm 1$ ), $15.8 \AA$ ( $i$ to $i \pm 2$ ), and $18.2 \AA$ ( $i$ to $i \pm 3$ ). The cross-linker spacer length is $11.4 \AA$, indicating an (i to $i \pm 1$ ) arrangement of Cx50 and Cx46 within the same hemi-channel was most likely identified, although other arrangements may not be ruled out. (f) Overview of identified inter-subunit cross-links between $\mathrm{Cx} 50$ and $\mathrm{C} \times 46$ assembled gap junctions. Residues in red indicate the site of primary amines involved in the crosslinking reaction using either DSS or BS3. All detected inter-subunit crosslinks are between cytoplasmic domains. (g) Schematic showing sites of intersubunit cross-linking between $\mathrm{C} \times 46$ and $\mathrm{C} \times 50$ (black lines) and posttranslational modifications identified during proteomics analysis (yellow = phosphorylation; purple $=\mathrm{n}$-terminal acetylation). Met1 was determined to be removed in both Cx46 and Cx50 and the resulting n-terminal Gly2 position was identified in both acetylated and non-acetylated forms for Cx46 and Cx50, consistent with the specificity of the NatA acetylation complex ${ }^{24}$. Also shown are the predicted c-terminal domain (CTD) cleavage sites in Cx46 and Cx50 (orange), based on previous analysis of bovine Cx46/50 isolated from lens core tissue ${ }^{16}$. Secondary structure and domain labels are indicated for the nterminal helix (NTH), transmembrane helices (TM1-4) and extracellular domains (EC1-2) intracellular loop (ICL) and CTD. 

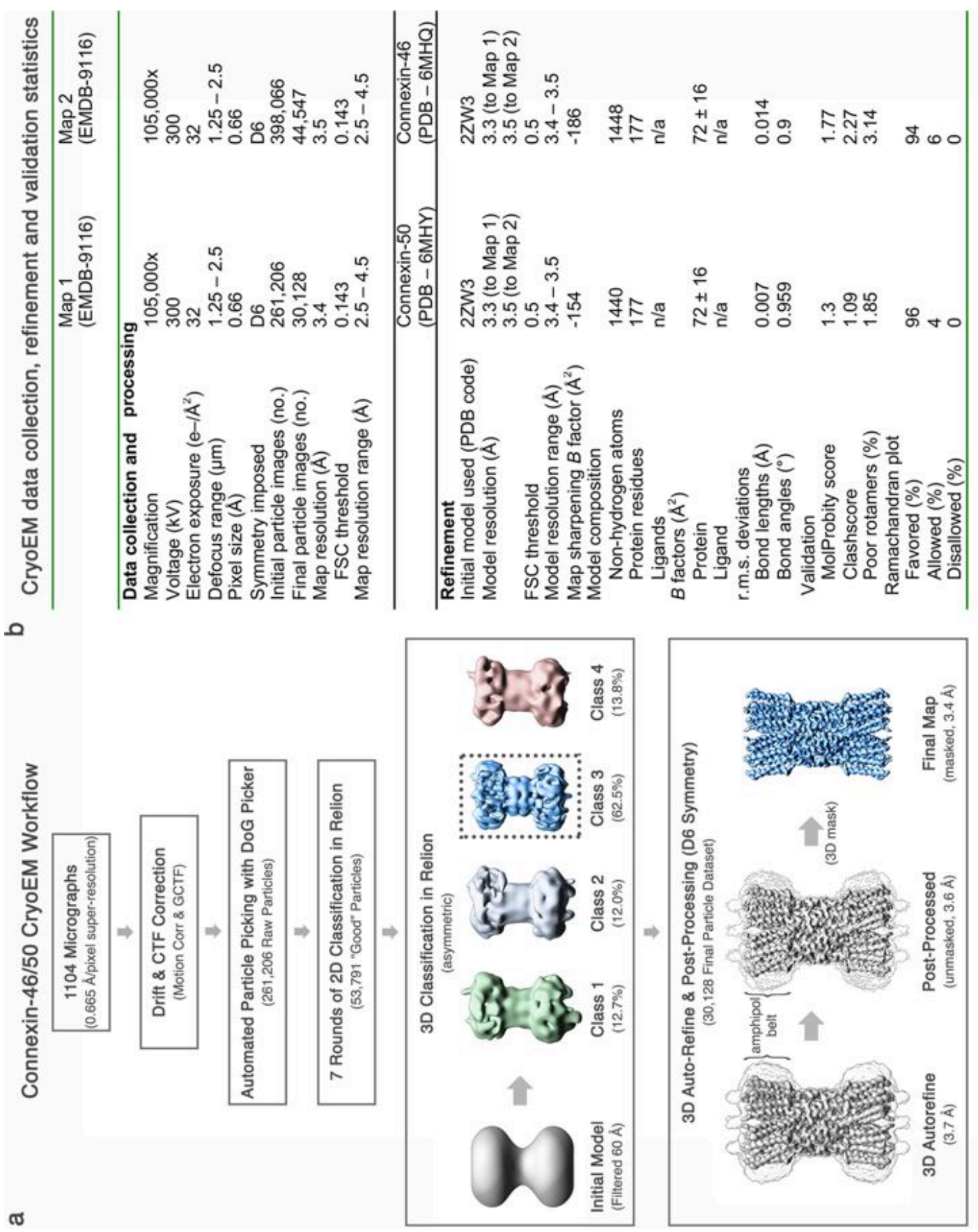
Extended Data Figure 2: Overview of CryoEM image processing and 3D reconstruction. (a) A total of 1104 micrographs were collected in an automated fashion using SerialEM ${ }^{65}$ on a $300 \mathrm{kV}$ Titan Krios (Dataset 1). Movie stacks were recorded using a K2 summit direct electron detector operated in super-resolution mode and acquired with an effective pixel size of $0.665 \AA$. Movie stacks were corrected for drift and contrast transfer function (CTF) using MotionCor $2^{66}$ and GCTF $^{67}$, respectively. An initial dataset of 261,206 raw particles was obtained using unbiased autopicking procedures in DoG Picker ${ }^{68}$. A refined dataset of 53,791 'good' particles was obtained following several rounds of $2 \mathrm{D}$ classification and removal of 'bad' particles (or ice contamination) in Relion ${ }^{69}$. Three-dimensional (3D) classification was seeded using an initial model obtained by negative stain EM, filtered to $60 \AA$. A majority of particles fell into a single 3D-class ( $62.5 \%$ of the "good" particles). These 30,128 particles were used for final 3D auto-refinement and post processing, yielding a final map at $3.4 \AA$ resolution by gold-standard FSC (Dataset 1). Dataset 2 was processed in a similar fashion from a total of 2,197 micrographs and 44,547 'good' particles, resulting in a final map at $3.5 \AA$ resolution by gold-standard FSC. (b) Summary of CryoEM data collection, refinement and model validation statistics. Dataset 1 was used to obtain the 3.4 A resolution reconstruction (Map 1). Dataset 2 was used to obtain the 3.5 $\AA$ resolution reconstruction (Map 2). Pre-processed and post-processed maps and associated masks from both datasets have been deposited to the EM databank (EMD-9116). The original multi-frame micrographs have been deposited to EMPIAR (EMPIAR-10212). Coordinates for Cx50 and Cx46 atomic models have been deposited to the Protein Data Bank (6MHY and $6 \mathrm{MHQ}$, respectively). 
a.

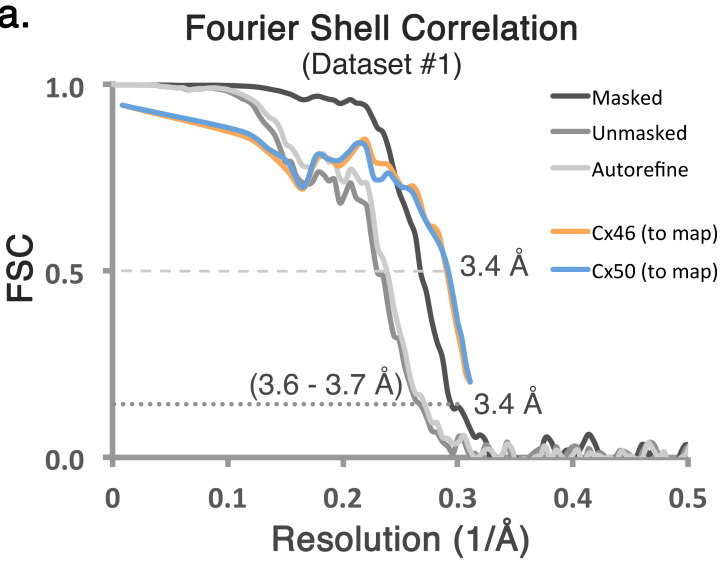

c.

Local Resolution (Dataset \#1)
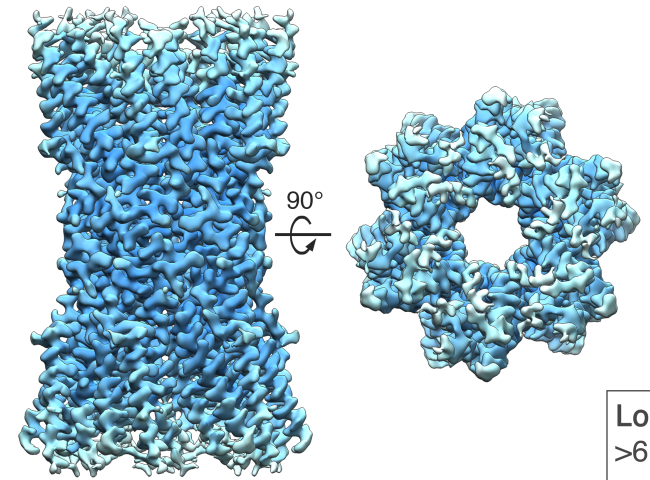

e.

Cx46 Model vs Map (Dataset \#1)

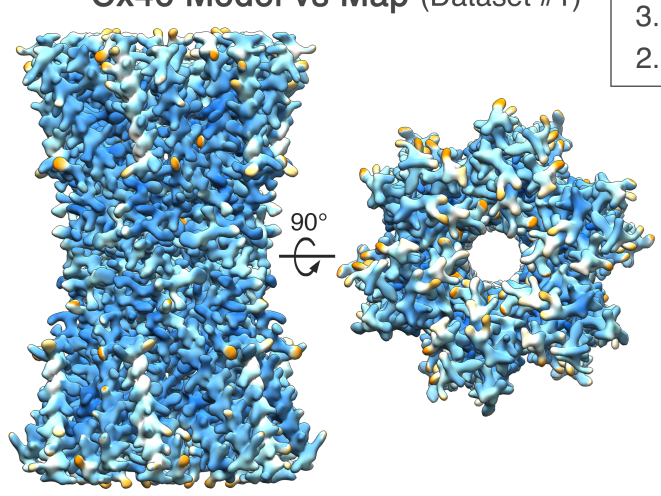

b. Fourier Shell Correlation

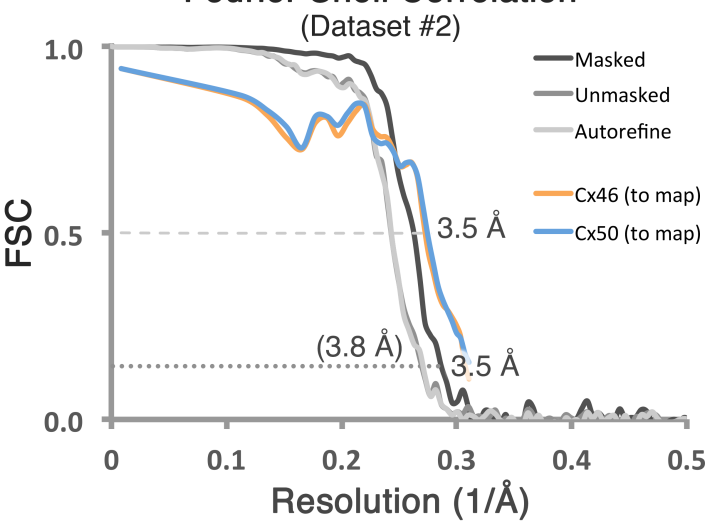

d.

Local Resolution (Dataset \#2)

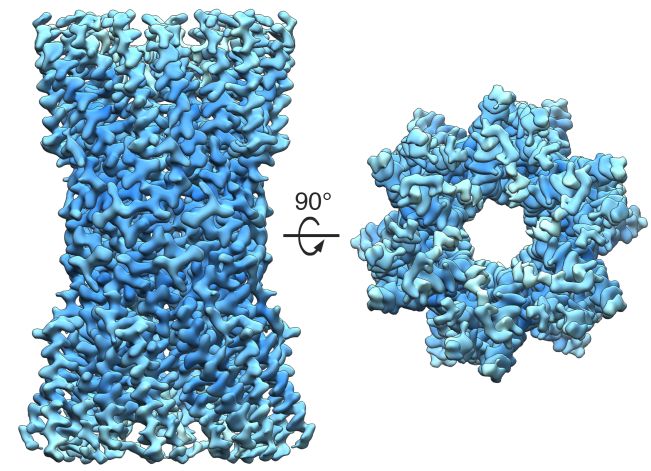

f. Cx50 Model vs Map (Dataset \#1)

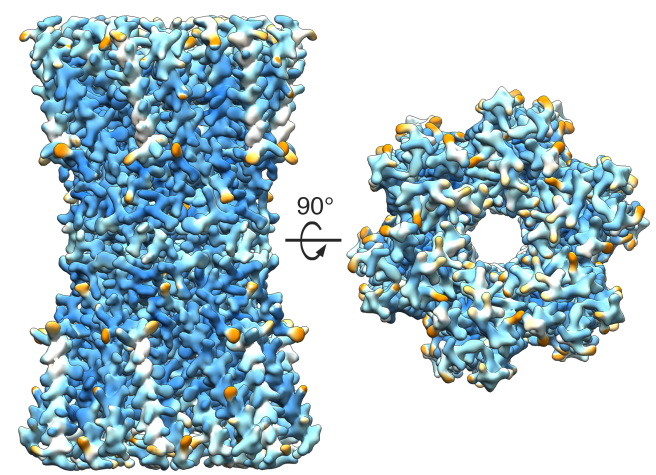


Extended Data Figure 3: Global and local resolution analysis. (a,b) Fourier Shell Correlation (FSC) analysis obtained from Dataset 1 and Dataset 2, respectively. Gold-standard FSC curves following auto-refinement (light grey), post-processing (grey), and masking (dark grey). The final masked maps display an overall resolution of $\sim 3.4 \AA$ (Dataset 1 ) and $\sim 3.5 \AA$ (Dataset 2), using a 0.143 cut-off. FSC curves comparing atomic models of Cx46 (orange) and $\mathrm{Cx} 50$ (blue) fit to the CryoEM maps display correlation at 0.5 cutoff to a resolution of $3.4 \AA$ (Dataset 1) and $3.5 \AA$ (Dataset 2). (c,d) Local resolution analysis using BlocRes ${ }^{70}$, obtained for the half-maps for Dataset 1 and 2, respectively. (e,f) Local resolution analysis comparing the experimental density map (Dataset 1) to the calculated maps of Cx46 and Cx50, respectively. Local resolution ranges in panels $c-f$ are indicated by color $(2.5-$ $4.0 \AA$ = blue - cyan; $4.0-5.0 \AA$ = white; $5.0-6.5 \AA$ yellow - orange). Tabulated values obtained for local resolution of $\mathrm{C} \times 46$ and $\mathrm{Cx} 50$ models compared to the experimental density map are provided (Supplementary Tables 2,3). Local resolution assessment comparing the density map to the two models indicates that the sites where the two isoforms differ in sequence were generally less well resolved as compared to equivalently exposed residues where $\mathrm{C} \times 46$ and $\mathrm{Cx} 50$ are conserved in sequence. 


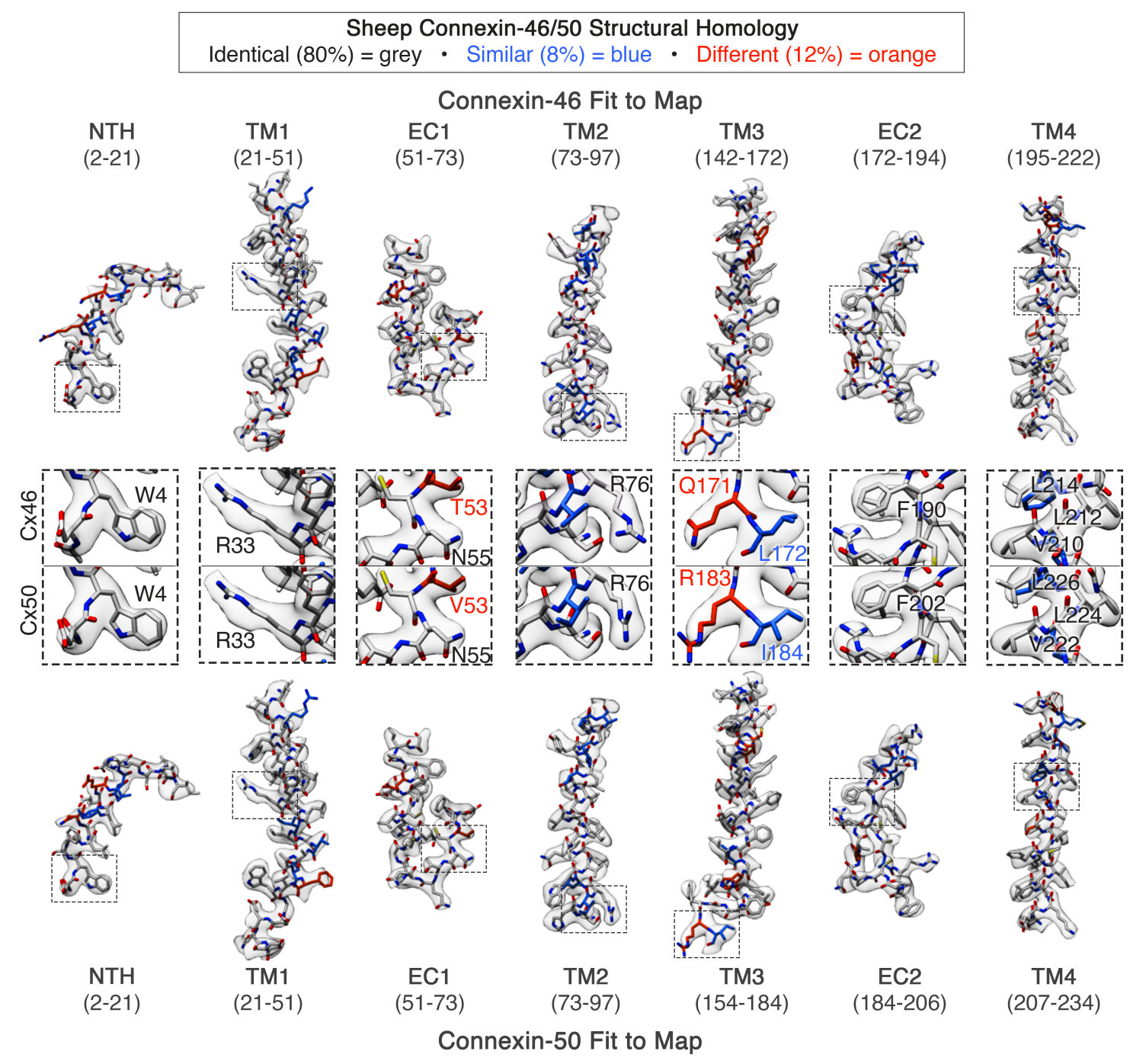


Extended Data Figure 4: Connexin-46 and -50 atomic models fit to the CryoEM density maps. Segmented CryoEM map with atomic models for sheep Connexin-46 (Cx46) and Connexin-50 (Cx50) fit to the experimental densities derived from Dataset 1 (3.4 A, D6-symmetry), including regions for transmembrane domains 1-4 (TM1-4) and extracellular domains 1-2 (EC1-2). The n-terminal helix domain (NTH) is fit into the map from Dataset 2 (3.5 $\AA$, D6-symmetry), which was more well defined in this region. Cx46 (top) and Cx50 (bottom) models are colored according to their pair-wise sequence homology, as being identical (grey, 80\%), similar (blue, $8 \%$ ) and different (orange, 12\%). Windows show zoom-views corresponding to boxed regions of the segmented maps, highlighting representative sidechain densities and fit to the atomic models. Regions of identical or similar amino acids are fit equally well by both models (e.g., Cx46 L172 vs. Cx50 I184, blue labels). Over regions where the sequence of $\mathrm{C} \times 46$ and $\mathrm{Cx} 50$ differ, sidechain density is typically weaker (see also, Extended Data Fig. 5). This is possibly due to the imposed averaging of two different sidechains in these areas, or relative flexibility as many of these residues correspond to solvent exposed sidechains. In these areas of difference, and where EM density is present, both Cx46 and Cx50 models were typically fit equally well into the density map (e.g., Cx46 T53 vs. Cx50 V53 and Cx46 Q171 vs. Cx50 R183, orange labels). 


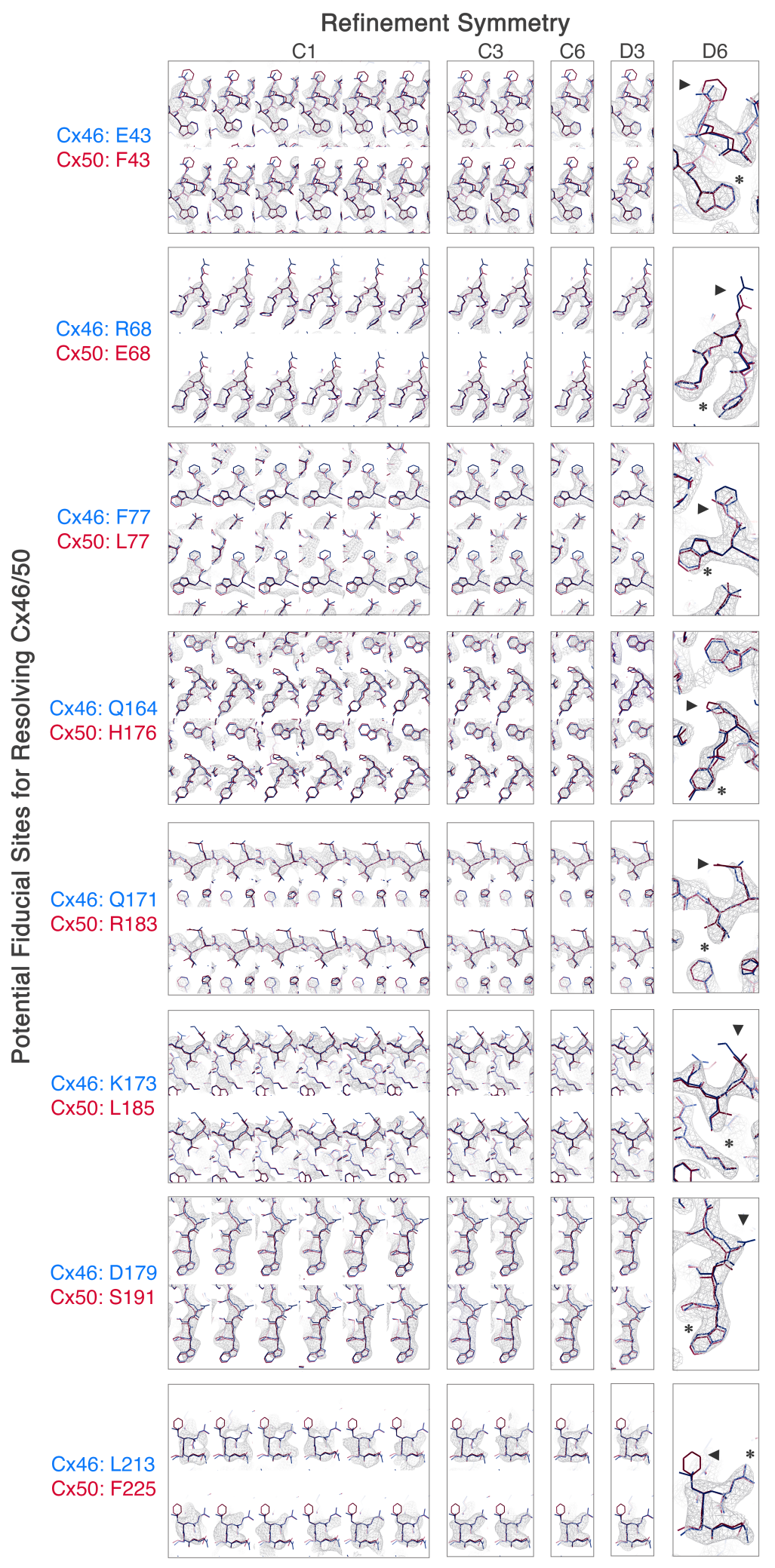


Extended Data Figure 5: Analysis of different symmetry refinements on the ability to resolve differences between Cx46 and Cx50. Eight sites of sequence differences involving bulky amino acids (labeled, and indicated by arrow head) were selected as potential fiducial markers for resolving the two different isoforms following 3D refinement with various applied symmetries (C1: $4.1 \AA$ resolution; C3: $3.9 \AA$ resolution, C6: $3.7 \AA$ resolution, D3: $3.7 \AA$ resolution; D6: $3.4 \AA$ resolution). For the applied symmetries, views are presented for each unique asymmetric subunit (boxed). Despite the modest resolution of the asymmetric (C1) reconstruction, sidechain density for bulky amino acids were typically observed at sites where the two isoforms are conserved (asterisk). However, at the selected sites of sequence variation (arrow head) the sidechain densities are either not well resolved, or there was no systematic variation that indicated an ability to distinguish the two isoforms. The most resolved features at these sites of variation were obtained with D6 symmetry, and typically corresponded to regions where these different amino acids share similar structure (e.g., Cb positions). Although the CryoEM density at these sites of variation were typically weak, the resolvable sidechain features throughout the rest of the map were generally enhanced when higher symmetry was applied during map refinement (indicated by asterisk), suggestion that regions of sequence similarity between $\mathrm{Cx} 46 / 50$ also share a high level of structural similarity. 


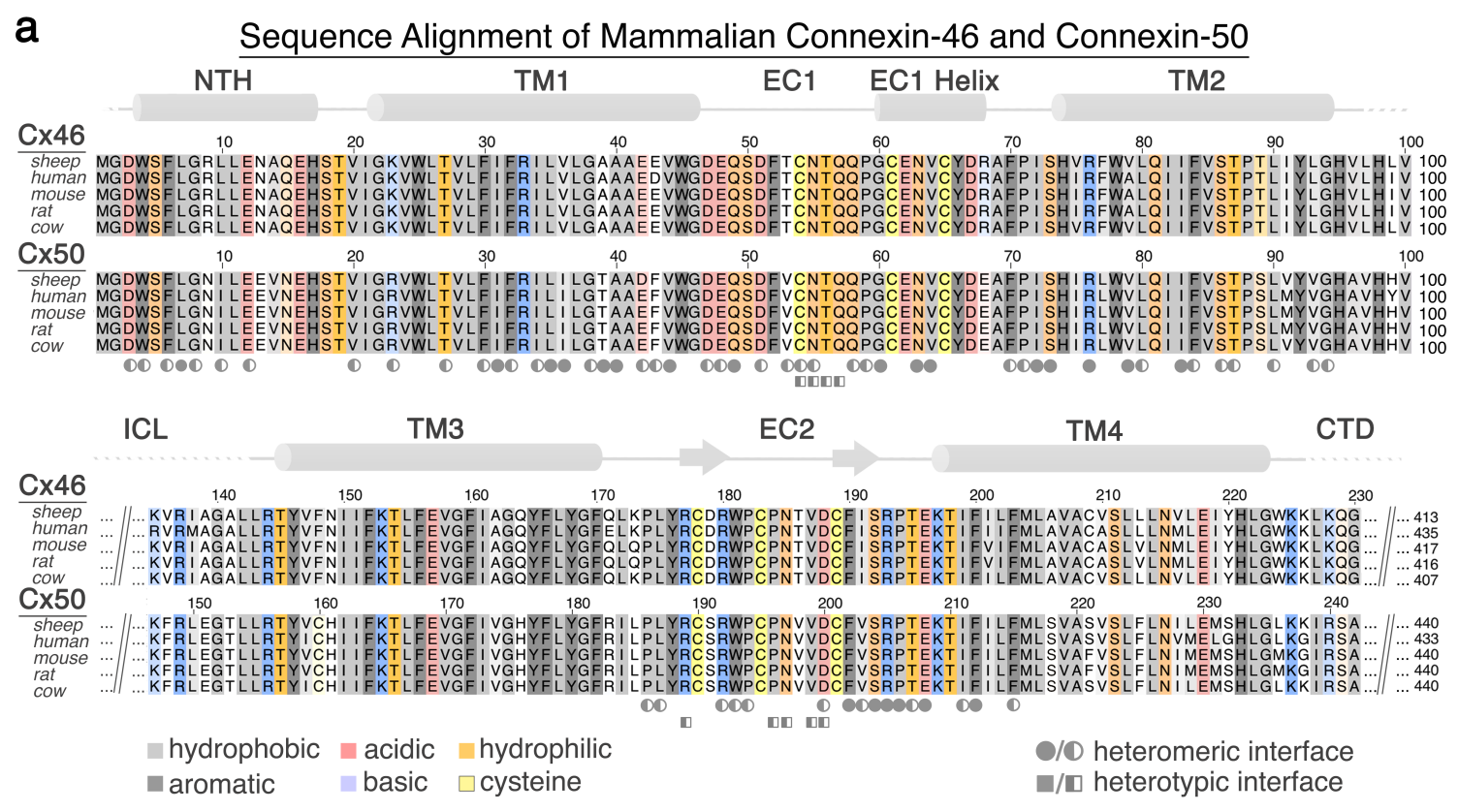

b Variable Sites of Heteromeric Interface Interactions

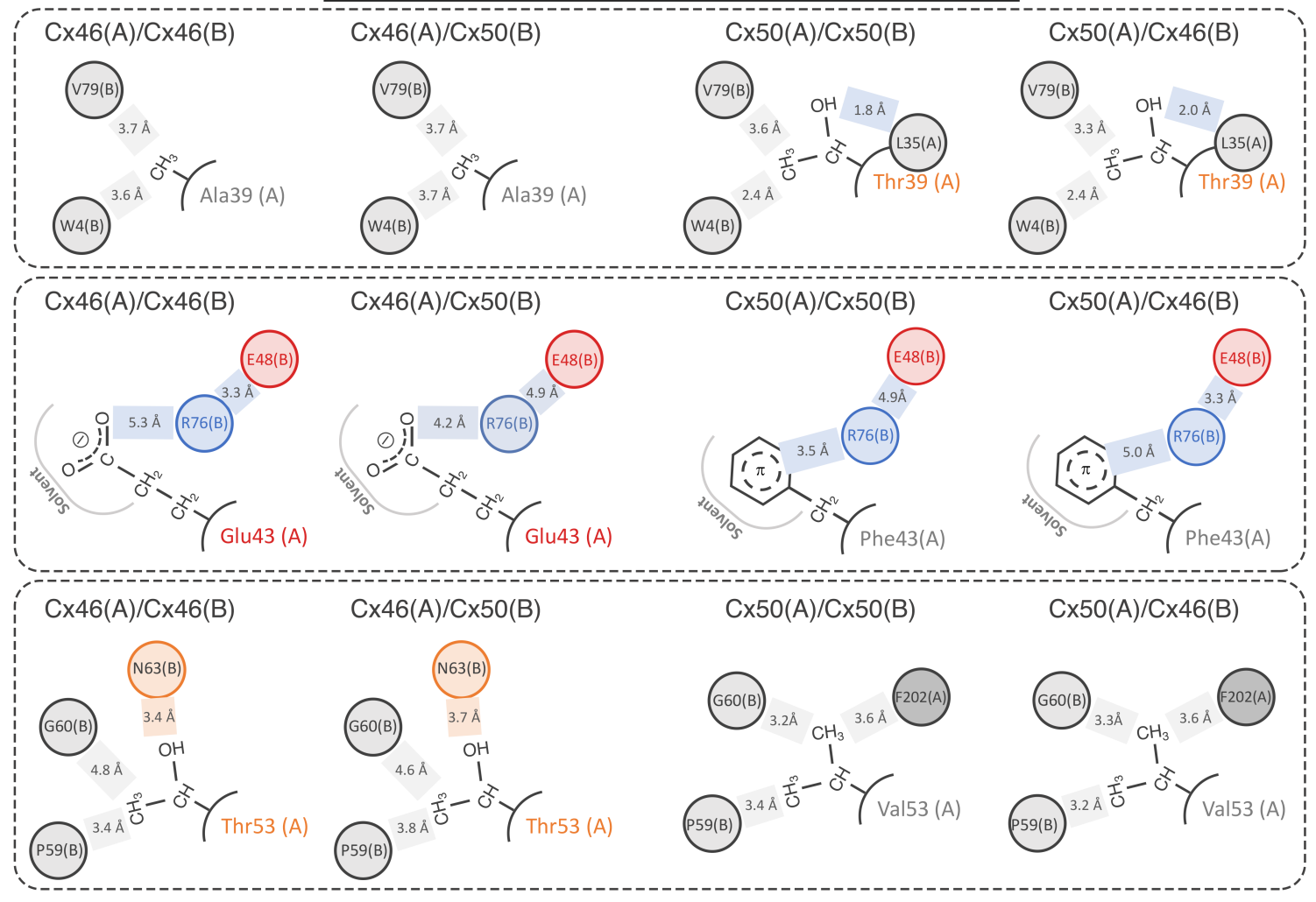


Extended Data Figure 6: Sequence and structure conservation of Cx46 and Cx50 heteromeric/heterotypic interfaces (a) Multiple sequence alignment of mammalian Cx46 and Cx50 isoforms with residues contributing to heteromeric and heterotypic interfaces annotated (circle - heteromeric interface, square - heterotypic interface; filled $=>70 \%$ buried and half-filled $=$ $20-70 \%$ burial) ${ }^{83}$. Coloring corresponds to amino acid type (grey hydrophobic; dark grey - aromatic; red - acidic; blue - basic; orange hydrophilic; yellow - cysteine). Regions of sequence homology are indicated by the level of shading. Secondary structure and domain labels are indicated for the n-terminal helix (NTH), transmembrane helices (TM1-4) and extracellular domains (EC1-2). Regions lacking defined structure and of poor sequence homology within the intracellular loop (ICL) and c-terminal domain (CTD) have been omitted for clarity. Sheep and human Cx46 and Cx50 orthologs contain $\sim 95 \%$ sequence identity ( $98 \%$ similarity) over the structured regions of the protein. Numbering corresponds to the amino acid sequence of sheep Cx44 and Cx49 used in the main text. (b) Illustration of homomeric and heteromeric interface interactions involving the 3 sites lacking conservation between Cx44 and Cx49 at this interface (positions 39, 43 and 53). Despite these sequence differences, the interactions involving these residues are generally similar (hydrophobic - grey; h-bonding - orange; and ion-pairing - blue). 
a

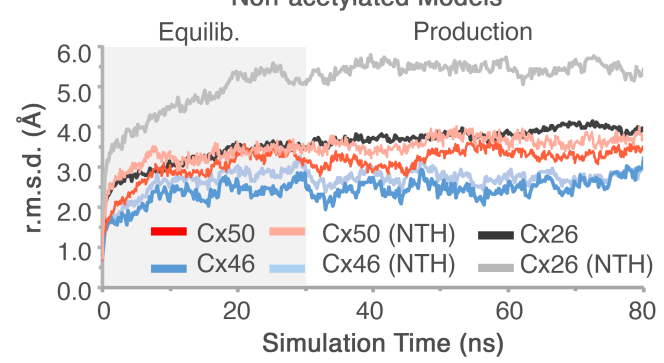

b

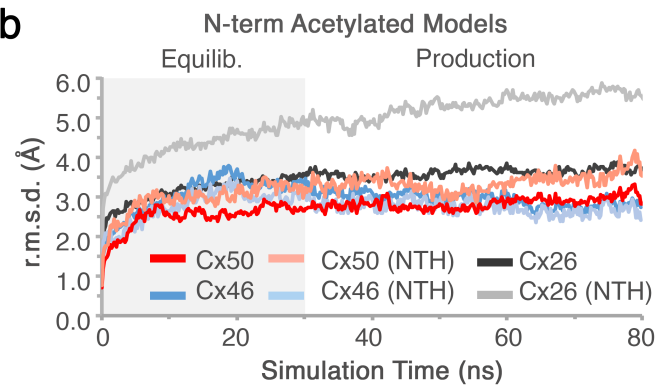

C
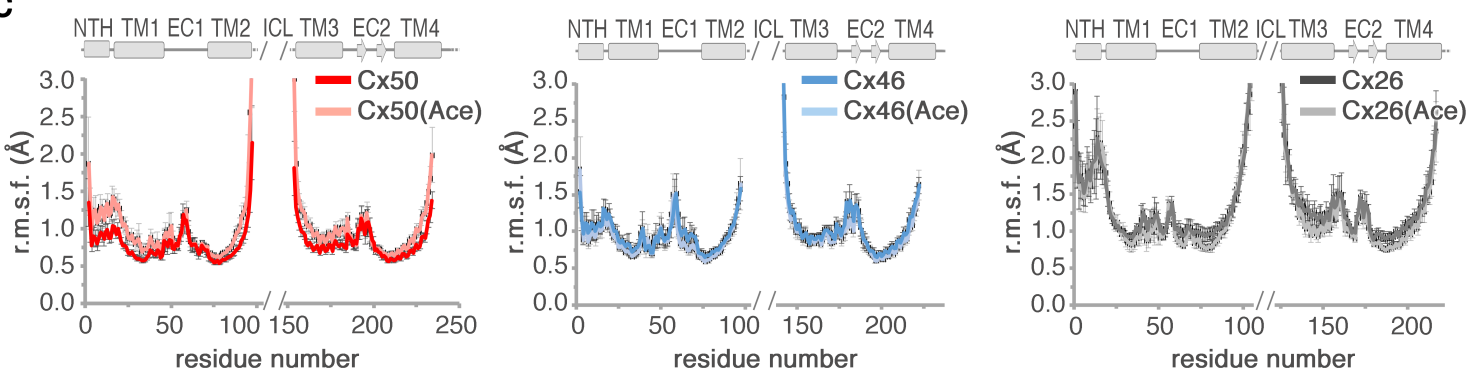

\section{d}
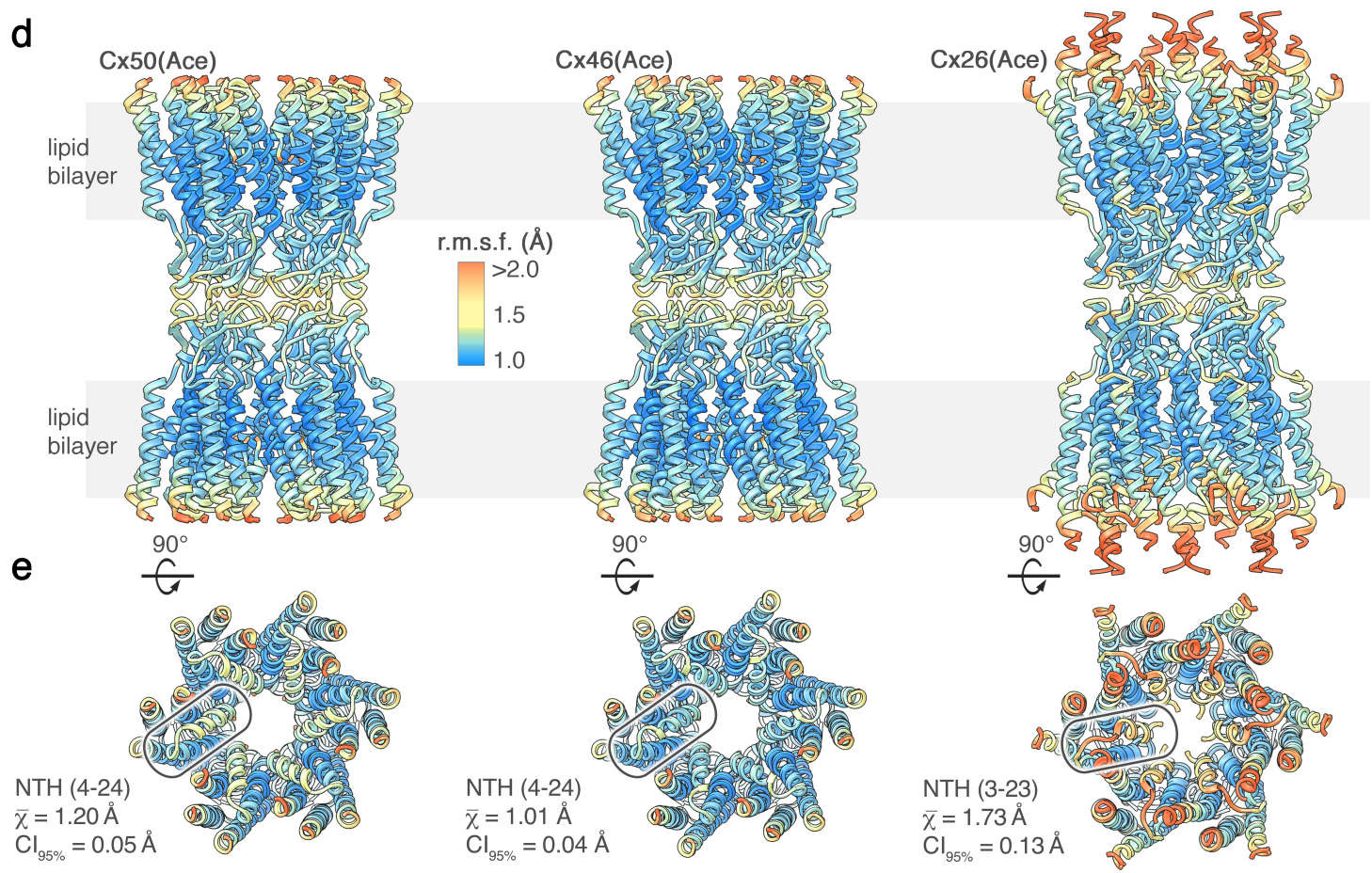
Extended Data Figure 7: Analysis of protein backbone dynamics during MD equilibration and production. (a) $\mathrm{C} \alpha$ root mean squared deviation (r.m.s.d.) analysis of equilibrium ( $0-30 \mathrm{~ns})$ and production phases (30-80 ns) of the molecular dynamics (MD) simulations, calculated with respect to the experimental starting structure for non-acetylated models of $\mathrm{Cx} 50$ (red traces) and Cx46 (blue traces) and Cx26 (with Met1 added) (grey traces). Separate analysis for the $n$-terminal helix (NTH) domains are shown in lighter shades. (b) Same analysis as in panel a, for models with n-terminal acetylation added. The NTH domain of Cx26 (light grey traces) shows significantly higher r.m.s.d. values, for both non-acetylated and acetylated models. (c) Plot of average $\mathrm{C} \alpha$ root mean squared fluctuation (r.m.s.f.) during the production phase of the molecular dynamics (MD) simulations for Cx50 (left, red traces), Cx46 (center, blue traces) and Cx26 (right, grey traces). Data obtained for the n-terminal acetylated models are shown in lighter shades. Averages are determined for the 12 subunits composing the intercellular channel. Error bars represent $95 \%$ confidence intervals ( $n=12$ subunits). Secondary structure and domain labels are indicated for the n-terminal helix (NTH), transmembrane helices (TM1-4), extracellular domains (EC1-2) and intracellular loop (ICL; not modeled). (d,e) Average r.m.s.f. values of the acetylated models mapped to the experimental starting structures of Cx50 (left), Cx46 (center) and Cx26 (right). Colors correspond to r.m.s.f. amplitudes: $0-1.0 \AA$ (cyan), $1.0-2.0 \AA$ (yelloworange), > $2.0 \AA$ (red). In panel e, a single NTH domain is circled and the average r.m.s.f. values and $95 \%$ confidence intervals (student t-test) calculated over the NTH domain of each isoform are displayed $(n=12$ subunits). The NTH domain of Cx26 shows significantly higher r.m.s.f. values, for both acetylated and non-acetylated models $(P<0.0001)$. 


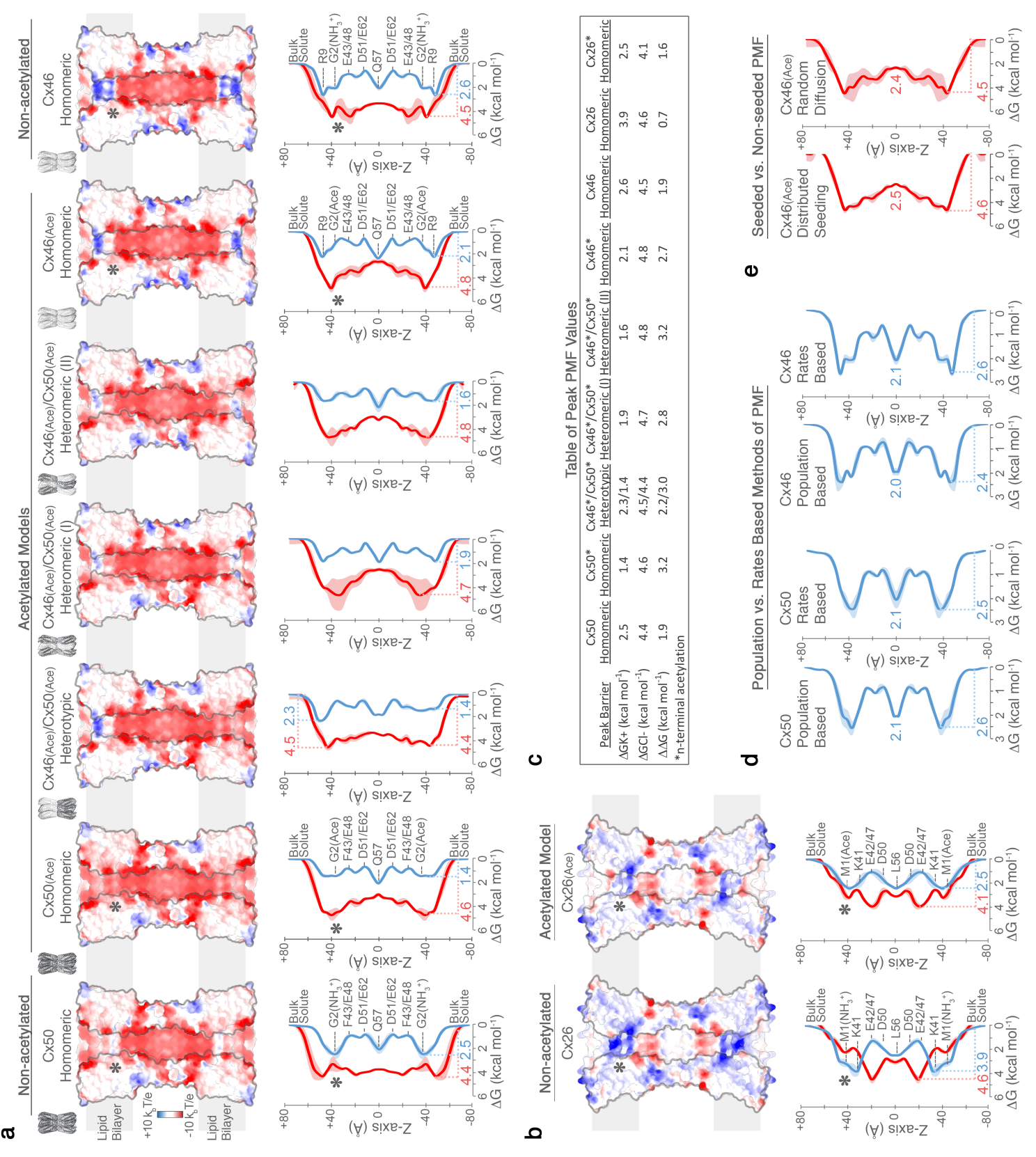


Extended Data Figure 8: Modulation of coulombic surface potential and $\mathrm{K}^{+} / \mathrm{Cl}^{-}$PMFs resulting from $n$-terminal acetylation and $\mathrm{Cx46/50}$ heterotypic/heteromeric assembly. (a) Coulombic surface potential maps (top) and potential-of-mean-force (PMF, $\Delta \mathrm{G} \mathrm{kcal} \mathrm{mol}^{-1}$ ) (bottom) obtained for a set of non-acetylated and acetylated $\mathrm{Cx} 50 / \mathrm{Cx} 50_{(\text {Ace) }}$ and $\mathrm{Cx} 46 / \mathrm{C} \times 46_{(\mathrm{Ace})}$ models characterized by MD simulation. Acetylated $\mathrm{Cx} 50_{\text {(Ace) }}$ and $\mathrm{Cx} 46_{\text {(Ace) }}$ monomers were used to construct a heterotypic channel and two different heteromeric channels (labeled, I and II). Icons at the top of each structure show the relative configurations of $\mathrm{Cx} 50$ (black) and $\mathrm{Cx} 46$ (white). The resulting coulombic surface potentials are colored as in Fig. 3a (negative red, neutral - white and positive - blue). Only 8 subunits are shown to portray both the channel pore and subunit interfaces. An asterisk (panels $a-b$ ) indicates the site of $n$-terminal acetylation, which neutralizes the positively charged n-terminus. PMFs obtained for $\mathrm{K}^{+}$(blue traces) and $\mathrm{Cl}^{-}$ions (red traces) are displayed directly beneath each model displayed in panel a. Free energy maxima are labeled and pore axis (z-axis) is indicated. Traces indicate symmetrized values, with unsymmetrized values in lighter shading. In the case of the asymmetric Cx46/50 heterotypic model (middle left), PMFs represent the average from the first and last $\sim 60 \mathrm{~ns}$ of simulation. (b) Coulombic surface potential (top) and PMFs (bottom) obtained for non-acetylated (left) and acetylated (right) $\mathrm{C} \times 26 / \mathrm{C} \times 26$ (Ace) are displayed as in panel a. (c) Table of peak free energy barriers for $\mathrm{K}^{+}\left(\Delta \mathrm{G}_{\mathrm{K}+}\right)$ and $\mathrm{Cl}^{-}\left(\Delta \mathrm{G}_{\mathrm{Cl}}\right)$ and corresponding $\Delta \Delta \mathrm{G}$, reported as a proxy for charge selectivity. Asterisk indicates models with $\mathrm{n}$ terminus acetylated. (d,e) Validation of methods used to construct the PMFs. (d) Comparison of $\mathrm{K}^{+}$PMFs obtained for $\mathrm{Cx} 50_{(\text {(Ace) }}$ and $\mathrm{C} \times 46_{(\text {(Ace) }}$ using population states (left) or transition rates (right) (see Methods). Both methods yielded similar PMF profiles. All other PMFs were constructed using the transition rates method. (e) Comparison of $\mathrm{Cl}^{-}$PMFs obtained for $\mathrm{Cx} 46_{(\text {Ace) }}$ using transition rates of $\mathrm{Cl}^{-}$ions that diffused into the pore (left) and those that were randomly seeded within the pore (right). All other $\mathrm{Cl}^{-}$PMFs were constructed using the distributed seeding approach to enhance sampling (see Methods). 

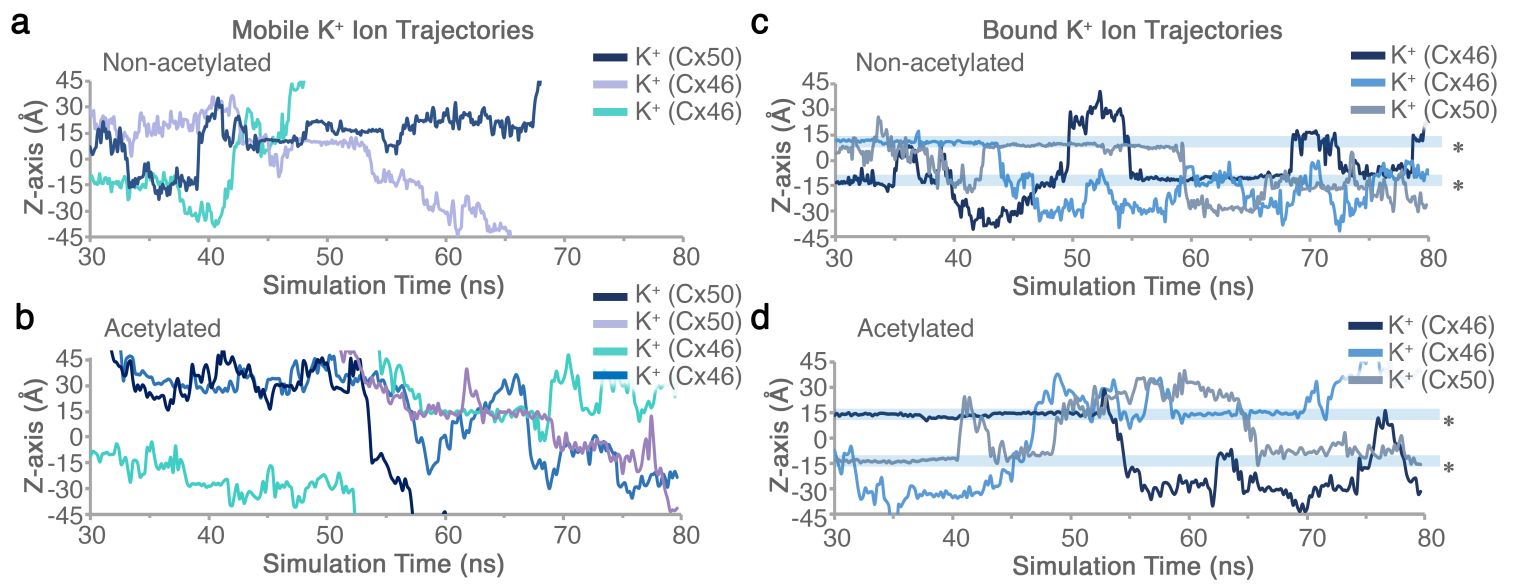

e

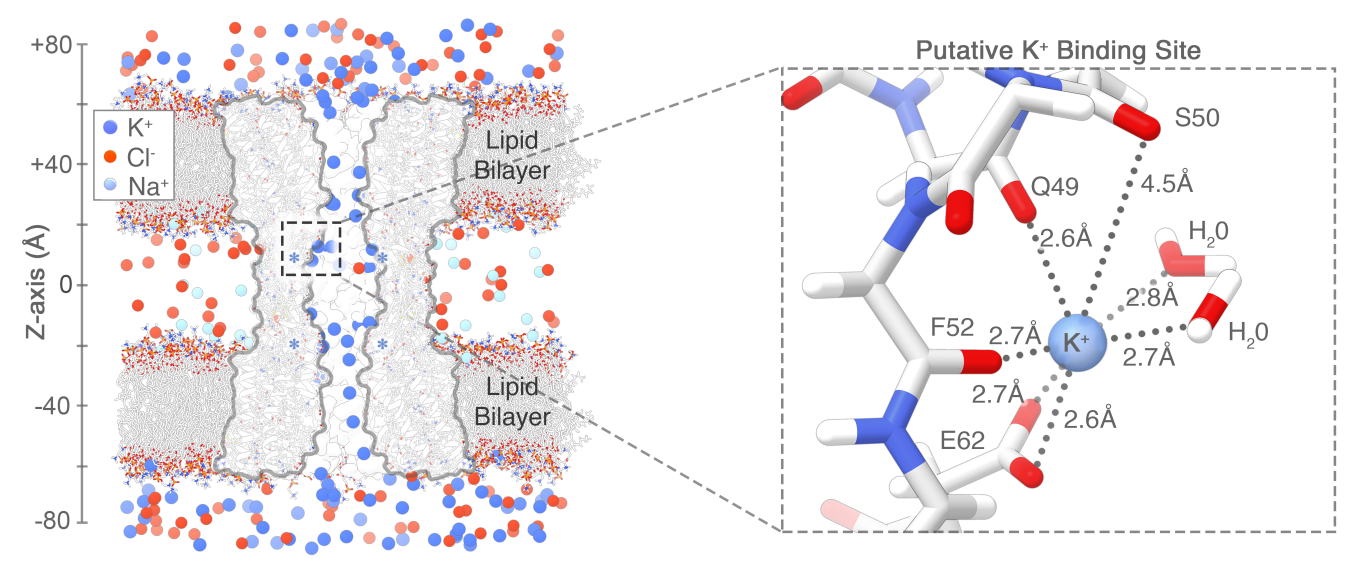


Extended Data Figure 9: Analysis of $\mathrm{K}^{+}$trajectories and putative binding site observed during MD simulation. (a-d) $\mathrm{K}^{+}$ion trajectories obtained for Cx50 and Cx46 along the channel pore (z-axis). (a,b) Representative traces of mobile ions transiting and exiting/entering the channel pore in both acetylated (panel a) and non-acetylated (panel b) models of Cx46 and Cx50. (c,d) $\mathrm{K}^{+}$ ions displaying long dwell times $(\sim 10-20 \mathrm{~ns})$ localized at a putative binding site(s) within the channel pore (asterisk at $\sim 14 \AA$, z-axis) in both models of Cx46 and Cx50. (a-d) Similar results were observed from 6 independent runs using non-acetylated models ( 1 x 80ns and $2 \times 10$ ns runs for both Cx50 and Cx46) and 13 independent runs using the acetylated models $(1 \times 80$ ns and 6 $x$ 10ns runs for Cx50; and $1 \times 80$ ns and $7 \times 10$ ns runs for Cx46). (e) Representative snapshot showing a zoom view of the putative $\mathrm{K}^{+}$binding site identified for $\mathrm{C} \times 50$ and $\mathrm{C} \times 46$, corresponding to the asterisked region in panels $c$ and $\mathrm{d}$. A single $\mathrm{K}^{+}$ion is bound by a conserved set of amino acids (amongst Cx46/50 orthologs), coordinated by the sidechain carboxylate of Glu62 and backbone carbonyls of Gln49, Ser50 and Phe52 (identical in Cx46 and Cx50). Two transient water molecules observed coordinating the bond $\mathrm{K}^{+}$ion are shown. A total of twelve binding sites are present within the dodecameric channel. Similar behavior was observed from simulations using both nonacetylated and acetylated models (a total of 19 independent simulations). A functional role for this putative binding site is not yet clear, but may represent a physiologically relevant cation binding site similar to the recently proposed $\mathrm{Ca}^{2+}$-binding site in $\mathrm{C} \times 26^{11}$. 

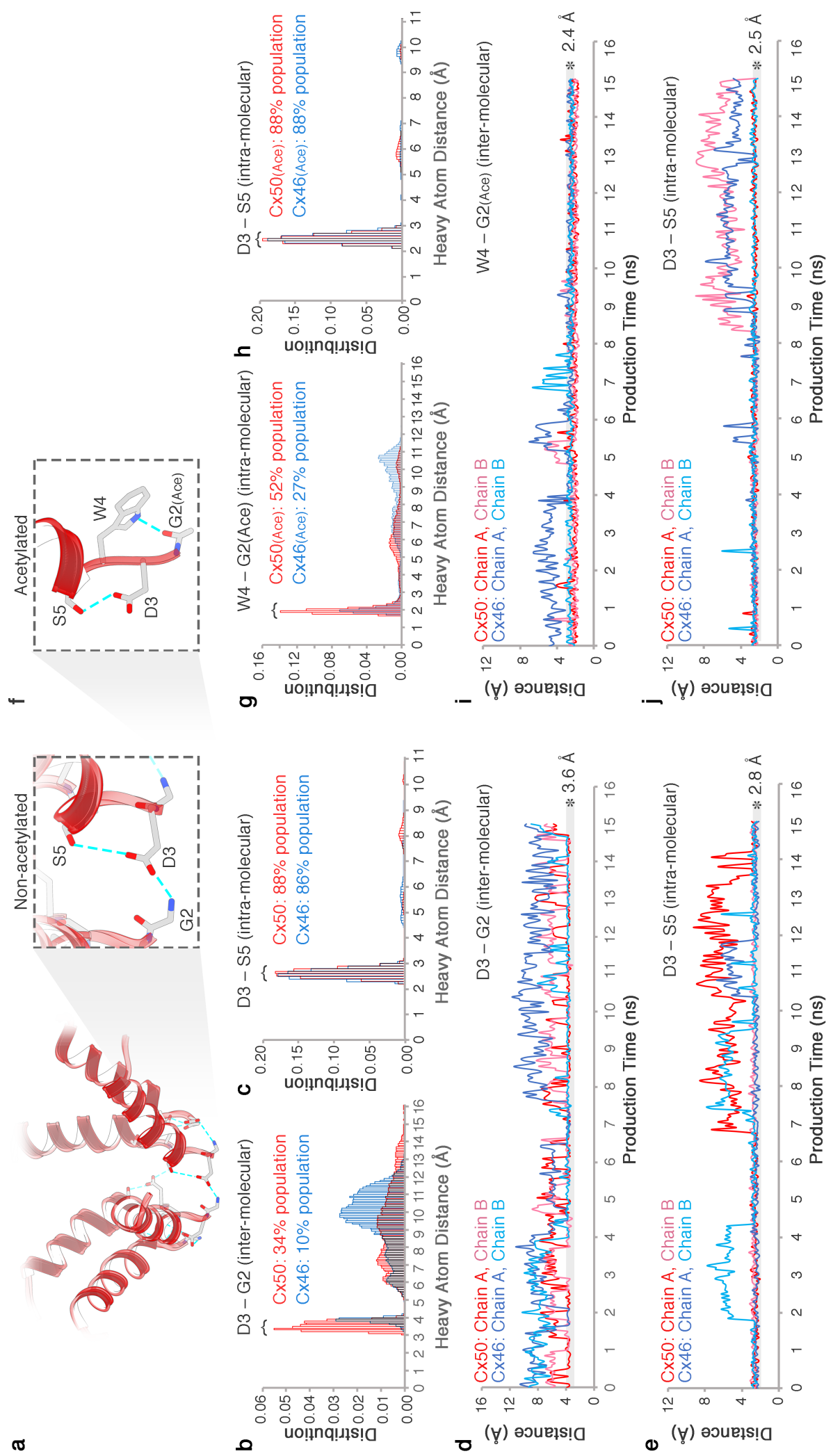
Extended Data Figure 10: Dynamic hydrogen bond network within the NTH domain observed by MD simulation. (a-e) Analysis of hydrogen bond interactions for non-acetylated models of Cx46 and Cx50 observed during MD simulation. (a) Inset, shows a zoom-view of D3 pairing with the positively charged n-terminal G2 position from a neighboring subunit (inter-molecular) and with the hydroxyl of S5 within the same subunit (intra-molecular). The D3G2 interactions are dynamically formed and broken during MD simulation, while the intra-molecular D3-S5 h-bond is relatively stable (as shown in panels b-e). (b) Population statistics of inter-atomic distances involving D3 (C $\gamma)$ and $\mathrm{G}(\mathrm{N})$ of the neighboring chain and (c) Ser5 $(\mathrm{H} \gamma)$ of the same chain, extracted from MD simulation production runs of Cx46 (blue histogram) and Cx50 (red histogram). For D3 and G2, heavy atoms were chosen as proxies to monitor hydrogen bonding interactions involving equivalent rotameric donor-acceptor configurations. The population centered at $\sim 3.6 \AA$ (panel b) and $\sim 2.8 \AA$ (panel c) are considered to be within hydrogen bond distance. (d) Trajectories extracted from MD simulation of Cx46 (blue traces) and Cx50 (red traces) showing the dynamical behavior of the D3-G2 inter-molecular charge paring and (e) D3-S5 intra-molecular h-bonding. The dwell times showing hydrogen bond pairing ( $\sim 3.6 \AA$ in panel $\mathrm{d}$; and $\sim 2.8 \AA$ in panel e) are indicated with transparent grey shading. In the Cx26 crystal structure, the equivalent D2 site is modeled in hydrogen bond distance to a neighboring T5 site (Cx26 numbering $)^{10}$, but this inter-molecular interaction is rapidly broken during MD simulations and does not appear to re-from within the time-scale of our MD experiments, and instead forms a stable intra-molecular interaction with T5, as described previously ${ }^{35}$ (data not shown). (f-j) Analysis of hydrogen bond interactions observed during MD simulation for $\mathrm{Cx} 46_{(\text {Ace) }}$ and $\mathrm{Cx} 50_{\text {(Ace) }}$ modeled with the n-terminal G2 position acetylated. (f) Inset, shows a zoomview of acetylated G2(Ace) position h-bonded to the indole ring of W4 from the same subunit (intra-molecular) and the same intra-molecular D3-S5 h-bond interaction observed in the non-acetylated channel. (g) Population statistics of inter-atomic distances involving $\mathrm{W} 4(\mathrm{~N} \varepsilon)$ and G2(Ace) (acetyl-carbonyl) and (h) and D3 $(\mathrm{C} \gamma)$ distance to Ser5 $(\mathrm{H} \gamma)$ of the same chain, extracted from MD simulation production runs of $\mathrm{Cx} 46_{\text {(Ace) }}$ (blue histogram) and $\mathrm{Cx50}$ (Ace) (red histogram). (i) Trajectories extracted from MD simulation of $\mathrm{Cx} 46_{(\mathrm{Ace})}$ (blue traces) and $\mathrm{Cx} 50_{\text {(Ace) }}$ (red traces) showing the dynamical behavior of the W4G2(Ace) h-bond paring and (j) D3-S5 intra-molecular h-bonding. The dwell times showing hydrogen bond pairing $(\sim 2.4 \AA$ in panel $\mathrm{i}$; and $\sim 2.5 \AA$ in panel j are indicated with transparent grey shading. For clarity, only the first $15 \mathrm{~ns}$ of the production period is shown (panels $d-e$ and $i-j$ ). Similar results were observed from 6 independent runs using non-acetylated models $(1 \times 80 \mathrm{~ns}$ and $2 \times 10$ ns runs for both $\mathrm{C} \times 50$ and $\mathrm{C} \times 46$ ) and 13 independent runs using the acetylated models ( $1 \times 80$ ns and $6 \times 10$ ns runs for Cx50; and $1 \times 80 \mathrm{~ns}$ and 7 $x 10$ ns runs for $\mathrm{Cx} 46)$. 


\begin{tabular}{|c|c|c|c|c|c|c|}
\hline & Connexin-50 & Connexin-50* & Connexin-46 & Connexin-46* & Connexin-26 & Connexin-26* \\
\hline Total Atoms & 364,952 & 363,722 & 360,190 & 355,524 & 368,756 & 367,346 \\
\hline Solvent & 196,248 & 194,928 & 191,346 & 191,310 & 194,856 & 193,548 \\
\hline Lipids & 133,866 & 133,866 & 133,732 & 129,042 & 133,464 & 133,330 \\
\hline Protein & 34,440 & 34,488 & 34,716 & 34,764 & 39,972 & 40,020 \\
\hline Ions & 398 & 440 & 396 & 408 & 464 & 448 \\
\hline Modelled Residues & $2-97 ; 154-234$ & $2-97 ; 154-234$ & $2-97 ; 142-222$ & $2-97 ; 142-222$ & $1-109 ; 125-217$ & $1-109 ; 125-217$ \\
\hline \multicolumn{7}{|l|}{ Simulation Conditions } \\
\hline Simulation Box $(\AA)$ & $152 \times 152 \times 176$ & $152 \times 152 \times 176$ & $152 \times 152 \times 176$ & $152 \times 152 \times 176$ & $155 \times 155 \times 176$ & $155 \times 155 \times 176$ \\
\hline Pressure (atm) & 1 & 1 & 1 & 1 & 1 & 1 \\
\hline Temperature (K) & 310 & 310 & 310 & 310 & 310 & 310 \\
\hline Time Step (fs) & 1 & 2 & 1 & 2 & 2 & 2 \\
\hline Equilibration Time (ns) & 30 & 30 & 30 & 30 & 30 & 30 \\
\hline Production Time (ns) & 100 & 70 & 70 & 50 & 50 & 70 \\
\hline seed \# (10ns/seed) & 2 & 6 & 2 & 5 & 3 & 4 \\
\hline Total Time (ns) & 150 & 160 & 120 & 130 & 110 & 140 \\
\hline
\end{tabular}

\begin{tabular}{|c|c|c|c|}
\hline Connexin- $46^{*} / 50^{*}$ Models & Heterotypic & Heteromeric (I) & Heteromeric (II) \\
\hline Total Atoms & 363,884 & 361,412 & 361,412 \\
\hline Solvent & 194,964 & 192,498 & 192,498 \\
\hline Lipids & 133,866 & 133,866 & 133,866 \\
\hline Protein & 34,626 & 34,626 & 34,626 \\
\hline Ions & 428 & 422 & 422 \\
\hline \multirow[t]{2}{*}{ Modelled Residues } & $2-97 ; 154-234$ & $2-97 ; 154-234$ & $2-97 ; 154-234$ \\
\hline & $2-97 ; 142-222$ & $2-97 ; 142-222$ & $2-97 ; 142-222$ \\
\hline Point Group Symmetry & $\mathrm{C} 6$ & C3 & D3 \\
\hline \multicolumn{4}{|l|}{ Simulation Conditions } \\
\hline Simulation Box $(\AA)$ & $152 \times 152 \times 176$ & $152 \times 152 \times 176$ & $152 \times 152 \times 176$ \\
\hline Pressure (atm) & 1 & 1 & 1 \\
\hline Temperature (K) & 310 & 310 & 310 \\
\hline Time Step (fs) & 2 & 2 & 2 \\
\hline Equilibration Time (ns) & 30 & 30 & 30 \\
\hline Production Time (ns) & 80 & 70 & 70 \\
\hline seed \# & 16 & 4 & 4 \\
\hline Total Time (ns) & 270 & 140 & 140 \\
\hline
\end{tabular}

Supplementary Table 1. Summary of molecular dynamics simulation setup and conditions for Cx50, Cx46, Cx26 and Cx46/50 heteromeric/heterotypic models. Asterisk indicates models that included modification by $n$-terminal acetylation. Connexin- 26 models were derived from PDB 2ZW3 $3^{10}$. 
Local Resolution Assessment of Cx46 Atomic Model versus 3.4A CryoEM Map NTH

\begin{tabular}{|c|c|c|c|c|c|c|c|c|c|c|c|c|c|c|}
\hline G2 & D3 & W4 & S5 & F6 & L7 & G8 & R9 & L10 & L11 & E12 & $\mathrm{N} 13$ & A14 & Q15 & E16 \\
\hline $3.0-4.4$ & $3.0-4.6$ & $2.8-3.2$ & $2.9-3.3$ & 3.4-6.7 & 2.9-3.0 & 2.9-3.9 & $3.2-4.2$ & $3.2-3.3$ & 2.8-3.2 & $3.1-5.4$ & $3.4-5.2$ & $3.1 .-3.5$ & $2.8-3.2$ & $3.6-6.4$ \\
\hline & & & & & & & \multicolumn{8}{|c|}{ TM1 } \\
\hline $\mathrm{H} 17$ & S18 & T19 & V20 & 121 & G22 & K23 & V24 & W25 & L26 & T27 & V28 & L29 & F30 & 131 \\
\hline $3.0-3.3$ & 2.9-3.0 & \begin{tabular}{|l}
$2.8-3.2$ \\
\end{tabular} & \begin{tabular}{|l}
$2.8-3.0$ \\
\end{tabular} & $3.1-3.2$ & \begin{tabular}{|l}
$2.8-2.9$ \\
\end{tabular} & \begin{tabular}{|l|}
$2.8-3.1$ \\
\end{tabular} & \begin{tabular}{|l}
$3.0-3.1$ \\
\end{tabular} & $2.8-3.1$ & $2.8-3.0$ & 3.0-3.4 & $2.8-2.9$ & 2.8-2.9 & $2.8-3.3$ & $2.9-3.4$ \\
\hline \multicolumn{15}{|c|}{ TM1 } \\
\hline F32 & R33 & 134 & L35 & V36 & L37 & G38 & A39 & A40 & A41 & E42 & E43 & V44 & W45 & G46 \\
\hline $2.8-3.0$ & $2.7-3.0$ & $2.8-3.0$ & $2.8-3.0$ & $2.8-3.0$ & $2.9-3.0$ & $2.9-3.0$ & $2.8-2.9$ & $2.9-3.2$ & \begin{tabular}{|l|}
$2.8-2.9$ \\
\end{tabular} & $3.0-9.0$ & $2.8-3.7$ & \begin{tabular}{|l|}
$2.8-2.9$ \\
\end{tabular} & $2.6-2.8$ & $2.8-3.0$ \\
\hline \multicolumn{13}{|c|}{ EC1- } & \multicolumn{2}{|c|}{ EC1 Helix } \\
\hline D47 & E48 & Q49 & S50 & D51 & F52 & T53 & C54 & N55 & T56 & Q57 & Q58 & P59 & G60 & C61 \\
\hline $2.9-3.1$ & $2.8-3.0$ & $2.8-3.0$ & $3.0-3.3$ & $2.9-3.2$ & $2.7-3.0$ & 2.9-3.4 & $3.0-3.2$ & $3.0-3.1$ & 3.1-3.4 & $2.9-3.2$ & $3.0-3.5$ & 3.3-3.6 & $3.0-3.4$ & $3.1-3.5$ \\
\hline \multicolumn{12}{|c|}{ EC1 Helix } & \multicolumn{3}{|c|}{ TM2 } \\
\hline E62 & $\mathrm{N} 63$ & V64 & C65 & Y66 & D67 & R68 & A69 & F70 & P71 & 172 & $\mathrm{~S} 73$ & $\mathrm{H} 74$ & V75 & R76 \\
\hline $3.0-6.6$ & $3.0-3.1$ & $3.0-3.1$ & 2.9-3.2 & $2.7-3.0$ & 3.2-3.7 & $3.0-7.2$ & 2.9-3.0 & 2.7-3.2 & 2.8-3.1 & $2.9-3.1$ & $2.9-3.1$ & 3.0-3.1 & $2.8-2.9$ & $2.9-3.0$ \\
\hline \multicolumn{15}{|c|}{ TM2 } \\
\hline F77 & W78 & V79 & L80 & Q81 & 182 & \begin{tabular}{|l|}
183 \\
\end{tabular} & \begin{tabular}{|l|} 
F84 \\
\end{tabular} & V85 & \begin{tabular}{|l|}
$\mathbf{S} 86$ \\
\end{tabular} & \begin{tabular}{|l|} 
T87 \\
\end{tabular} & P88 & \begin{tabular}{|l|} 
T89 \\
\end{tabular} & L90 & 191 \\
\hline $2.8-3.1$ & $2.8-3.0$ & 2.7-2.9 & \begin{tabular}{|l|}
$2.8-2.9$ \\
\end{tabular} & $2.8-3.1$ & $2.8-3.0$ & $2.7-2.8$ & 2.8-3.0 & 2.7-3.0 & \begin{tabular}{|l|}
$2.8-2.9$ \\
\end{tabular} & $3.0-3.2$ & 2.8-3.3 & \begin{tabular}{|l|}
$2.8-3.1$ \\
\end{tabular} & 3.1-3.3 & $3.1-4.1$ \\
\hline \multicolumn{4}{|c|}{ TM2 } & & & ICL & & & & & \multicolumn{4}{|c|}{ TM3 } \\
\hline Y92 & L93 & G94 & H95 & V96 & L97 & & L142 & L143 & R144 & T145 & Y146 & \begin{tabular}{l|} 
V147 \\
\end{tabular} & F148 & N149 \\
\hline $3.0-3.3$ & $3.0-3.1$ & $3.1-4.0$ & $4.5-7.9$ & $3.1-3.4$ & $3.0-3.2$ & & 3.3-3.8 & $3.3-3.6$ & 3.6-6.1 & $3.3-3.7$ & 2.8-3.3 & 3.2-3.8 & $3.2-7.0$ & $2.9-3.0$ \\
\hline \multicolumn{15}{|c|}{ TM3 } \\
\hline 1150 & $\mid 151$ & F152 & K153 & $\mathrm{T} 154$ & L155 & F156 & E157 & V158 & G159 & F160 & $\mid 161$ & A162 & G163 & Q164 \\
\hline $2.9-3.2$ & $3.2-3.3$ & $3.0-5.8$ & 2.7-3.0 & $3.1-3.2$ & 3.2-3.7 & 3.0-3.7 & $3.0-3.2$ & \begin{tabular}{|l|}
$3.2-3.6$ \\
\end{tabular} & 4.1-4.4 & $2.9-3.3$ & 3.3-3.7 & \begin{tabular}{|l|}
$4.0-4.4$ \\
\end{tabular} & $3.4-4.2$ & $2.9-3.2$ \\
\hline \multicolumn{6}{|c|}{ TM3 } & & & & & & & \multicolumn{3}{|c|}{ EC2 } \\
\hline Y165 & \begin{tabular}{|l|} 
F166 \\
\end{tabular} & L167 & \begin{tabular}{|l|} 
Y168 \\
\end{tabular} & G169 & $\begin{array}{l}\text { F170 } \\
\end{array}$ & Q171 & L172 & K173 & $\begin{array}{l}\text { P174 } \\
\end{array}$ & L175 & Y176 & R177 & C178 & $\begin{array}{l}\text { D179 } \\
\end{array}$ \\
\hline $2.9-5.3$ & $3.1-6.5$ & $2.9-3.0$ & 2.7-3.2 & $2.9-3.0$ & \begin{tabular}{|l|}
$2.9-7.2$ \\
\end{tabular} & 2.9-3.7 & 2.8-2.9 & 2.9-3.5 & 2.8-2.9 & $3.0-3.2$ & 2.8-3.2 & \begin{tabular}{|l|}
$2.8-2.9$ \\
\end{tabular} & $2.9-3.1$ & $3.1-4.0$ \\
\hline \multicolumn{15}{|c|}{ EC2 } \\
\hline R180 & W181 & P182 & C183 & P184 & N185 & T186 & V187 & D188 & C189 & F190 & I191 & S192 & R193 & P194 \\
\hline $3.0-3.7$ & $2.9-3.8$ & $3.1-3.9$ & $3.0-3.3$ & $3.0-3.2$ & 2.8-3.4 & $3.2-3.6$ & $3.2-3.3$ & $3.0-3.2$ & $2.9-3.2$ & $2.7-3.1$ & $2.7-3.2$ & \begin{tabular}{|l|}
$3.0-3.2$ \\
\end{tabular} & $2.9-3.1$ & $2.8-3.1$ \\
\hline \multicolumn{15}{|c|}{ TM4 } \\
\hline T195 & E196 & K197 & T198 & I199 & F200 & 1201 & L202 & F203 & M204 & L205 & A206 & V207 & A208 & C209 \\
\hline $2.9-3.2$ & $2.9-3.0$ & $2.8-3.0$ & $3.1-3.4$ & $2.9-3.0$ & 2.7-2.9 & $2.9-3.1$ & 2.8-3.0 & 2.9-3.1 & 2.8-3.0 & $3.0-3.2$ & \begin{tabular}{|l|}
$3.0-3.2$ \\
\end{tabular} & \begin{tabular}{|l|}
$2.7-2.9$ \\
\end{tabular} & $2.9-3.0$ & $2.9-7.2$ \\
\hline \multicolumn{13}{|c|}{ TM4 } & \multirow{3}{*}{\multicolumn{2}{|c|}{ - CTD }} \\
\hline V210 & S211 & L212 & L213 & L214 & \begin{tabular}{l|} 
N215 \\
\end{tabular} & \begin{tabular}{|l|} 
V216 \\
\end{tabular} & L217 & E218 & 1219 & Y220 & H221 & L222 & & \\
\hline $2.9-3.0$ & $2.9-3.0$ & 2.9-3.0 & $3.0-3.5$ & $2.9-3.0$ & \begin{tabular}{|l|}
$2.8-3.0$ \\
\end{tabular} & 2.9-3.1 & $3.0-3.1$ & 2.9-3.1 & 3.2-3.3 & $3.2-6.6$ & 3.3-3.4 & 3.1-4.4 & & \\
\hline
\end{tabular}

Supplementary Table 2. Local resolution assessment of a calculated map based on the Cx46 atomic model versus the 3.4 A CryoEM density map. Values indicated by each residue correspond to the range of resolutions $(\AA)$ reported for amino acid sidechain densities using BlocRes ${ }^{70}$. Secondary structure and domain labels are indicated for the n-terminal helix (NTH), transmembrane helices (TM1-4) and extracellular domains (EC1-2) intracellular loop (ICL) and C-terminal domain (CTD). 


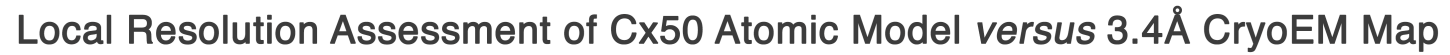

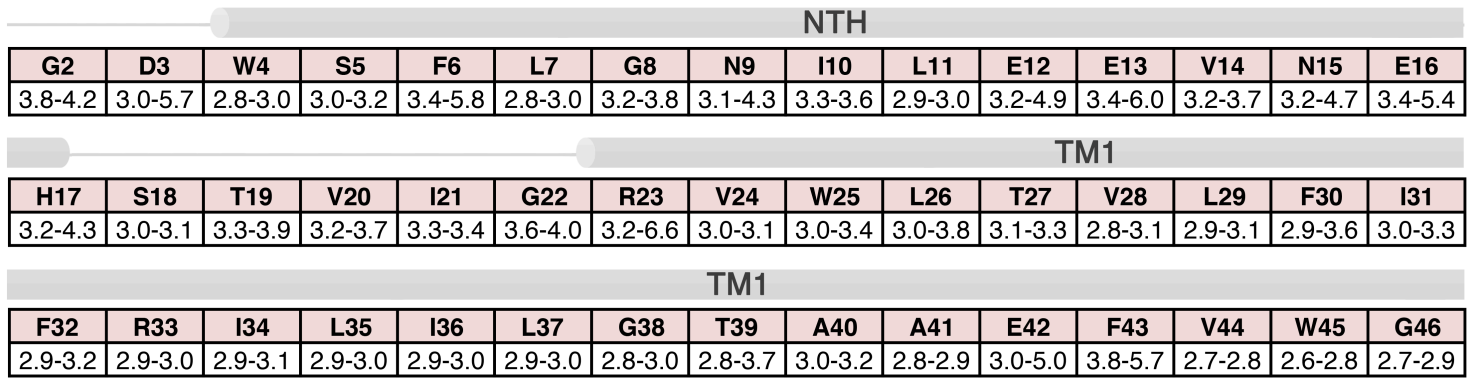

EC1 1

EC1 Helix

\begin{tabular}{|c|c|c|c|c|c|c|c|c|c|c|c|c|c|c|}
\hline D47 & E48 & Q49 & $\mathrm{S} 50$ & D51 & F52 & V53 & C54 & N55 & T56 & Q57 & Q58 & P59 & G60 & C61 \\
\hline $3.0-3.2$ & $2.7-2.9$ & $2.8-3.0$ & $3.0-3.4$ & $3.0-4.3$ & $2.8-3.0$ & $3.0-3.4$ & $3.0-3.2$ & $3.0-3.2$ & \begin{tabular}{|l}
$2.9-3.2$ \\
\end{tabular} & \begin{tabular}{|l}
$2.9-3.1$ \\
\end{tabular} & $3.0-3.4$ & $3.2-3.5$ & $3.0-3.3$ & $3.1-3.4$ \\
\hline \multicolumn{7}{|c|}{ EC1 Helix } & & & & & & \multicolumn{3}{|c|}{ TM2 } \\
\hline E62 & N63 & V64 & C65 & Y66 & D67 & E68 & A69 & F70 & P71 & 172 & S73 & H74 & 175 & R76 \\
\hline $3.1-8.0$ & $3.1-3.2$ & $2.9-3.2$ & $3.8-3.1$ & $2.8-3.0$ & $3.0-3.2$ & $2.7-3.4$ & $2.0-3.5$ & $2.7-3.2$ & $2.8-2.9$ & $2.8-3.1$ & $2.7-3.0$ & $3.0-3.1$ & $2.8-3.0$ & $3.9-3.2$ \\
\hline
\end{tabular}

TM2

\begin{tabular}{|c|c|c|c|c|c|c|c|c|c|c|c|c|c|c|}
\hline L77 & W78 & V79 & L80 & Q81 & 182 & 183 & F84 & V85 & S86 & T87 & P88 & S89 & L90 & V91 \\
\hline
\end{tabular}

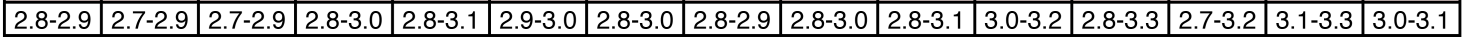

TM2

ICL

TM3

\begin{tabular}{|c|c|c|c|c|c|}
\hline Y92 & L93 & G94 & H95 & A96 & V97 \\
\hline $3.2-3.6$ & $3.0-3.1$ & $3.1-4.1$ & $3.2-8.0$ & $3.3-3.5$ & $3.1-4.3$
\end{tabular}$/$\begin{tabular}{|c|c|c|c|c|c|c|c|}
\hline L154 & L155 & R156 & T157 & Y158 & V159 & C160 & H161 \\
\hline $3.2-3.5$ & $3.2-3.4$ & $3.5-7.0$ & $4.0-4.9$ & $2.9-3.5$ & $3.2-4.0$ & $3.1-4.2$ & $2.8-3.8$ \\
\hline
\end{tabular}

TM3

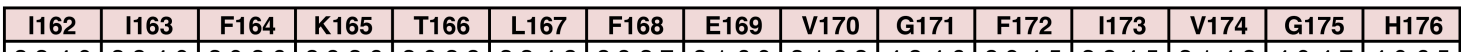

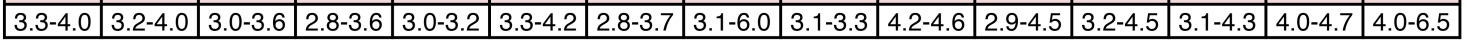

TM3

EC2

\begin{tabular}{|c|c|c|c|c|c|c|c|c|c|c|c|c|c|c|}
\hline Y177 & F178 & L179 & Y180 & G181 & F182 & Q183 & I184 & L185 & P186 & L187 & Y188 & R189 & C190 & S191 \\
\hline
\end{tabular}

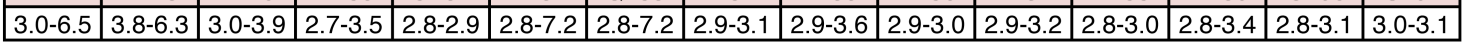

EC2

EC2

\begin{tabular}{|l|l|l|l|l|l|l|l|l|l|l|l|l|l|l|}
\hline R192 & W193 & P194 & C195 & P196 & N197 & V198 & V199 & D200 & C201 & F202 & V203 & S204 & R205 & P206 \\
\hline
\end{tabular}

\begin{tabular}{|c|c|c|c|c|c|c|c|c|c|c|c|c|c|c|}
\hline $3.0-5.7$ & $3.0-4.0$ & $2.9-3.9$ & $3.0-3.2$ & $3.0-3.2$ & $3.2-3.7$ & $3.1-3.7$ & $3.2-3.4$ & $3.0-3.2$ & $3.0-3.1$ & $2.8-2.9$ & $2.8-3.1$ & $3.1-3.2$ & $2.8-2.9$ & $2.9-3.1$ \\
\hline
\end{tabular}

TM4

\begin{tabular}{|c|c|c|c|c|c|c|c|c|c|c|c|c|c|c|}
\hline T207 & E208 & K209 & T210 & I211 & F212 & I213 & L214 & F215 & M216 & L217 & S218 & V219 & A220 & S221 \\
\hline
\end{tabular}

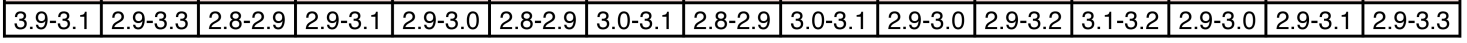

TM4

CTD

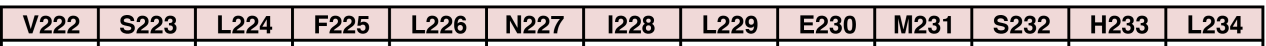

\begin{tabular}{|c|c|c|c|c|c|c|c|c|c|c|c|c|}
\hline $3.0-3.1$ & $2.9-3.0$ & $3.0-3.1$ & $2.7-7.2$ & $2.9-3.0$ & $2.9-3.1$ & $3.0-5.3$ & $3.1-6.0$ & $3.1-3.3$ & $3.1-6.5$ & $3.5-4.4$ & $3.3-3.6$ & $3.3-4.7$ \\
\hline
\end{tabular}

Supplementary Table 3. Local resolution assessment of a calculated map based on the Cx50 atomic model versus the $3.4 \AA$ CryoEM density map. Values indicated by each residue correspond to the range of resolutions $(\AA)$ reported for amino acid sidechain densities using BlocRes ${ }^{70}$. Secondary structure and domain labels are indicated for the n-terminal helix (NTH), transmembrane helices (TM1-4) and extracellular domains (EC1-2) intracellular loop (ICL) and C-terminal domain (CTD). 


\section{References}

1 Goodenough, D. A. \& Paul, D. L. Gap junctions. Cold Spring Harbor perspectives in biology 1, a002576, doi:10.1101/cshperspect.a002576 (2009).

2 Delmar, M. et al. Connexins and Disease. Cold Spring Harbor perspectives in biology, doi:10.1101/cshperspect.a029348 (2017).

3 Garcia, I. E. et al. Connexinopathies: a structural and functional glimpse. BMC Cell Biol 17 Suppl 1, 17, doi:10.1186/s12860-016-0092-x (2016).

4 Aasen, T., Mesnil, M., Naus, C. C., Lampe, P. D. \& Laird, D. W. Gap junctions and cancer: communicating for 50 years. Nat Rev Cancer 16, 775-788, doi:10.1038/nrc.2016.105 (2016).

5 Sosinsky, G. E. \& Nicholson, B. J. Structural organization of gap junction channels. Biochim Biophys Acta 1711, 99-125, doi:10.1016/j.bbamem.2005.04.001 (2005).

6 Sohl, G. \& Willecke, K. Gap junctions and the connexin protein family. Cardiovasc Res 62, 228-232, doi:10.1016/j.cardiores.2003.11.013 (2004).

7 Cottrell, G. T. \& Burt, J. M. Functional consequences of heterogeneous gap junction channel formation and its influence in health and disease. Biochim Biophys Acta 1711, 126-141, doi:10.1016/j.bbamem.2004.11.013 (2005). 
8 Beyer, E. C. \& Berthoud, V. M. Gap junction structure: unraveled, but not fully revealed. F1000Res 6, 568, doi:10.12688/f1000research.10490.1 (2017).

9 Grosely, R. \& Sorgen, P. L. A history of gap junction structure: hexagonal arrays to atomic resolution. Cell Commun Adhes 20, 11-20, doi:10.3109/15419061.2013.775256 (2013).

10 Maeda, S. et al. Structure of the connexin 26 gap junction channel at 3.5 A resolution. Nature 458, 597-602, doi:10.1038/nature07869 (2009).

11 Bennett, B. C. et al. An electrostatic mechanism for $\mathrm{Ca}(2+)$-mediated regulation of gap junction channels. Nat Commun 7, 8770, doi:10.1038/ncomms9770 (2016).

12 Mathias, R. T., White, T. W. \& Gong, X. Lens gap junctions in growth, differentiation, and homeostasis. Physiol Rev 90, 179-206, doi:10.1152/physrev.00034.2009 (2010).

13 Konig, N. \& Zampighi, G. A. Purification of bovine lens cell-to-cell channels composed of connexin44 and connexin50. J Cell Sci 108 ( Pt 9), 3091-3098 (1995).

14 Jiang, J. X. \& Goodenough, D. A. Heteromeric connexons in lens gap junction channels. Proc Natl Acad Sci U S A 93, 1287-1291 (1996).

15 Shearer, D., Ens, W., Standing, K. \& Valdimarsson, G. Posttranslational modifications in lens fiber connexins identified by off-line-HPLC MALDI- 
quadrupole time-of-flight mass spectrometry. Invest Ophthalmol Vis Sci 49, 1553-1562, doi:10.1167/iovs.07-1193 (2008).

16 Wang, Z. \& Schey, K. L. Phosphorylation and truncation sites of bovine lens connexin 46 and connexin 50. Exp Eye Res 89, 898-904, doi:10.1016/j.exer.2009.07.015 (2009).

17 Kwon, T. et al. Molecular dynamics simulations of the Cx26 hemichannel: insights into voltage-dependent loop-gating. Biophys J 102, 1341-1351, doi:10.1016/j.bpj.2012.02.009 (2012).

18 Bai, D. Structural analysis of key gap junction domains--Lessons from genome data and disease-linked mutants. Semin Cell Dev Biol 50, 74-82, doi:10.1016/j.semcdb.2015.11.015 (2016).

19 Koval, M., Molina, S. A. \& Burt, J. M. Mix and match: investigating heteromeric and heterotypic gap junction channels in model systems and native tissues. FEBS Lett 588, 1193-1204, doi:10.1016/j.febslet.2014.02.025 (2014).

20 Unger, V. M., Kumar, N. M., Gilula, N. B. \& Yeager, M. Three-dimensional structure of a recombinant gap junction membrane channel. Science $\mathbf{2 8 3}$, 1176-1180 (1999).

21 Fleishman, S. J., Unger, V. M., Yeager, M. \& Ben-Tal, N. A Calpha model for the transmembrane alpha helices of gap junction intercellular channels. Mol Cell 15, 879-888, doi:10.1016/j.molcel.2004.08.016 (2004). 
22 Gong, X. Q. \& Nicholson, B. J. Size selectivity between gap junction channels composed of different connexins. Cell Commun Adhes 8, 187$192(2001)$.

23 Goldberg, G. S., Valiunas, V. \& Brink, P. R. Selective permeability of gap junction channels. Biochim Biophys Acta 1662, 96-101, doi:10.1016/j.bbamem.2003.11.022 (2004).

24 Varland, S., Osberg, C. \& Arnesen, T. N-terminal modifications of cellular proteins: The enzymes involved, their substrate specificities and biological effects. Proteomics 15, 2385-2401, doi:10.1002/pmic.201400619 (2015).

25 Berneche, S. \& Roux, B. Energetics of ion conduction through the K+ channel. Nature 414, 73-77, doi:10.1038/35102067 (2001).

26 Corry, B. \& Thomas, M. Mechanism of ion permeation and selectivity in a voltage gated sodium channel. J Am Chem Soc 134, 1840-1846, doi:10.1021/ja210020h (2012).

27 Ulmschneider, M. B. et al. Molecular dynamics of ion transport through the open conformation of a bacterial voltage-gated sodium channel. Proc Natl Acad Sci U S A 110, 6364-6369, doi:10.1073/pnas.1214667110 (2013).

28 Srinivas, M. et al. Voltage dependence of macroscopic and unitary currents of gap junction channels formed by mouse connexin50 expressed in rat neuroblastoma cells. J Physiol 517 ( Pt 3), 673-689 (1999). 
29 Hopperstad, M. G., Srinivas, M. \& Spray, D. C. Properties of gap junction channels formed by $\mathrm{Cx} 46$ alone and in combination with $\mathrm{Cx} 50$. Biophys $J$ 79, 1954-1966, doi:10.1016/S0006-3495(00)76444-7 (2000).

30 Oh, S., Rubin, J. B., Bennett, M. V., Verselis, V. K. \& Bargiello, T. A. Molecular determinants of electrical rectification of single channel conductance in gap junctions formed by connexins 26 and 32. J Gen Physiol 114, 339-364 (1999).

31 Trexler, E. B., Bukauskas, F. F., Kronengold, J., Bargiello, T. A. \& Verselis, V. K. The first extracellular loop domain is a major determinant of charge selectivity in connexin46 channels. Biophys J 79, 3036-3051, doi:10.1016/S0006-3495(00)76539-8 (2000).

32 Tong, X. et al. The First Extracellular Domain Plays an Important Role in Unitary Channel Conductance of Cx50 Gap Junction Channels. PLoS One 10, e0143876, doi:10.1371/journal.pone.0143876 (2015).

33 Oh, S., Verselis, V. K. \& Bargiello, T. A. Charges dispersed over the permeation pathway determine the charge selectivity and conductance of a Cx32 chimeric hemichannel. J Physiol 586, 2445-2461, doi:10.1113/jphysiol.2008.150805 (2008).

34 Kronengold, J., Trexler, E. B., Bukauskas, F. F., Bargiello, T. A. \& Verselis, V. K. Pore-lining residues identified by single channel SCAM studies in Cx46 hemichannels. Cell Commun Adhes 10, 193-199 (2003). 
35 Zonta, F., Polles, G., Zanotti, G. \& Mammano, F. Permeation pathway of homomeric connexin 26 and connexin 30 channels investigated by molecular dynamics. J Biomol Struct Dyn 29, 985-998, doi:10.1080/073911012010525027 (2012).

36 Lopez, W. et al. Mechanism of gating by calcium in connexin hemichannels. Proc Natl Acad Sci U S A 113, E7986-E7995, doi:10.1073/pnas.1609378113 (2016).

37 Harris, A. L. \& Contreras, J. E. Motifs in the permeation pathway of connexin channels mediate voltage and $\mathrm{Ca}(2+)$ sensing. Front Physiol $\mathbf{5}$, 113, doi:10.3389/fphys.2014.00113 (2014).

38 Srinivas, M., Calderon, D. P., Kronengold, J. \& Verselis, V. K. Regulation of connexin hemichannels by monovalent cations. J Gen Physiol 127, 6775, doi:10.1085/jgp.200509397 (2006).

39 Trexler, E. B., Bennett, M. V., Bargiello, T. A. \& Verselis, V. K. Voltage gating and permeation in a gap junction hemichannel. Proc Natl Acad Sci U S A 93, 5836-5841 (1996).

40 Suchyna, T. M. et al. Different ionic selectivities for connexins 26 and 32 produce rectifying gap junction channels. Biophys J 77, 2968-2987, doi:10.1016/S0006-3495(99)77129-8 (1999).

41 Veenstra, R. D. Size and selectivity of gap junction channels formed from different connexins. J Bioenerg Biomembr 28, 327-337 (1996). 
42 Veenstra, R. D. et al. Selectivity of connexin-specific gap junctions does not correlate with channel conductance. Circ Res 77, 1156-1165 (1995).

43 Nicholson, B. J. et al. The molecular basis of selective permeability of connexins is complex and includes both size and charge. Braz J Med Biol Res 33, 369-378 (2000).

44 Ebihara, L., Xu, X., Oberti, C., Beyer, E. C. \& Berthoud, V. M. Coexpression of lens fiber connexins modifies hemi-gap-junctional channel behavior. Biophys J 76, 198-206, doi:10.1016/S0006-3495(99)77189-4 (1999).

45 Xin, L. \& Bai, D. Functional roles of the amino terminal domain in determining biophysical properties of Cx50 gap junction channels. Front Physio/ 4, 373, doi:10.3389/fphys.2013.00373 (2013).

46 Luo, Y., Rossi, A. R. \& Harris, A. L. Computational Studies of Molecular Permeation through Connexin26 Channels. Biophys J 110, 584-599, doi:10.1016/j.bpj.2015.11.3528 (2016).

47 Kwon, T., Harris, A. L., Rossi, A. \& Bargiello, T. A. Molecular dynamics simulations of the Cx26 hemichannel: evaluation of structural models with Brownian dynamics. J Gen Physiol 138, 475-493, doi:10.1085/jgp.201110679 (2011).

48 Verselis, V. K., Ginter, C. S. \& Bargiello, T. A. Opposite voltage gating polarities of two closely related connexins. Nature $\mathbf{3 6 8}, 348-351$, doi:10.1038/368348a0 (1994). 
49 Peracchia, C. \& Peracchia, L. L. Inversion of both gating polarity and CO2 sensitivity of voltage gating with D3N mutation of Cx50. Am J Physiol Cell Physiol 288, C1381-1389, doi:10.1152/ajpcell.00348.2004 (2005).

50 Srinivas, M., Kronengold, J., Bukauskas, F. F., Bargiello, T. A. \& Verselis, V. K. Correlative studies of gating in Cx46 and Cx50 hemichannels and gap junction channels. Biophys J 88, 1725-1739, doi:10.1529/biophysj.104.054023 (2005).

51 Xin, L., Nakagawa, S., Tsukihara, T. \& Bai, D. Aspartic acid residue D3 critically determines Cx50 gap junction channel transjunctional voltagedependent gating and unitary conductance. Biophys $J$ 102, 1022-1031, doi:10.1016/j.bpj.2012.02.008 (2012).

52 Beyer, E. C., Ebihara, L. \& Berthoud, V. M. Connexin mutants and cataracts. Front Pharmacol 4, 43, doi:10.3389/fphar.2013.00043 (2013).

53 Pascolini, D. \& Mariotti, S. P. Global estimates of visual impairment: 2010. Br J Ophthalmol 96, 614-618, doi:10.1136/bjophthalmol-2011-300539 (2012).

54 Shiels, A. \& Hejtmancik, J. F. Mutations and mechanisms in congenital and age-related cataracts. Exp Eye Res 156, 95-102, doi:10.1016/j.exer.2016.06.011 (2017).

55 Kistler, J., Christie, D. \& Bullivant, S. Homologies between gap junction proteins in lens, heart and liver. Nature 331, 721-723, doi:10.1038/331721a0 (1988). 
56 Kistler, J., Schaller, J. \& Sigrist, H. MP38 contains the membraneembedded domain of the lens fiber gap junction protein MP70. The Journal of biological chemistry 265, 13357-13361 (1990).

57 White, T. W., Bruzzone, R., Goodenough, D. A. \& Paul, D. L. Mouse Cx50, a functional member of the connexin family of gap junction proteins, is the lens fiber protein MP70. Mol Biol Cell 3, 711-720 (1992).

58 Reichow, S. L. et al. Allosteric mechanism of water-channel gating by Ca2+-calmodulin. Nat Struct Mol Biol 20, 1085-1092, doi:10.1038/nsmb.2630 (2013).

59 Gold, M. G. et al. AKAP2 anchors PKA with aquaporin-0 to support ocular lens transparency. EMBO Mol Med 4, 15-26, doi:10.1002/emmm.201100184 (2012).

60 Reichow, S. L. \& Gonen, T. Noncanonical binding of calmodulin to aquaporin-0: implications for channel regulation. Structure 16, 1389-1398, doi:10.1016/j.str.2008.06.011 (2008).

61 Shevchenko, A., Tomas, H., Havlis, J., Olsen, J. V. \& Mann, M. In-gel digestion for mass spectrometric characterization of proteins and proteomes. Nat Protoc 1, 2856-2860, doi:10.1038/nprot.2006.468 (2006).

62 Yang, B. et al. Identification of cross-linked peptides from complex samples. Nat Methods 9, 904-906, doi:10.1038/nmeth.2099 (2012). 
63 Myers, J. B. et al. The CaMKII holoenzyme structure in activationcompetent conformations. Nat Commun 8, 15742, doi:10.1038/ncomms15742 (2017).

64 Tang, G. et al. EMAN2: an extensible image processing suite for electron microscopy. J Struct Biol 157, 38-46, doi:10.1016/j.jsb.2006.05.009 (2007).

65 Mastronarde, D. N. Automated electron microscope tomography using robust prediction of specimen movements. J Struct Biol 152, 36-51, doi:10.1016/j.jsb.2005.07.007 (2005).

66 Zheng, S. Q. et al. MotionCor2: anisotropic correction of beam-induced motion for improved cryo-electron microscopy. Nat Methods 14, 331-332, doi:10.1038/nmeth.4193 (2017).

67 Zhang, K. Gctf: Real-time CTF determination and correction. J Struct Biol 193, 1-12, doi:10.1016/j.jsb.2015.11.003 (2016).

68 Voss, N. R., Yoshioka, C. K., Radermacher, M., Potter, C. S. \& Carragher, B. DoG Picker and TiltPicker: software tools to facilitate particle selection in single particle electron microscopy. J Struct Biol 166, 205-213 (2009).

69 Scheres, S. H. RELION: implementation of a Bayesian approach to cryoEM structure determination. J Struct Biol 180, 519-530, doi:10.1016/j.jsb.2012.09.006 (2012). 
70 Heymann, J. B. \& Belnap, D. M. Bsoft: image processing and molecular modeling for electron microscopy. J Struct Biol 157, 3-18, doi:10.1016/j.jsb.2006.06.006 (2007).

71 Emsley, P. \& Cowtan, K. Coot: model-building tools for molecular graphics. Acta Crystallogr. D60, 2126-2132 (2004).

72 Adams, P. D. et al. PHENIX: a comprehensive Python-based system for macromolecular structure solution. Acta Crystallogr. D66, 213-221 (2010).

73 Chen, V. B. et al. MolProbity: all-atom structure validation for macromolecular crystallography. Acta Crystallogr D Biol Crystallogr 66, 12-21, doi:10.1107/S0907444909042073 (2010).

74 Pettersen, E. F. et al. UCSF Chimera--a visualization system for exploratory research and analysis. J Comput Chem 25, 1605-1612, doi:10.1002/jcc.20084 (2004).

75 Humphrey, W., Dalke, A. \& Schulten, K. VMD: visual molecular dynamics. J Mol Graph 14, 33-38, 27-38 (1996).

76 Locke, D., Bian, S., Li, H. \& Harris, A. L. Post-translational modifications of connexin26 revealed by mass spectrometry. Biochem $J$ 424, 385-398, doi:10.1042/BJ20091140 (2009).

77 Grubmuller, H., Heymann, B. \& Tavan, P. Ligand binding: molecular mechanics calculation of the streptavidin-biotin rupture force. Science 271, 997-999 (1996). 
78 Phillips, J. C. et al. Scalable molecular dynamics with NAMD. J Comput Chem 26, 1781-1802, doi:10.1002/jcc.20289 (2005).

79 Klauda, J. B. et al. Update of the CHARMM all-atom additive force field for lipids: validation on six lipid types. J Phys Chem B 114, 7830-7843, doi:10.1021/jp101759q (2010).

80 Best, R. B. et al. Optimization of the additive CHARMM all-atom protein force field targeting improved sampling of the backbone phi, psi and sidechain chi(1) and chi(2) dihedral angles. J Chem Theory Comput 8, 32573273, doi:10.1021/ct300400x (2012).

81 Zuckerman, D. M. Statistical Physics of Biomolecules: An Introduction. (CRC Press; 1 edition, 2010).

82 Im, W., Seefeld, S. \& Roux, B. A Grand Canonical Monte Carlo-Brownian dynamics algorithm for simulating ion channels. Biophys $J$ 79, 788-801, doi:10.1016/S0006-3495(00)76336-3 (2000).

83 Krissinel, E. \& Henrick, K. Inference of macromolecular assemblies from crystalline state. J Mol Biol 372, 774-797, doi:10.1016/j.jmb.2007.05.022 (2007). 


\section{Chapter 4}

The CaMKII holoenzyme structure in activation-competent conformations

This chapter has been published

The CaMKII holoenzyme structure in activation-competent conformations. Nat Commun 8,15742 (2017). Janette B. Myers ${ }^{1 \dagger}$, Vincent Zaegel ${ }^{2 \dagger}$, Steven J. Coultrap $^{2}$, Adam P. Miller ${ }^{1}$, K. Ulrich Bayer ${ }^{2, *}$, Steve L. Reichow ${ }^{1,{ }^{*}}$

Affiliations:

${ }^{1}$ Department of Chemistry, Portland State University, Portland OR 97021

${ }^{2}$ Department of Pharmacology, University of Colorado, Aurora CO 80045

${ }^{\dagger}$ Indicates equal contribution

*Correspondence: ulli.bayer@ucdenver.edu (K.U.B.); reichow@pdx.edu (S.L.R.)

This work was a collaboration between the Reichow lab and Dr. Ulrich Bayer's lab (CU Denver). Steven Coultrap provided purified CaMKII. Janette Myers prepared electron microscopy grids, collected data and performed image processing to generate the resulting 3D structures. Adam Miller performed the analysis of individual particles. Vincent Zaegel performed cloning and functional studies. Steve Reichow and Ulrich Bayer directed the project. 


\section{Abstract}

The $\mathrm{Ca}^{2+} /$ calmodulin-dependent protein kinase II (CaMKII) assembles into large 12-meric holoenzymes, which is thought to enable regulatory processes required for synaptic plasticity underlying learning, memory and cognition. Here, we used single particle electron microscopy (EM) to determine a pseudoatomic model of the CaMKIla holoenzyme in an extended and activation-competent conformation. The holoenzyme is organized by a rigid central hub complex, while positioning of the kinase domains is highly flexible, revealing dynamic holoenzymes ranging from 15-35 $\mathrm{nm}$ in diameter. While most kinase domains are ordered independently, $\sim 20 \%$ appear to form dimers and $<3 \%$ are consistent with a compact conformation. An additional level of plasticity is revealed by a small fraction of bona-fide 14-mers $(<4 \%)$ that may enable subunit exchange. Biochemical and cellular FRET studies confirm that the extended state of CaMKIla resolved by EM is the predominant form of the holoenzyme, even under molecular crowding conditions. 


\section{Introduction}

The $\mathrm{Ca}^{2+} /$ calmodulin(CaM)-dependent protein kinase II (CaMKII) $\alpha$ isoform is a central mediator of synaptic plasticity that underlies learning, memory and $\operatorname{cognition}^{1-3}$. A hallmark feature of CaMKII regulation is autophosphorylation at T286, which generates $\mathrm{Ca}^{2+} / \mathrm{CaM}$-independent "autonomous" kinase activity that persists even when the initial $\mathrm{Ca}^{2+}$-stimulus subsides. T286-phosphorylation has been described as a form of molecular memory, and is indeed required for normal learning and memory, as well as for two opposing forms of synaptic plasticity, long-term potentiation (LTP) and depression (LTD $)^{4,5}$. Another form of molecular memory by CaMKII is formed through the regulated binding to the NMDA-type glutamate receptor subunit GluN2B, which can also generate autonomous activity ${ }^{6}$. This CaMKII/GluN2B binding is specifically required for maintenance of synaptic strength ${ }^{7}$ and for normal LTP but not LTD ${ }^{8}$.

Both forms of molecular memory are enabled by the CaMKII holoenzyme structure, which is assembled by the association of 12 subunits via their Cterminal association domains (also termed hub domains). GluN2B binding is mediated by the kinase domain, but is dramatically more efficient for holoenzymes compared to monomers ${ }^{6,9,10}$. T286-autophosphorylation occurs as an inter-subunit reaction within the holoenzyme, by a mechanism that enables frequency-detection by CaMKII ${ }^{11-13}$. Additionally, holoenzyme formation is thought to enable structural functions of CaMKII ${ }^{14-16}$. 
Several crystal structures of individual CaMKII domains have been described ${ }^{17-}$ 22. However, the structure of the holoenzyme assembly that determines the relative positioning of the kinase domains to each other remains elusive. The principle domain sequence of a CaMKII subunit is illustrated in (Fig. 1a), with an $\mathrm{N}$-terminal kinase domain, followed by a short regulatory domain that contains T286, the variable linker domain that is subject to alternative splicing, and the Cterminal hub domain. A recent crystallographic model has been described for an artificial CaMKIla construct in which the variable linker domain was completely deleted (linker-less) ${ }^{23}$. After bacterial expression, this linker-less construct formed a compact conformation, in which the kinase/regulatory domains are packed closely against the central hub complex. However, in this compact conformation, the regulatory domains are inaccessible to $\mathrm{Ca}^{2+} / \mathrm{CaM}$-stimulation. Thus, in order to make the holoenzyme competent for activation by $\mathrm{Ca}^{2+} / \mathrm{CaM}$, it has been proposed that at least one alternative extended conformation must exist, even under basal-state conditions ${ }^{1,18,23}$. Additionally, in order to enable the intersubunit autophosphorylation at T286, there must be a conformation with a flexible kinase domain positioning that allows for one kinase domain to access the regulatory domain of its neighbor. Such flexibility could either be induced by $\mathrm{Ca}^{2+} / \mathrm{CaM}$-stimulation or be an intrinsic property of the activation-competent conformation of a holoenzyme that is present even in its basal state.

For the compact CaMKII conformation, the biological occurrence and implications remains to be elucidated, especially in context of a naturally occurring full-length 
CaMKII that contains the linker region. However, it has been suggested that an equilibrium between the compact conformation and an additional activationcompetent conformation would shape the CaMKII activation characteristics: it would make the stimulation by $\mathrm{Ca}^{2+} / \mathrm{CaM}$ cooperative and thereby also fine-tune the frequency-dependent response of CaMKII to $\mathrm{Ca}^{2+}$-oscillation ${ }^{23}$. However, an alternative mechanism for the cooperativity of $\mathrm{Ca}^{2+} / \mathrm{CaM}-$ stimulation has also been suggested. This alternative model is based on a previous crystal structure of isolated kinase/regulatory domains in which two kinase subunits dimerized via a coiled-coil interaction formed by their $\mathrm{Ca}^{2+} / \mathrm{CaM}$-binding regulatory domains ${ }^{19}$. Thus, it is proposed that dimerization would prevent direct activation of this form as well. However, binding of CaM to one of these paired subunits would facilitate subsequent cooperative CaM-binding to the second subunit that was part of the dimer pair ${ }^{19}$. While the compact holoenzyme conformation and the kinase domain dimers cannot occur at the same time, both could be part of an equilibrium exchange. However, any equilibrium must include at least one additional activation-competent conformation in which the $\mathrm{Ca}^{2+} / \mathrm{CaM}$ binding sites are accessible for holoenzyme activation.

Here, we set out to investigate the CaMKIla holoenzyme structure and potential conformational equilibrium using a combination of single particle electron microscopy (EM) and functional mutation studies. Briefly, we describe a 3D EM structure of an activation-competent CaMKIla holoenzyme in an extended-state conformation at pseudo-atomic resolution. Single particle analysis revealed a 
high degree of flexibility for kinase domain positioning, with almost none of the individual particles completely matching the average structure. Within this continuum of conformational states, a compact conformation was found for less than $3 \%$ of the individual kinase domains, but various dimer-pair arrangements were suggested for as much as $\sim 20 \%$ of the kinase domains. Biochemical and cellular studies indicate that the extended state structures described by EM predominate even under conditions of molecular crowding, and point to an important role of the intrinsically disordered linker domain in facilitating cooperative activation for wild type holoenzymes.

\section{Results}

\section{An extended activatable state of CaMKIla revealed by EM}

CaMKII holoenzymes were prepared for electron microscopy (EM) using negative stain (Fig. 1). This procedure enabled high-contrast single particle image analysis, which turned out to be important for assessing the extreme structural heterogeneity in holoenzyme architecture determined here. In raw micrographs, individual particles appear as "flower-like" shapes with a defined central ring of protein density surrounded by an array of smaller densities, "petals" (Fig. 1c,d). The central ring of density appears as a six-pointed star (diameter $=\sim 110 \AA)$ with a distinct central pore $($ diameter $=\sim 25 \AA)($ Fig. 1d, blue outline). Surrounding the ring structure, up to twelve punctate densities are observed at an approximate diameter of $\sim 24-28 \mathrm{~nm}$ (Fig. 1d, yellow circles). These peripheral features correspond to the twelve kinase domains, assembled 

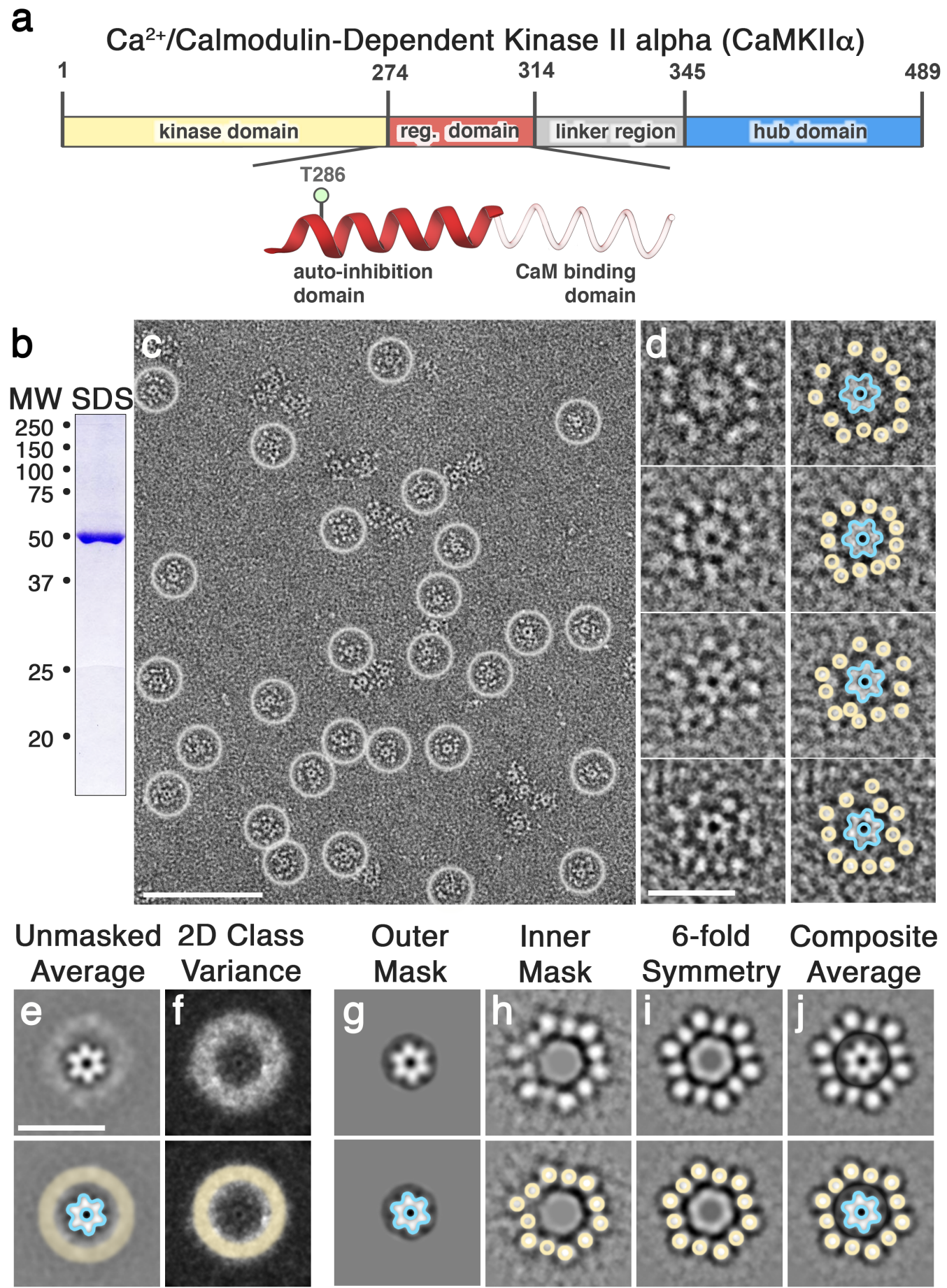

Figure 1. An extended form of the CaMKlla holoenzyme resolved by single particle EM. (a) Diagram of the CaMKIla domain architecture (numbering of human isoform). Inset, shows a secondary structure diagram of the CaMKIla regulatory domain. The coil region (faded red) represents a region that is disordered in the absence of calmodulin ${ }^{21}$. The site of autophosphorylation (T286) involved in regulating autonomous activity is indicated. (cont'd page 124) 
by the dodecameric hub complex ${ }^{24}$. The central hub was found to adopt a single highly preferred orientation; by contrast, the configuration of the radial kinase domain densities appeared highly variable with respect to the central hub feature (Fig. 1c,d). These general features were consistently observed with a variety of negative stain reagents and in vitrified specimens (Supplementary Fig. 1, 2).

Two-dimensional classification and projection averaging produced highly populated classes with well-defined hub assemblies and clear six-fold symmetry. However, the peripheral kinase densities were not resolved in these most populated classes. Rather, these domains appeared as a halo of density surrounding the central hub (Fig. 1e). The halo feature corresponds to a region of high variance in these 2D class averages (Fig. 1f). Inspection of the individual

Figure 1, cont'd. (b) SDS-PAGE of purified human CaMKIla stained with Coomassie blue migrating at the expected molecular weight (MW $\sim 50 \mathrm{kDa}$ ). (c) Electron micrograph of negatively stained CaMKIla particles. Contrast of protein is white on a dark background. Individual particles are indicated by white circles. Scale bar $=100 \mathrm{~nm}$. (d) Enlarged view of individual particles with the hub complex (blue outline) and twelve kinase domains (yellow circle) indicated. Scale bar $=25 \mathrm{~nm}$. (e, f) Single particle averaging and image analysis. Scale bar $=25 \mathrm{~nm}$. (e) Representative $2 D$ projection average of unmasked particles. The central hub complex is clearly defined (blue outline), while the radial kinase domains appear as a diffuse halo (yellow circle). (f) $2 D$ variance map of data in panel e. A region of high variance (white pixels) corresponds with the blurred region observed in $2 D$ class averages (yellow circle). (g) Representative $2 D$ projection average using an applied image mask (75 $A$ outer radius). The 6-fold symmetric hub complex is clearly defined (blue outline). (h) Representative $2 D$ projection average with an applied mask to remove contribution of the hub domain during the alignment procedure (50 $\AA$ inner radius mask). Twelve kinase domains are visualized at an approximate radius of $24-28 \mathrm{~nm}$ (yellow circles). (i) Class average in panel $\boldsymbol{h}$ with applied 6-fold rotational averaging indicates a pseudo-symmetric kinase domain organization. (j) Composite image of panels $\boldsymbol{g}$ and $\boldsymbol{i}$ with the identified hub complex (blue outline) and kinase domains (yellow outline) indicated. 
particles within these classes confirmed that this diffuse signal is produced by the variable arrangement of kinase domains. Separating this $\sim 10,000$ particle image dataset into a larger number of 2D class averages (e.g. $>200-500$ classes) was only partially successful at resolving holoenzyme structures. The resulting distribution of class averages displayed either resolved hub domains and unresolved kinase domains, or partially resolved kinase domains and unresolved hub domains (Fig. 1e and Supplementary Fig. 1, 2). These results were considered indicative of a central hub domain complex that is connected to twelve peripheral kinase domains by disorganized or flexible polypeptide chains, thus preventing their co-alignment. Therefore, alternative 2D classification approaches were explored to deconvolute the structural arrangement(s) of the holoenzyme, by focusing on each of these domains separately. The results are described below.

Focused 2D classification of the hub domain was performed using a circular image mask (outer radius $=75 \AA$ ). The vast majority of these masked particles classified as six-fold symmetric structures, with dimensions matching the crystallized dodecameric hub domain assembly ${ }^{21-23}$ (Fig. 1g and Supplementary Fig. 3). In the next round of focused 2D classification, a new image mask was applied to remove the density representing the central hub complex (50 $\AA$ inner radius). This approach now clearly resolved up to twelve individual kinase domain densities, arranged radially along a diameter of $\sim 24-28 \mathrm{~nm}$ (Fig. $1 \mathrm{~h}$ and Supplementary Fig. 4). In a few classes, an apparent pseudo-symmetric 
arrangement of kinase densities was observed (as in Fig. 1h), and supported by rotational averaging (Fig. 1i). A composite of Fig. 1g,h provides an enhanced view of CaMKIla holoenzymes observed in raw micrographs, represented by a dodecameric hub complex surrounded by an array of twelve independently arranged kinase domains (Fig. 1j). In addition to this representative average structure, a variety of other kinase domain arrangements (including asymmetric forms) where also observed in single particle images and in 2D class averages (Fig. 1d and Supplementary Fig. 4). Here and in the following section, we have focused on structures where all twelve kinase domains are clearly resolved. In later sections, we describe additional alternative conformational forms that were identified for the basal-state holoenzyme in more detail.

\section{Pseudo-atomic model of CaMKIla in a fully activatable state}

A three-dimensional (3D) EM density map was obtained at $20 \AA$ resolution by using a combination of tilted-series tomographic imaging and 3D masking routines (Fig. 2a and Supplementary Movie 1). A pseudo-atomic model of the CaMKIla holoenzyme was constructed by fitting Protein Data Bank (PDB) coordinates for the dodecameric hub complex (PDBID $5 I G 3^{22}$, blue ribbon) and isolated kinase/regulatory domain (PDBID 2VZ6 ${ }^{21}$, yellow and red ribbon) into the EM density map (see Methods and Supplementary Fig. 5). The central density of the EM map is well defined by the crystal structure of the dodecameric hub complex, formed by residues $345-472$ (cross-correlation $=0.95$ at 20 Å resolution). The structured $\mathrm{N}$-terminal kinase/regulatory domain, containing 

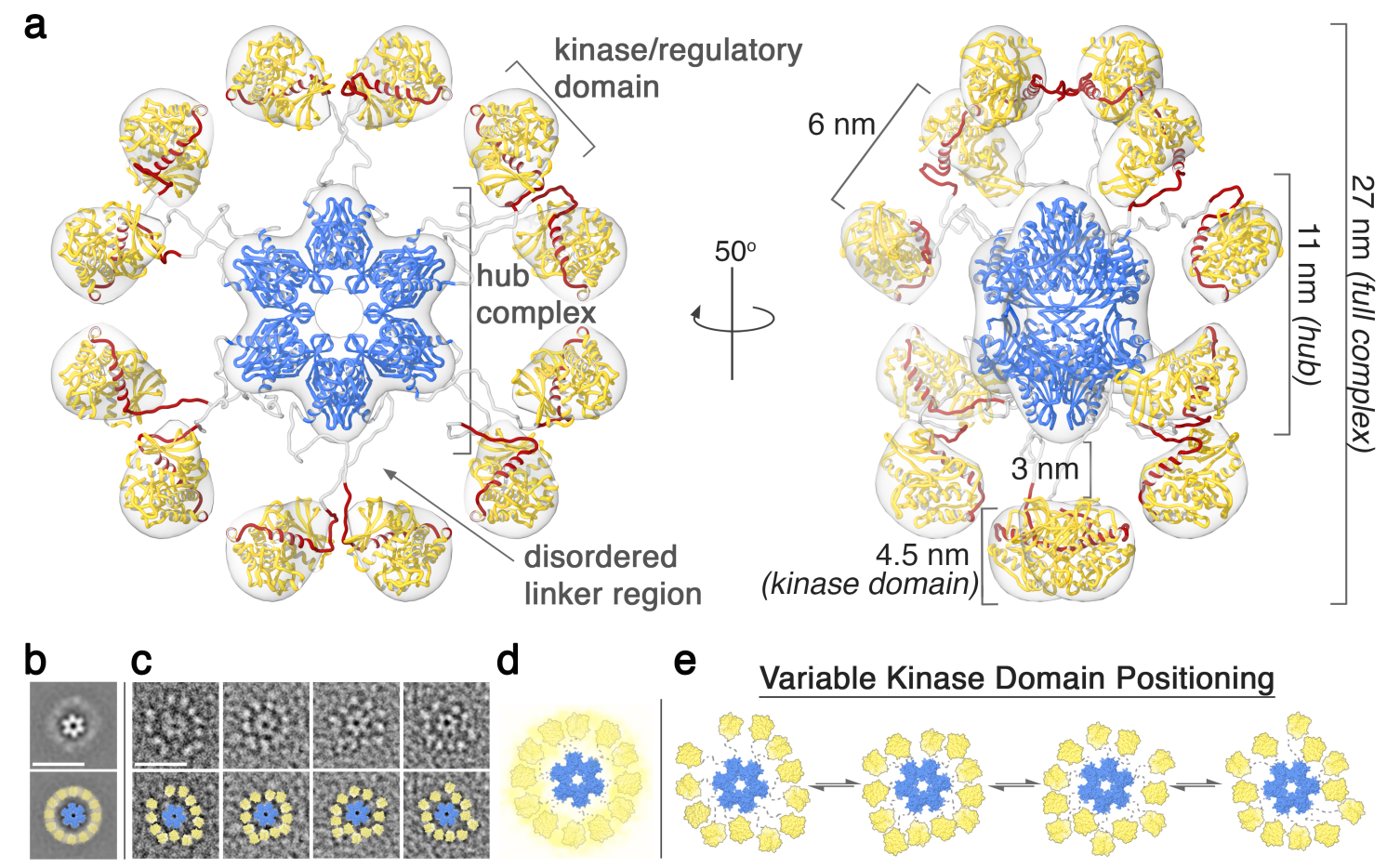

Figure 2: 3D-reconstruction and pseudo-atomic model of the CaMKIla holoenzyme. (a) 3D-reconstruction of the CaMKlla holoenzyme (gray transparent) refined to $\sim 20 \AA$ resolution. A pseudo-atomic model of the dodecameric holoenzyme (colored as in Fig. 1a) was constructed using previously determined crystal structures corresponding to the dodecameric CaMKIla hub complex (blue ribbon, PDBID 5IG322; residues 345 - 472) and isolated kinase/regulatory domain (yellow/red ribbon, PDBID 2VZ6 ${ }^{21}$; residues 13 - 300). Residues 301 - 344 were modeled as disordered linkers connecting each kinase domain to the nearest hub domain. Residues $274-$ 314 correspond to the regulatory domain (red), containing a proximal autoinhibitory segment and distal calmodulin binding site. (b, c) Unmasked $2 D$ class average and single particle images (as shown in Fig. 1) with crystal structures of the hub complex (blue surface) and kinase/regulatory domain (yellow surface) fit into the EM densities. A yellow halo in panel $\boldsymbol{b}$ represents the diffuse positioning of kinase domains in the $2 D$ class average. Scale bars $=25 \mathrm{~nm}$. (d, e) Enlarged view of the fit domains in panels $\boldsymbol{b}, \boldsymbol{c}$ illustrating the proposed structural equilibrium and variable kinase domain arrangements observed in single particle images. Flexible linkers connecting individual kinase domains to the central hub complex are represented as grey dotted lines. 
residues $13-300$, was best fit computationally into each of the radial densities with the regulatory domain (red ribbon in Fig. 2a) oriented toward the central hub complex (cross-correlation $=0.98$ at $20 \AA$ resolution). Neighboring kinase domains were fit separately to reflect the dihedral symmetry imposed by the hub complex and 3D refinement.

The model of the holoenzyme was completed by connecting the structured kinase/regulatory domains to the central hub complex by flexible linkers ${ }^{25}$ (Supplementary Fig. 5). The modeled linkers are not resolved by the EM map, and were therefore inferred by the following substantiating evidence. The crystallized construct of the human CaMKIla kinase/regulatory domain used for our model included the entire regulatory domain (residues $274-314$, Fig. 1a). However, residues $301-314$ (containing the distal region of the CaM binding site) were found to be disordered in the crystal structure ${ }^{21}$. The distal region of the CaM binding site has been shown to be unstructured by solution state EPR studies $^{26}$ and crystallographic analysis of the human $\beta, y$ and $\delta$ isoforms as well $^{21}$. Primary sequence analysis further indicates residues $315-344$ comprising the CaMKIla variable linker region are intrinsically disordered ${ }^{27}$. Therefore, for the basal-state holoenzyme structure determined here, each $\mathrm{N}$ terminal kinase/regulatory domain was covalently connected to the nearest Cterminal hub domain by a random coil peptide chain, containing residues 301 344 (Fig. 2a, red and grey coil). 
The pseudo-atomic model presented here provides a representative average view of the CaMKIla holoenzyme in an extended (and activatable) conformation. In this form of the holoenzyme, the kinase domains are arranged independently from each other and removed from the central hub complex. The model implies that each kinase domain is tethered to the central hub complex by a flexible chain of amino acids (residues 301 - 344). Each kinase domain is separated from the central hub complex, extending the radius of the model to $\sim 135 \AA$. Neighboring kinase domains are separated from each other by $\sim 60 \AA$ (center-tocenter distance). The co-planar arrangement of kinase domains is consistent with previous SAXS analysis ${ }^{19,23}$. However, in the EM model, each of the kinase/regulatory domains are displaced from the hub complex by $\sim 30 \AA$ (edgeto-edge) separation (Fig. 2a). In this extended form of the holoenzyme, the CaM binding sites ${ }^{21}$ (residues $294-314$, red ribbon/coil) are solvent exposed and thus positioned for activation by the $\mathrm{Ca}^{2+} / \mathrm{CaM}$-stimulus. Therefore, the described structure represents an activation-competent state of the CaMKII holoenzyme.

\section{CaMKIla forms a continuum of activation-competent structures}

Based on single particle image analysis and 2D classification it is apparent that the flexible linker regions facilitate the formation of a variety of other conformational states that do not completely match the averaged 3D reconstruction. This conformational variability is appreciated when crystal structures of individual domains are fitted into the densities of individual particles images (Fig. 2c,e). To quantitate the variable CaMKIla domain architecture, we 
conducted a series of measurements and statistical analysis on individual particle images obtained from raw micrographs. This methodology was facilitated by the high contrast provided by the negative staining technique and preferred orientation of particles on the EM grid.

In the first set of measurements, the radial extension for each kinase domain (i.e., kinase radius) was defined from the center of the hub complex to the outer radius of each kinase domain (Fig. 3a, inset). A histogram obtained from 927 measurements shows a Gaussian distribution of kinase domain radii, with a mean value of $\sim 127 \AA( \pm 16 \AA$ standard deviation) (Fig. 3a). This data is consistent with the particle radius obtained by 3D reconstruction $(\sim 135 \AA)$. As the flexible linker region is not directly visible by EM, the extension of the linker itself was estimated by accounting for the volume occupied by the ordered hub complex ( $\sim 55 \AA$ radius) and kinase/regulatory domains ( $\sim 45 \AA$ diameter). These calculations show that the linker connecting these structured domains adopts an average extension of $\sim 27 \AA$, which compares well to the $\sim 30 \AA$ (edge-to-edge) separation of hub and kinase domain densities in our averaged 3D reconstruction. Theoretical considerations based on random walk theory predict an average extension provided by a 45 residue random coil chain to be $\sim 24 \AA$ (3.5 $\AA \times \sqrt{ } \mathrm{N}_{\text {residues }}{ }^{28}$ ). The Gaussian distribution of measured radii, and correspondence to the expected theoretical value of a random chain model further support the notion that each kinase domain is freely tethered to the hub 

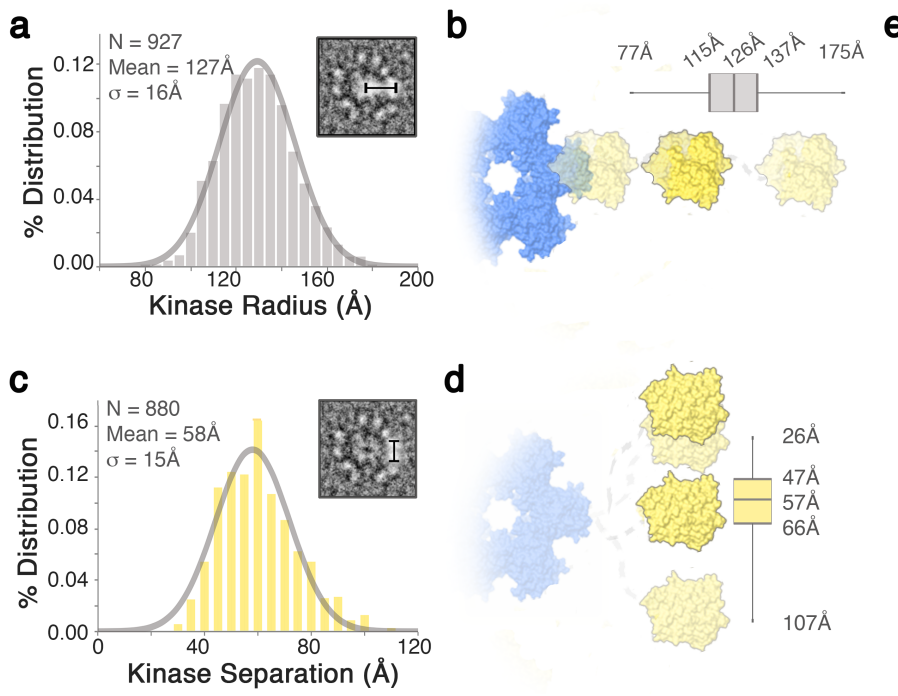

d
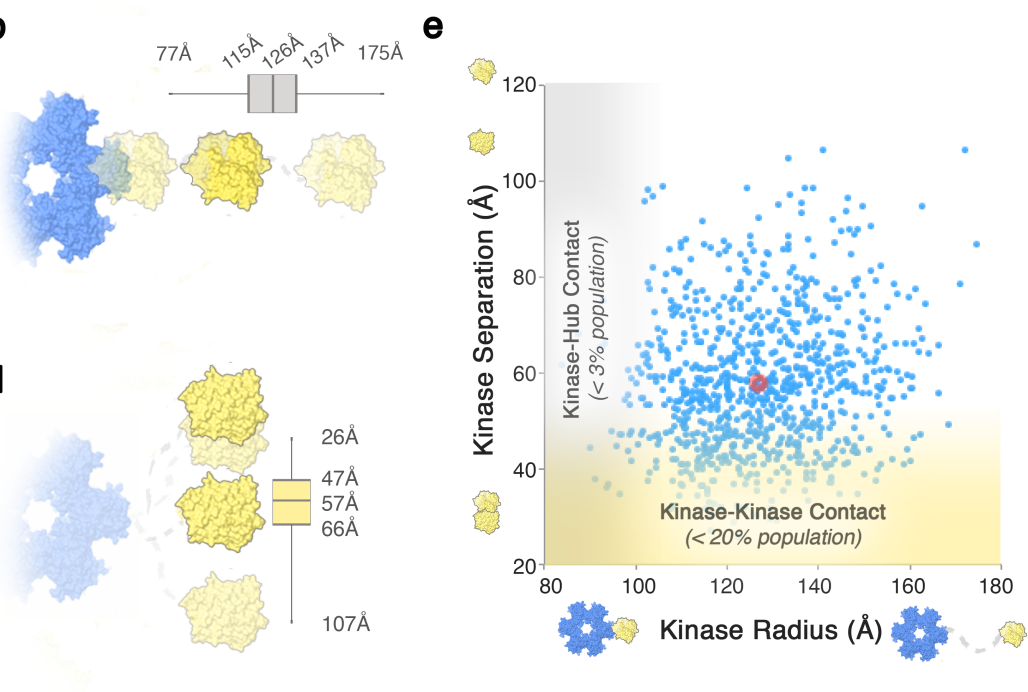

Figure 3: Structural diversity of CaMKIla kinase domain arrangements measured from single particle images. (a) Histogram of measured radius for individual kinase domains. A Gaussian curve fit to the histogram distribution is shown (grey line). (b) Illustration and whisker plot representation of the distribution of kinase radius measurements in panel a. Kinase domains (yellow) are illustrated to represent the minimum, maximum and average distance from the hub domain complex (blue). (c) Histogram of measured distance separating neighboring kinase domains (center-to-center). A Gaussian curve fit to the histogram distribution is shown (grey line). (d) Illustration and whisker plot representation of the distribution of kinase separation measurements in panel c. Kinase domains are illustrated to represent the minimum, maximum and average kinase separation distances. Inset, in a, c illustrate the distance measurement made using raw particle images. Whiskers in $\boldsymbol{b}$, d indicate the minimum and maximum values, and boxes indicate the $25 \%, 50 \%$ and $75 \%$ values of the distribution. (e) Correlation map of kinase radius versus kinase separation distance. This analysis revealed no statistical correlation between these values (correlation coefficient $=0.2$ ). The average of the two parameters is shown in red. Grey and yellow shading indicates regions of the correlation map where kinase domain positioning is consistent with steric contact with the hub domain and/or kinase-kinase contact, respectively. 
complex by an intrinsically flexible linker. In this context, each CaMKII subunit is able to independently sample a continuum of conformations that provide kinase domain extensions ranging from compact forms (minimum radius $=77 \AA$ ) to highly extended forms (maximum radius $=175 \AA$ ) $($ Fig. 3b).

\section{A fully compact CaMKIla architecture is not observed by EM}

A prominent model of a linker-less CaMKII construct has been determined by $\mathrm{X}$ ray crystallography, revealing a highly compact structure where all twelve kinase domains are packed tightly against the central hub complex ${ }^{23}$. As this compact structure is proposed to have a role in the cooperative activation mechanism of CaMKII, we attempted to recover this form of the holoenzyme using our focused 2D classification methods (using an outer mask radius $=110 \AA$ ). However, this approach did not identify any structures consistent with 2D back-projections of the linker-less crystal form (Supplementary Fig. 3). This result was corroborated by our statistical analysis performed on individual particle images. A particle radius less than $\sim 100 \AA$ would potentially place kinase domains in steric contact with the central hub complex. Statistical analysis identified only a small fraction of individual subunits with kinase domain radii that fall within this category $(<3 \%$ of kinase domains with radius $<100 \AA$ ) (Fig. 3e). Together, these data indicate that an interaction between the hub and kinase domain may only occur for a minor fraction of individual subunits in full-length CaMKIla holoenzymes, at least under the conditions required for EM. 


\section{Evidence for meta-stable CaMKII kinase domain dimerization}

A second model of cooperative activation of CaMKII has been proposed based on the observed dimeric-pairing in the crystal structure of isolated CaMKII kinase domains obtained from C. elegans ${ }^{19}$. We assessed the formation of potential kinase domain dimers by measuring the center-to-center distance separating neighboring kinase domains within our individual particle dataset (Fig. 3c, inset). This analysis yielded a Gaussian distribution of kinase domain separation distances, centered at $\sim 58 \AA$ ( $\pm 15 \AA$ standard deviation) (Fig. 3c). This value is remarkably similar to average separation distances determined by previous FRET studies ${ }^{29}$. Here, we additionally considered the approximate volume occupied by each kinase/regulatory domain ( $45 \AA$ diameter) to indicate that the majority of kinase domains are arranged independently (non-interacting) from their neighboring subunits, as represented by the activation-competent structure determined by EM (Fig. 2a).

In addition to these independent states, a significant population of neighboring kinase domains ( $20 \%$ of the population) were found separated by less than 45 $\AA$ (i.e. within steric contact distance) (Fig. 3d,e). This close proximity of neighboring kinase domains is not simply due to positioning by shorter linker extensions. A distribution plot of kinase domain radius versus kinase domain separation revealed no significant correlation between these values (correlation coefficient $=0.2)($ Fig. 3e). For example, a kinase domain with an extended radius (e.g. $>125 \AA$ ) may be found closely positioned $<45 \AA$ to a neighboring 
domain. Vice versa, a kinase domain with a shorter radius (e.g. $<125 \AA)$ may be separated by $>100 \AA$ from one of its neighboring kinase domains. Together these data suggest neighboring kinase domains may exist in two population states, as isolated independent domains and as meta-stable dimeric pairs.

To assess the various patterns of kinase domain pairing, we categorized the holoenzymes from our same individual image dataset according to the number of putative kinase domain pairs, defined as having neighboring kinase domains separated by $<45 \AA$ (Fig. 4a). This analysis identified a variety of arrangements, consisting of $0-6$ sets of kinase domain pairs per particle. Approximately $10 \%$ of the particles were classified as having all kinase domains unpaired and independently arranged by the holoenzyme (as shown in Fig. 1, 2). Holoenzyme structures with $1-3$ kinase domain pairs appeared to be most common (representing a combined $\sim 60 \%$ of the population), while configurations with all twelve kinase domains arranged as dimers appeared to be rare (represented only $\sim 2.5 \%$ of the population).

These individual particle statistics are in agreement with the results obtained by reference-free 2D classification procedures, where a majority of particles classified into structures where only $9-11$ peripheral kinase densities are resolved (Supplementary Fig. 1 and Supplementary Fig. 4). Although rare, we were able to capture a structure from $2 \mathrm{D}$ classification results that is consistent with an arrangement of the holoenzyme where all twelve kinase domains are 


\section{a Kinase Pairing Arrangments within the CaMKII Holoenzme}
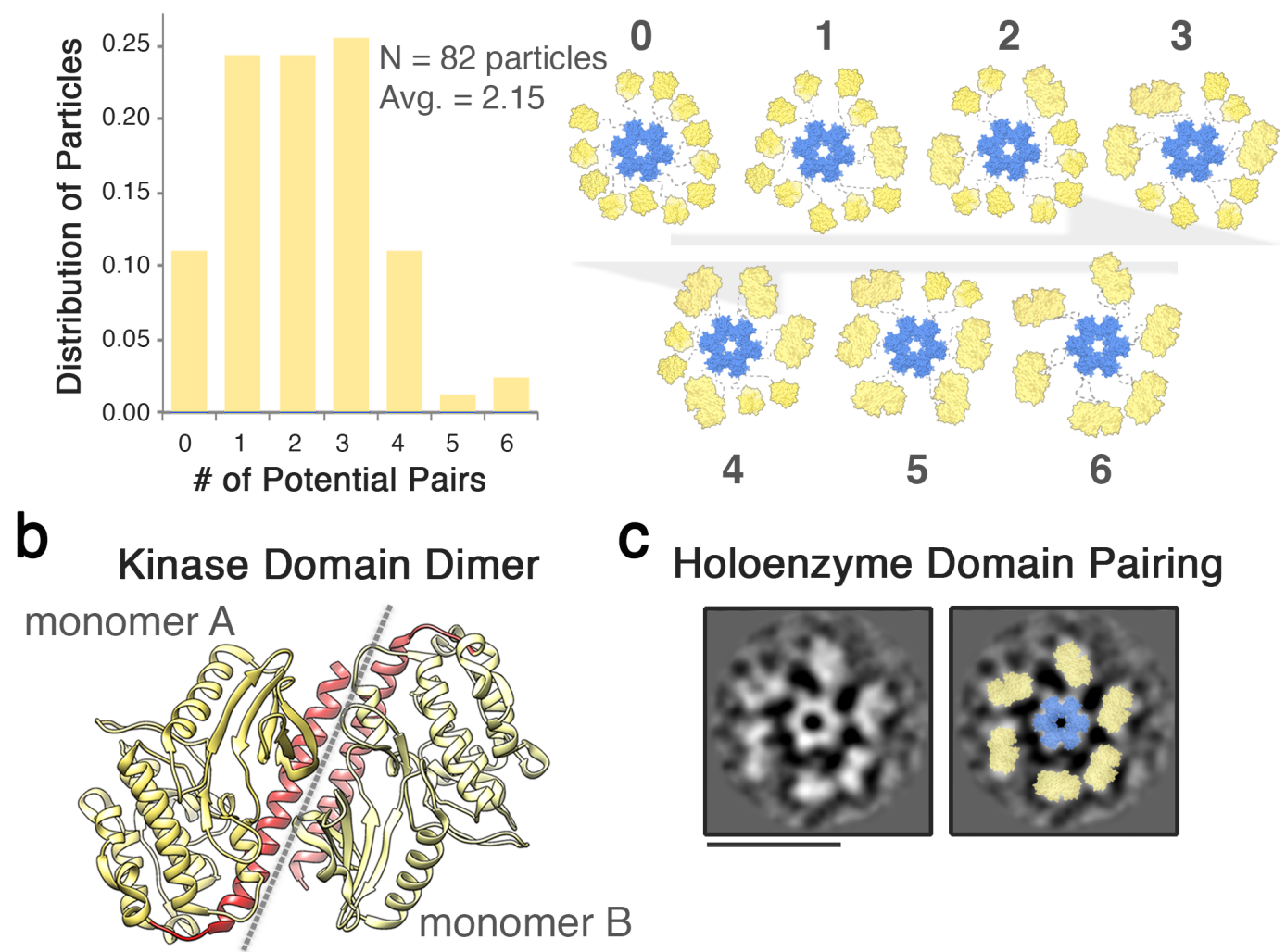

C Holoenzyme Domain Pairing
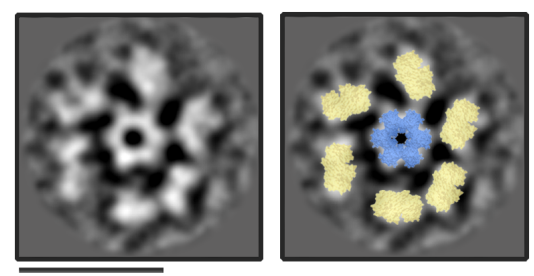

Figure 4: Putative kinase domain pairing in the CaMKIla holoenzyme observed by single particle EM. (a) Left, Distribution of kinase domain pairing arrangements, based on measured inter-domain distances (as described in main text). Holoenzyme structures were categorized as being arranged with $0-6$ kinase domain pairs. Right, Illustration representing holoenzyme structures with various kinase domain pairing arrangements. These structures (and other various arrangements of kinase pairing) are suggested to be in equilibrium with various unpaired kinase arrangements. (b) Crystallographic structure of the C. elegans CaMKlla kinase/regulatory domain (yellow/red ribbon; PDBID 2BDW ${ }^{19}$ ) previously shown to form a dimeric interface involving the regulatory domain (red). (c) Left, Reference-free $2 D$ class average observed for a small population of CaMKIla particles apparently organized with all kinase domains forming paired interactions. Right, Crystal structures of the hub complex (blue surface) and dimeric kinase domains (yellow surface) are fit into the EM densities. Scale bar $=25 \mathrm{~nm}$. 
arranged as kinase dimer pairs (Fig. $\mathbf{4 b , c}$ ). The crystal structure of $C$. elegans CaMKII kinase domain dimer fits well into the peripheral EM densities of this particular 2D class average (Fig. 4c, right). The low population of particles fitting to this individual class ( $<1 \%$ of classified particles) is consistent with the determined population distribution in Fig. $\mathbf{4 a}$.

\section{Wild type CaMKIla remains extended under molecular crowding}

The compact conformation CaMKII holoenzyme model is based on the crystal structure of a linker-less (LL) CaMKIla mutant expressed in bacteria ${ }^{23}$ (Fig. 5a). This compact form of CaMKII is expected to be a non-activatable state because the CaM binding site is buried within the compact kinase/hub interface. However, it was suggested that this compact form enables cooperativity, because CaM binding at one subunit would disrupt the compact conformation at adjacent subunits, thereby facilitating subsequent CaM binding to the neighboring kinase

domains $^{23}$. In support of this notion, the (LL) mutant was reported to have a dramatically increased $\mathrm{EC}_{50}$ for activation by $\mathrm{Ca}^{2+} / \mathrm{CaM}(\sim 500$ fold $)$ and a mildly increased Hill coefficient ( 1.5 fold) compared to full-length wild type CaMKIla, consistent with a compact conformation that decreases $\mathrm{Ca}^{2+} / \mathrm{CaM}$ binding and enhances cooperativity in solution ${ }^{23}$. By contrast, our EM studies conducted on diluted full-length CaMKIla wild type was observed to form a predominant extended conformation, consistent with the less than $3 \%$ of subunits in a potentially compact conformation. However, it was suggested in the previous 

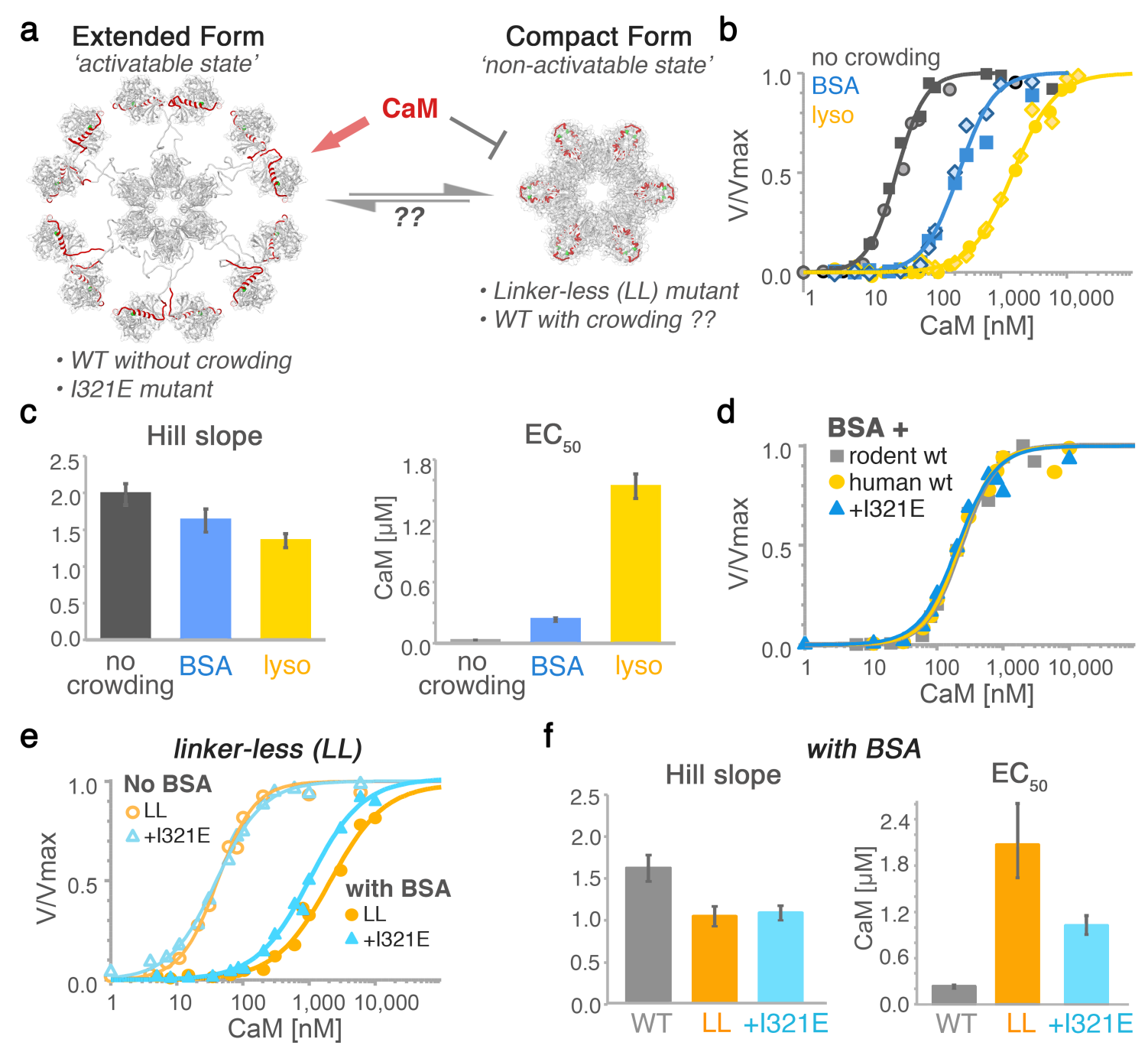
study that molecular crowding conditions, as found within cells, was required to induce a compact conformation for the wild type full-length CaMKIIa ${ }^{23}$. The evidence for this came from molecular crowding experiments conducted with lysozyme, which increased the Hill coefficient for full-length CaMKIla wild type by $1.3-2$ fold, but not for full-length CaMKII containing an I321E mutation shown to disrupt the compact conformation. Under these molecular crowding conditions, the $\mathrm{EC}_{50}$ was reported to increase equally by $\sim 3$ fold for both wild type and I321E mutant, which was interpreted to indicate that the increase in the Hill coefficient (but not in the $\mathrm{EC}_{50}$ ) can be used as a readout of the compact conformation ${ }^{23}$.

A caveat to this previous study however, is that lysozyme has been shown to bind directly to $\mathrm{Ca}^{2+} / \mathrm{CaM}^{30}$, and therefore potentially complicates the interpretation of these results, especially regarding effects on the apparent $\mathrm{EC}_{50}$. Thus, we first compared the effects of molecular crowding by $150 \mathrm{mg} \mathrm{ml}^{-1}$ lysozyme and the putatively more inert molecule BSA (Fig. 5b,c and Supplementary Fig. 6). Consistent with lysozyme competition for $\mathrm{Ca}^{2+} \mathrm{CaM}$, crowding experiments performed with this reagent dramatically increased the

Figure 5 cont't. (d) Human CaMKIla and its I321E mutant that is incompetent for the compact conformation showed the same $\mathrm{Ca}^{2+} / \mathrm{CaM}$ responses during crowding with BSA as rodent CaMKIlla. (e, f) Linker-less (LL) CaMKII and its I321E mutant showed the same $\mathrm{Ca}^{2+} / \mathrm{CaM}$ response without crowding. However, crowding with BSA caused a lesser increase in $E_{50}$ for the I321E mutant that is incompetent for the compact conformation. The Hill slope was identical for both linker-less mutants and no longer showed significant cooperativity under crowding conditions. Error bars represent the standard error calculated from the curve fits. 
$E_{50}$, while crowding with BSA caused a significantly smaller effect (Fig. 5c). However, neither crowding condition increased the Hill coefficient; if any, it was slightly reduced (Fig. 5c). Thus, for full-length rodent CaMKIla wild type expressed in eukaryotic cells, the Hill coefficients in these in vitro crowding experiments did not provide any indication for a compact conformation.

Our initial experiments were conducted using rodent CaMKIla, as compared to the previous study that characterized the human isoform. Rodent and human CaMKIla differ only in one amino acid residue (asparagine versus serine at position 324 within the linker region). Nonetheless, we decided to test if this one amino acid difference could explain the different effect of molecular crowding on the Hill coefficient seen in our study with rodent CaMKII. However, the effects of molecular crowding with BSA on rodent versus human CaMKIla were indistinguishable (Fig. 5d and Supplementary Fig. 6). In order to test if the shift in $\mathrm{EC}_{50}$ that was caused by crowding with $\mathrm{BSA}$ could be a reflection of a compact conformation, this experiment additionally compared the effects on the I321E mutant that is designed to be incompetent for the compact conformation. Again, the effects on human CaMKII and its I321E mutant were indistinguishable (Fig. 5d and Supplementary Fig. 6), indicating that the observed shift in the $\mathrm{EC}_{50}$ did not reflect induction of a compact conformation for the full-length wild type CaMKII. These results further confirmed that full-length CaMKII exists largely in extended conformations, even under these conditions of molecular crowding. 
Finally, we tested the $\mathrm{Ca}^{2+} / \mathrm{CaM}$ response for a linker-less CaMKIla mutant (LL) that was described to be in the compact conformation even without crowding. We further compared this construct to a linker-less I321E combination mutant $(L L+\mid 321 E)$ that has been shown to remain in the extended conformation ${ }^{23}$. Surprisingly, without crowding, both mutants showed the same $\mathrm{Ca}^{2+} / \mathrm{CaM}$ sensitivity, both for activation and for T286 autophosphorylation (Fig. 5e,f and Supplementary Fig. 6). Compared to full-length CaMKII, a mild $\sim 1.5$ fold decrease in $\mathrm{Ca}^{2+} / \mathrm{CaM}$ sensitivity was observed (Supplementary Fig. 6). This effect is similar to the $\sim 2$ fold difference seen between two naturally occurring CaMKII $\beta$ variants with different linker lengths $\left(\beta\right.$ and $\beta \mathrm{e}^{\prime} ;{ }^{31}$ ), but not to the $\sim 500$ fold difference previously reported for the linker-less mutant after bacterial expression $^{23}$. Thus, after expression in mammalian cells for our kinase preparations, even the linker-less mutants were largely in an extended conformation, at least under non-crowding conditions. In contrast to full-length CaMKII, however, molecular crowding conditions differentially affected the linkerless kinase versus its I321E mutation that prevents the compact conformation. While crowding with $\mathrm{BSA}$ caused a shift in $\mathrm{EC}_{50}$ both for the linker-less kinase and its I321E mutant, this shift was significantly smaller for the I321E mutant (Fig. 5e,f). By contrast, the Hill coefficient was decreased to the same extent for both the linker-less kinase and its I321E mutant (Fig. 5e,f). These data show that the more extensive increase in the $\mathrm{EC}_{50}$ may provide a readout for the compact conformation for the linker-less kinase under molecular crowding conditions, while the change in Hill coefficient did not. More importantly, together these data 
show that after mammalian expression, crowding with BSA may induce a compact conformation for a linker-less mutant, but not for full-length CaMKII wild type. The numerical values for the effect on $\mathrm{EC}_{50}$ and Hill coefficient observed in our experiments are listed in (Supplementary Tables 1 and 2).

\section{The CaMKIla linker promotes an extended state in cells}

Compact versus extended CaMKII conformations result in different positioning of the kinase domains (see Fig. 5a), which would be expected to affect the FRET efficiency between labeled CaMKII subunits. Thus, we employed a FRET assay to evaluate a potential equilibrium between compact and extended CaMKII conformations by live cell imaging. Specifically, we compared full-length CaMKIla (WT) and the linker-less mutant (LL), with and without the I321E mutation that disrupts the compact conformation. Significant FRET was detected for all of these CaMKII forms, but not for a control CaMKII (1-316) that lacks the hub (association) domain and thus cannot form multimeric holoenzymes (Fig. 6 and Supplementary Fig. 7). Note that the control CaMKII shows significant localization in both the cytoplasm and in the nucleus, consistent with its monomeric nature that abolishes the nuclear exclusion seen for the larger holoenzymes (Fig. 6a). However, this does not affect the FRET results, as the FRET measurements were restricted to the cytosol and to cells with similar cytosolic FRET acceptor (mGFP) to donor (mCherry) ratio. Specifically, an acceptor excess of $4-11$ fold was selected in order to limit the analysis to cells in which the average holoenzyme (12-mer) contains at least one donor, but less 


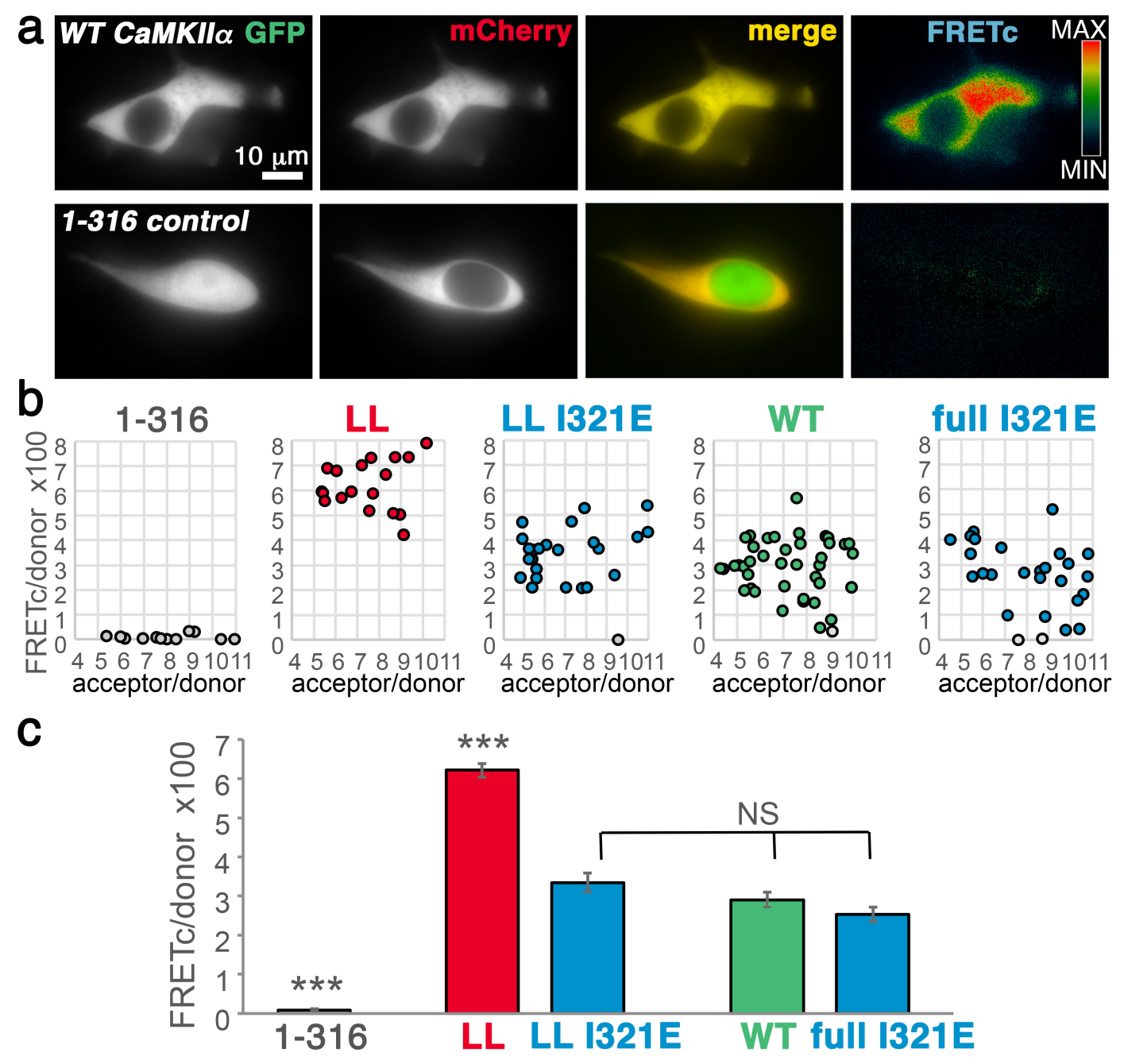

Figure 6: A FRET-based assay indicated a compact conformation within cells only for linker-less but not for full-length CaMKIlla wild type. (a) Coexpression of CaMKlla labeled with mCherry or mGFP (as FRET donor or acceptor) N-terminal of the kinase domain resulted in a significant FRET signal in HEK cells (FRETC; corrected for fluorescence bleed-through). Deletion of the hub domain in the GFP-labeled CaMKII (1-316; $n=12$ cells) abolished the FRET signal almost completely. Scale bar $=10 \mathrm{~mm}$. (b) FRETc was normalized by expression levels of the FRET donor (FRETc/donor) and plotted as function of the acceptor/donor ratio (within a range of $4-11$ fold acceptor access). The few cells that showed complete FRET failure (grey) remained included in the analysis. (c) The linker-less (LL) CaMKIla mutant ( $n=17$ cells) showed significantly higher FRET than all other constructs $\left(^{* * *}: p<0.001\right.$; ANOVA with Tukeys posthoc analysis). FRET of full-length CaMKlla wild type (WT; $n=38$ ), of its I321E mutant (full I321E; $n=26$ ) that is incompetent for the compact conformation, and of a linker-less I321E mutant (LL I321E; $n=22$ ) were indistinguishable (NS: $p>0.05)$. Error bars represent the standard error of mean (s.e.m.). 
than 3 donors (Fig. 6b). For the linker-less CaMKII, FRET was significantly reduced by the additional I321E mutation (Fig. 6c). This result is consistent with a compact conformation of the linker-less CaMKII that is disrupted by the mutation, and thus indicates that a compact conformation can be detected by this FRET assay within cells. In contrast to the linker-less mutant, full-length CaMKII wild type showed FRET that was unaffected by the I321E mutation (Fig. 6c). Thus, these data support the conclusion that full-length CaMKIla wild type exists largely in extended conformations within cells.

\section{Discussion}

The intrinsic dynamics within the CaMKII holoenzyme architecture identified here has confounded previous attempts at obtaining a complete structural description of CaMKII. However, it is this intrinsically dynamic behavior of CaMKII that enables the complex regulation that leads to molecular memory formation. Single particle EM is ideally suited for studying such dynamic systems because images of individual particles are obtained with extraordinarily high-resolution, and computational classification methods may effectively deconvolute inherent conformational heterogeneity. Using this approach, we have described (i) a pseudo-atomic model of the dodecameric CaMKII holoenzyme in its activationcompetent extended conformation, (ii) a high flexibility of kinase domain positioning for this predominant extended state, and (iii) an equilibrium with other conformational states that are activation-incompetent (Fig. 7). The predominant form is organized by the central dodecameric hub complex, with each of the 
twelve kinase domains tethered at peripheral positions by a flexible internal linker (the variable linker region). Two activation-incompetent states were identified as minor populations and included kinase domain dimers $(<20 \%)$ and a compact conformation (<3\%). An additional minor population was found with holoenzymes assembled as 14-mers (< $4 \%)$ instead of 12-mers (Supplementary Fig. 3). As discussed below, these equilibria shape the regulation of CaMKII activation and may enable subunit exchange between holoenzymes.

Our 3D EM reconstruction shows a CaMKII holoenzyme in an extended activation-competent conformation at pseudo-atomic resolution, with each of the major domain components being well defined by high-resolution crystallographic structures. It should be noted that previous attempts at 3D CryoEM reconstructions have been made; however, the results were inconsistent with Xray crystallographic structures of the organizing hub complex ${ }^{32,33}$, likely due to complications associated with particle orientation preference that were described in these studies and observed in our own work as well. For our work, complications with specimen orientation preferences were overcome by applying tomographic tilted-imaging routines, which resulted in a 3D reconstruction that matches well with individual particle images (Supplementary Fig. 5) and to previous X-ray crystallographic structures of the isolated hub and kinase domain (Figure 2). However, while the hub domains in our reconstruction occupy a very defined volume, our results also show that the kinase domain positioning is highly flexible. Consequently, only a minor fraction of holoenzymes would be in 


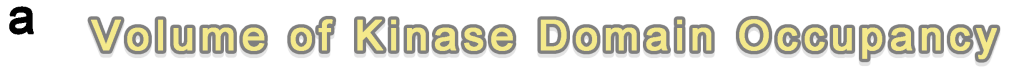

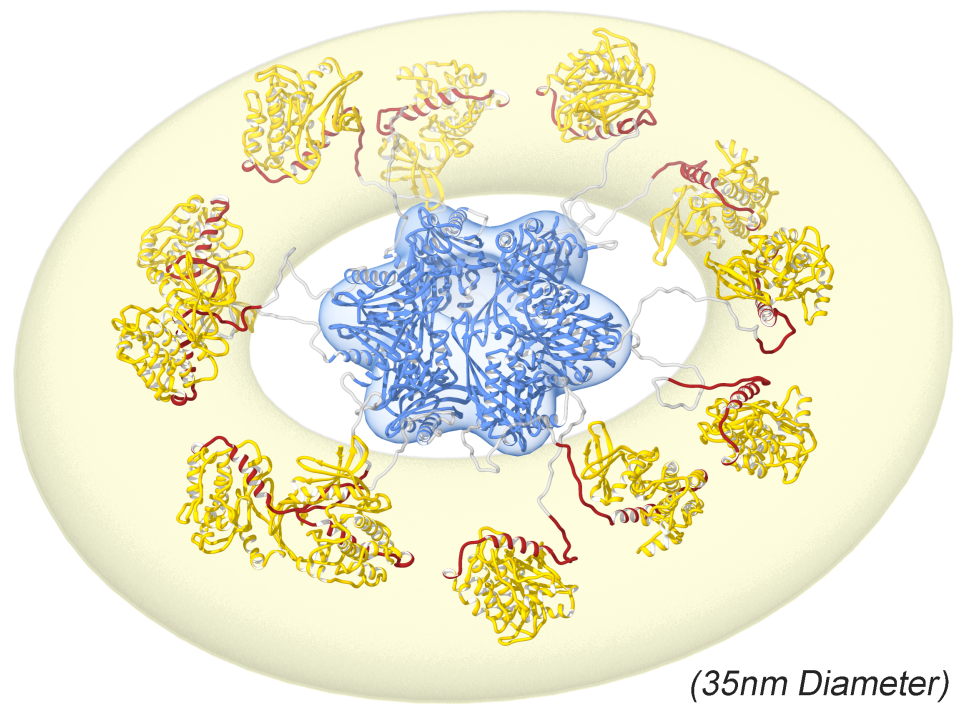

b

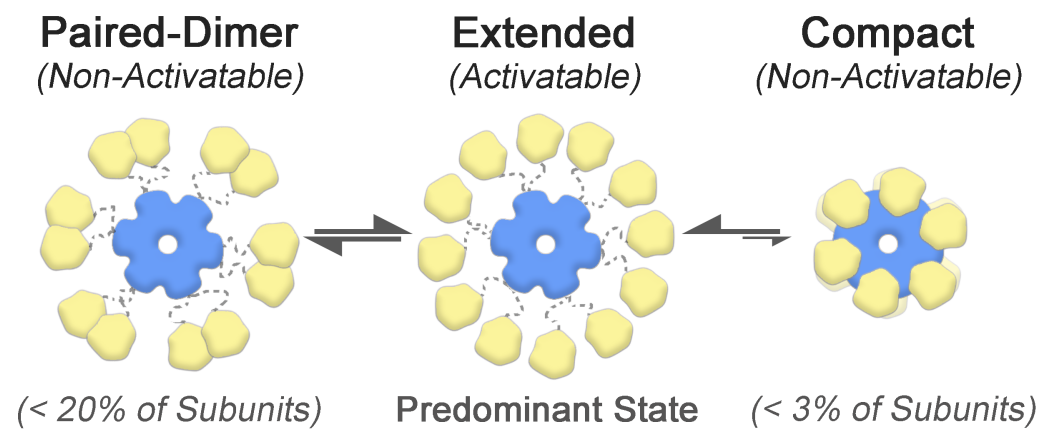

Figure 7: Overview of CaMKIla kinase domain arrangements. (a) Illustration of the CaMKII holoenzyme indicating an overall volume of kinase domain occupancy observed by single particle EM (yellow halo). Flexible linker regions support a continuum of kinase domain arrangements within this volume of occupancy (maximum diameter of $\sim 35 \mathrm{~nm}$ ). (b) Three major conformational states appear to exist in equilibrium. A predominant extended and activatable state, as depicted in panel $\mathbf{a}$, is distinguished by an extended conformation with non-interacting kinase domains. Additional non-activatable states are distinguished by the presence of dimeric pairing between neighboring kinase domains (representing less than $20 \%$ of subunits), as well as a putative compact form distinguished by kinase-hub domain interactions (representing less than 3\% of subunits). For clarity, fully paired and fully compact states are illustrated in panel b. However, conformational states where all twelve kinase domains are simultaneously paired appear to be rare ( 2.5\% of structures) and the fully compact state with all kinase subunits of an individual holoenzyme in the compact conformation was not observed at all for full-length CaMKII holoenzymes by EM or by live cell FRET analysis. 
the actual conformation of this average structure. While the average structure marks the preferred positioning for an individual kinase domain, the degree of this preference is so small that it is highly unlikely for all kinase domains of a holoenzyme to occupy this average position at the same time. As a consequence of this kinase domain flexibility, individual holoenzyme particle sizes can vary in diameter from $\sim 15$ to $35 \mathrm{~nm}$.

It was clear that kinase domain flexibility must exist in order to enable the intersubunit autophosphorylation at T286 that generates autonomous CaMKII activity and is required for long-term synaptic plasticity. However, it was unclear if such flexibility needed to be induced by $\mathrm{Ca}^{2+} / \mathrm{CaM}$ stimulation, or if it is already an intrinsic property of the holoenzyme in its basal state, as shown here. The presence of such dynamic behavior even in the basal-state suggests an additional functional role, which we propose to be involved in shaping the activation properties of the holoenzyme.

It has been previously proposed that the cooperative activation profile of CaMKII is a result of a dynamic equilibrium between an activatable extended state and a non-activatable compact state $^{23}$. In this model, CaM binding to one subunit promotes the activatable state of neighboring subunits, leading to cooperative activation, which in turn would shape the frequency-dependent response of CaMKII to $\mathrm{Ca}^{2+}$-oscillations. Indeed, we found CaMKII activation with Hill Coefficients of $1.5-2$. This is consistent with previous reports, and indicates that 
activation by $\mathrm{Ca}^{2+} / \mathrm{CaM}$ is a cooperative process ${ }^{20,32,34}$. However, the compact conformation constituted only a very small fraction of eukaryotically expressed CaMKII holoenzymes, even under molecular crowding conditions (in vitro or within cells). Indication for a compact conformation was found only for a linkerless CaMKII mutant, but not for full-length (wildtype) CaMKIla holoenzymes. Thus, we conclude that cooperativity of CaMKII activation must be based on additional mechanisms.

Our data suggest cooperativity of CaMKII activation is enabled by an equilibrium with an additional activation-incompetent conformation that involves kinase domain dimerization via their $\mathrm{Ca}^{2+} / \mathrm{CaM}$ binding regulatory domains. Our results indicate that such dimeric-pairs may represent as much as $20 \%$ of the total subunit population; within single holoenzymes a variety of paired and unpaired arrangements appear to be present. For isolated kinase domains, such dimerization has been captured in a crystal structure of $C$. elegans CaMKII ${ }^{19}$ and has been reported to occur with low affinity for human CaMKII $\left(K_{d} 200-600\right.$ $m M)^{21}$. While this is an extremely low affinity for a specific protein-protein interaction, the local kinase domain concentration in context of the holoenzyme structure determined here is estimated to be $\sim 3 \mathrm{mM}$ (see Methods). Under such high local concentrations, both paired and unpaired states would be significantly populated under equilibrium conditions, as observed here. In addition to affinity and concentration, the extent of kinase domain dimerization would be governed by the freedom of movement of the kinase domains, which is provided by the 
variable linker domain. Thus, deletion of the linker domain would be expected to impair kinase domain dimerization, which in turn should reduce cooperativity of activation. Consistent with this notion, cooperativity was found to be reduced in the linker-less CaMKII (Figure 5 and Supplementary Tables 1 and 2). Under molecular crowding conditions, the Hill Coefficient for the linker-less CaMKII approached 1 , indicating that cooperativity was almost completely abolished. Taken together, CaMKII holoenzymes exist in an equilibrium between a predominant extended, flexible, activation-competent state and two distinct and more restricted activation-incompetent states (Fig. 7). One of the activationincompetent states is characterized by kinase domain dimerization and the other by kinase domain binding to the association domain that result in a compact conformation. However, the compact conformation constitutes only a very minor fraction and therefore does not significantly contribute to the cooperative CaMKII activation characteristics.

Biochemical and EM studies have established a general consensus that the CaMKII holoenzyme is formed by 12 subunits ${ }^{33,35,36}$. However, this was recently called into question by a study that reported a $\sim 1: 1$ ratio of 12-meric to 14-meric holoenzymes ${ }^{22}$. By contrast, while our study detected a small fraction of 14-meric particles $(<4 \%)$, the vast majority of holoenzymes were 12-meric (Supplementary Fig. 3). Previously, it was thought that truncated association domains form 14-mers with 7-fold symmetry while full-length subunits form 12mers with 6-fold symmetry ${ }^{37}$. However, the majority of 14 -meric particles in our 
study were associated with 14 kinase domains, demonstrating that 14-mers can indeed be formed by full-length subunits. By contrast, the higher extent of 14mers in this other recent study could potentially be due to partial loss of kinase domains. Alternatively or in addition, the difference may be due to bacterial expression versus eukaryotic expression in our study. Importantly, while eukaryotic CaMKII holoenzymes are largely 12-meric, the 14-meric holoenzymes may provide a transition state that allows exchange of subunits. As stated above, it has been shown that proteolytic cleavage of the kinase domains from a 12meric holoenzyme preparation results in the subsequent formation of 14-meric hub domain assemblies ${ }^{37}$. Therefore, while CaMKII wildtype is exclusively observed in holoenzymes, an exchange of subunits is possible. This mechanism may allow the exchange of damaged subunits without necessitating that the entire holoenzyme be discarded. Other more speculative functions of subunit exchange have also been suggested; as such exchange can be promoted not only by kinase domain deletion, but also by stimulation ${ }^{38}$. These studies have used FRET-based approaches to examine subunit exchange, and the transition states are not resolved. As in the damage-induced exchange process, these transition states may be 14-meric, and our study provides direct evidence that intact full-length CaMKII holoenzyme can indeed form such 14-mers.

It remains unclear what other functional roles are enabled by the dynamic behavior of CaMKII holoenzymes. For example, how are the various populations of conformational states affected by $\mathrm{Ca}^{2+} / \mathrm{CaM}$ stimulation, and what organization 
takes place between local kinase domains to facilitate inter-subunit transactivation and transition to autonomously active particles. Previous FRET studies suggest additional reorganization or expansion of the holoenzyme upon stimulation by $\mathrm{Ca}^{2+} / \mathrm{CaM}^{29,39,40}$. This observation is consistent with our basal-state model and the expected release of the auto-inhibitory domain upon activation, which should enable a further increase the distance between the kinase and hub domains. However, it is unclear how these induced conformational changes may influence the overall dynamic behavior of the signaling particle. Furthermore, it is uncertain how the holoenzyme architecture enhances binding to GluN2B, enabling molecular memory formation in this context ${ }^{6,9,10}$. Future experiments aimed at addressing these questions are almost certain to provide new insights into the enigmatic mechanism of molecular memory formation, and unveil more surprising features of this remarkable signaling complex.

\section{Methods}

\section{CaMKII and CaM preparations}

Rat CaMKIla wild type was purified after baculovirus/Sf9 cell expression and CaM was purified after bacterial expression. Briefly, CaM was purified by differential ammonium sulfate precipitation followed by phenyl-sepharose columns $^{41}$. CaMKII was purified from cytoplasmic $100,000 \mathrm{~g}$ supernatants via two sequential column purification steps, a P11 phospho-cellulose column followed by a CaM-sepharose affinity column ${ }^{42,43}$. 
Human CaMKIla and CaMKIla mutants were expressed in HEK-293 cells. The cells were grown to $\sim 50 \%$ confluence in $10 \mathrm{~cm}$ dishes, then transfected using the $\mathrm{Ca}^{2+}$-phosphate method ${ }^{44,45}$. After two days of expression, HEK cells were homogenized with a motorized pellet pestle (Kontes) for $10 \mathrm{~s}$ in $0.4 \mathrm{ml}$ of ice-cold $50 \mathrm{mM}$ PIPES pH 7.2, 10\% glycerol, $1 \mathrm{mM}$ EDTA, 1 mM DTT, and complete protease inhibitor (Roche), then centrifuged at $16,000 \times g$ for $20 \mathrm{~min}$. The CaMKII concentration in the resulting supernatant was determined by quantitative western blot using a standard curve containing $250-1250$ fmole of purified recombinant CaMKIla diluted in non-transfected HEK cell extract. Blots were probed for CaMKIla expression using CBa2 antibody (1:2000, produced inhouse).

\section{CaMKII activity assay}

CaMKII activity was measured by ${ }^{32} \mathrm{P}$ incorporation into the peptide substrate Syntide-2. Reactions were started by adding purified CaMKIla or HEK cell extract containing CaMKIla to a final concentration of $2.5 \mathrm{nM}$ in a mix of $50 \mathrm{mM}$ PIPES $\mathrm{pH} 7.2,0.1 \%$ BSA, $2 \mathrm{mM} \mathrm{CaCl} 2,10 \mathrm{mM} \mathrm{MgCl}, 100 \mathrm{mM}\left[\mathrm{r}^{-32} \mathrm{P}\right] \mathrm{ATP}(\sim 1 \mathrm{Ci}$ mmole $^{-1}$ ) $1 \mu \mathrm{M}$ microcystine-LR, $75 \mathrm{mM}$ Syntide-2 and $3 \mathrm{nM}$ to $20 \mu \mathrm{M}$ calmodulin ${ }^{42,43}$. Some reactions additionally contained $150 \mathrm{mg} \mathrm{ml}^{-1}$ BSA or lysozyme, as indicated. Mixtures $(50 \mu \mathrm{l})$ were reacted for 3 minutes at $30^{\circ} \mathrm{C}$. Reactions were stopped by adding $15 \mu$ of ice cold $15 \%$ TCA, vortexing, and incubating on ice for $20 \mathrm{~min}$. Reactions were then centrifuged at $16,000 \mathrm{Xg}$ for 20 min to remove precipitated protein. $35 \mu \mathrm{l}$ of the supernatant containing the 
peptide substrate was spotted onto P81 cation exchange chromatography paper (Whatman) squares. After extensive washes with water, phosphorylation of the substrate peptide bound to the P81 paper was measured by liquid scintillation counting. Results were plotted using Graph Pad Prism 5 and fit using a nonlinear regression with variable slope.

\section{CaMKII autophosphorylation assay}

To assess the ability of CaMKIla WT and mutants to autophosphorylate at T286, the kinases were added at a final concentration of $20 \mathrm{nM}$ to a reaction buffer containing $50 \mathrm{mM}$ PIPES pH 7.2, 0.1\% BSA, $2 \mathrm{mM} \mathrm{CaCl} 2,10 \mathrm{mM} \mathrm{MgCl}_{2}, 100$ mM ATP, $1 \mu \mathrm{M}$ microcystin-LR, 100nM calmodulin. Kinases were reacted at $30^{\circ} \mathrm{C}$ for 30 seconds, or as indicated. Reactions were stopped by addition of gel loading buffer (2\% SDS, $50 \mathrm{mM}$ DTT, $67.5 \mathrm{mM}$ Tris $\mathrm{pH}$ 6.8, 10\% glycerol, 0.16 $\mathrm{mg} \mathrm{ml}^{-1}$ bromophenol blue) and boiling for $5 \mathrm{~min}$. Samples were loaded on $10 \%$ SDS-PAGE gels then transferred to PVDF membranes ${ }^{42,43}$. Blots were blocked in 5\% milk then probed with anti-phospho-T286 CaMKII antibody (Phosphosoutions) diluted 1:3000 in 1\% milk. Images were acquired on an Alpha Imager (Alpha Innotech) after exposure to Western Lightning ECL reagent (Perkin Elmer) and quantified ${ }^{42-44}$.

\section{FRET microscopy and image analysis}

HEK-293 cells (authenticated by STR analysis and tested for mycoplams) were grown to $50 \%$ confluency on glass coverslips and then transfected using the 
$\mathrm{Ca}^{2+} /$ phosphate method, using a 4:1 ratio of mCherry over GFP constructs. $24 \mathrm{~h}$ after transfection, images of live cells were acquired at $32^{\circ} \mathrm{C}$ on a climate controlled Zeiss Axiovert 200M microscope (Carl Zeiss GmbH, Oberkochen, Germany) at $100 \mathrm{X}$ magnification using slide book 5.5 software (Intelligent Imaging Innovations) in imaging solution containing $0.87 \times$ HBSS, $25 \mathrm{mM}$ HEPES, $2 \mathrm{mM}$ Glucose, $2 \mathrm{mM} \mathrm{CaCl} 2,1 \mathrm{mM} \mathrm{MgCl}$. FRET image acquisition and analysis were done by the three-filter "micro-FRET" image subtraction method" In brief, three single plane images (40-ms to $500-\mathrm{ms}$ exposure sets, $2 \times 2$ binning) were obtained: a GFP excitation/GFP emission image; a mCherry excitation/mCherry emission image; and a GFP excitation/mCherry emission image (raw, uncorrected FRET). Background-subtracted GFP and mCherry images were then fractionally subtracted from raw FRET images based on measurements for GFP bleedthrough (0.02093 fraction of GFP image) and mCherry cross-excitation ( 0.09484 fraction of mCherry image). This fractional subtraction generated corrected $\mathrm{FRET}_{\mathrm{C}}$ images, represented in pseudo-color. The fractional subtraction coefficients are rounded up from average cross-bleed values determined in cells expressing GFP- or mCherry-tagged constructs alone. Thus, these coefficients result in a slight underestimation of $F R E T_{C}$ signals for true FRET partners but limit false positive detection of FRET.

Absolute FRET $\mathrm{C}$ values depend both on FRET efficiency and on the amount of fluorophores present. Thus, in order to compare FRET among different cells, the $\mathrm{FRET}_{C}$ values were divided by the intensity of the donor fluorophore. This simple normalization method is valid when the acceptor is in excess; here, only cells 
with 4-11fold acceptor excess were included in the analysis. Acceptor/donor ratio was determined based on the detected GFP/mCherry signal combined with the 4.8 more efficient fluorescence detection of GFP in our setup (as determined in cells expressing a GFP-mCherry fusion protein). Image acquisition was done based on the GFP and mCherry channels, i.e. blind of the raw FRET channel. Image analysis was performed blind of the condition.

\section{CaMKII preparation for electron microscopy}

Full-length CaMKIla purified from eukaryotic Sf9 cell expression was prepared for negative stain EM by diluting the purified protein $\left(1: 40 \mathrm{vol} \mathrm{vol}^{-1}\right)$ in $\mathrm{EM}$ buffer containing $50 \mathrm{mM}$ HEPES (pH 7.4), $120 \mathrm{mM} \mathrm{KCl}$ and $0.5 \mathrm{mM}$ EGTA. A $3 \mathrm{ml}$ drop of sample ( 100 nM) was applied to a glow-discharged continuous carbon coated EM specimen grid (Ted Pella). Excess protein was removed by blotting with filter paper, and washing the grid two times with EM buffer. The specimen was then

stained with freshly prepared $0.75 \%\left(\mathrm{wt} \mathrm{vol}^{-1}\right)$ uranyl formate (SPI-Chem). Cryogenically prepared specimens were prepared by applying a $3 \mathrm{ml}$ drop of sample $(\sim 1 \mathrm{mM})$ to a negatively charged Quantifoil holey carbon specimen grid (Electron Microscopy Science). The sample was blotted with filter paper and plunged into liquid ethane using a vitrobot (FEI) and stored under liquid nitrogen.

\section{Electron microscopy and image processing}

Negatively stained specimens were visualized on a $120 \mathrm{kV}$ TEM (iCorr, FEI) at a nominal magnification of $49,000 x$ at the specimen level. Digital micrographs were 
recorded on a $2 \mathrm{~K} \times 2 \mathrm{~K} \mathrm{CCD}$ camera (FEl Eagle) with a calibrated pixel size of $4.37 \AA$ pixel $^{-1}$ and a defocus of $1.5-2.5 \mathrm{~mm}$. To overcome issues with particle orientation preference on the EM grid, serial tomographic images were collected at tilts angles of $0^{\circ}-50^{\circ}\left(\Delta 10^{\circ}\right)(\text { Supplementary Fig. 5 })^{47}$. CTF parameters were determined in EMAN2 ${ }^{48}$ and micrographs free of significant astigmatism and drift were selected based on Thon rings in the power spectra. A total of 16,616 particles were hand selected in EMAN2 (10902 un-tilted and 5,714 tilted particles) and extracted with a box size of $128 \times 128$ pixels. Reference-free 2D class averages were generated in EMAN2 ${ }^{48}$ and RELION v1.4 $4^{49}$ using CTFcorrected (phase-flipped) and band-pass filtered images without any applied symmetry. 2D variance maps were calculated in RELION by squaring the standard deviation of aligned particle images present in the 2D class average.

To separate coexisting conformational states of the CaMKIla hub domain (dodecamer or tetradecamer), a focused reference-free 2D classification was preformed in RELION using a subset of $\sim 8,000$ un-tilted particles with a soft outer mask (75 $\AA$ radius) applied prior to classification. A set of 80 classes was produced and analyzed to determine the relative populations of hub domains comprising of six-fold and seven-fold symmetry (Supplementary Fig. 3). The constituent particles from classes with apparent seven-fold symmetry were extracted as unmasked images, and reclassified for validation. For analysis of the kinase domains, a soft inner mask (50 $\AA$ radius) was applied to remove densities corresponding to the hub domain prior to 2D classification. 
Cryogenically prepared CaMKIla particles were imaged on a 300kV Titan Krios (FEI). Digital micrographs were recorded on a Falcon II direct electron detector (FEI) using low-dose imaging routines at a nominal magnification of 47,000x and defocus of $3-5 \mathrm{~mm}$. CTF correction and image processing routines were carried out in EMAN2, as described above. 2D class averages were obtained from a small dataset of $\sim 500$ individual particle images.

\section{Single particle measurements and statistical analysis}

Statistical analyses of individual particle dimensions were obtained by measuring particle lengths on un-binned micrographs using the measurement tool in EMAN2. A random set of 82 representative un-tilted individual particle images was inspected. A radius of extension for individual kinase subunits $(n=928)$ was obtained by measuring the distance from the center of the pore in the hub domain complex to the center of each peripheral density corresponding to the kinase domains. For each of these measurements, a distance of $22.5 \AA$ was appended (corresponding to the average radius of the kinase domain) to yield a value that represents the full extension of the kinase domain. Inter-kinase domain separation distances were determined by measuring from the center of one peripheral kinase domain density to the center of the closest neighboring density in the clockwise direction. Only densities that could be clearly resolved as individual kinase domains were incorporated into this analysis $(n=883)$. The

distribution of individual particle measurements were binned into $5 \AA$ increments for histogram analysis (corresponding approximately to the pixel value of these 
images $=4.37 \AA$ ). A standard Gaussian curve was fit to the distribution using the experimentally determined mean and standard deviation. Whisker plots were generated to represent the minimum, maximum and $25 \%, 50 \%$, and $75 \%$ quartile values for each dataset. A correlation map was obtained by scatter plot analysis of kinase radius versus kinase domain separation. All statistical analyses were performed in Microsoft Excel.

\section{D reconstruction and refinement}

An initial three-dimensional (3D) reconstruction was determined using EMAN2 from a culled subset of tilted and un-tilted particles showing clearly defined hub domains and separated kinase domains in 2D class averages. A calculated map for the isolated human CaMKIla hub domain (PDBID $51 \mathrm{G}^{2} ;{ }^{22}$ ) was filtered to 40 $\AA$ and used as a search model for initial alignments. This produced a $\sim 25 \AA$ resolution reconstruction with imposed D6 symmetry displaying strong density for the central hub domain and weak peripheral densities representing the twelve kinase domains assembled by the dodecameric holoenzyme (Supplementary Fig. 4). This initial reconstruction was filtered to $60 \AA$ and used as a reference for masked 3D refinements in RELION.

Separate refinements of the hub domain and kinase domains were performed in RELION by incorporating a 3D Gaussian mask based on the central hub feature produced by the initial 3D reconstruction in EMAN (Supplementary Fig. 4). For the hub-only refinement, the mask was set to remove peripheral densities corresponding to the kinase domains during the alignment procedure (max 
diameter $=\sim 125 \AA$ ). For the kinase domain refinement, the 3D mask was extended by $22 \AA$ and inverted to remove density corresponding to the central hub domain during alignment. Both refinements proceeded with applied D6 symmetry. The hub-only refinement was determined to a resolution of $\sim 19 \AA$ (gold standard FSC). For the kinase domain refinement, a subset of images was obtained from a combination of $2 \mathrm{D}$ and $3 \mathrm{D}$ classification performed in RELION. For 3D classification, six classes were generated with D6 symmetry imposed. Four of the six classes had clearly resolved kinase domains and the particles in these classes were selected for further refinement, resulting in a final resolution of $\sim 20 \AA$ (gold standard FSC). These two maps were then combined using a spherical mask to remove overlapping densities (radius $=75 \AA$ ). A final combined density map representing the full-length holoenzyme has been deposited to the EM Data Bank (EMD-8514).

\section{Molecular modeling of the human CaMKIla holoenzyme}

A pseudo-atomic model of the full-length CaMKIla holoenzyme was constructed by fitting the atomic coordinates of the dodecameric human CaMKIla hub domain (PDBID 5IG3;22 residues 345 - 472) and monomeric human CaMKIla kinase/regulatory domain (PDBID $2 \mathrm{VZ6},{ }^{21}$ residues $13-300$ ) into the EM density map by rigid body fitting using UCSF Chimera ${ }^{50}$. The orientations of the kinase domains were chosen based on best fit to the map. Two neighboring kinase domains were fit separately and symmetrized to reflect the six-fold dihedral symmetry in the EM map. The fitted domains gave good agreement to the 
experimental density (cross-correlation at $20 \AA$ resolution $=0.95$ for the hub complex and 0.98 for kinase domains). A flexible linker connecting these two structured domains (residues 301 - 344, human sequence), including the distal region of the CaM binding site (residues 301 - 314) and variable linker region (residues 315 - 344). Linkers were constructed separately for each chain using the MODELER loop building tool $^{25}$, and subjected to steepest descent minimization and subsequent conjugate gradient minimization routines in Chimera to regularize the geometries and remove steric interactions. Atomic coordinates for the constructed model have been deposited with the Protein Data Bank (PDBID 5U6Y).

A model for calculating the local concentration of kinase domains within the CaMKII holoenzyme was based on calculating the volume of a torus (Volume $=$ $\left.\left(\pi r^{2}\right) \times(2 \pi R)\right)$; where $R$ and $r$ represent the major and minor radius of the torus, respectively), as defined by the minimum and maximum kinase radius of extension determined in this work (providing $R=126 \AA$ and $r=49 \AA$ ). This volume, representing the space occupied by kinase domains surrounding the central hub complex, was then used to estimate the local concentration of kinase domains for a dodecameric holoenzyme, resulting in $\sim 3.3 \mathrm{mM}$, which corresponds well to previously determined estimates ${ }^{22}$.

Protein structures were visualized and images captured in UCSF Chimera. Figures were prepared in Adobe Photoshop. The contrast of EM micrographs 
and 2D class averages were similarly adjusted for manuscript presentation.

\section{Data Availability}

The EM map has been deposited with the EM database (EMD-8514). The pseudo-atomic model of the CaMKII holoenzyme fit to the EM density has been deposited with the Protein Data Bank (PDBID 5U6Y).

\section{Acknowledgements}

We are grateful for the assistance in electron microscopy provided by Claudia Lopez at the Multiscale Microscopy Core (OHSU, FEI Living Lab). This work was supported by start-up funds and Faculty Enhancement Grant from PSU (S.L.R.), and by NIH R01NS081248 (K.U.B.).

\section{Author Contributions}

K.U.B. and S.L.R. designed the project. JM performed the electron microscopy (EM) and image processing. A.M. contributed to EM image processing and statistical analysis. V.Z. performed molecular cloning and FRET studies, S.J.C. performed sample preparation for EM and in vitro biochemistry. K.U.B. and S.L.R. wrote the manuscript with contributions from all of the authors on this work.

\section{Competing financial interests}

K.U.B. is owner of Neurexus Therapeutics. 


\section{Supplementary Figures and Legends}

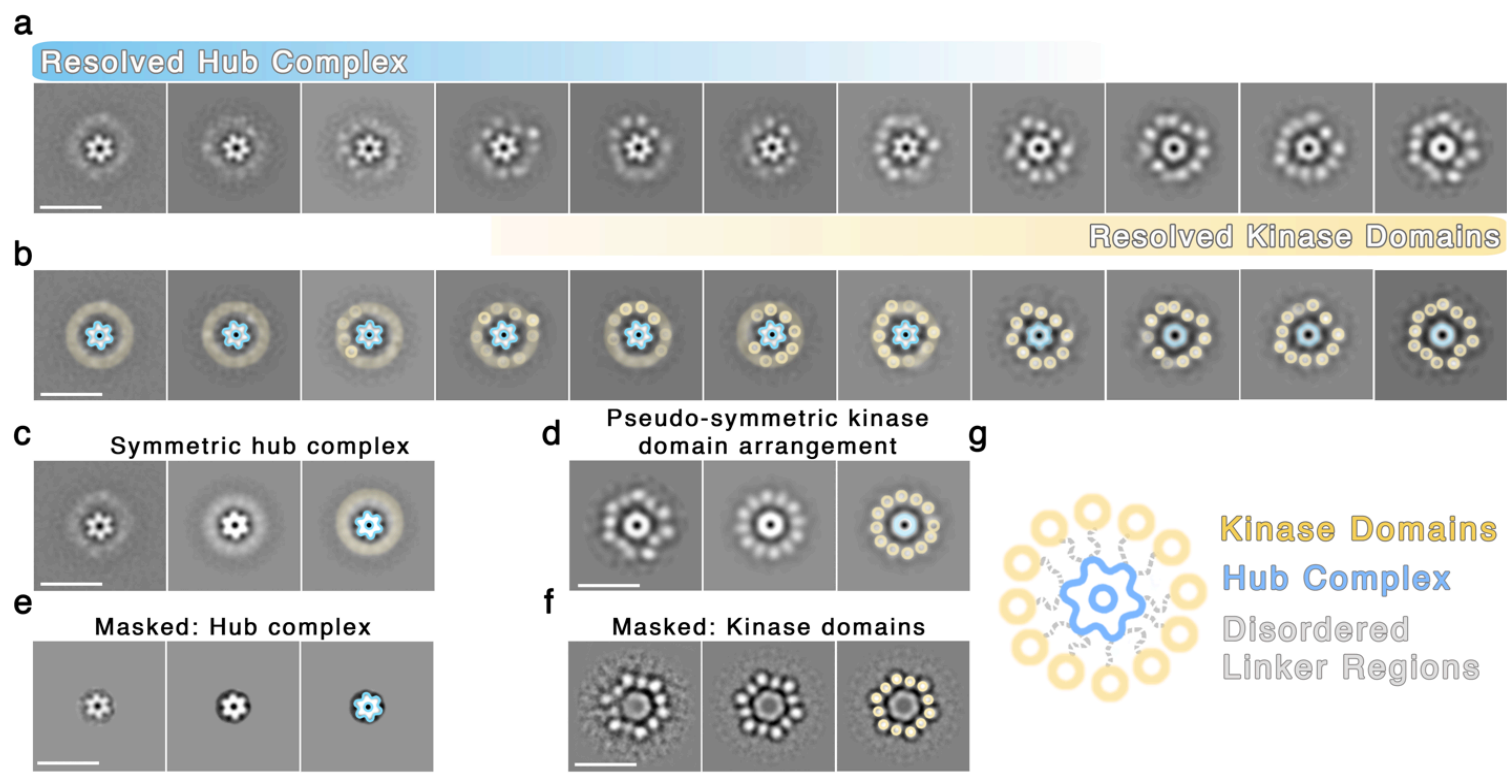

Supplementary Figure 1: Overview of 2D classification routines and analysis. (a) Reference-free 2D class averages obtained from unmasked images result in a continuum of structures, displaying either a well-resolved hub complex (left) or up to twelve resolved kinase domains (right). Scale bar in all panels $=25 \mathrm{~nm}$. (b) Same as panel a, with resolved hub complex (blue outline) and kinase domains (yellow circles) indicated. Faded circles (yellow and blue) indicate regions of unresolved structure. (c) Representative class average with a well-resolved hub complex (left), and with applied 6-fold rotational symmetry (middle and right). (d) Representative class average with twelve resolved kinase domains (left), with applied 6-fold rotational symmetry (middle and right). (e) Representative class average obtained with an applied $75 \AA$ outer radius mask to remove contributions of the kinase domain during the alignment procedure (left), and with applied 6-fold rotational symmetry (middle and right). (f) Representative class average obtained from particle images with an applied $50 \AA$ inner radius mask to remove contributions of the hub domain (left), and with applied 6-fold rotational symmetry (middle and right). Symmetry was only applied after 2D classification results. (g) Illustration of domain positions observed in symmetrized class averages from 


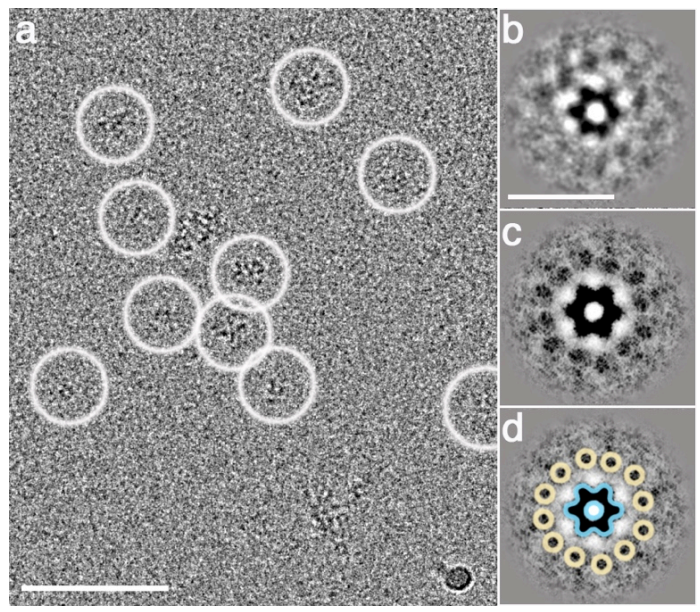

Supplementary Figure 2: Vitrified CaMKIla particles imaged by electron cryo-microscopy. (a) Electron micrograph obtained from cryogenically prepared CaMKIla holoenzymes (contrast of protein is dark on lighter background). Isolated single particles are indicated by white circles. Scale bar $=100 \mathrm{~nm}$. (b) 2D class average obtained from a small test dataset of $\sim 500$ particles indicates that frozen hydrated CaMKIla particles adopt structures similar to those observed by negative stain EM. Scale bar $=25 \mathrm{~nm}$. (c, d) Class average in panel $\mathbf{b}$ with applied 6-fold rotational symmetry enhances the contrast and pseudo-symmetric arrangement of up to twelve kinase domain densities and the 6-fold symmetric hub domain complex (yellow circles and blue outline in panel $\boldsymbol{d}$, respectively). 
a

Focused 2D Classification of Central Hub Complex

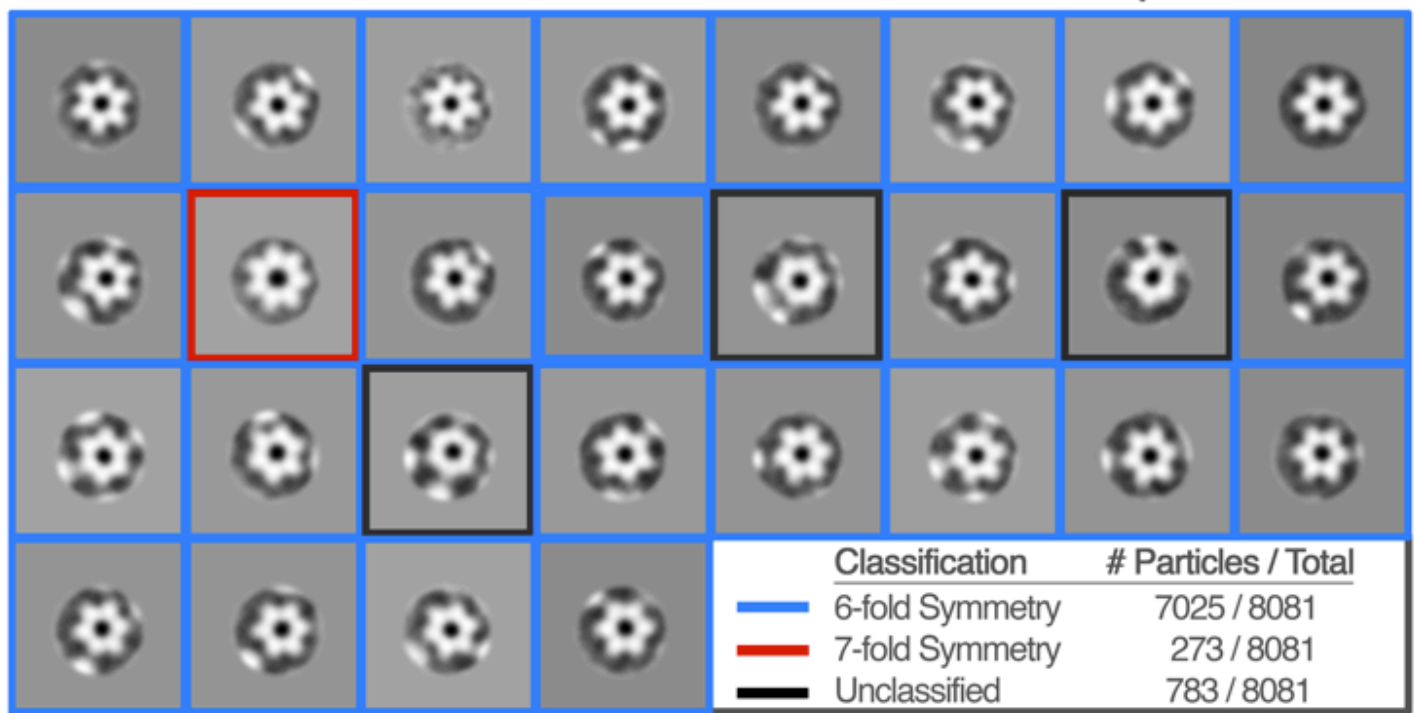

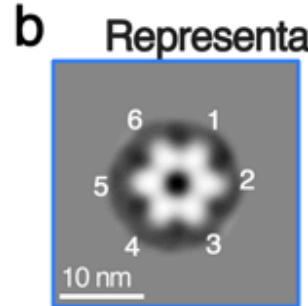

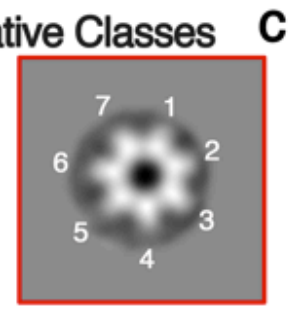

C

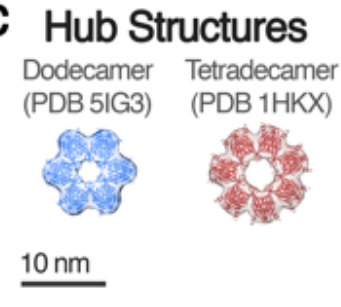

e d Class Distribution

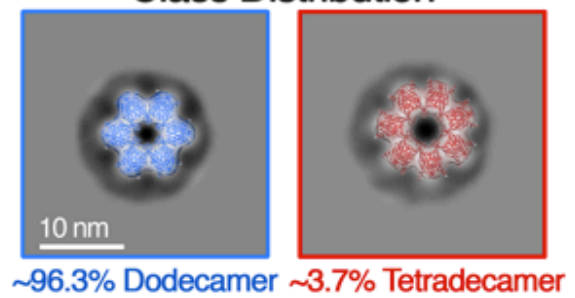

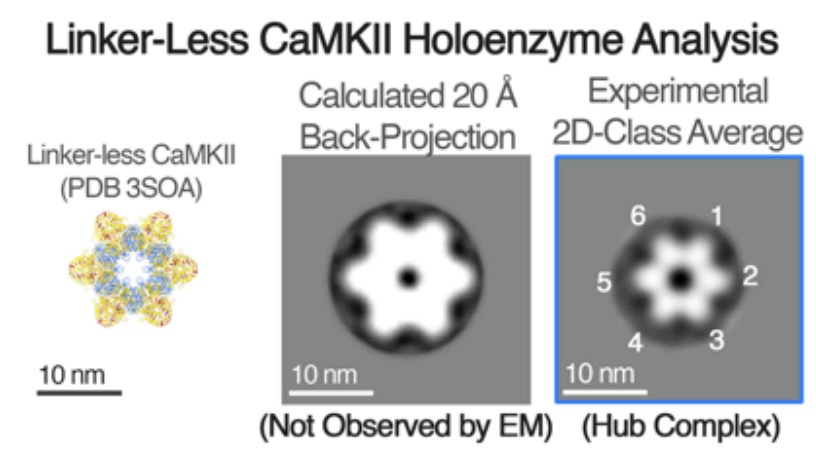




\begin{abstract}
Supplementary Figure 3: Focused 2D classification of hub complex structures observed by negative stain EM. (a) Focused 2D classification results of the hub domain complex were obtained using an image mask ( $75 \AA$ outer radius) that was applied to remove contributions of the kinase domains during the alignment procedure (28 of a total 80 classes are displayed). No symmetry was applied during classification and alignment. Class averages were categorized as displaying 6-fold symmetry (blue outline), 7-fold symmetry (red outline), or unclassified (black outline). Inset, Indicates the population of particles belonging to each category. (b) Representative class average of hub domain complexes with identified 6-fold symmetry (left) and 7-fold symmetry (right). Scale bar in all panels $=10 \mathrm{~nm}$. (c) Crystallographic structures reported for dodecameric (blue ribbon, PDBID $5 \mathrm{IG}^{22}$ ) and tetradecameric (red ribbon, PDBID $1 \mathrm{HKX}^{18}$ ) assemblies of the isolated hub complex. (d) Crystal structures in panel $\mathbf{c}$ fit into the EM densities in panel b. The two models fit with good agreement to their respective $2 \mathrm{D}$ class averages. The percentage of classified hub complexes identified as dodecameric and tetradecameric were $96.3 \%$ and $3.7 \%$, respectively. (e) Ribbon representation of the linker-less (LL) CaMKII crystal structure (PDBID 3SOA) ${ }^{23}$ (left), corresponding back-projection calculated at $20 \AA$ (middle), and representative class-average of the hub complex (right). The overall shape of the back-projected crystal structure looks similar to the $2 \mathrm{D}$ class average of the hub-complex, but with an increased radius of $\sim 100 \AA$ (compare middle and right). Focused $2 \mathrm{D}$ classification (mask $=110 \AA$ outer radius) does not identify any structures corresponding to the dimensions of the crystallized (LL) CaMKII holoenzyme in our dataset.
\end{abstract}


Focused 2D Classification of Extended Kinase Domains

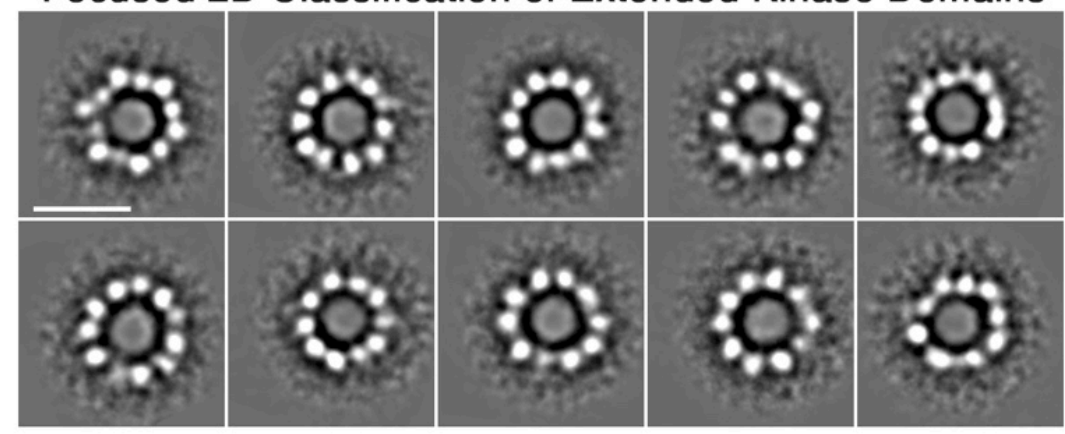

Supplemental Figure S4: Focused 2D classification of kinase domain arrangements. A set of representative $2 \mathrm{D}$ class averages obtained from negatively stained CaMKII particle images using an applied inner mask (inner diameter $=50 \AA$ ) to remove contributions from the central hub feature. Scale bar $=25 \mathrm{~nm}$. The resulting projection averages display clearly resolved kinase domain densities arranged along a radius of $\sim 24-28 \mathrm{~nm}$. These most populated classes reveal $9-12$ resolved densities. 
a
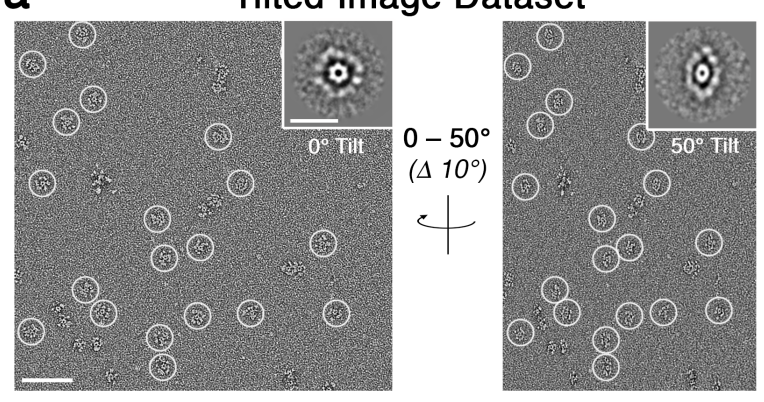

b Preliminary Reconstruction

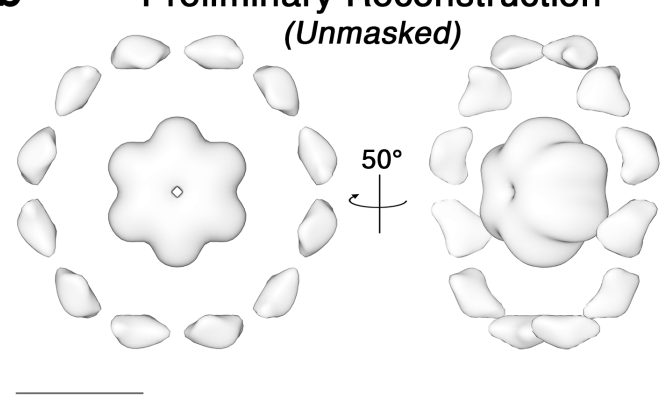

C

d 3D Mask

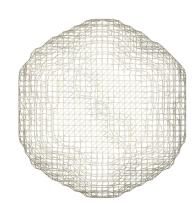

Masked Refinement (Hub Domain)

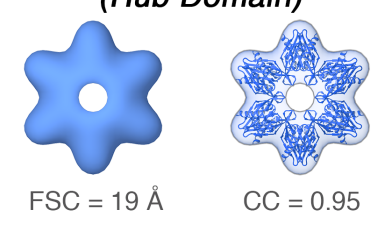

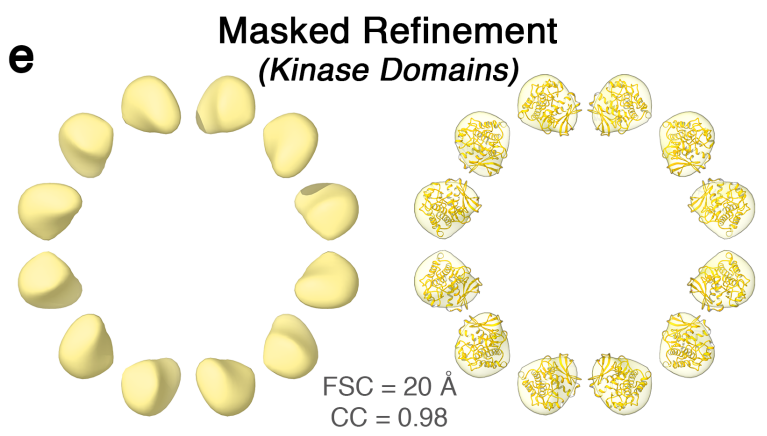

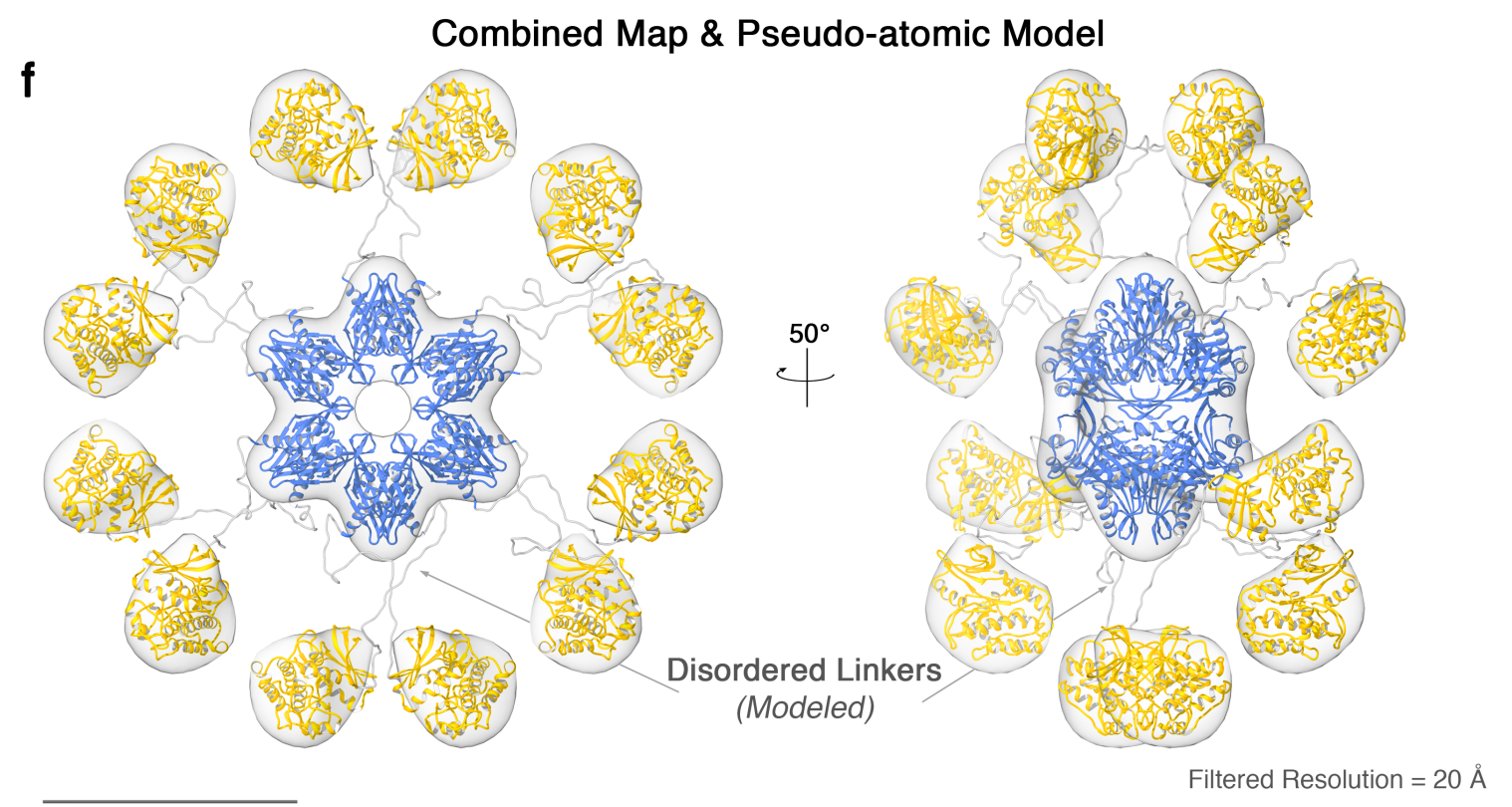


Supplementary Figure 5: Workflow of 3D reconstruction and pseudoatomic modeling of the CaMKIla holoenzyme. (a) Representative micrographs of negatively stained CaMKIla particles recorded with the specimen stage tilted at $0^{\circ}$ (left) and $50^{\circ}$ (right). Individual particles are indicated by white circles. A complete image dataset of $\sim 16,000$ particles were collected at varying stage tilts of $0^{\circ}, 10^{\circ}, 20^{\circ}, 30^{\circ}, 40^{\circ}$ and $50^{\circ}$. Scale bar $=$ $100 \mathrm{~nm}$. Insets, show representative reference-free 2D class averages. Scale bar $=25 \mathrm{~nm}$. (b) Initial $3 \mathrm{D}$ reconstruction obtained from unmasked particles using EMAN2 ${ }^{48}$. Scale bar $=100 \AA$. (c - e) Masked 3D refinement strategy. Scale bar $=100 \AA$. (c) $3 \mathrm{D}$ mask used for refinements of the central hub complex and peripheral kinase domains performed in RELION v1.4 ${ }^{49}$. (d) EM map of the hub domain complex obtained using the 3D mask in panel $\mathbf{c}$. The map was refined to $19 \AA$ (gold standard FSC) with applied 6 -fold dihedral symmetry as defined by the hub complex. The crystal structure of the human dodecameric CaMKIla hub complex (blue ribbon, residues 345 - 472; PDBID $5 I \mathrm{GS}^{22}$ ) fit well with the EM density map (correlation coefficient $=0.95$ at $20 \AA$ ). (e) EM map of the CaMKIla kinase domains obtained using an inverted 3D mask shown in panel c. The map was refined to $20 \AA$ (gold standard FSC) with applied 6-fold dihedral symmetry. The crystal structure of the isolated human CaMKIla kinase/regulatory domain (yellow ribbon, residues $13-300$; PDBID 2VZ6 ${ }^{21}$ ) fit well within each of the twelve EM densities (correlation coefficient $=0.98$ at $20 \AA$ ). (f) $A$ final EM map was constructed by merging EM densities shown in panels $\mathbf{d}$ and $\mathbf{e}$. A pseudo-atomic model of the CaMKIla holoenzyme was completed by connecting the crystal structures fit into the EM density map by random coil chains. Each kinase/regulatory domain (residues $13-300$ ) is connected to the nearest hub domain (residues $345-472$ ) using a disordered linker (grey coil, residues $301-344$ ). The disordered linker for each peptide chain was calculated separately using MODELER ${ }^{25}$. Scale bar = $100 \AA$. 

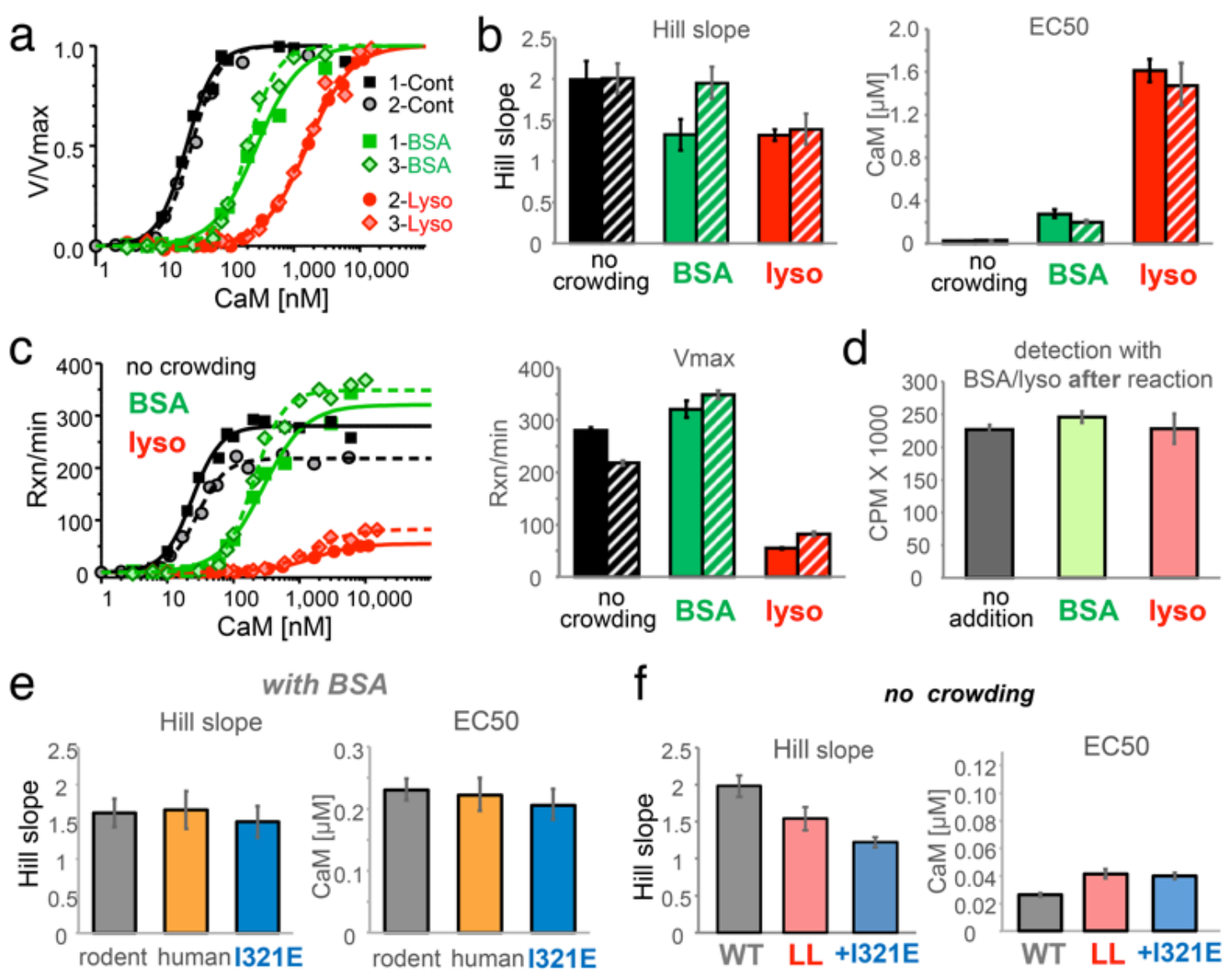

f

no crowding
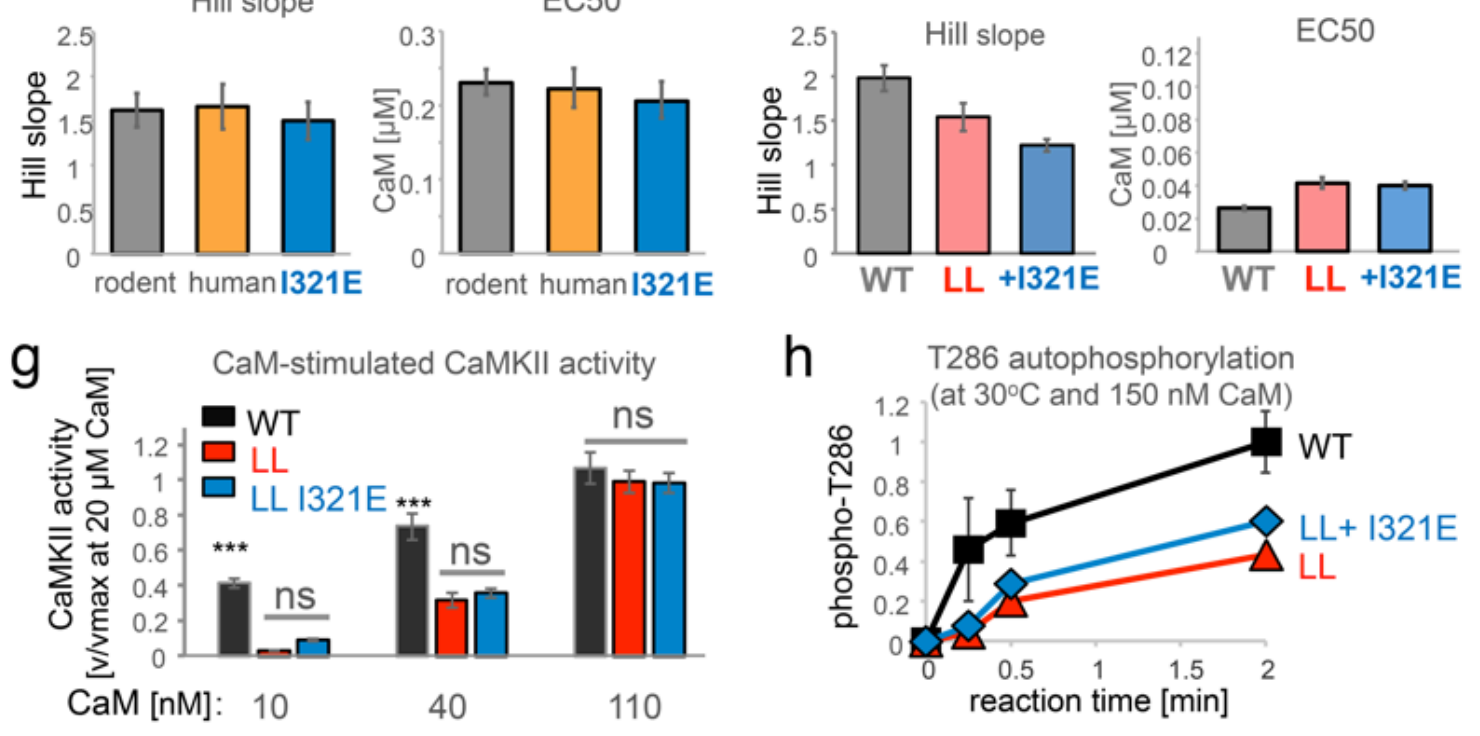

i T286 autophosphorylation ( $30 \mathrm{sec}$ at $30^{\circ} \mathrm{C}$ and $\left.150 \mathrm{nM} \mathrm{CaM}\right)$
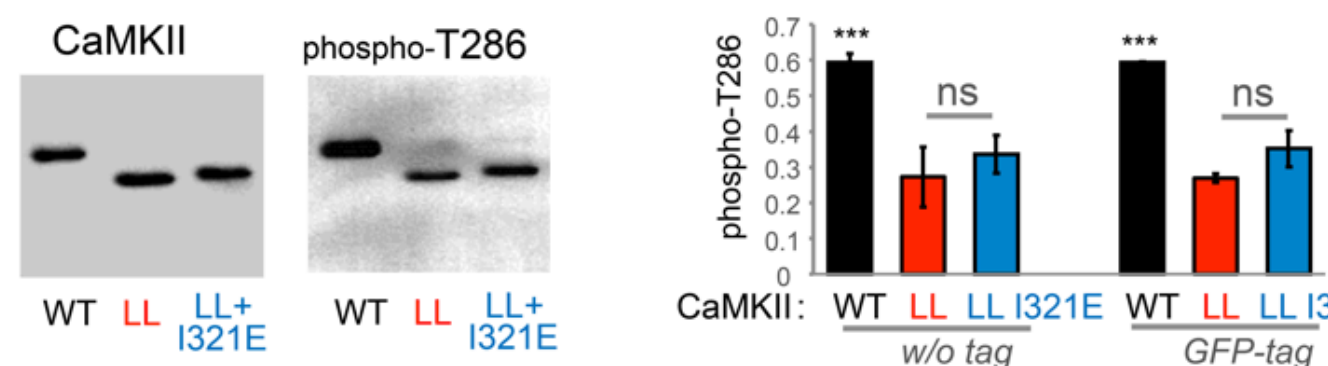

CaMKII: $\frac{\text { WT LL LL I321E }}{\text { w/o tag }} \frac{\text { WT LL LL I321E }}{\text { GFP-tag }}$ 
Supplementary Figure 6: CaMKIla activation by $\mathrm{Ca}^{2+} / \mathrm{CaM}$ in vitro and the effects of molecular crowding (by $150 \mathrm{mg} / \mathrm{ml}$ BSA or lysozyme), a linkerless deletion mutant (LL; described to favor the compact conformation), and an I321E mutant (described to prevent the compact conformation). (a, b) $\mathrm{Ca}^{2+} / \mathrm{CaM}$ dose/response curves for in vitro activation of rodent CaMKII without crowding (Contr) or with crowding by $150 \mathrm{mg} / \mathrm{ml} \mathrm{BSA}$ or lysozyme (lys) was determined in two independent experiments for each condition (with two conditions tested in parallel in each of three experiments, as indicated). While Fig. 5b,c show fits for the combined data points, here, curve fits and analysis are shown separately for each experiment. While the Hill slope is a sensitive measure, any indication for variability was seen only for BSA. Importantly, no increase in the Hill slope by molecular crowding was detected in any of the individual experiments. (c) Dose response as in panel a, but plotted as absolute kinase activity (in reactions per minute) instead of activity normalized to $V_{\max }$. This indicated that $V_{\max }$ was slightly increased by crowding with BSA, but strongly decreased by lysozyme. (d) Addition of BSA or lysozyme after the kinase reaction (but before detection of the phosphorylated substrate) did not affect detection of the phosphorylated substrate. Thus, lysozyme affected the $V_{\max }$ in the kinase reaction, not the detection after the reaction. (e) Rodent, human, and human I321E mutant CaMKIla showed identical Hill slope and $\mathrm{EC}_{50}$ during molecular crowding with BSA. Shown is the analysis of the dose response curves in Fig. 5d. (f) Without crowding, the linker-less mutant (LL) did not show any increase in Hill slope, thus indicating no compact conformation, at least when expressed in mammalian cells. The $\mathrm{EC}_{50}$ of the linker-less mutant showed only a very mild $\sim 1.5$ fold increase, and this was identical for the linker-less I321E mutant. Thus, the linker-less mutant was not in a compact conformation without molecular crowding; however, with molecular crowding, a compact conformation could be induced (see Fig. 5e, f). (g) An independent experiment verified identical $\mathrm{Ca}^{2+} / \mathrm{CaM}$ dose response for the linker-less mutant and its I321E mutant without molecular crowding. Compared to full-length CaMKIla wild type, a reduced response was seen at lower CaM concentrations (10 and $40 \mathrm{nM}$ ), but not at higher CaM concentration (100 nM), consistent with a rather mild shift in $\mathrm{Ca}^{2+} / \mathrm{CaM}$ sensitivity. Kinase activity was normalized to maximal activity induced with 20 $\mu \mathrm{M}$ CaM. (h) Time course of CaMKII T286 autophosphorylation at $30^{\circ} \mathrm{C}$ with 1 $\mathrm{mM} \mathrm{Ca}^{2+}$ and $150 \mathrm{nM} \mathrm{CaM}$. Autophosphorylation was detected by Western blot analysis and quantified as described (see also panel i). (i) Compared to CaMKIla wild type, T286 auto-phosphorylation was reduced to the same extent for linker-less CaMKII and its I321E mutant. The same result was obtained for GFP-tagged versions of the kinase. The autophosphorylation conditions chosen ( $30 \mathrm{sec}$ at $30^{\circ} \mathrm{C}$ with $150 \mathrm{nM}$ CaM) induced sub-maximal phosphorylation for CaMKII wild type (here $\sim 60 \%$ compared to extended 2 min reactions; see panel (cont'd page 170) 
Supplementary Figure 6, cont'd. h). Autophosphorylation was detected by Western blot analysis (left) and quantified (right) as described. Error bars in panels b-f represent the calculated standard error from the curve fits; error bars in panels g-i represent the standard error of mean (s.e.m.).

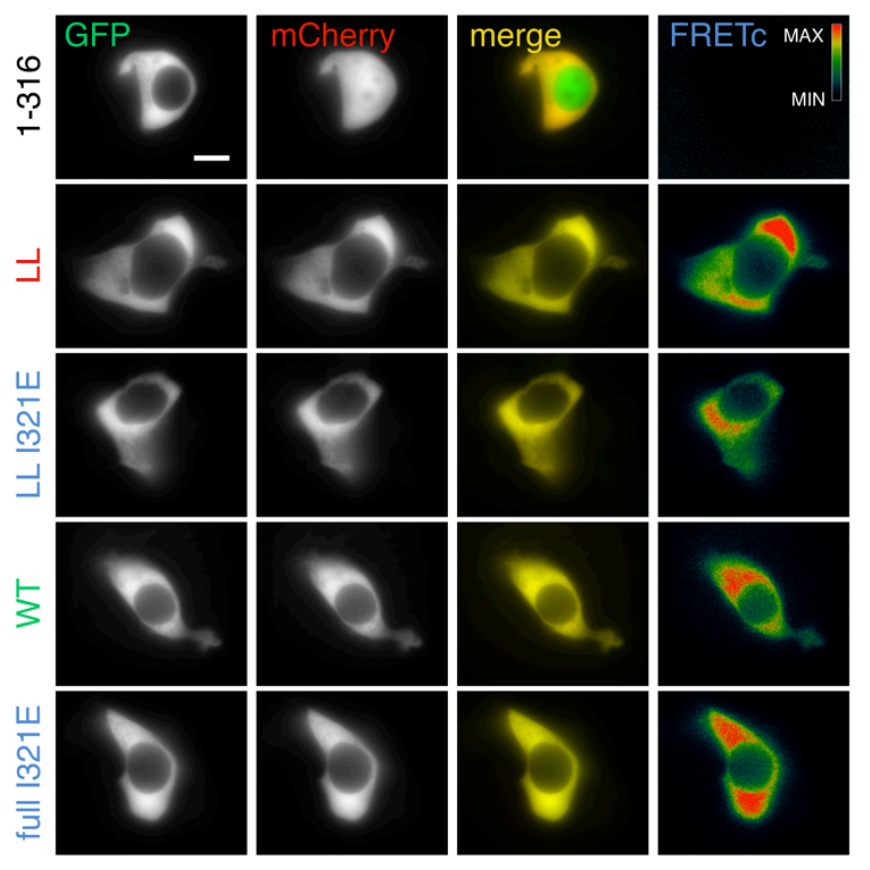

\section{Supplementary Figure 7: A FRET-based assay indicated a compact conformation within cells only for linker-less but not for full-length CaMKIla wild type. Shown here are example images for all conditions quantified in Fig. 6 of main text. This includes a truncated CaMKII control without the association domain (1-316), the linker-less kinase (LL), the linker- less kinase with the I321E mutation described to disrupt the compact conformation (LL 1321E), full-length wild type kinase (WT), and full-length kinase with the I321E mutation (full I321E). Scale bar $=10 \mathrm{~mm}$.}




\begin{tabular}{|l|l|c|l|c|c|}
\hline \multirow{2}{*}{ CaMKII } & \multirow{2}{*}{ exp. } & \multicolumn{2}{|c|}{$\boldsymbol{E C}_{50}[\mathrm{nM}]$} & \multicolumn{2}{c|}{ Hill Coefficient } \\
& & Without BSA & With BSA & Without BSA & With BSA \\
\hline rodent & 1 & $23.8+1.58 /-1.48$ & $276.7+43.0 /-37.21$ & $2.00+/-0.227$ & $1.32+/-0.192$ \\
\hline & 2 & $28.4+1.66 /-1.57$ & $203.2+13.0 /-12.21$ & $2.08+/-0.182$ & $1.95+/-0.192$ \\
\hline & 3 & nd & $231.2+17.7 /-16.42$ & nd & $1.62+/-0.189$ \\
\hline human & & nd & $222.3+27.8 /-24.68$ & nd & $1.66+/-0.253$ \\
\hline $\begin{array}{l}\text { human } \\
\text { I321E }\end{array}$ & & nd & $206.0+26.2 /-23.22$ & nd & $1.50+/-0.210$ \\
\hline
\end{tabular}

Supplementary Table 1. Molecular crowding with $150 \mathrm{mg} / \mathrm{ml}$ BSA does not increase the Hill coefficient for mammalian-expressed CaMKIla. If any, a slight decrease is observed. The significant increase in $\mathrm{EC}_{50}$ caused by molecular crowding is not related to induction of the compact conformation (at least for the full-length kinase tested in this Table), as it is indistinguishable for the I321E mutant that is incompetent for the compact conformation. Effect on rodent versus human CaMKII was also indistinguishable.

\begin{tabular}{|l|l|c|l|c|l|}
\hline \multirow{2}{*}{ CaMKII $\alpha$} & \multirow{2}{*}{ exp. } & \multicolumn{2}{|c|}{$\boldsymbol{E C}_{\mathbf{5 0}}$ [nM] } & \multicolumn{2}{c|}{ Hill Coefficient } \\
& & Without BSA & With BSA & Without BSA & With BSA \\
\hline LL & 1 & $42+3.4 /-3.2$ & $2570+668 /-530$ & $1.54+/-0.156$ & $0.99+/-0.091$ \\
\hline & 2 & nd & $1841+655 /-483$ & nd & $1.10+/-0.185$ \\
\hline LL + I321E & 1 & $40+2.3 /-2.2$ & $849+159 /-133$ & $1.22+/-0.070$ & $1.06+/-0.119$ \\
\hline & 2 & nd & $1099+106 /-96$ & nd & $1.18+/-0.083$ \\
\hline
\end{tabular}

Supplementary Table 2. Molecular crowding with $150 \mathrm{mg} / \mathrm{ml}$ BSA might induce a compact conformation for linker-less (LL) CaMKII. However, the differential effect of crowding on the I321E mutant that is incompetent for the compact conformation manifested in $\mathrm{EC}_{50}$ rather than the Hill coefficient.

\section{Supplementary Movie Legend}

Supplementary Movie 1: 3D reconstruction and pseudo-atomic modeling of the CaMKIla holoenzyme 


\section{References}

1. Coultrap, S.J. \& Bayer, K.U. CaMKII regulation in information processing and storage. Trends Neurosci 35, 607-18 (2012).

2. Lisman, J., Yasuda, R. \& Raghavachari, S. Mechanisms of CaMKII action in long-term potentiation. Nat Rev Neurosci 13, 169-82 (2012).

3. Hell, J.W. CaMKII: claiming center stage in postsynaptic function and organization. Neuron 81, 249-65 (2014).

4. Giese, K.P., Fedorov, N.B., Filipkowski, R.K. \& Silva, A.J. Autophosphorylation at Thr286 of the alpha calcium-calmodulin kinase II in LTP and learning. Science 279, 870-3 (1998).

5. Coultrap, S.J. et al. Autonomous CaMKII mediates both LTP and LTD using a mechanism for differential substrate site selection. Cell Rep $\mathbf{6}$, 431-7 (2014).

6. Bayer, K.U., De Koninck, P., Leonard, A.S., Hell, J.W. \& Schulman, H. Interaction with the NMDA receptor locks CaMKII in an active conformation. Nature 411, 801-5 (2001).

7. Barcomb, K., Hell, J.W., Benke, T.A. \& Bayer, K.U. The CaMKII/GluN2B Protein Interaction Maintains Synaptic Strength. J Biol Chem 291, 16082-9 (2016).

8. Halt, A.R. et al. CaMKII binding to GluN2B is critical during memory consolidation. EMBO J 31, 1203-16 (2012). 
9. Strack, S., McNeill, R.B. \& Colbran, R.J. Mechanism and regulation of calcium/calmodulin-dependent protein kinase II targeting to the NR2B subunit of the N-methyl-D-aspartate receptor. J Biol Chem 275, 23798806 (2000).

10. Bayer, K.U. et al. Transition from reversible to persistent binding of CaMKII to postsynaptic sites and NR2B. J Neurosci 26, 1164-74 (2006).

11. Hanson, P.I., Meyer, T., Stryer, L. \& Schulman, H. Dual role of calmodulin in autophosphorylation of multifunctional CaM kinase may underlie decoding of calcium signals. Neuron 12, 943-56 (1994).

12. Rich, R.C. \& Schulman, H. Substrate-directed function of calmodulin in autophosphorylation of Ca2+/calmodulin-dependent protein kinase II. $J$ Biol Chem 273, 28424-9 (1998).

13. De Koninck, P. \& Schulman, H. Sensitivity of CaM kinase II to the frequency of Ca2+ oscillations. Science 279, 227-30 (1998).

14. Kim, K. et al. A Temporary Gating of Actin Remodeling during Synaptic Plasticity Consists of the Interplay between the Kinase and Structural Functions of CaMKII. Neuron 87, 813-26 (2015).

15. Borgesius, N.Z. et al. betaCaMKII plays a nonenzymatic role in hippocampal synaptic plasticity and learning by targeting alphaCaMKII to synapses. J Neurosci 31, 10141-8 (2011). 
16. O'Leary, H., Lasda, E. \& Bayer, K.U. CaMKIlbeta association with the actin cytoskeleton is regulated by alternative splicing. Mol Biol Cell 17, 4656-65 (2006).

17. Meador, W.E., Means, A.R. \& Quiocho, F.A. Modulation of calmodulin plasticity in molecular recognition on the basis of $\mathrm{x}$-ray structures. Science 262, 1718-21 (1993).

18. Hoelz, A., Nairn, A.C. \& Kuriyan, J. Crystal structure of a tetradecameric assembly of the association domain of $\mathrm{Ca} 2+/$ calmodulin-dependent kinase II. Mol Cell 11, 1241-51 (2003).

19. Rosenberg, O.S., Deindl, S., Sung, R.J., Nairn, A.C. \& Kuriyan, J. Structure of the autoinhibited kinase domain of CaMKII and SAXS analysis of the holoenzyme. Cell 123, 849-60 (2005).

20. Chao, L.H. et al. Intersubunit capture of regulatory segments is a component of cooperative CaMKII activation. Nat Struct Mol Biol 17, 264$72(2010)$.

21. Rellos, P. et al. Structure of the CaMKIldelta/calmodulin complex reveals the molecular mechanism of CaMKII kinase activation. PLoS Biol 8, e1000426 (2010).

22. Bhattacharyya, M. et al. Molecular mechanism of activation-triggered subunit exchange in $\mathrm{Ca}(2+) /$ calmodulin-dependent protein kinase II. Elife 5(2016). 
23. Chao, L.H. et al. A mechanism for tunable autoinhibition in the structure of a human Ca2+/calmodulin- dependent kinase II holoenzyme. Cell 146, 732-45 (2011).

24. Kanaseki, T., Ikeuchi, Y., Sugiura, H. \& Yamauchi, T. Structural features of $\mathrm{Ca} 2+/$ calmodulin-dependent protein kinase II revealed by electron microscopy. J Cell Biol 115, 1049-60 (1991).

25. Fiser, A., Do, R.K. \& Sali, A. Modeling of loops in protein structures. Protein Sci 9, 1753-73 (2000).

26. Hoffman, L., Stein, R.A., Colbran, R.J. \& McHaourab, H.S. Conformational changes underlying calcium/calmodulin-dependent protein kinase II activation. EMBO J 30, 1251-62 (2011).

27. Drozdetskiy, A., Cole, C., Procter, J. \& Barton, G.J. JPred4: a protein secondary structure prediction server. Nucleic Acids Res 43, W389-94 (2015).

28. Flory, P.J. Spatial configuration of macromolecular chains. Science 188, 1268-76 (1975).

29. Thaler, C., Koushik, S.V., Puhl, H.L., 3rd, Blank, P.S. \& Vogel, S.S. Structural rearrangement of CaMKIlalpha catalytic domains encodes activation. Proc Natl Acad Sci U S A 106, 6369-74 (2009). 
30. Goodell, D.J., Eliseeva, T.A., Coultrap, S.J. \& Bayer, K.U. CaMKII binding to GluN2B is differentially affected by macromolecular crowding reagents. PLoS One 9, e96522 (2014).

31. Bayer, K.U., De Koninck, P. \& Schulman, H. Alternative splicing modulates the frequency-dependent response of $\mathrm{CaMKII}$ to $\mathrm{Ca}(2+)$ oscillations. EMBO J 21, 3590-7 (2002).

32. Gaertner, T.R. et al. Comparative analyses of the three-dimensional structures and enzymatic properties of alpha, beta, gamma and delta isoforms of Ca2+-calmodulin-dependent protein kinase II. J Biol Chem 279, 12484-94 (2004).

33. Kolodziej, S.J., Hudmon, A., Waxham, M.N. \& Stoops, J.K. Threedimensional reconstructions of calcium/calmodulin-dependent (CaM) kinase Ilalpha and truncated CaM kinase Ilalpha reveal a unique organization for its structural core and functional domains. J Biol Chem 275, $14354-9$ (2000).

34. Bradshaw, J.M., Kubota, Y., Meyer, T. \& Schulman, H. An ultrasensitive $\mathrm{Ca} 2+/$ calmodulin-dependent protein kinase II-protein phosphatase 1 switch facilitates specificity in postsynaptic calcium signaling. Proc Natl Acad Sci U S A 100, 10512-7 (2003).

35. Woodgett, J.R., Davison, M.T. \& Cohen, P. The calmodulin-dependent glycogen synthase kinase from rabbit skeletal muscle. Purification, subunit structure and substrate specificity. Eur J Biochem 136, 481-7 (1983). 
36. Morris, E.P. \& Torok, K. Oligomeric structure of alpha-calmodulindependent protein kinase II. J Mol Biol 308, 1-8 (2001).

37. Rosenberg, O.S. et al. Oligomerization states of the association domain and the holoenyzme of Ca2+/CaM kinase II. FEBS J 273, 682-94 (2006).

38. Stratton, M. et al. Activation-triggered subunit exchange between CaMKII holoenzymes facilitates the spread of kinase activity. Elife 3, e01610 (2014).

39. Takao, K. et al. Visualization of synaptic $\mathrm{Ca} 2+$ /calmodulin-dependent protein kinase II activity in living neurons. J Neurosci 25, 3107-12 (2005).

40. Shibata, A.C., Maebashi, H.K., Nakahata, Y., Nabekura, J. \& Murakoshi, H. Development of a molecularly evolved, highly sensitive CaMKII FRET sensor with improved expression pattern. PLoS One 10, e0121109 (2015).

41. Singla, S.I., Hudmon, A., Goldberg, J.M., Smith, J.L. \& Schulman, H. Molecular characterization of calmodulin trapping by calcium/calmodulindependent protein kinase II. J Biol Chem 276, 29353-60 (2001).

42. Coultrap, S.J., Buard, I., Kulbe, J.R., Dell'Acqua, M.L. \& Bayer, K.U. CaMKII autonomy is substrate-dependent and further stimulated by Ca2+/calmodulin. J Biol Chem 285, 17930-7 (2010).

43. Coultrap, S.J. \& Bayer, K.U. Ca2+/Calmodulin-Dependent Protein Kinase II (CaMKII). in Neuromethods: Protein Kinase Technologies (ed. Mukai, H.) 49-72 (Springer, 2012). 
44. Coultrap, S.J., Barcomb, K. \& Bayer, K.U. A significant but rather mild contribution of T286 autophosphorylation to Ca2+/CaM-stimulated CaMKII activity. PLoS One 7, e37176 (2012).

45. Coultrap, S.J. \& Bayer, K.U. Nitric oxide induces Ca2+-independent activity of the Ca2+/calmodulin-dependent protein kinase II (CaMKII). $J$ Biol Chem 289, 19458-65 (2014).

46. Sorkin, A., McClure, M., Huang, F. \& Carter, R. Interaction of EGF receptor and grb2 in living cells visualized by fluorescence resonance energy transfer (FRET) microscopy. Curr Biol 10, 1395-8 (2000).

47. Smith, F.D. et al. Intrinsic disorder within an AKAP-protein kinase A complex guides local substrate phosphorylation. Elife 2, e01319 (2013).

48. Tang, G. et al. EMAN2: an extensible image processing suite for electron microscopy. J Struct Biol 157, 38-46 (2007).

49. Scheres, S.H. RELION: implementation of a Bayesian approach to cryoEM structure determination. J Struct Biol 180, 519-30 (2012).

50. Pettersen, E.F. et al. UCSF Chimera--a visualization system for exploratory research and analysis. Journal of computational chemistry $\mathbf{2 5}$, 1605-12 (2004). 


\section{Chapter 5}

\section{Multivalency regulates activity in an intrinsically disordered transcription}

factor

This chapter has been published

Multivalency regulates activity in an intrinsically disordered transcription factor. eLife (2018).Sarah A. Clark ${ }^{1}$, Janette B. Myers ${ }^{2}$, Ashleigh King ${ }^{3}$, Radovan Fiala $^{4}$, Jiri Novacek ${ }^{4}$, F. Grant Pearce ${ }^{5}$, Jörg Heierhorst ${ }^{3}$, Steve L. Reichow ${ }^{2}$, and Elisar Barbar ${ }^{1}$.

${ }^{1}$ Department of Biochemistry and Biophysics, Oregon State University, Corvallis, Oregon 97331

${ }^{2}$ Department of Chemistry, Portland State University, Portland, Oregon 97201

${ }^{3}$ St. Vincent's Institute of Medical Research, and Department of Medicine St. Vincent's Health, The University of Melbourne, Fitzroy, Victoria 3065, Australia ${ }^{4}$ Central European Institute of Technology, Masaryk University, Kamenice 753/5, 62500 Brno, Czech Republic

${ }^{5}$ School of Biological Sciences, University of Canterbury, Christchurch, New Zealand 4800

This work was a collaboration between the Reichow lab and Dr. Elisar Barbar's lab. Protein samples were provided by Dr. Barbar's lab. Janette Myers prepared electron microscopy grids, screened conditions and collected data, as well as performed image analysis. Sarah Clark and other authors performed all other experiments. 


\section{Abstract}

The transcription factor ASCIZ (ATMIN, ZNF822) has an unusually high number of recognition motifs for the product of its main target gene, the hub protein LC8 (DYNLL1). Using a combination of biophysical methods, structural analysis by NMR and electron microscopy, and cellular transcription assays, we developed a model that proposes a concerted role of intrinsic disorder and multiple LC8 binding events in regulating LC8 transcription. We demonstrate that the long intrinsically disordered C-terminal domain of ASCIZ binds LC8 to form a dynamic ensemble of complexes with a gradient of transcriptional activity that is inversely proportional to LC8 occupancy. The preference for low occupancy complexes at saturating LC8 concentrations with both human and Drosophila ASCIZ indicates that negative cooperativity is an important feature of ASCIZ-LC8 interactions. The prevalence of intrinsic disorder and multivalency among transcription factors suggests that formation of heterogeneous, dynamic complexes is a widespread mechanism for tuning transcriptional regulation.

\section{Impact Statement}

Binding of multiple LC8 copies to the intrinsically disordered region of the transcription factor ASCIZ exemplifies a new and potentially widespread molecular mechanism for negative feedback regulation. 


\section{Introduction}

Regulation of transcriptional activity is essential for every biological process. Common mechanisms for regulation include post-translational modifications and/or cooperativity among multiple activators and repressors (Banerjee, 2003). Some transcription factors contain multiple regulatory sites for either posttranslational modifications (Meek \& Anderson, 2009) or binding partners (Cantor \& Orkin, 2002), and their activity is thus tuned by the combined action of these components. Recent studies have revealed a high degree of intrinsic disorder in transcription factors, indicating that the inherent dynamical behavior harbored by these structures is critical for these regulatory events to take place (J. Li et al., 2017; Liu et al., 2006; Minezaki, Homma, Kinjo, \& Nishikawa, 2006). Our developing understanding suggests that the intrinsically disordered domains in transcription factors may provide a multivalent platform for the recruitment of regulatory binding partners (Currie et al., 2017; Shammas, 2017). While the functional consequence of multivalent binding to an intrinsically disordered region has been described for a few transcription systems (Dyson \& Wright, 2016; Uversky et al., 2009), it remains unclear for the vast majority of cases.

Human ASCIZ (ATMIN-Substrate Chk-Interacting $\mathrm{Zn}^{2+}$ finger) is an $88 \mathrm{kDa}$ protein that has recently been identified as a transcription factor for the hub protein, LC8 (dynein light chain 8)(Jurado, Conlan, et al., 2012). Mice with mutations in $\mathrm{ASCIZ}$ that prevent LC8 transcription die in late embryogenesis and exhibit serious developmental defects in kidneys and lungs (Goggolidou, Hadjirin, 
et al., 2014; Goggolidou, Stevens, et al., 2014; Jurado et al., 2010). Drosophila ASCIZ knockouts die in early embryogenesis and localized knockdowns using RNAi show mitotic defects (Zaytseva et al., 2014). Mutant phenotypes in Drosophila, developing mouse B lymphocytes, and cultured cells are rescued by ectopic overexpression of LC8, demonstrating that the observed defects of ASCIZ knockouts are due to ASCIZ regulation of LC8 expression (Goggolidou, Stevens, et al., 2014; Jurado, Gleeson, et al., 2012; Zaytseva et al., 2014). In addition, it has recently been shown that a conditional knockout of LC8 almost perfectly copies the corresponding phenotypes of $\mathrm{ASCIZ}$ knockouts in mouse B cell development and B cell lymphomagenesis (King et al., 2017; Wong et al., 2016).

LC8 is a highly conserved $20.6 \mathrm{kDa}$ protein homodimer (10.3 kDa monomer) that facilitates self-association of its primarily disordered partners (Barbar, 2008; Barbar \& Nyarko, 2014; S. A. Clark, Jespersen, Woodward, \& Barbar, 2015) (Figure 1a). LC8 binding is associated with a range of cellular processes, from cell division to apoptosis, underscoring LC8's essential role as a regulatory hub (Dunsch et al., 2012; Puthalakath, Huang, O'Reilly, King, \& Strasser, 1999). LC8 preferentially binds to a 10 -amino acid motif in intrinsically disordered regions (IDRs) containing highly conserved TQT residues at positions 7-9 (Barbar, 2008; Rapali, Radnai, et al., 2011) (Figure 1b). In complex with LC8, the otherwise intrinsically disordered motif adopts a ß-strand conformation (Benison, Karplus, \& Barbar, 2007; Liang, Jaffrey, Guo, Snyder, \& Clardy, 1999) (Figure 1a,b). 
(a)

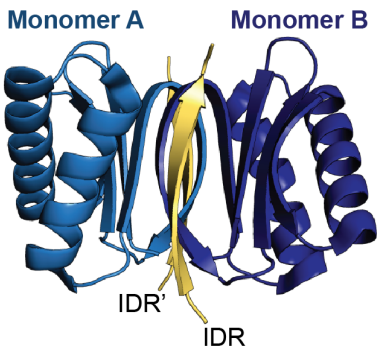

$\left.90^{\circ}-\right)$

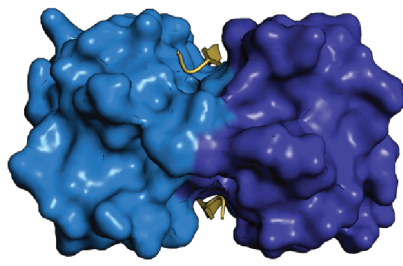

(b)

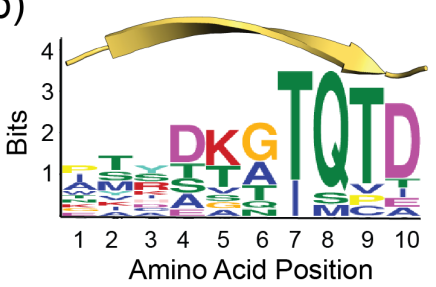

(c)

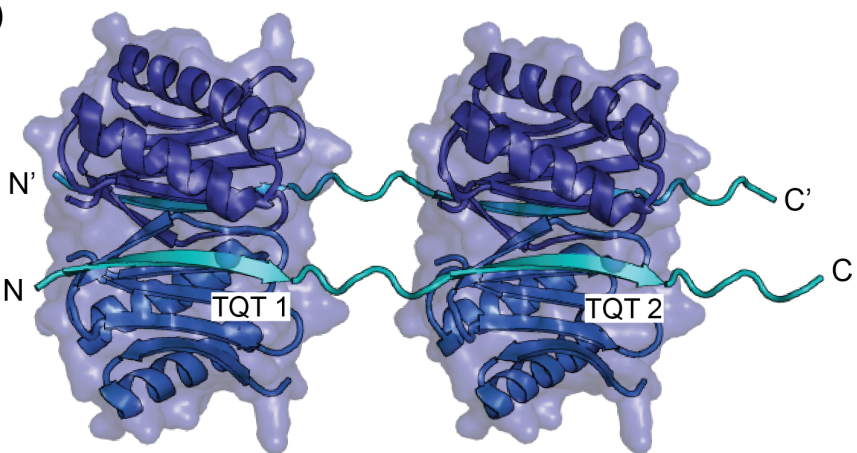

(d)
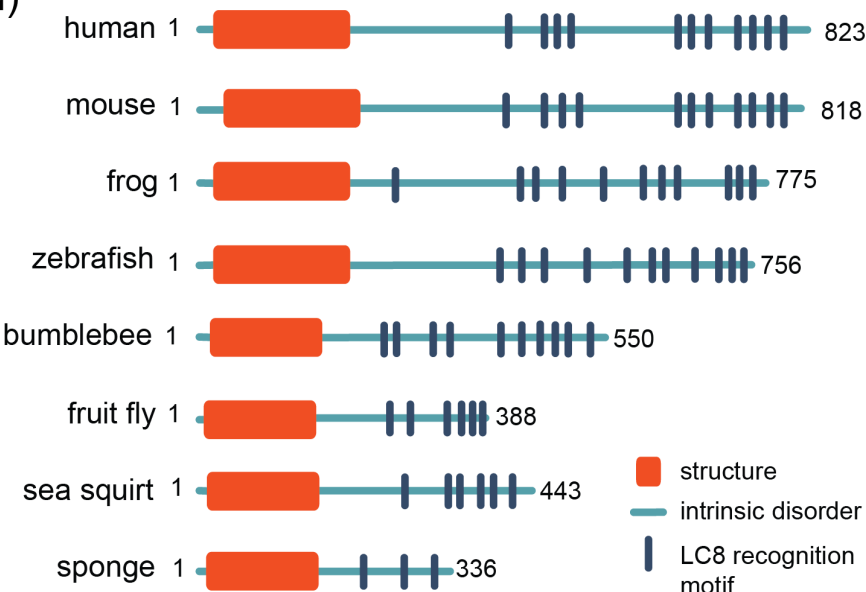

Figure 1. LC8 dimerizes its protein partners. (a) Ribbon diagram of dimeric LC8, where each monomer is colored a different shade of blue and bound to a representative peptide in yellow (PDB 5EOL). A single LC8 dimer binds two peptides (intrinsically disordered region, IDR), one on each side of the dimerization interface, that are arranged in a parallel fashion. (b) A sequence logo of LC8 binding motifs derived from sequences of the motifs in the 11 crystal structures reported for LC8/peptide complexes. Height of amino acids indicates their relative frequency at that position. (c) A crystal structure of two LC8 dimers bound to two copies of the same intrinsically disordered chains illustrates how LC8 can bind to multivalent partner proteins with two LC8 recognition motifs, TQT1 and TQT2 (PDB 3GLW). (d) Sequence-based predictions of order (red boxes), disorder (blue lines), and LC8 binding motifs (dark blue bars) are shown for $10 \mathrm{ASCIZ}$ proteins from the animal kingdom. ASCIZ proteins for different species were identified from a BLAST search (Boratyn et al., 2013) against the human protein. Sequence predictions of order and disorder were obtained with PSIPRED (McGuffin, Bryson, \& Jones, 2000), where a criteria for order based on $>10 \%$ probability of predicted structure in a 50+ amino acid stretch. LC8 binding sites for human ASCIZ and Drosophila ASCIZ were obtained from the literature (Rapali, Garcia-Mayoral, et al., 2011; Zaytseva et al., 2014). Putative LC8 binding sites for the other species were identified based on the presence of TQT residues. 
Analysis of the 11 crystal structures of LC8 bound to short peptides containing the motif explains why the TQT residues are essential for binding: the $Q$ is involved in interactions with both LC8 subunits in the dimer, while both T's are fully buried and thus evolutionarily constrained. In these interactions, TQT acts as the motif anchor while the other seven highly variable motif residues modulate affinity, as described in the anchored flexibility model of LC8 motif recognition (S. Clark, Nyarko, Lohr, Karplus, \& Barbar, 2016). LC8 binds one motif in each of its two symmetrical binding grooves (Figure 1a), creating an IDP duplex that serves as a bivalent scaffold. This scaffold aids in higher order complex assembly by promoting binding of other proteins, including additional LC8 dimers (Figure 1c), and enhancing self-association and oligomerization processes that often involve coiled-coil formation (Hall, Karplus, \& Barbar, 2009; Kidane et al., 2013). In recent years, the number of experimentally characterized LC8 partners has risen to more than 40, and prediction methods indicate that dozens more may specifically bind LC8 (Rapali, Radnai, et al., 2011). Gaining insight into how LC8 interacts with partner proteins, and how LC8 levels in the cell are balanced, is therefore paramount to understanding the regulation of many cellular processes.

A distinctive feature of $A S C I Z$ is the high number of LC8 recognition motifs within its C-terminal domain. Although some LC8 partners have multiple recognition motifs (Dunsch et al., 2012; Fejtova et al., 2009; Gupta, Diener, Sivadas, Rosenbaum, \& Yang, 2012; Stelter et al., 2007) (Nucleoporin Nup159 has 5, Chica and Bassoon each has 3), human ASCIZ contains 11 functional LC8 
binding sites (Rapali, Garcia-Mayoral, et al., 2011), the most by far of any partner protein identified to date. This enrichment in LC8-binding sites is conserved throughout the animal kingdom, underscoring the importance of multiple motifs in ASCIZ function (Figure 1d). Cell culture transcription assays demonstrate that ASCIZ regulates LC8 transcription via a system of negative autoregulation, for which the mechanism is not well understood. Disruption of the ASCIZ/LC8 interaction via mutation of the TQT sites results in an increased level of LC8 transcription, while overexpression of LC8 decreases transcription (Jurado, Conlan, et al., 2012). This observation led to the hypothesis that ASCIZ acts as a sensor for cellular LC8 and regulates LC8 transcription levels according to cellular needs (Jurado, Conlan, et al., 2012). As LC8 expression levels vary among tissue types (Chintapalli, Wang, \& Dow, 2007) and LC8 overexpression enhances the survival and proliferation of breast cancer cells in culture (Vadlamudi et al., 2004), regulation of LC8 levels is critical for cellular health and homeostasis. However, while high levels of LC8 inhibit ASCIZ transcriptional activity (Jurado, Conlan, et al., 2012), it is not known how this activity is controlled at the molecular level nor the requirement for multiple binding sites.

In this work, we use a combination of biophysical, structural, and molecular biology tools to explore the relationship between ASCIZ multivalency and LC8 transcription. We show that human and Drosophila ASCIZ bind to multiple LC8 dimers simultaneously to form a dynamic, heterogeneous mixture of complexes, of which low occupancy intermediates are highly populated. Transcriptional 
assays with human ASCIZ demonstrate that the number of bound LC8 dimers modulates ASCIZ transcription, creating a gradient of activity. These observations support a novel model of autoregulation, whereby $\mathrm{ASClZ}$ engages in a dynamic equilibrium of multivalent interactions that tune the level of $\mathrm{ASClZ}$ transcriptional activity.

\section{Results}

\section{Unbound ASCIZ Is a Primarily Disordered Monomer}

The $45 \mathrm{kDa}$ Drosophila ASCIZ protein, dASCIZ, is predicted to contain four Znfinger motifs at the $\mathrm{N}$-terminus followed by a 243-amino acid long region of intrinsic disorder. The disordered region has six predicted LC8 recognition sites identified by a canonical TQT motif (Figure 2a, dark blue bars): QT1 (residues 251-262), QT2 (274-285), QT4 (323-334), QT5 (340-351), QT6 (354-365), and QT7 (374-385). QT3 (285-296) lacks the TQT residues but is identified experimentally as an LC8 recognition site in this work (below). Purification of fulllength dASCIZ is impeded by poor expression levels and insolubility, and therefore we designed and produced constructs corresponding to the zinc finger domain, dZnF (residues 1-156, red bar Figure 2a), and the LC8-binding domain, dLBD (residues 241-388, Figure 2a).

Sedimentation velocity analysis of the $\mathrm{dZnF}$ and dLBD (Figure $2 \mathrm{~b}$ ) indicates that each is a monomer in solution with molecular weights of $17.4 \mathrm{kDa}$ and $18 \mathrm{kDa}$, respectively (theoretical MW $17.6 \mathrm{kDa}$ and $17 \mathrm{kDa}$ ). The CD spectrum of $\mathrm{dZnF}$ 
(a)

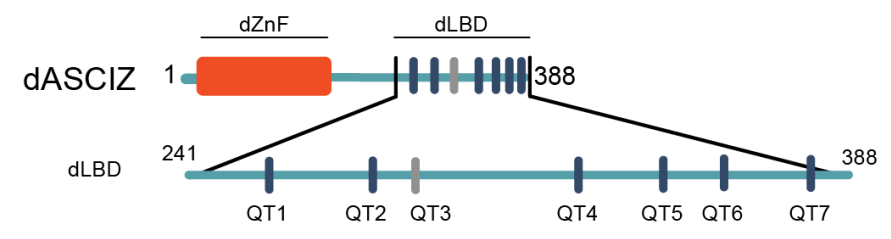

(b)

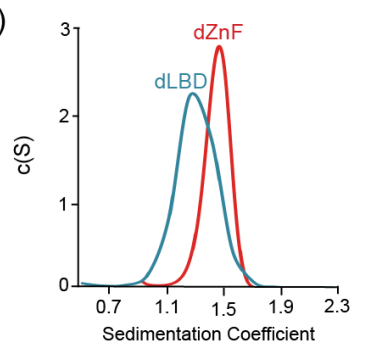

(d)

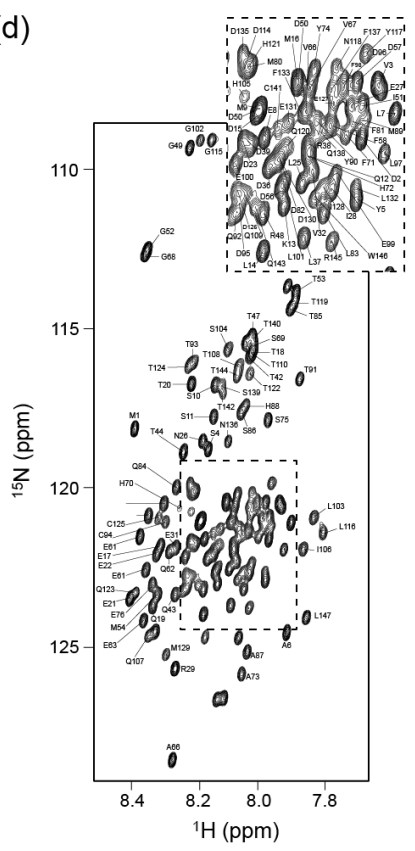

(c)

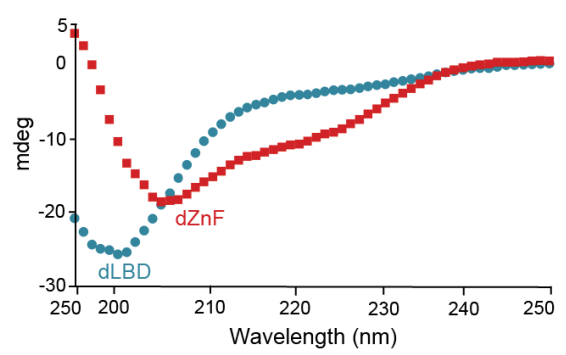

(e)

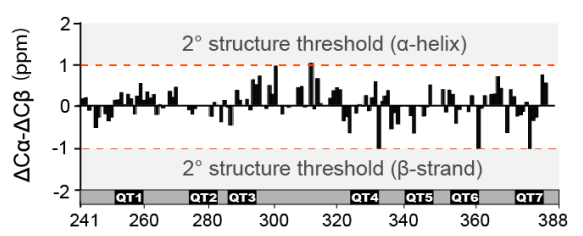

(f)

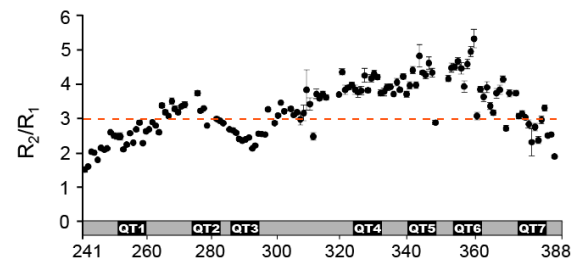

(g)

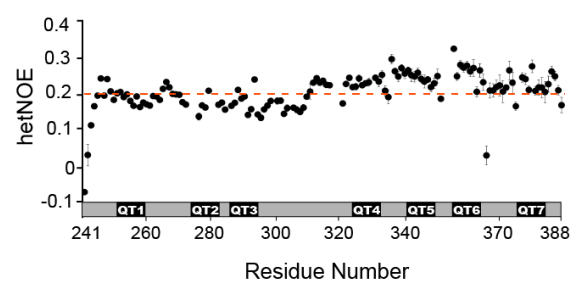

Figure 2. Structures of $A A S C I Z D N A$ and light chain binding domains. (a) Domain structure of $d A S C I Z$, showing the ZnF domain (red) and 7 LC8 binding motifs in its C-terminal domain (blue). Dark blue bars indicate predicted TQT motifs and gray bars indicate the TMT motif (QT3) identified in this study. (b) Sedimentation velocity analysis of the $\mathrm{ZnF}$ domain (red), collected at $10{ }^{\circ} \mathrm{C}$, and the $d L B D$ (blue), collected at $25^{\circ} \mathrm{C}$. (c) Far UV CD spectrum of the ZnF domain (red squares) and the $d L B D$ (blue circles), both collected at $10{ }^{\circ} \mathrm{C}$. (d) $\left[{ }^{15} \mathrm{~N}-{ }^{1} \mathrm{H}\right]$-BEST-TROSY spectrum at $850 \mathrm{MHz}$ showing backbone assignments for 133 non-proline residues. Unassigned peaks correspond to the two additional $N$-terminal residues from the expression vector. The spectrum was recorded at $10^{\circ} \mathrm{C}$. (cont'd page 181) 
shows a large negative ellipticity at $208 \mathrm{~nm}$ and a small negative ellipticity at 222 $\mathrm{nm}$ (Figure 2c, red) indicative of a mix of alpha helices and loops, similar to CD spectra of other ZnF proteins (Ezomo, 2010). The dLBD CD spectrum has a large negative ellipticity at $200 \mathrm{~nm}$, indicating that it is primarily disordered (Figure 2c, blue). From 5D NMR experiments, backbone assignments for $90 \%$ of the 148 residues in dLBD were obtained (Figure 2d). A high level of disorder in dLBD is revealed by the limited amide proton chemical shift dispersion in ${ }^{15} \mathrm{~N}$ HSQC spectra (Figure 2d), and a lack of secondary structure preference is further supported by small $\Delta \mathrm{C} \alpha-\Delta \mathrm{C} \beta$ chemical shift differences from random coil values (Figure 2e). Together these data demonstrate that $\mathrm{dASCIZ}$ contains an $\mathrm{N}$ terminal structured domain as well as a long intrinsically disordered domain, and constructs of each domain are monomeric in solution.

Local dynamics of dLBD were assessed by NMR measurement of the ${ }^{15} \mathrm{~N}$ longitudinal $\left(R_{1}\right)$, transverse $\left(R_{2}\right)$ relaxation, and ${ }^{1} \mathrm{H}^{15} \mathrm{~N}$ heteronuclear NOEs. $R_{2} / R_{1}$ values range from 1.5 to 5.3 with an average of 3.3 (Figure $2 f$ ). Relatively higher $R_{2} / R_{1}$ values for residues $321-363$ suggest motional restriction in this

Figure 2 cont'd (e) A plot of secondary chemical shift differences versus residue number. $\triangle C \alpha$ and $\triangle C \beta$ values were calculated by subtracting the random coil chemical shift from the experimentally determined chemical shift value (Tamiola, Acar, \& Mulder, 2010). $\Delta C \alpha-\Delta C \beta$ values $> \pm 1.0 \mathrm{ppm}$ are considered to be significant. (f) Plots of $R_{2} / R_{1}$ and (g) heteronuclear NOE values measured at $10{ }^{\circ} \mathrm{C}$ per residue. A dotted line is placed at the average value of both plots to aid in visualization. Segments corresponding to LC8 recognition motifs, QT1, QT2, QT3, QT4, QT5, QT6, and QT7 are shown. Additional structural characterization of the $d L B D$ by circular dichroism and gel filtration chromatography is shown in figure 2 - figure supplement 1. 
(a)
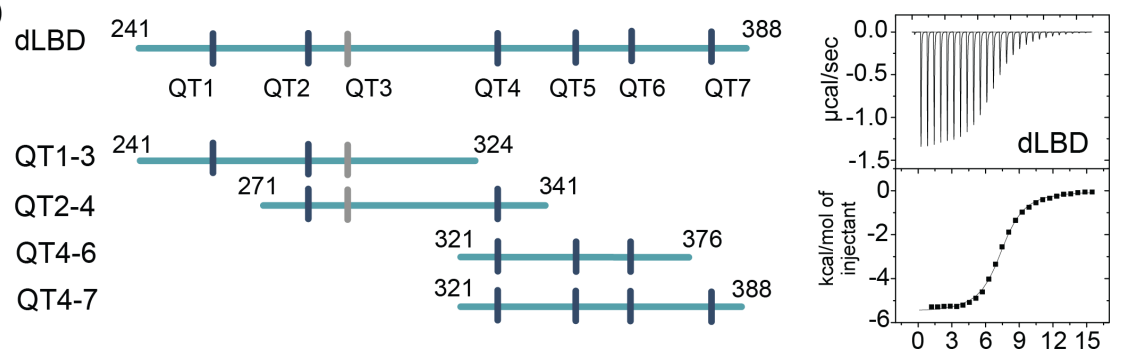

(b)
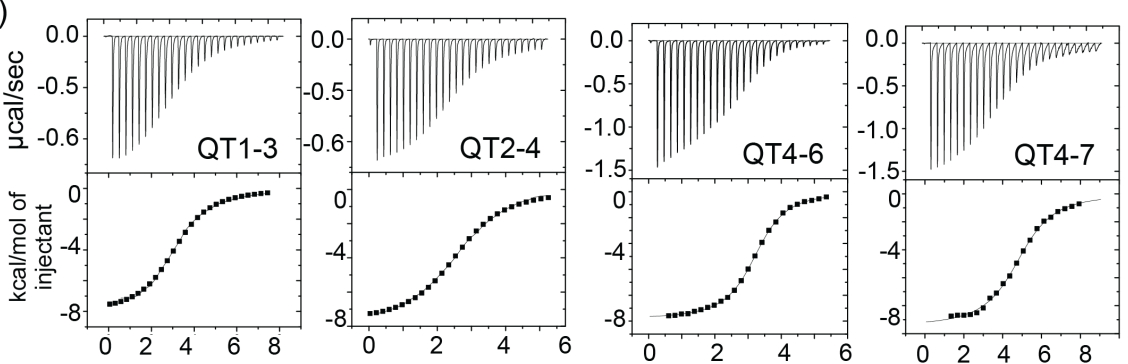

(c)
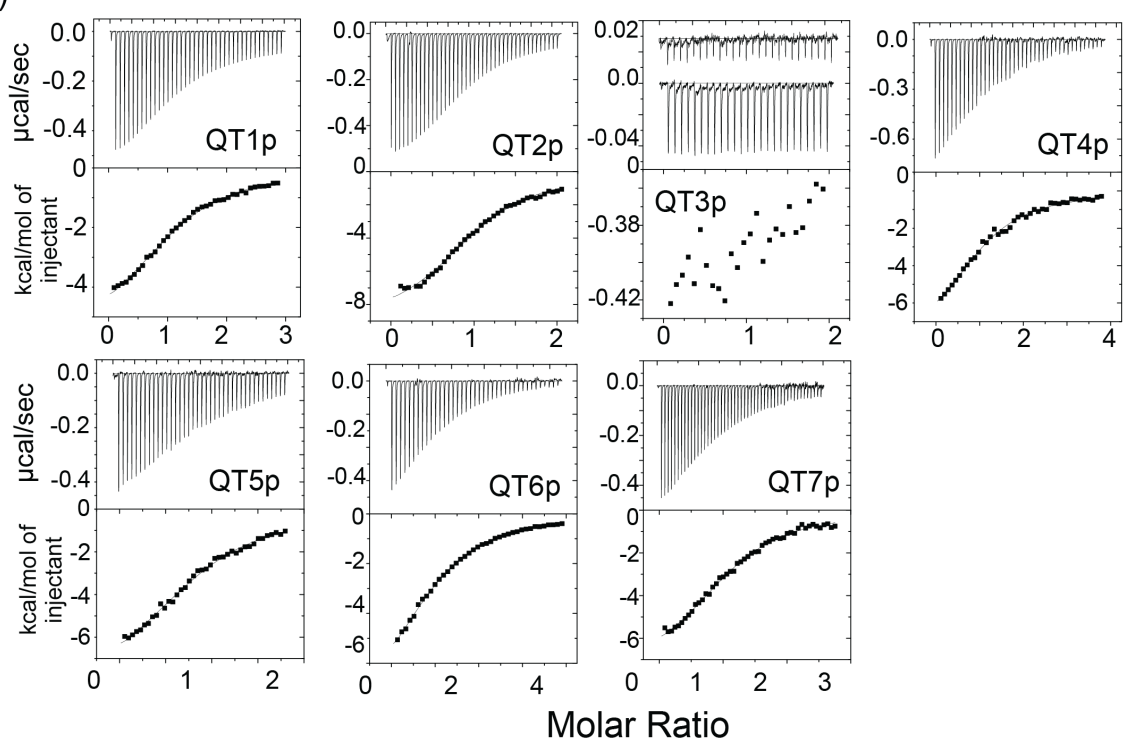

Figure 3. LC8-dLBD interactions monitored by ITC. (a) Construct schematics of the dLBD, QT1-3, QT2-4, QT4-6, and QT4-7 are shown, along with the locations of each TQT motif. A representative isothermal titration calorimetry thermogram of $L C 8$ with the $d L B D$ is shown right. (b) Representative thermograms of LC8 with constructs corresponding to QT1-3, QT2-4, QT4-6, and QT4-7. (b) Representative isothermal titration plots of LC8 with peptides corresponding to QT1p, QT2p, QT3p, QT4p, QT5p, QT6p, and $Q T 7 p$. The interaction with $Q T 3 p$ is too weak to fit to the binding model, so interactions with buffer are plotted on top to show the difference between QT3p interaction with LC8 and buffer. Higher concentrations were not possible due to poor solubility of the QT3p. Data were fit to a single site binding model using Origin software. 
region, indicating a tendency to form transient structure. Heteronuclear NOE values measured at $10^{\circ} \mathrm{C}$ are very low overall, with values ranging from -0.1 to 0.3 , but are also slightly higher for residues $321-363$ (Figure $2 \mathrm{~g}$ ). Together, the $R_{2} / R_{1}$ and heteronuclear NOE values imply that dLBD is highly flexible with transient ordered structure in its C-terminal half.

The presence of transient structure at the C-terminus was validated by generating shorter constructs of the dLBD that include the first three (QT1-3), two internal sets of three (QT2-4 and QT4-6), and last four binding sites (QT4-7)

(Figure 3a). All constructs are of a similar size, varying from 68-84 residues in length. Circular dichroism demonstrates that the C-terminal constructs, QT4-6 and QT4-7, are slightly more ordered than N-terminal constructs, QT1-3 and QT2-4 (Figure 2 - Figure supplement 1a). Size-exclusion chromatography supports this result, as the QT4-6 and QT4-7 constructs elute later, indicating that they are more compact than the $\mathrm{N}$-terminal constructs (Figure 2 - Figure supplement 1b).

\section{Identification and Binding Affinities of LC8 Recognition Motifs in ASCIZ}

Isothermal titration calorimetry (ITC) experiments on dASCIZ constructs were applied to identify the number of recognition motifs and provide estimates of their overall binding affinity for LC8. A range of constructs containing three to seven recognition motifs were tested for their binding to LC8 and all display a single binding step (Figure 3 ). Thus the measured $K_{d}$ and stoichiometry are 'effective' 
values that present an overall simplified picture of a much more complicated complex assembly process.

The full length dLBD binds LC8 with a dLBD:LC8 stoichiometry of 1:7 (two chains of dLBD for 7 LC8 dimers) and an overall $K_{d}$ of $1.4 \mu \mathrm{M}$ (Figure $3 a$, Table 1), suggesting that an additional non-TQT ASCIZ site binds LC8. A plausible candidate is a TMT motif corresponding to residues 285-296 (designated QT3 in Figure 3a). To confirm the functionality of this motif, ITC binding of LC8 was measured for constructs QT1-3, QT2-4, QT4-6, and QT4-7 (Figure 3b). QT1-3 and QT2-4 contain the TMT binding motif, and both bind LC8 with a stoichiometry of 3 , demonstrating that this TMT motif is the seventh LC8 recognition site (Figure 3b, Table 1). Each of the other two constructs bind LC8 with the stoichiometry expected from the number of TQT binding motifs. Interestingly, construct QT4-6, containing recognition sites 4-6, binds LC8 with a $K_{d}$ of $1.0 \mu M$, a slightly higher overall affinity than the full dLBD construct. QT4-7, on the other hand, binds LC8 with a $1.6 \mu \mathrm{M}$ affinity and is slightly more entropically disfavored than either QT4-6 or dLBD (Table 1). The overall $K_{d}$ values of QT1-3, QT2-4, and QT4-7, are 2.4 $\mu \mathrm{M}, 4.1 \mu \mathrm{M}$, and 1.6 $\mu \mathrm{M}$, respectively.

Given the small differences in binding affinity among the shorter dLBD constructs, we asked if any individual site binds LC8 with higher affinity than all others. A series of 14-15 amino acid peptides were synthesized, each 


\begin{tabular}{|c|c|c|c|c|c|}
\hline Construct & $\mathbf{N}$ & Overall $K_{d}(\mu M)$ & $\begin{array}{c}\text { Overall } \Delta H \\
\text { (kcal/mol) }\end{array}$ & $\begin{array}{c}\text { Overall TAS } \\
\text { (kcal/mol) }\end{array}$ & $\begin{array}{r}\text { Overall } \Delta G \\
\text { (kcal } / \mathrm{mol})\end{array}$ \\
\hline dLBD & 7.3 & $1.4 \pm 0.1$ & $-5.3 \pm 0.2$ & $2.7 \pm 0.4$ & $-8.0 \pm 0.4$ \\
\hline QT1-3 (241-324) & 3.2 & $2.4 \pm 0.1$ & $-8.1 \pm 0.4$ & $-0.4 \pm 0.6$ & $-7.7 \pm 0.4$ \\
\hline QT2-4 (271-341) & 2.7 & $4.1 \pm 0.2$ & $-7.9 \pm 0.4$ & $-1.6 \pm 0.5$ & $-6.3 \pm 0.3$ \\
\hline QT4-6 (321-376) & 3.0 & $1.0 \pm 0.1$ & $-10.0 \pm 0.5$ & $-1.8 \pm 0.6$ & $-8.2 \pm 0.4$ \\
\hline QT4-7 (321-388) & 4.0 & $1.6 \pm 0.4$ & $-10.0 \pm 0.5$ & $-2.1 \pm 0.6$ & $-7.9 \pm 0.4$ \\
\hline
\end{tabular}

Table 1. Thermodynamic parameters of dASCIZ-LC8 interactions.

\begin{tabular}{|c|c|c|c|c|c|c|}
\hline Peptide & $\begin{array}{c}\text { Peptide } \\
\text { Sequence }^{\mathrm{a}, \mathrm{b}}\end{array}$ & $\mathbf{N}$ & $\begin{array}{c}\mathrm{K}_{\mathrm{d}} \\
(\mu \mathrm{M})\end{array}$ & $\begin{array}{c}\Delta H \\
\text { (kcal/mol) }\end{array}$ & $\begin{array}{c}\mathrm{T} \Delta \mathrm{S} \\
\text { (kcal/mol) }\end{array}$ & $\underset{(\mathrm{kcal} / \mathrm{mol})}{\Delta G}$ \\
\hline QT1p & yms SQKLDMETQTEe & 1.1 & $\begin{array}{c}14 \pm \\
3.5\end{array}$ & $-5.9 \pm 0.3$ & $0.7 \pm 0.4$ & $-6.6 \pm 0.3$ \\
\hline QT2p & yl apLLRDIETQTPd & 1.0 & $\begin{array}{l}7 \pm \\
0.4\end{array}$ & $-9.2 \pm 0.5$ & $-2.2 \pm 0.6$ & $-7.0 \pm 0.4$ \\
\hline QT3p & ytpdTRGDIGTMTDd & --- & weak & ----- & ----- & ----- \\
\hline QT4p & dlqTSAHMYTQTCd & 1.1 & $\begin{array}{c}15 \pm \\
0.8\end{array}$ & $-8.7 \pm 0.4$ & $-2.1 \pm 0.5$ & $-6.6 \pm 0.3$ \\
\hline QT5p & eelGLSHIQTQTHW & 0.9 & $\begin{array}{l}11 \pm \\
0.6\end{array}$ & $-8.8 \pm 0.4$ & $-2.0 \pm 0.5$ & $-6.8 \pm 0.3$ \\
\hline QT6p & wpdgLYNTQHTQTCd & 1.1 & $\begin{array}{c}20 \pm \\
1.0\end{array}$ & $-8.6 \pm 0.4$ & $-2.2 \pm 0.5$ & $-6.4 \pm 0.3$ \\
\hline QT7p & epdNFQSTCTQTRW & 1.1 & $\begin{array}{c}10 \pm \\
0.5\end{array}$ & $-7.8 \pm 0.4$ & $-0.9 \pm 0.5$ & $-6.9 \pm 0.3$ \\
\hline
\end{tabular}

Table 2. Thermodynamic parameters of peptide-LC8 interactions.

${ }^{a}$ the 10-amino acid LC8 binding motif is capitalized

${ }^{b}$ non-native residues added to the $N$-terminus of each peptide to increase solubility or improve concentration determination are underlined

corresponding to one of the 7 recognition motifs, and their LC8 affinity was measured (Figure 3c, Table 2). The $K_{d}$ values for all peptides indicate surprisingly weak affinity. QT3p interaction with LC8 is not even detected at 30 M LC8. Intriguingly, the QT4-6 construct has the highest binding affinity, while the QT4, QT5, and QT6 peptides display among the lowest affinity as individual 
peptides. These results indicate that positive cooperativity enhances LC8 binding to neighboring recognition motifs.

\section{dLBD and LC8 Form Dynamic, Low-Occupancy Complexes}

Since ITC experiments on the full length dLBD and smaller constructs identify 7 LC8 recognition sites, we sought to establish the size of the dLBD:LC8 complex at varying LC8 concentrations by analytical ultracentrifugation. The dLBD was titrated with increasing concentrations of LC8 and complex formation was assessed at dLBD:LC8 molar ratios 1:1, 1:3, 1:6, and 1:10. Plots of the continuous size distribution, c(S), vs. sedimentation coefficient (Figure 4a) show that titration at sub-saturating concentrations of LC8 results in a broad peak that is likely an equilibrium mixture of complexes with varying LC8 occupancy in exchange with each other and with free dLBD. At a saturating concentration of LC8 (1:10 ratio), a high occupancy complex whose size (7.5 S) approximately corresponds to a fully bound complex $(197 \mathrm{kDa})$ is clearly evident. Contrary to expectations, a low stoichiometry complex ( $5 \mathrm{~S}$, approx. $114 \mathrm{kDa}$ ) is even more highly populated. A high frictional ratio $\left(f / f_{0} \sim 1.6\right)$ indicates an elongated complex, and the molecular weight of this low occupancy complex corresponds roughly to a 1:3 complex, although its broadness, and the approximate nature of the molecular weight determination, indicate that multiple species are present. It is likely that the low occupancy peak, which is roughly twice the intensity of the high occupancy peak, is a heterogeneous mixture of 1:2, 1:3 and 1:4 complexes. 
In summary, while high occupancy complexes are evident in AUC profiles, consistent with the ITC results, these complexes are in equilibrium with many smaller sub-saturated species, the most highly populated of which is a mixture of 1:2-1:4 complexes of dLBD:LC8. The low occupancy complexes are favored, relative to higher occupancy complexes, even in samples having a large excess of LC8.

The presence of stable, low occupancy complexes is supported by small angle X-ray scattering (SAXS) data. A sample composed of dLBD and a large excess of LC8 was injected into an in-line size-exclusion chromatography system and Xray scattering data were collected for the largest peak. Guinier analysis of the data indicates a monodisperse sample suitable for further analysis (Figure 4 Figure supplement 1a). The distance distribution function suggests a moderately compact structure for dLBD:LC8 complexes, with $D_{\max }=240 \AA$, and a molecular weight of roughly $110 \mathrm{kDa}$, suggesting the presence of an unresolved mixture of dLBD:LC8 complexes with stoichiometries ranging from 1:2-1:4 (Figure 4 - Figure supplement $1 \mathrm{~b}$ ). Additionally, a Kratky plot of the scattering data indicates that the dLBD:LC8 complex is a mix of globular domains and intrinsically disordered chains, consistent with low occupancy complex structures (Figure 4 - Figure supplement 1c).

Native gel electrophoresis titration of dLBD with LC8 corroborates the presence of a mixture of complexes (Figure 4b). When unbound, dLBD and LC8 each 
migrate as a single band. When LC8 is added to dLBD at a 1:1 molar ratio, the band for free LC8 disappears, and the complexes formed migrate above LC8, as a diffuse band likely corresponding to two dLBD chains bound to two or more LC8 dimers (since some free dLBD persists). As the molar ratio is increased, the diffuse upper band becomes a dark smear, free LC8 accumulates in a pronounced dark band, and the free dLBD band disappears. We think the most likely explanation is that the decreasing mobility of the upper edge of the smear indicates increasing sizes of the complexes formed. Unbound LC8 is clearly visible at ratios $\geq 1: 4$, indicating that a pool of free LC8 accumulates even at conditions well below LC8 saturation of dLBD.

We similarly assessed the gel mobility of complexes formed by LC8 and the LC8binding domain of human ASCIZ (hLBD), which contains 11 TQT motifs (Rapali, Garcia-Mayoral, et al., 2011) (Figure 1d). Very similar behavior is observed for hLBD, although the gel mobility of hLBD bands is much lower than the mobility of dLBD bands due to molecular sieving of the much larger hLBD (53 kDa) compared to dLBD (17 kDa). Molecular sieving is the dominant effect on hLBD mobility as both hLBD and dLBD are disordered and highly extended and have a pl of $\sim 4$. As LC8 is added to hLBD, the free hLBD band disappears and a lower mobility smear becomes increasingly evident. Decreasing mobility of the upper smear indicates increasing sizes of the complexes formed, while the appearance of a bands migrating the same as free LC 8 at ratios $\geq 1: 4$ indicates the presence of a pool of free LC8 well below LC8 saturation of hLBD. Together the gel 
(a)

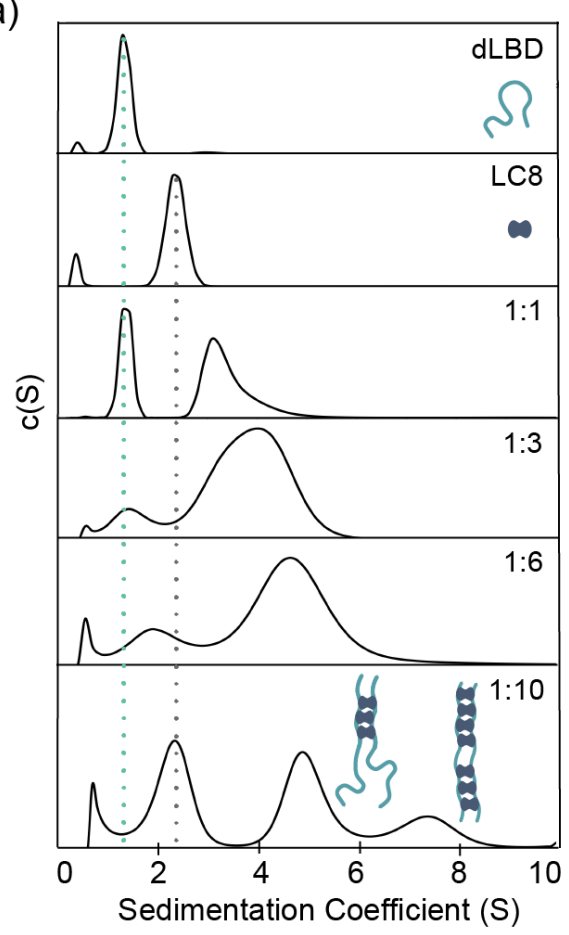

(b)

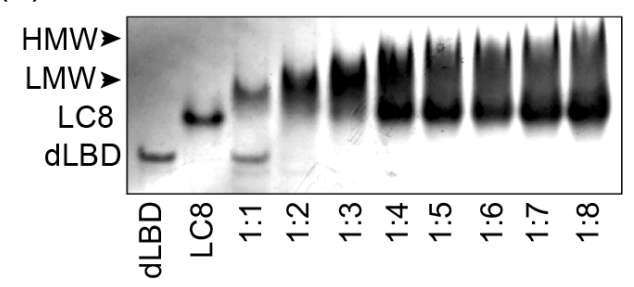

(c)

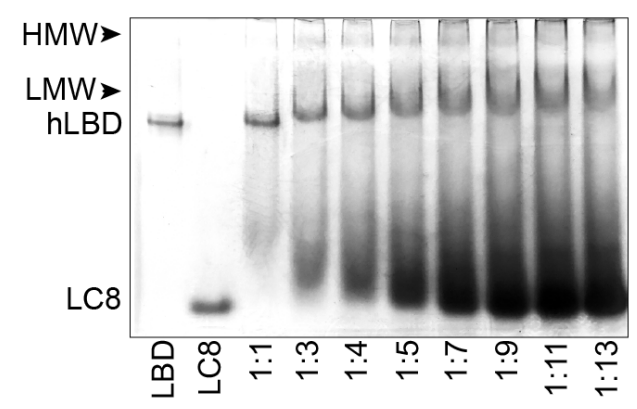

(d)

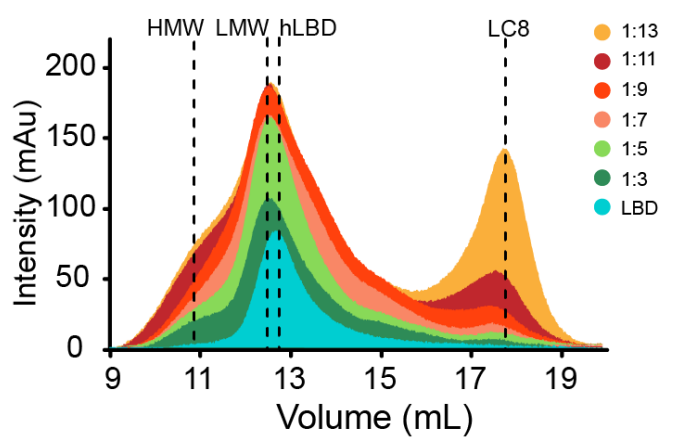

Figure 4. ASCIZ and LC8 form a dynamic complex with low occupancy intermediates. (a) Representative $c(S)$ distributions obtained by sedimentation velocity are shown for the $d L B D, L C 8$, and increasing molar ratios of the $d L B D: \angle C 8 ; 1: 1,1: 3,1: 6$, and 1:10. The standardized sedimentation coefficients $\left(s_{20, w}\right)$ of unbound $L C 8$ and $d L B D$ determined from sedimentation velocity are $2.42 \mathrm{~S}$ (frictional ratio, $f / f_{0}=1.21$, consistent with a globular protein) and $1.34 \mathrm{~S}$ (f// $f_{0}=1.84$, consistent with an asymmetric or unfolded protein), respectively. Calculation of the molecular weight from the sedimentation coefficient and frictional ratio gave masses of $23 \mathrm{kDa}$ and 18 $k D a$ respectively, closely matching their theoretical masses of 24 kDa for LC8 dimer and $17 \mathrm{kDa}$ for dLBD monomer. Cartoon depictions of the ASCIZ dLBD (light blue) and LC8 (dark blue) are shown to aid in visualization of the complexes formed at a 1:10 ratio. (b) Native gel titration of $d L B D$ with LC8. An increasing concentration of $L C 8$ was added to a constant amount of dLBD, from a molar ratio of (dLBD:LC8) 1:1 to 1:8. As more LC8 is added, the complex migrates more slowly and excess LC8 appears at 1:4. (c) Native gel titration of $h L B D$ with $L C 8$. As an increasing concentration of $L C 8$ is added to a constant amount of $h L B D$, Arrows indicate (cont'd page 190) 
titration data for dLBD and hLBD suggest that hLBD:LC8 complexes form a dynamic ensemble with varying levels of LC8 occupancy in which lower occupancy forms are favored. Titration of the hLBD with LC8 by size-exclusion chromatography (Figure 4d) supports our interpretation of the native gel experiments in Figure 4c. Here, the amount of hLBD is held constant, and increases in peak intensity are due to effects on hLBD complexes from increasing LC8. Both LMW and HMW complexes form at the first titration point and increase in population as more LC8 is added. A low occupancy intermediate, labeled LMW, is clearly evident at the lowest molar ratio of $1: 3$, and persists at the same elution volume even at the highest molar ratios. Higher occupancy species, HMW, are also apparent at a ratio of $1: 3$ and become increasingly distinct as LC8 is increased. The peak corresponding to excess LC8 is discernable at a ratio of $1: 7$, and steadily increases as LC8 is added. Taken together, the data in Figure 4 are consistent with the explanation that a low occupancy form of the hLBD:LC8 complex is favored even with a large excess of LC8. A minor population of high occupancy complexes, present even at the lowest molar ratio, increases with increasing LC8 and is in equilibrium with the LMW species and with free LC8, and therefore with each other. Both Drosophila

Figure 4 Cont'd. the locations of the low molecular weight (LMW) and high molecular weight (HMW) complex. (d) Titration of the hLBD with LC8, monitored by size exclusion chromatography on a Superdex 200 gel filtration column. The concentration of $h L B D$ is held constant and an increasing amount of $L C 8$ is added, from a molar ratio of 1:3 to 1:13. Peaks corresponding to free $h L B D$, free $L C 8$, low molecular weight complex (LMW) and high molecular weight $(H M W)$ complex are labeled. Additionally, SAXS data for the LMW dLBD:LC8 complex is shown in figure 4 - figure supplement 1. 
and human LBD exhibit this dynamic behavior, suggesting it is a conserved feature of the ASCIZ:LC8 interaction.

\section{Structure and Distribution of LBD:LC8 Complexes Visualized by Single Particle EM}

In order to visualize the various oligomeric states of ASCIZ-LC8, we analyzed electron microscopy data of dLBD and hLBD under saturating concentrations of LC8. As a positive control, and for validation of EM conditions, similar experiments were carried out with complexes of Nucleoporin159 (Nup159), another intrinsically disordered protein with multiple LC8 binding sites (Stelter et al., 2007). Nup159:LC8 complexes were clearly visualized as a linear array of 5 stacked densities of LC8, as previously reported (Stelter et al., 2007) (data not shown), and consistent with the conclusions of Nup159:LC8 biophysical solution experiments (Nyarko, Song, Novacek, Zidek, \& Barbar, 2013). In contrast, despite the similar overall affinity (Nup159-LC8 $K_{d}=2.9 \mu M$ ) (Nyarko et al., 2013), in negative stain images of dLBD:LC8 and hLBD:LC8 complexes, the vast majority of complex species appear dissociated on the grid (Figure 5a, arrow heads). However, a few observable complexes could be clearly resolved from raw micrographs, identified as linear stacks of punctate densities, akin of beads on a string (Figure 5a, squares), similar to images of Nup159 bound to LC8 (Stelter et al., 2007). Furthermore, although dLBD contains 7 and hLBD 11 LC8 binding sites, the vast majority of complexes observed by EM appeared to be of low LC8 occupancy. 
Reference-free two-dimensional (2D) classification routines were carried out on datasets of $\sim 2000$ single particle images of dLBD:LC8 complexes and $\sim 1000$ particles of hLBD:LC8 complexes extracted from $\sim 300$ and 200 micrographs, respectively. These produced 2D projection averages for dLBD:LC8 and hLBD:LC8 oligomers displaying complexes formed with $2-4$ stacked densities, corresponding to LC8 dimers, deduced from the dimensions of the averaged bead-like densities ( 4 nm diameter) (Benison et al., 2007; Stelter et al., 2007). Complexes with 3 or more LC8 dimers displayed significant conformational flexibility in 2D class averages (Figure $5 \mathrm{~b}$ ) and in the single-particle images (Figure 5a and Figure 5 - Figure Supplement 1). The extent of conformational variability is consistent with $\sim 10-20 \AA$ spacing measured between LC8 densities, and the intrinsic flexibility of the IDP duplex chain separating the neighboring LC8 TQT recognition motifs.

The formation of higher-order oligomers appeared relatively rare, and the fully formed complexes were almost completely absent under saturating LC8 conditions. This low population, coupled with the intrinsic conformational heterogeneity, precluded our ability to obtain 2D class averages of the highoccupancy complexes. To overcome this limitation, statistical analysis describing the distribution of oligomeric states was obtained by hand-selection and classification from single-molecule images (Figure 5 - Figure Supplement 1). Both dLBD:LC8 and hLBD:LC8 complexes form an ensemble of structures, 
displaying an exponential distribution with low-occupancy states (i.e. 2 - 4

stacked LC8 dimers) being most abundant (Figure 5c,d). Density corresponding
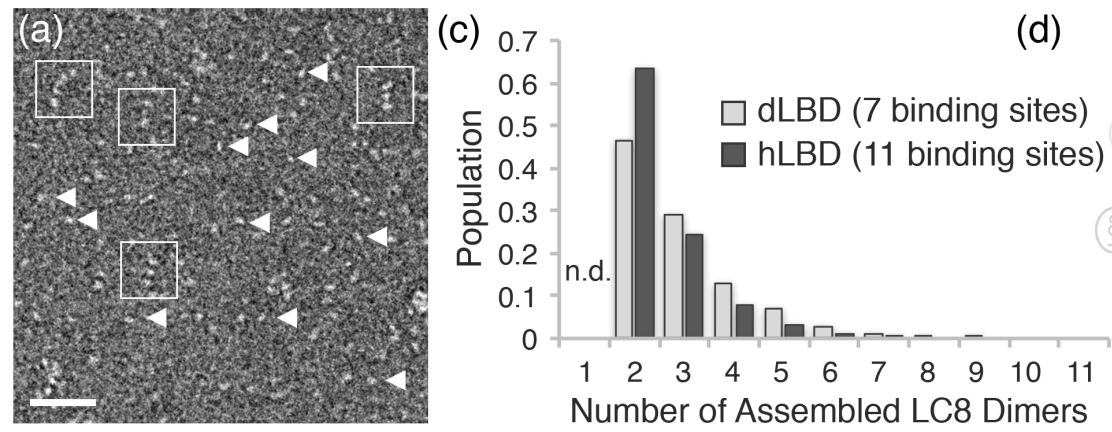

(d)

(b)
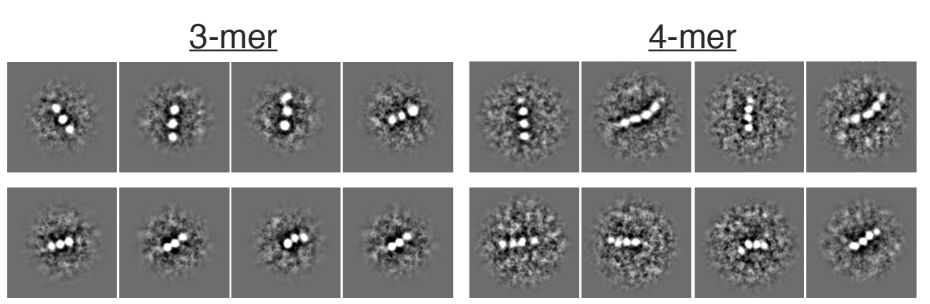

Figure 5. dLBD:LC8 and hLBD:LC8 complexes visualized by negative stain electron microscopy. (a) Representative micrograph of negatively stained hLBD:LC8 complexes. Identified oligomeric complexes are boxed. Non-oligomeric LC8 dimers are indicated by arrowheads. Scale bar $=100 \mathrm{~nm}$. dLBD:LC8 micrographs had the same appearance (not shown). (b) Representative 2D projection averages of (top) dLBD:LC8 oligomers and (bottom) hLBD:LC8 oligomers. Only low-occupancy oligomers with 2 - 4 LC8 dimers (2mer - 4mers) were successfully averaged. Higher-occupancy oligomers were identified in raw micrographs (Figure 5 - figure supplement 1), but were not averaged due to low population and/or high degree of conformational flexibility. Scale bar $=20 \mathrm{~nm}$. (c) Histogram showing the normalized population distribution of LC8 occupancy in complexes formed with $d L B D$ (grey) and hLBD (black), identified from raw micrographs. The population of complexes formed with a single LC8 dimer were not determined (n.d.) (d) Illustration representing the distribution of LC8 occupancy and conformational flexibility observed in hLBD:LC8 complexes.

to the IDP duplex chain cannot be resolved by negative stain EM, therefore complexes formed with a single LC8 dimer were not included in this analysis, as they could not be distinguished from unbound LC8 dimers. 
Together, this analysis shows dLBD and hLBD form dynamic assemblies with LC8 that favor low occupancy states (Figure 5d). Although uncommon, highoccupancy and fully-formed complexes of dLBD:LC8 (1:7 ratio) could be identified from the raw single particle images (Figure 5 - Figure Supplement 1 ), further confirming the stoichiometry obtained by our ITC studies. For the hLBD:LC8 dataset, complexes containing as many as $7-9$ LC8 dimers could be distinguished from the single particle image data, while higher-order complexes containing $10-11$ LC8 dimers were either not distinguishable or were simply absent under the limiting concentrations required for negative stain EM specimen preparation. Nevertheless, the remarkable similarity in distribution of oligomeric species formed by dLBD and hLBD, obtained under similar binding conditions, is consistent with the nearly equivalent overall LC8 affinity determined by ITC, and suggests that a conserved mechanism of negative cooperativity is used by ASCIZ to regulate the formation and distribution of higher-order LC8 assemblies.

\section{NMR Titration of dLBD with LC8 Identifies Sites with Modest Preferential Binding}

If dLBD and LC8 form stable intermediate complexes with excess LC8, which of the seven recognition sites are preferentially bound? Notably, the linear assembly pattern of LC8 dimers observed by negative stain EM suggests an ordered (or quasi-ordered) sequence of assembly, apparently favoring neighboring TQT sites. However, the location of LC8 binding sites could not be 
resolved in these experiments. Therefore, to examine interactions between individual motifs and LC8 in the context of the full dLBD, we turned to NMR. As unlabeled LC8 is titrated into solutions of ${ }^{15} \mathrm{~N}-{ }^{13} \mathrm{C}$ - labeled dLBD, changes in $\mathrm{NH}$ peak intensities can be measured in 3D HNCO spectra recorded for dLBD:LC8 molar ratios of $1: 0.25,1: 1,1: 2,1: 5,1: 8$. As LC8 concentration increases, a corresponding decrease in dLBD peak intensity is observed (Figure 6a). At a molar ratio of $1: 5$, less than $10 \%$ of the original peak intensity remains at all seven LC8 binding sites. At a ratio of 1:8, all dLBD peaks completely disappear except for peaks corresponding to eight $\mathrm{N}$-terminal residues (241-248), indicating that all TQT sites have been occupied to some degree. The absence of peaks for bound dLBD is attributed to line broadening associated with intermediate exchange processes and/or faster transverse relaxation as a result of increased complex size. Therefore, we consider a decrease in peak intensity as a measure of increased complex formation.

Notably, peaks at the C-terminal half of the protein, QT4-7, decrease more quickly than peaks at the $\mathrm{N}$-terminal half, implying that LC8 preferentially occupies these motifs. To confirm this observation and to obtain titration information for the missing residues in this region, we performed similar experiments on the smaller QT2-4 and QT4-6 constructs. QT2-4 was chosen to further validate LC8 binding to the QT3 motif that as an individual peptide showed weak binding by ITC, and QT4-6 was chosen because it has the highest LC8 binding affinity (Figure 3b). Further, the two constructs share the QT4 motif, 
(a)

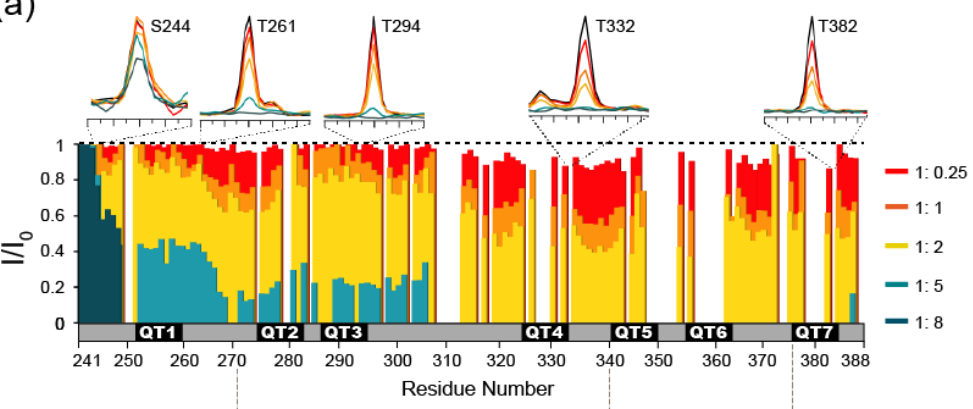

(b)

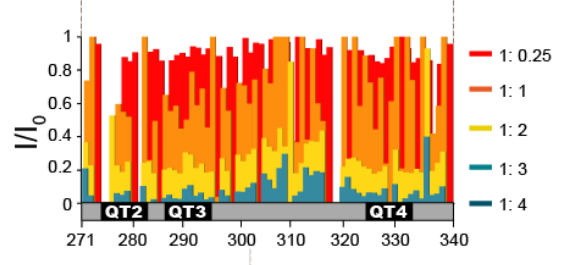

(c) (d)

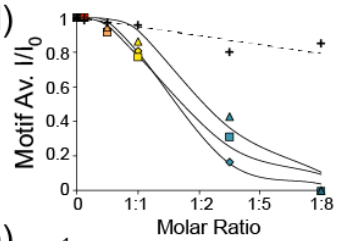

(e)

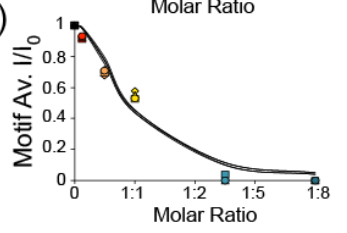

(f)

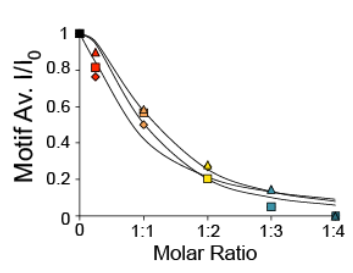

(g)

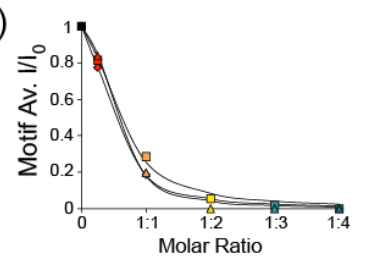

Figure 6. NMR titration of the dLBD with LC8. Relative intensities of nonproline $\mathrm{NH}$ peaks in ${ }^{15} \mathrm{~N}-{ }^{13} \mathrm{C}-H N C O$ spectra are shown for (a) $d L B D$, (b) QT24, and (c) QT4-6 titrated with LC8 at molar ratios of 1:0.25, 1:1, 1:2, 1:5, and 1:8. Peak intensities (I) are given relative to the intensity of the same peak in free peptide (Io). Above (a) are 1D NMR slices of representative amino acids from TQT motifs QT1 (T261), QT3 (T294), QT4 (T332), QT7 (T382) and a control (S244). Representative slices of HNCO spectra from multiple titration points are shown in figure 6 - figure supplement 1 . (d-g) The $1 / I_{0}$ data are alternatively plotted as the average intensity ratio for each 10 -amino acid motif versus the molar ratio of $d L B D: L C 8$. For the $d L B D$ complex, titration curves of individual motifs cluster in two groups having higher (d) or lower (e) average intensity ratios at the same $d L B D: L C 8$ molar ratio. Similarly, for the two shorter constructs, plots of average $I / I_{0}$ for individual motifs cluster in two groups, motifs in QT2-4:LC8 having higher intensity ratios (f), and motifs in QT4-6:LC8 having lower intensity ratios (g). Motif designations in (d) and in (f) are: QT1 (triangle), QT2 (square), QT3 (diamond). Motif designations in (e) and (g) are: QT4 (triangles), QT5 (squares), QT6 (diamond), and QT7 (circle). Note that complete peak attenuation at any titration point is given a value of 0 (Figure 6g). In (d) values are shown for a negative control group (crosses, dotted line) comprised of those measurable at saturating LC8 concentration in the first 8 amino acids, 241-249. In panels (d-g), solid curves are to guide the eye. 
allowing us to assess its affinity in two sequence contexts. Due to the smaller number of peaks for shorter constructs, HSQC spectra have a sufficiently high resolution to render an HNCO-based titration unnecessary. Unlabeled LC8 was titrated into ${ }^{15} \mathrm{~N}$-labeled QT2-4 or QT4-6 and changes in peak intensity were analyzed (Figure 6b,c). As with dLBD, there is a gradual decrease in peak intensity as more LC8 is added to QT2-4 or to QT4-6 at molar ratios: 1:0.25, 1:1, 1:2, 1:3, and 1:4. Significantly, peak intensities in QT4-6 decrease at lower LC8 ratio than in QT2-4, confirming the trend we observe in full-length dLBD. In QT46 , nearly all peaks in the motif region disappear at a ratio of $1: 2$, while $\sim 30 \%$ of peak intensity remains in QT2-4.

Plots of the average peak intensity $\left(1 / I_{0}\right)$ for each 10 -amino acid motif in $d L B D$, and in each of QT2-4 and QT4-6 constructs clearly show the dichotomy in the pattern of peak attenuation (Figure 6d-g). In dLBD, the first three (Figure 6d) show a different titration pattern and weaker binding than the last four motifs (Figure 6e). This dichotomy is replicated in separate plots of the 3 QT motifs in each of the constructs QT2-4 and QT4-6; the average $1 / I_{0}$ of motifs in QT2-4 (Figure 6f) drops to 0.2 at LC8 molar ratio 1:2, twice that observed for motifs in QT4-6 (Figure $6 \mathrm{~g}$ ) which reach the same $\mathrm{I} / \mathrm{I}_{0}$ at molar ratio $1: 1$. The apparently higher LC8 affinity of motifs 4-6 (Figures 6e, g), relative to motifs 2-4 (Figures 6d, f) is consistent with our ITC experiments (Figure 3a). The QT4 motif, common to both constructs, has a different rate of peak disappearance in each construct, suggesting that motif environment, not local sequence, determines its affinity. 
We conclude that the recognition motifs QT4-QT7 are the sites favored in stable low occupancy complexes.

In summary, the data in Figure 6 showing peak attenuation across the whole sequence, even at low LC8 ratios, suggest population of an ensemble with all LC8 sites occupied to varying degree, but with clear preference for the C-terminal motifs. Preferential binding of the C-terminal motifs is additionally supported by ITC results, which show that LC8 binds to the QT4-6 construct with slightly higher affinity than the full-length dLBD (Figure 3a,b). NMR dynamics experiments also demonstrate that residues in the C-terminal motifs are slightly more ordered in comparison to the $\mathrm{N}$-terminal motifs (Figure 2f-g, Figure 2-figure supplement 1a), which may explain the tighter LC8 binding to this region.

\section{Cell-Based Assays Show a Gradient of Transcriptional Activity Modulated by LC8 Binding.}

To investigate how the number of bound LC8 molecules affects the transcriptional activity of $\mathrm{ASClZ}$, we turned our attention to the human protein whose transcriptional activity can be assayed in cell culture using an ASCIZ knockout mouse embryonic fibroblast cell line (Jurado, Conlan, et al., 2012). Human ASCIZ has eleven LC8 recognition motifs that we have numbered 1 to 11 (Figure 7a). To prevent LC8 binding to specific ASCIZ motifs, TQT recognition motifs were mutated to AAA. Five human ASCIZ mutant constructs were 
(a)

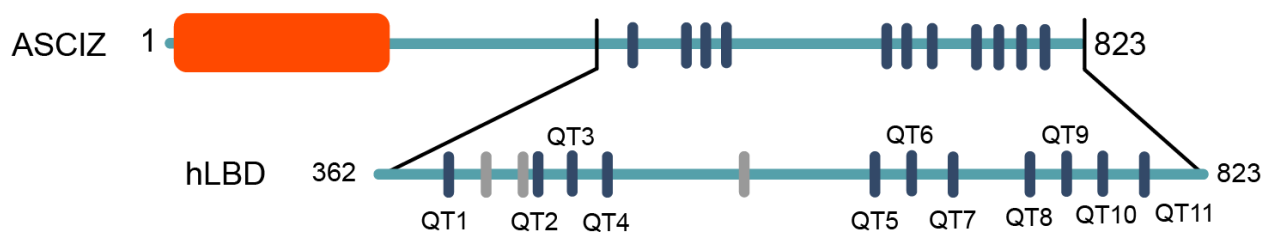

(b)

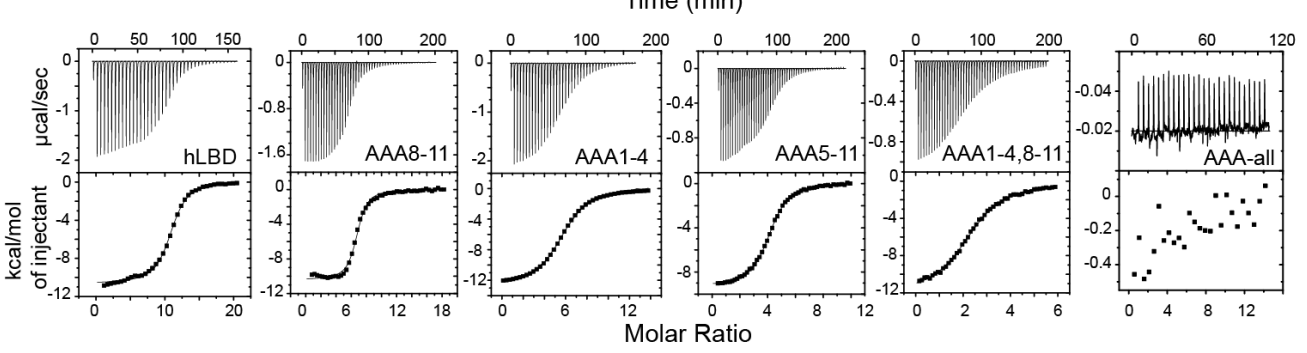

(c)

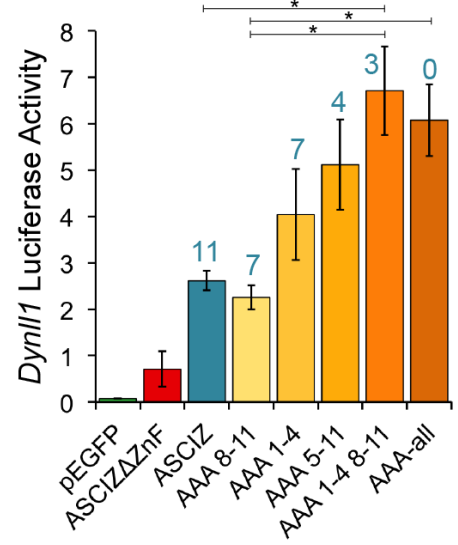

(d)

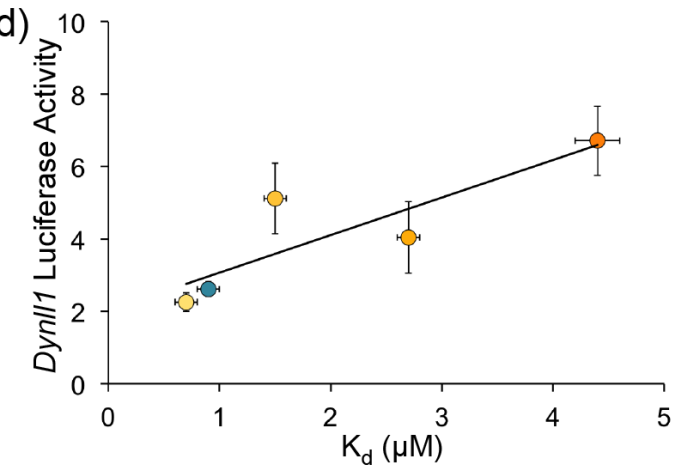

Figure 7. The number of LC8 recognition motifs tunes ASCIZ transcriptional activity. (a) Domain structure of human ASCIZ, showing 11 LC8 binding motifs as blue bars. Additional non-TQT motifs are shown as gray bars. (b) Shown are representative isothermal titration plots of LC8 with the $h L B D$ and constructs with four (AAA1-4 and AAA8-11), seven (AAA5-11), eight (AAA1-4, 8-11), or fourteen (AAA-all) mutant LC8 binding sites. Data were fit to a single site binding model using Origin software. (c) Firefly luciferase reporter assays of ASCIZ knockout mouse embryo fibroblast cells transiently transfected with WT human ASCIZ (blue), a zinc finger deletion construct (red), and ASCIZ mutant constructs AAA1-4, AAA8-11, AAA5-11, $A A A 1-4,8-11$, or $A A A$-all (shades of orange), along with the Dynll1 luciferase and Renilla luciferase vectors. The number of available motifs is indicated above each construct. Error bars are \pm S.E. relative to Renilla luciferase as a control. Asterisks $\left(^{*}\right)$ indicate $p$-values less than 0.01. Data is the average of 24 independent experiments. A western blot depicting expression of the ASCIZ constructs is shown in figure 7 - figure supplement 1. (d) Binding affinity of each construct for LC8 is plotted against luciferase activity. Data points are colored according to (c). The AAA-all construct is excluded from the graph because it does not bind to LC8. 
generated: AAA1-4 with an AAA replacement at each TQT motifs 1-4, and similarly named mutant constructs of AAA8-11; AAA5-11; AAA1-4, 8-11; and AAA-all.

To confirm loss of LC8 binding in vitro for each TQT when replaced with AAA, identical mutations were made in ASCIZ hLBD constructs, named in the same fashion. To completely eliminate LC8 binding in the AAA-all construct, it was necessary to also mutate three SQT/VQT motifs in addition to the 11 TQT motifs identified in our ITC experiments and in pepscan experiments (Figure 7a, gray bars) (Rapali, Garcia-Mayoral, et al., 2011). This suggests that human ASCIZ may contain additional binding motifs, as seen with the TMT motif in Drosophila ASCIZ. The effective affinity and stoichiometry of binding of LC8 to WT ASCIZ hLBD and each mutant hLBD was determined (Figures $7 \mathrm{~b}$, and Table 3). WT hLBD binds LC8 with an hLBD:LC8 ratio of 1:11 (two chains of ASCIZ to 11 LC8 dimers), and an overall $K_{d}$ value of $0.9 \mu \mathrm{M}$ (Table 3). The four hLBD mutants each binds LC8 with the expected stoichiometry for the number of intact LC8 recognition motifs and with overall affinities in the range of $0.7-4.4 \mu \mathrm{M}$.

\begin{tabular}{llllll}
\hline Construct & $\mathbf{N}$ & Overall $\mathrm{K}_{\mathbf{d}}(\boldsymbol{\mu M})$ & $\begin{array}{l}\text { Overall } \Delta \mathbf{H} \\
(\mathbf{k c a l} / \mathrm{mol})\end{array}$ & $\begin{array}{l}\text { Overall } \mathbf{T} \mathbf{S} \\
(\mathbf{k c a l} / \mathrm{mol})\end{array}$ & $\begin{array}{l}\text { Overall } \Delta \mathbf{G} \\
(\mathrm{kcal} / \mathrm{mol})\end{array}$ \\
\hline hLBD & 11.2 & $0.9 \pm 0.1$ & $-10.6 \pm 0.5$ & $-2.4 \pm 0.6$ & $-8.2 \pm 0.4$ \\
\hline AAA8-11 & 6.7 & $0.7 \pm 0.1$ & $-10.4 \pm 0.5$ & $-2.0 \pm 0.6$ & $-8.4 \pm 0.4$ \\
AAA1-4 & 6.6 & $2.7 \pm 0.1$ & $-12.6 \pm 0.6$ & $-5.0 \pm 0.7$ & $-7.6 \pm 0.4$ \\
AAA5-11 & 4.2 & $1.5 \pm 0.1$ & $-9.2 \pm 0.5$ & $-1.3 \pm 0.6$ & $-7.9 \pm 0.4$ \\
AAA1-4, 8-11 & 2.5 & $4.4 \pm 0.2$ & $-12.2 \pm 0.6$ & $-4.9 \pm 0.7$ & $-7.3 \pm 0.4$ \\
\hline
\end{tabular}

Table 3. Thermodynamic parameters of ASCIZ-LC8 interactions. 
To assess the impact of these mutations on ASCIZ transcriptional activity, luciferase reporter assays were carried out using immortalized ASCIZ knockout mouse embryonic fibroblasts transiently transfected with the full-length WT or a mutant $\mathrm{ASClZ}$ gene and a plasmid containing the LC8 promoter. The measured luciferase activity was normalized against Renilla luciferase (Figure 7c). Empty vector and the $\Delta \mathrm{ZnF}$ construct showed limited transcriptional activity compared to ASCIZ constructs. Most significantly, transcriptional activity of the AAA mutants can be ranked to form an activity gradient (Figure 7c) notable for a clear inverse relationship between their transcriptional activity and their affinity for LC8. The construct with the highest affinity for LC8 by ITC, AAA8-11, exhibits equal or slightly lower transcriptional activity than WT ASCIZ, while the construct with the lowest affinity for LC8, AAA1-4, 8-11, has $2.5 x$ the activity of WT ASCIZ. The correlation between transcriptional activity and affinity for LC8 is also shown in Figure $7 \mathrm{~d}$. As the number of available binding sites decreases and $K_{d}$ correspondingly increases, transcriptional activity also increases.

One exception to this trend is the AAA-all construct with zero functional LC8 binding sites (AAA-all), which shows equal or somewhat lower activity than the construct with 3 intact sites (AAA1-4,8-11). As both the ZnF and LBDs of ASCIZ are monomeric in the absence of LC8, a plausible explanation for this effect could be that dimerization of ASCIZ by a minimal number of LC8 molecules is required for optimal binding to the DYNLL1 gene promoter, or the binding of dimeric transcription co-activators to ASCIZ. 
In summary, the data in Figure 7 indicate that, in general, ASCIZ transcriptional activity varies inversely with the number of LC8 recognition motifs and with binding affinity, and that fine tuning within this trend depends on which motifs are occupied and their specific dissociation constants.

\section{Discussion}

A distinctive feature of $A S C I Z$ is the high number of LC8 recognition motifs within its large, disordered C-terminal domain. Here we integrate multiple approaches to elucidate the structure, dynamics, thermodynamics, and hydrodynamics of the large disordered ASCIZ-LC8 complexes that together reveal a new model by which ASCIZ can maintain stable pools of the hub protein LC8. We verified the main features of this model in cells using transcription activity assays which show that mutant ASCIZ constructs with lower LC8 occupancy display higher transcriptional activity, while constructs with higher LC8 occupancy have lower activity. We propose that a dynamic ensemble of complexes is important for finetuning $\mathrm{ASClZ}$ transcriptional activity, where stable, low occupancy complexes function to maintain a basal buffering transcription rate for LC8, while higher occupancy complexes regulated by negative cooperativity switch off transcription. Although many other transcription factors are regulated by multisite phosphorylation (Holmberg, Tran, Eriksson, \& Sistonen, 2002) or multiple binding events to different proteins (Cantor \& Orkin, 2002; Remenyi, Scholer, \& Wilmanns, 2004), we find no examples of activity tuned by multivalent binding to 
the gene product in a negative autoregulatory role, which underscores the novelty and potential impact of this study.

\section{ASCIZ Is a New Type of Multivalent Transcription Factor}

$\mathrm{ASCIZ}$ has three structural and functional features that together set it apart from other multivalent transcription factors. (1) ASCIZ has an exceptionally long intrinsically disordered C-terminal domain compared to other intrinsically disordered transcription factors. Human ASCIZ contains an intrinsically disordered domain that is 600 amino acids long, or $73 \%$ of its overall sequence. The well-studied transcription factor p53, by contrast, is $40 \%$ disordered and the disordered regions are dispersed through three different regions of the protein rather than being concentrated on one terminus (Laptenko, Tong, Manfredi, \& Prives, 2016). Intrinsically disordered regions longer than 50 amino acids are considered to be of significant size for eukaryotic transcription factors (Liu et al., 2006). As intrinsic disorder is proposed to play an important role in regulating function (Shammas, 2017), it is possible that the length of the disordered domain enables a larger diversity of functions. Indeed, recent work has shown that the length of the intrinsically disordered domain can control transcriptional activity through 'energetic frustration', wherein opposing energetic couplings mediate the overall activity (J. Li et al., 2017).

(2) ASCIZ interactions with LC8 display both positive and negative cooperativity that together create a dynamic equilibrium of stable, low occupancy ASCIZ-LC8 
complexes. ASCIZ binding to LC8 forms an IDP duplex scaffold (S. A. Clark et al., 2015) onto which other copies of LC8 or other dimeric partners can bind with higher affinity. The first two to four recognition motifs bind to LC8 with positive cooperativity, as evidenced by ITC experiments that show an enhancement in binding affinity from the presence of neighboring motifs (Figure 3, Table 1). Negative cooperativity regulates the formation and distribution of higher-order LC8 assemblies, as shown by the dominance of low occupancy complexes at saturating concentrations of LC8 (Figure 4,5). Negative cooperativity observed between low occupancy complexes and the fully occupied complex suggests that when the concentration of LC8 exceeds the buffering capacity of the low occupancy intermediates, the fully occupied complex is formed to switch off transcription.

A distribution of low occupancy dynamic complexes is a conserved feature of the ASCIZ:LC8 interaction. Evidence for a dynamic ASCIZ:LC8 ensemble comes from a combination of AUC, gel filtration, native gel electrophoresis, and negative stain electron microscopy data (Figures 4-5, Figure 4 - Figure supplement 1 ). For both Drosophila and human LBD constructs, addition of excess LC8 results in formation of stable low molecular weight (LMW) complexes and a minor population of high molecular weight complexes (Figure 4 and 5). Negative stain electron microscopy experiments show an exponential distribution of complexes, with assemblies containing 2-4 stacked copies of LC8 clearly visualized in 2D projection averages and suggesting a high degree of flexibility within the duplex 
IDP linkers persists upon complex formation (Figure 5).

Multivalency and intrinsic disorder in ASCIZ's LC8 binding domain enable this dynamic ensemble of low occupancy complexes. Many proteins utilize multiple binding sites within intrinsically disordered regions for regulation, complex formation, and a multitude of other functions (Cortese, Uversky, \& Dunker, 2008; Uversky, 2015). In some examples, multiple binding sites serve as a scaffold to bring proteins together (Cortese et al., 2008), while in others, they modulate phase transitions that lead to the formation of bimolecular condensates (Banani, Lee, Hyman, \& Rosen, 2017; Banani et al., 2016). The diversity of these examples highlights the importance of multivalency and intrinsically disordered regions in protein function and regulation. It is of note that although ASCIZ'S multiple binding sites are similar to those that lead to phase transitions in other systems (P. Li et al., 2012), we did not detect this behavior in vitro. However, ASCIZ puncta formation has been observed in cell culture in response to treatment with MMS, a DNA methylation agent (Jurado, Conlan, et al., 2012; Jurado et al., 2010)' (McNees, Conlan, Tenis, \& Heierhorst, 2005). These puncta do not form in the absence of LC8, indicating that LC8 binding to multiple recognition motifs is necessary for foci formation.

Comparison of ASCIZ with another LC8 multivalent binding partner, Nup159, underscores the uniqueness of the ASCIZ-LC8 assembly. While three other multivalent LC8 binding partners with more than two recognition motifs are 
known to exist (Dunsch et al., 2012; Fejtova et al., 2009; Gupta et al., 2012; Stelter et al., 2007), the role of multiple sites has only been characterized for ASCIZ (this work) and Nup159 (Nyarko et al., 2013). Nup159 cooperatively binds five LC8 dimers and forms a relatively stable complex readily visible by electron microscopy and 2D classification analysis (Stelter et al., 2007). As Nup159 has a slightly lower affinity for LC8 than ASCIZ $(2.9 \mu \mathrm{M}$ vs. $0.9 \mu \mathrm{M}$, respectively ((Nyarko et al., 2013)), the difference between the Nup159 grids with uniformly stacked structures and the ASCIZ grids with sparse and heterogeneous structures of 2-4 stacked LC8 dimers is intriguing. This difference in structural heterogeneity can be attributed to the higher flexibility or short life time of ASCIZ:LC8 complexes relative to Nup159:LC8 complexes, while the scarcity of high-occupancy states is consistent with a unique mode of negative cooperativity. Given the different function of Nup159 (in nuclear pore assembly) versus ASCIZ:LC8 complexes (transcription regulation), the data suggest that the dynamic properties and unique mechanism of assembly that is conserved in LC8-ASCIZ complexes may reflect an important feature required for autoregulation of LC8 transcription.

(3) ASCIZ tunes its transcriptional activity by binding multiple copies of its gene product, LC8. Cell culture based transcription assays demonstrate that ASCIZ affinity for LC8 is negatively correlated with transcriptional activity (Figure 7). When fewer LC8 binding motifs are occupied and ASCIZ affinity for LC8 is reduced, transcription is increased, and vice versa. 
A great example of multisite regulation is the E26 transformation-specific transcription factor (Ets-1), which tunes its transcriptional activity through multisite phosphorylation of its serine-rich domain. Phosphorylation of the serine rich region occludes the DNA-binding interface and stabilizes its helical inhibitory module, inhibiting Ets-1 DNA binding 20-fold (Desjardins et al., 2014; G. M. Lee et al., 2008; Pufall et al., 2005). Similarly, the function of p53 is modulated by over 50 posttranslational modifications that are proposed to be interdependent (Meek \& Anderson, 2009). Phosphorylation of specific p53 residues prevents binding to the inhibitory protein HDM2, while increasing binding to the activating proteins CREB-binding protein (CBP) and p300 (Ferreon et al., 2009). p53 affinity for CBP/p300 depends on the extent of p53 phosphorylation; successive phosphorylation events increase p53 affinity for the TAZ1, TAZ2, and KIX domains of CBP/p300 (C. W. Lee, Ferreon, Ferreon, Arai, \& Wright, 2010; Teufel, Bycroft, \& Fersht, 2009). p53 also binds to a multitude of other proteins that regulate its activity (Beckerman \& Prives, 2010). While many other transcription factors tune their activity through multisite regulation, we could find no other examples besides ASCIZ where binding to multiple copies of their gene product modulates activity; yet, the prevalence of IDP domains in transcription factors indicates that such mechanisms are likely to be widespread and studies such as these are becoming more tractable with the integrated approaches used here. 


\section{Model of ASCIZ Transcriptional Regulation}

Based on our experimental data, we have developed a model of ASCIZ transcriptional regulation that illustrates the relationship between transcriptional activity (red arrow) and LC8 concentration (blue arrow) (Figure 8). As the cellular level of LC8 (blue dimers) increases, the number of LC8 molecules bound to ASCIZ also increases. The ASCIZ-LC8 complex primarily exists as a dynamic equilibrium of different complex stoichiometries and degree of disorder (center brackets). We propose that this low occupancy conformational ensemble is important for maintaining a basal level of LC8 transcription. It acts as a buffer for changes in the concentration of LC8 and fine-tunes transcription levels according

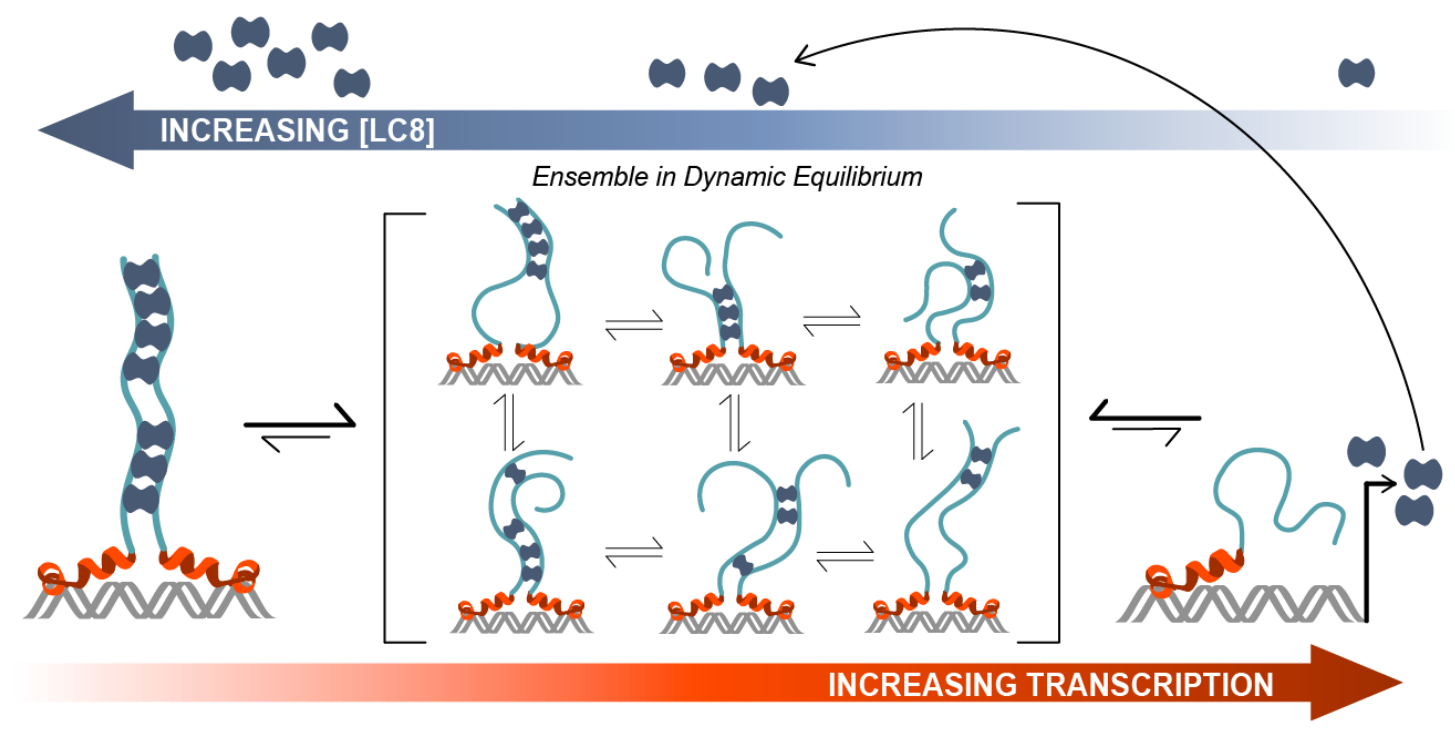

Figure 8. Model of ASCIZ regulation of LC8 transcription. A proposed model of LC8 transcriptional regulation is shown for $d A S C I Z$, which also applies to the human protein. Free LC8 dimers (dark blue) bind to ASCIZ and modulate transcriptional activity. Transcriptional activity is lowest when ASCIZ recognition motifs are fully occupied by $L C 8$ (left) and is highest when ASCIZ is bound to a small number of LC8 dimers (right). A dynamic, low-occupancy complexes (center) are composed of ASCIZ bound to 2-4 LC8 dimers. LC8 that is produced upon ASCIZ transcription returns to the pool of free $L C 8$. 
to cellular needs. Negative cooperativity observed between low occupancy complexes and the fully occupied complex suggests that when the concentration of LC8 exceeds the buffering capacity, the fully occupied complex is formed to switch off transcription. As LC8 is a hub protein that interacts with $>40$ protein partners, and is predicted to bind to 100 additional proteins from a diverse selection of cellular pathways (Rapali, Radnai, et al., 2011), maintaining a certain level of its transcription is essential. 


\section{Materials and Methods}

\section{Cloning, Protein Expression, and Purification}

Studies were carried out using constructs from human ASCIZ (Uniprot O43313) as well as Drosophila ASCIZ (dASCIZ) (Uniprot Q9VZU1) which, with its fewer recognition motifs, smaller size, and available mutant phenotypes, is a tractable model of the human ASCIZ. Constructs of the dASCIZ zinc finger domain (ZnF) and the LC8 binding domain (dLBD) were generated by cloning residues 1-156 or 241-388, respectively, of Drosophila ASCIZ into the pET2Zt2-1a vector. The constructs were expressed in frame with a hexahistidine tag, Protein A solubility tag, and cleavage site for the tobacco etch virus (TEV) enzyme. Shorter constructs of the dLBD were generated by cloning residues 241-324 (QT1-3), 271-341 (QT2-4), 299-376 (QT4-6), and 321-388 (QT4-7) into the pET2Zt2-1a vector. The human LC8 binding domain (hLBD) construct was generated by cloning human ASCIZ (Uniprot O43313) residues 362-823 into the pET24d vector (Novagen) and expressing the construct in frame with a hexahistidine tag and TEV cleavage site.

For the five human ASCIZ mutants, ASCIZ AAA1-4, AAA8-11, AAA5-11, AAA14,8-11, and AAA-all, residues 7-9 of the LC8 binding motif (usually the residues TQT), were mutated to AAA to prevent binding. Mutations were performed using either the QuikChange Lightening Mutagenesis Kit (Agilent) or by synthesizing short constructs (300-350 bp) containing the desired mutations and using Gibson Assembly (New England Biosciences) to insert them into the LC8-binding domain 
(hLBD) gene (residues $362-823$ of human ASCIZ). All constructs were transformed into Escherichia coli Rosetta DE3 cells and expressed at $37^{\circ} \mathrm{C}$ in LB or minimal autoinduction media with ${ }^{12} \mathrm{C}$ or ${ }^{13} \mathrm{C}$ glycerol and ${ }^{15} \mathrm{NH}_{4} \mathrm{Cl}$ as the sole carbon and nitrogen sources, respectively. Recombinant protein expression was induced with $0.4 \mathrm{mM} \mathrm{IPTG} \mathrm{(for} \mathrm{LB} \mathrm{cultures)} \mathrm{and} \mathrm{growth} \mathrm{continued} \mathrm{at} 25^{\circ} \mathrm{C}$ for 16 hours. Cells were harvested and purified under denaturing conditions using TALON His-Tag Purification protocol (Clontech). The solubility tag and/or hexahistidine tag were cleaved by TEV protease and the protein was further purified using strong anion exchange chromatography (Bio-Rad) followed by gel filtration on a Superdex ${ }^{\mathrm{TM}} 75$ gel filtration column (GE Health). The purity of the recombinant proteins, as assessed by SDS-polyacrylamide gels, was $>95 \%$. The pure proteins were stored at $4^{\circ} \mathrm{C}$ and used within 1 week. LC8 was prepared as previously described (Barbar et al., 2001).

\section{Peptide Design and Synthesis}

Peptides corresponding to the seven putative recognition sequences from dASCIZ were commercially synthesized: YMSSQKLDMETQTEE (QT1p), $\begin{array}{llll}\text { YLAPLLRDIETQTPD } & \text { (QT2p), } & \text { YTPDTRGDIGTMTDD } & \text { (QT3p), }\end{array}$ DLQTSAHMYTQTCD (QT4p), EELGLSHIQTQTHW (QT5p), WPDGLYNTQHTQTCD (QT6p), and EPDNFQSTCTQTRW (QT7p) (GenScript, Piscataway, NJ). Non-native amino acids (underlined in Table 2) were added to the N-terminus to enhance solubility or concentration determination by UV absorbance at $280 \mathrm{~nm}$. 


\section{Isothermal Titration Calorimetry}

Binding thermodynamics of the ASCIZ and dASCIZ construct/peptide-LC8 interactions were obtained at $25^{\circ} \mathrm{C}$ with a VP-ITC microcalorimeter (Microcal). The binding buffer was composed of $50 \mathrm{mM}$ sodium phosphate, $50 \mathrm{mM}$ sodium chloride, $1 \mathrm{mM}$ sodium azide, $5 \mathrm{mM} \beta$-mercaptoethanol, $\mathrm{pH}$ 7.5. dLBD was placed in the reaction cell at a concentration of $8 \mu \mathrm{M}$ and titrated with LC8 at a concentration of $800 \mu \mathrm{M}$. For binding of dASCIZ constructs QT1-3, QT2-4, QT46, and QT4-7, $10 \mu \mathrm{M}$ of construct was titrated with $400 \mu \mathrm{M} \mathrm{LC8}$. For interactions with synthetic peptide, peptides were dissolved in binding buffer to a final concentration of $300 \mu \mathrm{M}$ and then added to LC8 at a concentration of $30 \mu \mathrm{M}$ in the reaction cell. ASCIZ hLBD and mutant hLBD constructs were placed in the reaction cell at a concentration of 9-16 $\mu \mathrm{M}$ and titrated with $900 \mu \mathrm{M}$ LC8. Peak areas were integrated and data were fit to a single-site binding model in Origin 7.0 from which the stoichiometry $(N)$, dissociation constant $\left(K_{d}\right)$, and the change in enthalpy $(\Delta H)$, and entropy $(\Delta S)$ were obtained. Reported data are the average of two or more independent experiments. As the binding-model fit was very good and data were reproducible, error was determined based on a $5 \%$ uncertainty in protein concentration calculations.

\section{Circular Dichroism}

CD experiments were conducted on a Jasco720 spectropolarimeter in a $1 \mathrm{~mm}$ cell. For the spectrum of dLBD and smaller constructs, ten scans were averaged 
at a concentration of $30 \mu \mathrm{M}$ in a buffer composed of $10 \mathrm{mM}$ sodium phosphate, $\mathrm{pH} 7.5$, at $25^{\circ} \mathrm{C}$ and $10^{\circ} \mathrm{C}$. For the $\mathrm{ZnF}$, ten scans were averaged at a concentration of $25 \mu \mathrm{M}$ in a buffer composed of $10 \mathrm{mM}$ sodium phosphate, 200 $\mathrm{mM}$ sodium sulfate, $50 \mu \mathrm{M}$ zinc sulfate, $\mathrm{pH} 7.5$, at $10^{\circ} \mathrm{C}, 25^{\circ} \mathrm{C}$, and $35^{\circ} \mathrm{C}$.

\section{Analytical Ultracentrifugation}

Sedimentation velocity experiments for the titration of dLBD and LC8 were performed in a Beckman Coulter Model XL-I analytical ultracentrifuge equipped with UV/Vis scanning optics. Reference (400 $\mu \mathrm{L}$ binding buffer; $50 \mathrm{mM}$ sodium phosphate, $50 \mathrm{mM}$ sodium chloride, $1 \mathrm{mM}$ sodium azide, $5 \mathrm{mM}$ TCEP, $\mathrm{pH}$ 7.5) and sample $(380 \mu \mathrm{L})$ solutions were loaded into $12 \mathrm{~mm}$ double-sector cells with quartz windows and the cells were then mounted in an An-50 Ti 8-hole rotor. LC8 was prepared at a concentration of $15 \mu \mathrm{M}$ while the concentration of dLBD was varied from $15-1.5 \mu \mathrm{M}$. Proteins were centrifuged at $50,000 \mathrm{rpm}$ at $20^{\circ} \mathrm{C}$, and radial absorbance data were collected at appropriate wavelengths in continuous mode every 5 minutes without averaging. Data were fit to a continuous sizedistribution $[c(S)]$ model using the program SEDFIT (Schuck, 2000). The partial specific volume of the proteins, buffer density, and buffer viscosity were computed using the program SEDNTERP (Hayes, 1995).

Sedimentation velocity experiments for the $\mathrm{ZnF}$ domain were performed on a Beckman ProteomeLab ${ }^{\mathrm{TM}} \mathrm{XL}-\mathrm{A} / \mathrm{XL}-\mathrm{I}$ analytical ultracentrifuge in a buffer composed of $50 \mathrm{mM}$ sodium phosphate, $200 \mathrm{mM}$ sodium chloride, $0.4 \mathrm{mM}$ zinc 
sulfate, $1 \mathrm{mM}$ sodium azide, $2 \mathrm{mM}$ TCEP, $\mathrm{pH}$ 7.0. The sample was centrifuged at $40,000 \mathrm{rpm}$ at $20^{\circ} \mathrm{C}$ for 7 hours and absorbance data were collected at $286 \mathrm{~nm}$. Data were fit to a continuous size-distribution [c(s)] model using the program SEDPHAT (Vistica et al., 2004).

\section{NMR Experiments}

NMR measurements were collected at $10^{\circ} \mathrm{C}$, using $300-350 \mu \mathrm{M}$ isotopically $\left({ }^{13} \mathrm{C} /{ }^{15} \mathrm{~N}\right.$ or $\left.{ }^{15} \mathrm{~N}\right)$ labeled dLBD in a buffer at $\mathrm{pH} 6.5$ composed of $10 \mathrm{mM}$ sodium phosphate, $10 \mathrm{mM}$ sodium chloride, $1 \mathrm{mM}$ sodium azide, $10 \mathrm{mM} \beta$ mercaptoethanol, a protease inhibitor mixture (Roche Applied Science), and 2-2 dimethylsilapentane-5-sulfonic acid for ${ }^{1} \mathrm{H}$ chemical shifts referencing. Data for backbone assignments were collected on a Bruker Avance $850 \mathrm{MHz}$ spectrometer equipped with a cryoprobe. Five-dimensional $\mathrm{HN}(\mathrm{CA}) \mathrm{CONH}$ and HabCabCONH experiments (Motackova et al., 2010; Novacek et al., 2011) and a three-dimensional HNCO experiment were acquired with non-uniform sampling of the indirectly detected dimensions and used for sequential assignment of ${ }^{13} \mathrm{C}$ ${ }^{15} \mathrm{~N}-\mathrm{dLBD}$.

Interaction of unlabeled LC8 and ${ }^{13} \mathrm{C}-{ }^{15} \mathrm{~N}$ labeled dLBD was characterized by collecting three-dimensional BEST-TROSY-HNCO spectra at multiple molar ratios of LC8, 1: 0.25 (dLBD: LC8), 1:1, 1:2, 1:5, and 1:8. For the interaction of unlabeled LC8 with ${ }^{15} \mathrm{~N}$-labeled QT2-4 (residues 271-341) or QT4-6 (residues 321-376), two-dimensional BEST-TROSY-HSQC spectra were collected at the 
molar ratios (QT2-4/QT4-6:LC8) 1:0.25, 1:1, 1:2, 1:3, and 1:4. NMR titration data were analyzed and plotted by measuring peak volumes using Sparky and averaging over each 10 amino acid QT motif.

HNCO-based $R_{1}$ relaxation measurements experiments were recorded with relaxation delay times ranging from 11.2 to $2352 \mathrm{~ms}$, and the $R_{2}$ relaxation data were acquired using relaxation delays ranging from 14.4 to $259 \mathrm{~ms}$. Sixteen total $R_{1}$ or $R_{2}$ experiments were recorded, including six duplicate experiments for error determination. Curve fitting was performed using the rate analysis script Sparky2Rate and the program Curvefit (A. G. Palmer, Columbia University). Steady-state ${ }^{1} \mathrm{H}-{ }^{15} \mathrm{~N}$ heteronuclear NOEs were acquired using $6 \mathrm{~s}$ total saturation time. Error bars were determined from the intensities of the baseline noise using the formula $\sigma /(\mathrm{NOE})=\left[\left(\sigma \mathrm{I}_{\text {sat }} / \mathrm{I}_{\text {sat }}\right)^{2}+\left(\sigma \mathrm{l}_{\text {unsat }} / l_{\text {unsat }}\right)^{2}\right]^{1 / 2}$, where $\mathrm{I}_{\text {sat }}$ and $\left.\sigma\right|_{\text {sat }}$ correspond to the intensity of the peak and its baseline noise.

All two-dimensional spectra and the three-dimensional HNCO spectra were processed using TopSpin (Bruker Biosciences), and the non- uniformly sampled five-dimensional $\mathrm{HN}(\mathrm{CA}) \mathrm{CONH}$ and $\mathrm{HabCabCONH}$ spectra were processed with Sparse Multidimensional Fourier Transform (the software for data processing is available online at the Warsaw University Laboratory (nmr.cent3.uw.edu.pl/software)). All spectra were analyzed with the graphical NMR assignment and integration software Sparky 3.115. 


\section{Analytical Size Exclusion Chromatography and Native Gel Titration}

hLBD at a concentration of $30 \mu \mathrm{M}$ was incubated with $600 \mu \mathrm{M} \mathrm{LC8}$ at various molar ratios: (hLBD:LC8) 1:3, 1:5, 1:7, 1:9, 1:11, and 1:13. The complex was loaded on a Superdex 200 analytical column (GE healthcare) in binding buffer: $50 \mathrm{mM}$ sodium phosphate, $50 \mathrm{mM} \mathrm{NaCl}, 5 \mathrm{mM} \beta$-mercaptoethanol, $1 \mathrm{mM}$ sodium azide, $\mathrm{pH}$ 7.5. 100 or $200 \mu \mathrm{l}$ of protein samples were injected at a flow rate of 0.5 $\mathrm{ml} / \mathrm{min}$ at room temperature and samples were monitored by UV absorption at $280 \mathrm{~nm}$.

For native gel electrophoresis titrations, dLBD or hLBD and LC8 were incubated at the molar ratios listed above and run on a $10 \%$ polyacrylamide gel at a constant 10 mAmps for 5-7 hours.

\section{Small Angle X-ray Scattering}

Small-angle X-ray scattering experiments were conducted at the ESRF BioSAXS beamline BM29 (Pernot et al., 2013) in Grenoble, France. dLBD and LC8 samples were purified as described above and dialyzed into binding buffer (50 $\mathrm{mM}$ sodium phosphate, $50 \mathrm{mM}$ sodium chloride, $10 \mathrm{mM}$ beta-mercaptoethanol, 1 $\mathrm{mM}$ sodium azide, $\mathrm{pH}$ 7.5) before SAXS measurements. $30 \mu \mathrm{l}$ of dLBD:LC8 complex (1:8 molar ratio) at five different concentrations for each sample (and buffer) were exposed to X-rays and scattering data collected using the robotic sample handling available at the beamline. 10 individual frames were collected for every exposure, each 2 seconds in duration using the Pilatus $1 \mathrm{M}$ detector 
(Dectris). Individual frames were processed automatically and independently within the EDNA framework, yielding individual radially averaged curves of normalized intensity versus scattering angle $s=4 \pi \operatorname{Sin} \theta / \lambda$. Additional data reduction within EDNA utilizes the automatic data processing tools of EMBLHamburg ATSAS package (Petoukhov, 2007), to combine timeframes, excluding any data points affected by aggregation induced by radiation damage, yielding the average scattering curve for each exposure series. Matched buffer measurements taken before and after every sample were averaged and used for background subtraction. Merging of separate concentrations and further analysis steps were performed manually using the tools of the ATSAS package (Petoukhov, 2007). The forward scattering I(0) radius of gyration, Rg were calculated from the Guinier approximation (A., 1938), the hydrated particle volume was computed using the Porod invariant (Porod, 1982) and the maximum particle size $D_{\max }$, was determined from the pair distribution function computed by GNOM (Svergun, 1992) using PRIMUS.

\section{Electron Microscopy}

Electron microscopy (EM) studies were conducted using dLBD and hLBD peptides incubated with a molar excess of LC8, and the formed complexes were negatively stained for contrast enhancement using established protocols (Myers et al., 2017). Briefly, dLBD (50 nM) was mixed with LC8 at a molar ratio 1:8, and human hLBD peptide $(50 \mathrm{~nm})$ was mixed with LC8 at a molar ratio of 1:13, in EM buffer containing $20 \mathrm{mM}$ Tris, $\mathrm{pH}$ 7.5, $50 \mathrm{mM} \mathrm{NaCl}, 10 \mathrm{mM} \mathrm{BME}$ and $1 \mathrm{mM} \mathrm{NaN}$. 
A $3 \mu \mathrm{l}$ drop of sample was applied to a glow-discharged continuous carbon coated EM specimen grid (400 mesh Cu grid, Ted Pella). Excess protein was removed by blotting with filter paper and washing the grid two times with EM buffer. The specimen was then stained with freshly prepared $0.75 \%$ (wt vol ${ }^{-1}$ ) uranyl formate (SPI-Chem).

Negatively stained specimens were visualized on a $120 \mathrm{kV}$ TEM (iCorr, FEI) at a nominal magnification of $49,000 x$ at the specimen level. Digital micrographs were recorded on a $2 \mathrm{~K} \times 2 \mathrm{~K} \mathrm{CCD}$ camera (FEl Eagle) with a calibrated pixel size of $4.37 \AA$ pixel $^{-1}$ and a defocus of $2.0-3.5$ um. For the dLBD-LC8 specimen, a total of 2,574 single particle images were extracted from $\sim 300$ micrographs, and for human dLBD-LC8 1,234 particles were extracted from 200 micrographs. Complexes with clear oligomeric structure could be identified and were handselected using EMAN2 (Tang et al., 2007). Single particle images were extracted with a box size of $160 \times 160$ pixels and CTF-corrected (phase-flipped) in EMAN2. Reference-free 2D class averages were generated in EMAN2 and RELION 2.0 (Scheres, 2012) using CTF-corrected and high-pass filtered image datasets. Statistical analysis of oligomeric composition for both datasets was performed by counting the number of subunits identified from single particle images and classifying them manually as $2-7$ mers (dLBD:LC8 complexes) or $2-11$ mers (hLBD:LC8 complexes) (Figure 5 - Figure Supplement 1). Particles that could not be confidently assigned were discarded, leaving 2,334 oligomers assigned for the dLBD:LC8 dataset and 967 for human hLBD:LC8 dataset. Complexes 
containing only a single LC8 dimer could not be distinguished from unbound LC8 particles, and were not included in our analysis.

As a positive control, Nucleoporin159 (Nup159) in complex with LC8 was also prepared for negative stain EM under similar conditions to the $\mathrm{dLBD} / \mathrm{hLBD}$ samples, and as previously described (Stelter et al., 2007) (not shown). As a negative control, we prepared EM grids with LC8 alone and dLBD/hLBD peptides alone. No oligomeric structures (i.e. beads on a string) were observed in these images (not shown).

\section{Transcription Reporter Assays}

To measure transcriptional activity of $\mathrm{ASClZ}$ mutants, six ASCIZ constructs were cloned into the pEGFP vector (Clontech): WT ASCIZ (1-823), $\triangle \mathrm{ZnF}$ (230-823), ASCIZ AAA1-4, ASCIZ AAA8-11, ASCIZ AAA5-11, and ASCIZ AAA1-4, 8-11. Approximately $2 \mathrm{kbp}$ of the Dynll1 promoter was cloned into the pGL3 vector (Promega) upstream of the firefly luciferase gene as previously described (Jurado, Conlan, et al., 2012). Using FuGENE 6 (Promega), immortalized ASCIZ KO MEFs (Jurado et al., 2010) were co-transfected with ASCIZ constructs, the Dynll1 promoter, and a pRL-CMV vector containing Renilla luciferase for normalization of firefly/luciferase ratios. 24 hours after transfection, cells were transferred to 96-well plates and incubated overnight before determining reporter

gene activities using the dual-luciferase reporter assay kit (Promega) and a Polarstar Optima (BMG Labtechnologies) instrument. For assessment of protein 
expression levels, human U2OS cells were transfected with ASCIZ constructs using FuGENE 6 and were probed with ASCIZ antibody (McNees et al., 2005).

\section{BMRB Accession Code}

The chemical shifts for dLBD ASCIZ have been deposited in the Biological Magnetic Resonance Data Bank under accession code 27412.

\section{Acknowledgements}

The authors are indebted to Professor Clare Woodward for the many helpful discussions. This work was supported by National Institutes of Health Grant GM 084276 to E.B. Support to facilities includes the Oregon State University NMR Facility funded in part by the National Institutes of Health, HEI Grant 1S10OD018518, and by the M. J. Murdock Charitable Trust grant \# 2014162. Access to the NMR facility of CEITEC Masaryk University was provided by iNEXT, project number 653706, funded by the Horizon 2020 programme of the European Union. This article reflects only the author's view and the European Commission is not responsible for any use that may be made of the information it contains. JH was supported by the National Health and Medical Research Council of Australia (Senior Research Fellowship APP1022469 and Project Grant APP1026125) and Victorian State Government Operational Infrastructure Support. Electron microscopy was conducted with support from the Multiscale Microscopy Core (OHSU), Advanced Computing Center (OHSU) and the 
National Institutes of Health Grant R35GM124779 to SLR. Small angle X-ray scattering data were collected at the European Molecular Biology Lab in Grenoble, France.

\section{List of Figure Supplements}

Figure 2 - figure supplement 1

Figure 4 - figure supplement 1

Figure 5 - figure supplement 1

Figure 6 - figure supplement 1

Figure 7 - figure supplement 1 
(a)

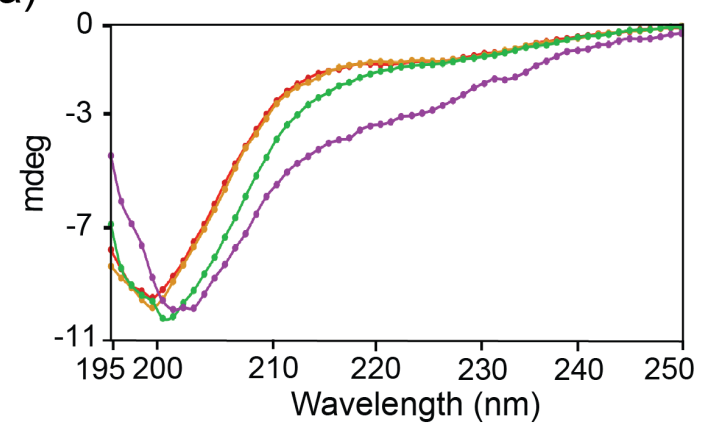

(b)

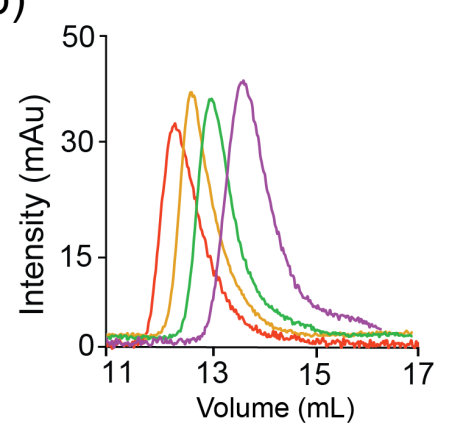

Figure 2 - Figure supplement 1. dLBD C-terminus is transiently structured. (a) Far UV CD spectrum of dLBD constructs collected at $10{ }^{\circ} \mathrm{C}$ : QT1-3 (red), QT2-4 (orange), QT4-6 (green), and QT4-7 (magenta). (b) Size exclusion chromatography of each construct from (a) depicts differences in retention time, using the same color scheme. Size exclusion chromatography was performed on a Superdex 75 10/300 column.

(a)

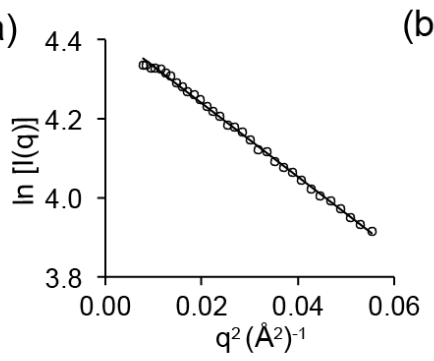

(b)

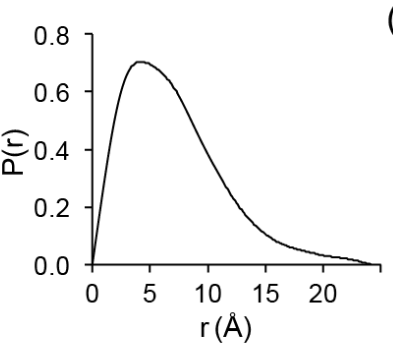

(c)

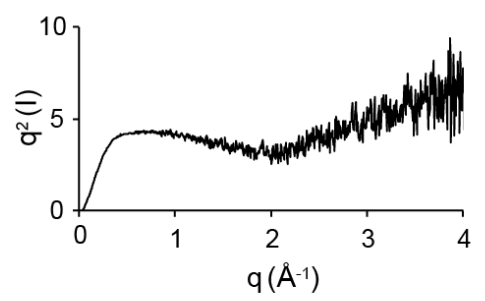

Figure 4 - Figure supplement 1. Primary SAXS data. (a) A Guinier plot of the experimental scattering data is shown. The solid line represents the Guinier fit, where the linear fit was extended to $q<1.3 / R_{g}$ (Kikhney \& Svergun, 2015). (b) The real-space pairwise distribution function for the dLBD:LC8 complex. (c) Kratky plot of the dLBD:LC8 complex. 


\section{(a)}
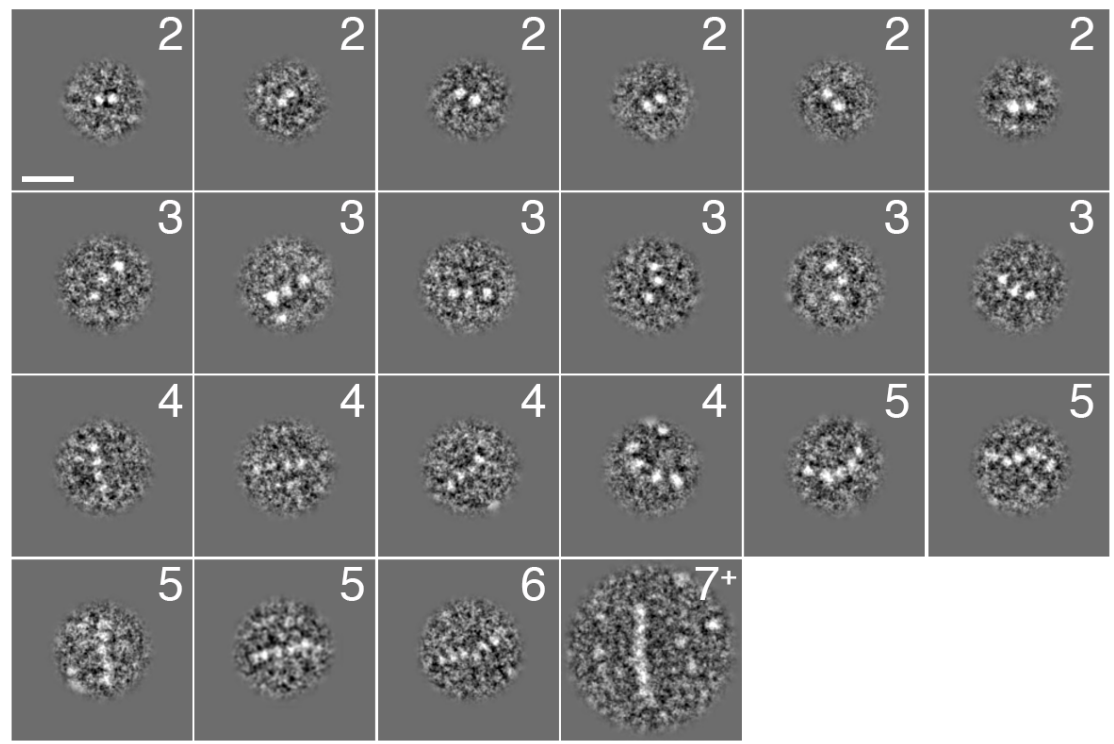

(b)

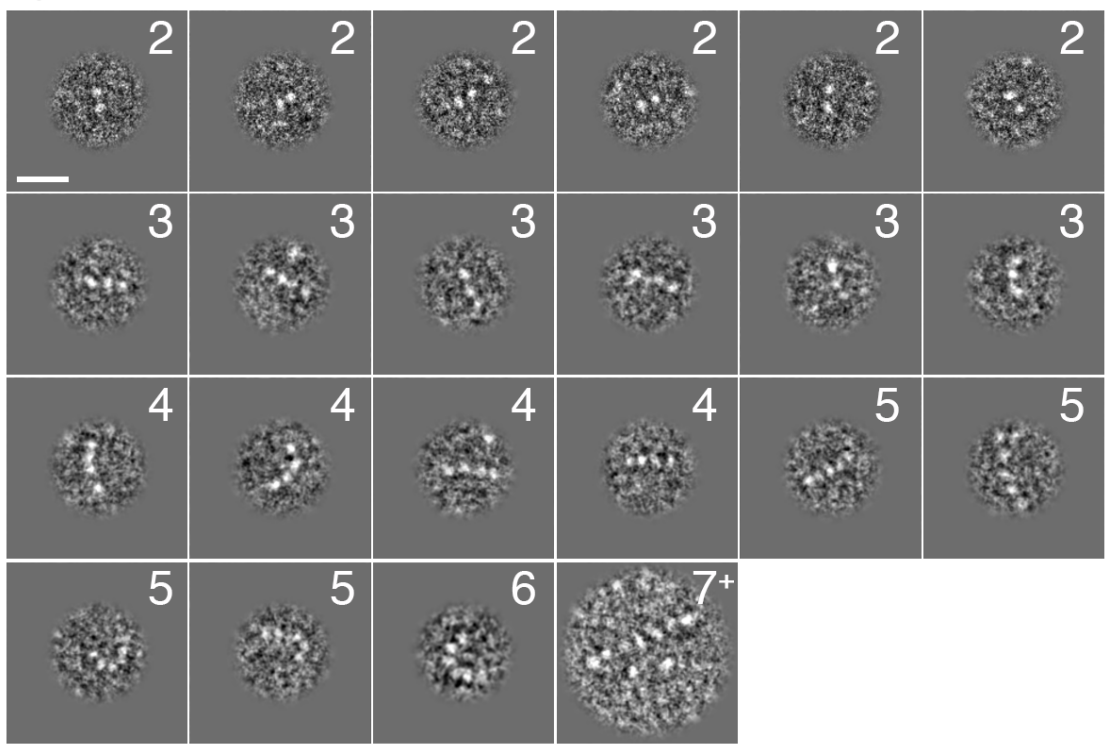

Figure 5 - Figure supplement 1. Single particle images of dLBD:LC8 and hLBD:LC8 complexes. (a) Representative images of negatively stained (a) dLBD:LC8 complexes and (b) hLBD:LC8 complexes extracted from raw micrographs. Each particle is annotated to indicate the assigned occupancy of LC8 dimers used for statistical analysis in Figure 5. The classification of 7+ in panels $a$ and $b$, indicates the possibility of a higher-order structure that is not fully resolved. Scale bars $=20 \mathrm{~nm}$. 

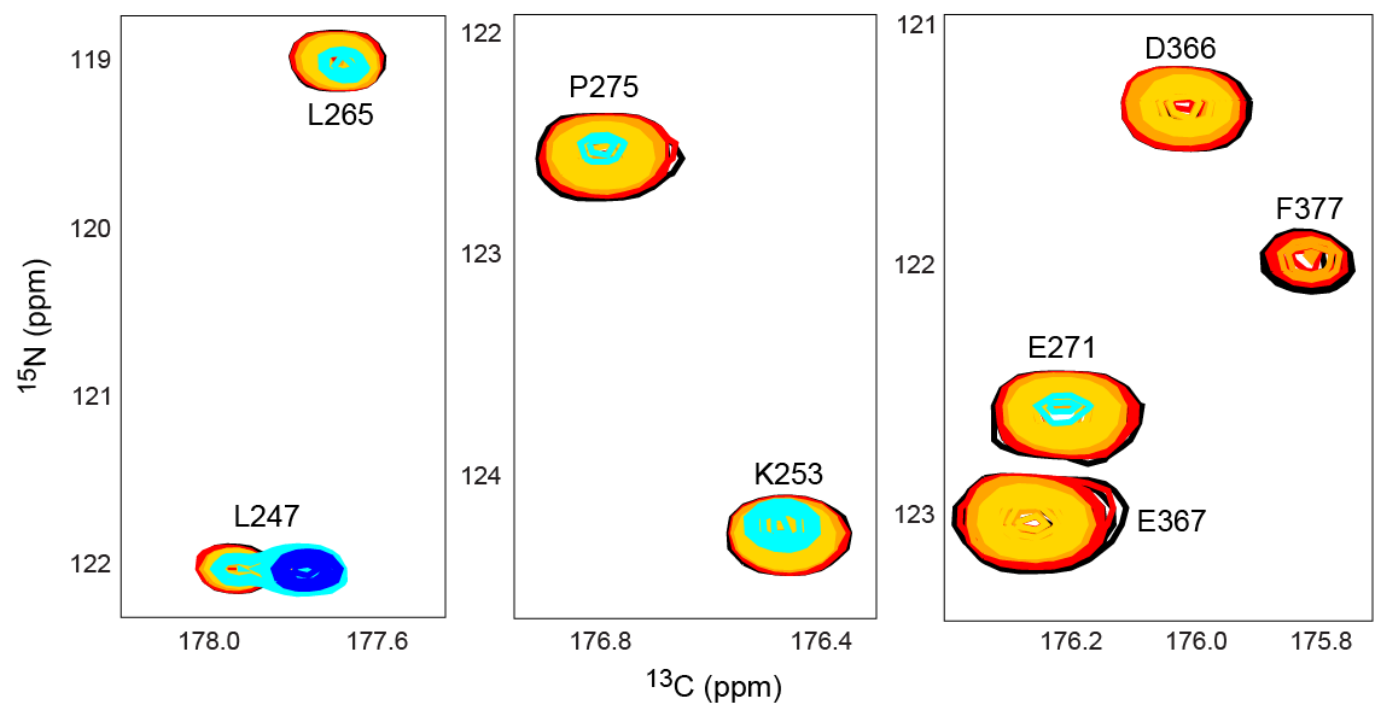

Figure 6 - Figure supplement 1. Representative HNCO slices of dLBD titration with LC8. Shown are representative peaks from HNCO spectra depicting a loss of intensity with an increasing LC8 concentration. Colors are the same as Figure 6a: free dSQTQ (black), 1 LBD: 0.25 LC8 (red), 1:1 (orange), 1:2 (gold), 1:5 (blue), and 1:8 (dark blue).

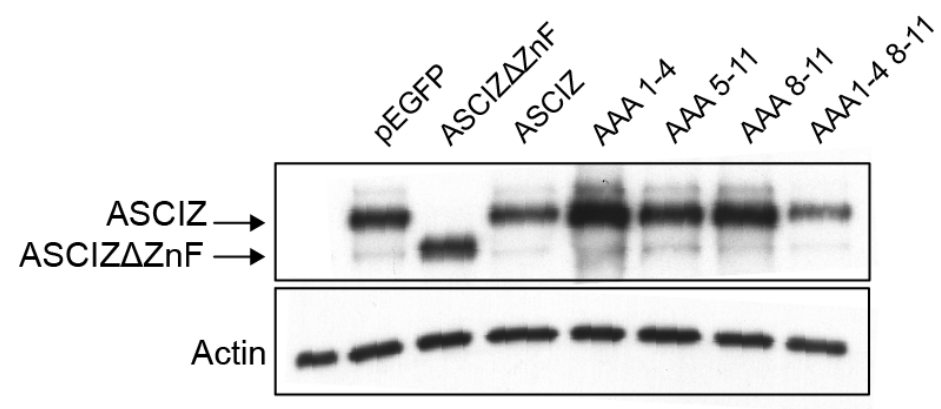

Figure 7 - Figure supplement 1. Western blot of ASCIZ constructs. Western blot analysis of human U2OS cells transiently transfected with ASCIZ constructs from (Figure 7c), probed with ASCIZ antibody. 


\section{References Cited}

A., G. (1938). The diffusion of X-rays under the extremely weak angles applied to the study of fine particles and colloidal suspension. Comptes Rendus Hebdomadaires Des Seances De L Acad Des Sci., 206, 1374-1376.

Banani, S. F., Lee, H. O., Hyman, A. A., \& Rosen, M. K. (2017). Biomolecular condensates: organizers of cellular biochemistry. Nat Rev Mol Cell Biol, 18(5), 285-298. doi: 10.1038/nrm.2017.7

Banani, S. F., Rice, A. M., Peeples, W. B., Lin, Y., Jain, S., Parker, R., \& Rosen, M. K. (2016). Compositional Control of Phase-Separated Cellular Bodies. Cell, 166(3), 651-663. doi: 10.1016/j.cell.2016.06.010

Banerjee, N. a. Z. M. (2003). Identifying cooperativity among transcription factors controlling the cell cycle in yeast. Nucleic Acids Res., 31(23), 7024-70311.

Barbar, E. (2008). Dynein light chain LC8 is a dimerization hub essential in diverse protein networks. Biochemistry, 47(2), 503-508. doi: $10.1021 / \mathrm{bi} 701995 \mathrm{~m}$

Barbar, E., Kleinman, B., Imhoff, D., Li, M., Hays, T. S., \& Hare, M. (2001). Dimerization and folding of LC8, a highly conserved light chain of cytoplasmic dynein. Biochemistry, 40(6), 1596-1605.

Barbar, E., \& Nyarko, A. (2014). NMR Characterization of Self-Association Domains Promoted by Interactions with LC8 Hub Protein. Comput Struct Biotechnol J, 9, e201402003. doi: 10.5936/csbj.201402003

Beckerman, R., \& Prives, C. (2010). Transcriptional regulation by p53. Cold Spring Harb Perspect Biol, 2(8), a000935. doi: 
10.1101/cshperspect.a000935

Benison, G., Karplus, P. A., \& Barbar, E. (2007). Structure and dynamics of LC8 complexes with KXTQT-motif peptides: swallow and dynein intermediate chain compete for a common site. J Mol Biol, 371(2), 457-468. doi: 10.1016/j.jmb.2007.05.046

Boratyn, G. M., Camacho, C., Cooper, P. S., Coulouris, G., Fong, A., Ma, N., . . . Zaretskaya, I. (2013). BLAST: a more efficient report with usability improvements. Nucleic Acids Res, 41(Web Server issue), W29-33. doi: 10.1093/nar/gkt282

Cantor, A. B., \& Orkin, S. H. (2002). Transcriptional regulation of erythropoiesis: an affair involving multiple partners. Oncogene, 21(21), 3368-3376. doi: 10.1038/sj.onc.1205326

Chintapalli, V. R., Wang, J., \& Dow, J. A. (2007). Using FlyAtlas to identify better Drosophila melanogaster models of human disease. Nat Genet, 39(6), 715-720. doi: $10.1038 / \mathrm{ng} 2049$

Clark, S., Nyarko, A., Lohr, F., Karplus, P. A., \& Barbar, E. (2016). The Anchored Flexibility Model in LC8 Motif Recognition: Insights from the Chica Complex. Biochemistry, 55(1), 199-209. doi: 10.1021/acs.biochem.5b01099

Clark, S. A., Jespersen, N., Woodward, C., \& Barbar, E. (2015). Multivalent IDP assemblies: Unique properties of LC8-associated, IDP duplex scaffolds. FEBS Lett, 589(19 Pt A), 2543-2551. doi: 10.1016/j.febslet.2015.07.032

Cortese, M. S., Uversky, V. N., \& Dunker, A. K. (2008). Intrinsic disorder in 
scaffold proteins: getting more from less. Prog Biophys Mol Biol, 98(1), 85106. doi: 10.1016/j.pbiomolbio.2008.05.007

Currie, S. L., Doane, J. J., Evans, K. S., Bhachech, N., Madison, B. J., Lau, D. K. W., . . Graves, B. J. (2017). ETV4 and AP1 Transcription Factors Form Multivalent Interactions with three Sites on the MED25 ActivatorInteracting Domain. J Mol Biol, 429(20), 2975-2995. doi: 10.1016/j.jmb.2017.06.024

Desjardins, G., Meeker, C. A., Bhachech, N., Currie, S. L., Okon, M., Graves, B. J., \& McIntosh, L. P. (2014). Synergy of aromatic residues and phosphoserines within the intrinsically disordered DNA-binding inhibitory elements of the Ets-1 transcription factor. Proc Natl Acad Sci U S A, 111(30), 11019-11024. doi: 10.1073/pnas.1401891111

Dunsch, A. K., Hammond, D., Lloyd, J., Schermelleh, L., Gruneberg, U., \& Barr, F. A. (2012). Dynein light chain 1 and a spindle-associated adaptor promote dynein asymmetry and spindle orientation. J Cell Biol, 198(6), 1039-1054. doi: 10.1083/jcb.201202112

Dyson, H. J., \& Wright, P. E. (2016). Role of Intrinsic Protein Disorder in the Function and Interactions of the Transcriptional Coactivators CREBbinding Protein (CBP) and p300. J Biol Chem, 291(13), 6714-6722. doi: 10.1074/jbc.R115.692020

Ezomo, O. F., Kazuya Takahashi, Yuki Horie, Mohammed S. Mustak and Shunsuke Meshitsuka. (2010). Circular Dichroism Studies on C-terminal Zinc Finger Domain of Transcription Factor GATA-2. Yonago Acta medica, 
53(53), 25-28.

Fejtova, A., Davydova, D., Bischof, F., Lazarevic, V., Altrock, W. D., Romorini, S., . . Gundelfinger, E. D. (2009). Dynein light chain regulates axonal trafficking and synaptic levels of Bassoon. J Cell Biol, 185(2), 341-355. doi: $10.1083 / j c b .200807155$

Ferreon, J. C., Lee, C. W., Arai, M., Martinez-Yamout, M. A., Dyson, H. J., \& Wright, P. E. (2009). Cooperative regulation of p53 by modulation of ternary complex formation with CBP/p300 and HDM2. Proc Natl Acad Sci U S A, 106(16), 6591-6596. doi: 10.1073/pnas.0811023106

Goggolidou, P., Hadjirin, N. F., Bak, A., Papakrivopoulou, E., Hilton, H., Norris, D. P., \& Dean, C. H. (2014). Atmin mediates kidney morphogenesis by modulating Wnt signaling. Hum Mol Genet, 23(20), 5303-5316. doi: $10.1093 / \mathrm{hmg} / \mathrm{ddu} 246$

Goggolidou, P., Stevens, J. L., Agueci, F., Keynton, J., Wheway, G., Grimes, D. T., . . Norris, D. P. (2014). ATMIN is a transcriptional regulator of both lung morphogenesis and ciliogenesis. Development, 141(20), 3966-3977. doi: 10.1242/dev.107755

Gupta, A., Diener, D. R., Sivadas, P., Rosenbaum, J. L., \& Yang, P. (2012). The versatile molecular complex component LC8 promotes several distinct steps of flagellar assembly. J Cell Biol, 198(1), 115-126. doi: 10.1083/jcb.201111041

Hall, J., Karplus, P. A., \& Barbar, E. (2009). Multivalency in the assembly of intrinsically disordered Dynein intermediate chain. J Biol Chem, 284(48), 
33115-33121. doi: 10.1074/jbc.M109.048587

Hayes, D. L., T.; Philo, J. (1995). Program Sednterp: Sedimentation Interpretation Program. Alliance Protein Laboratories.

Holmberg, C. I., Tran, S. E., Eriksson, J. E., \& Sistonen, L. (2002). Multisite phosphorylation provides sophisticated regulation of transcription factors. Trends Biochem Sci, 27(12), 619-627.

Jurado, S., Conlan, L. A., Baker, E. K., Ng, J. L., Tenis, N., Hoch, N. C., . . . Heierhorst, J. (2012). ATM substrate Chk2-interacting Zn2+ finger (ASCIZ) Is a bi-functional transcriptional activator and feedback sensor in the regulation of dynein light chain (DYNLL1) expression. J Biol Chem, 287(5), 3156-3164. doi: 10.1074/jbc.M111.306019

Jurado, S., Gleeson, K., O'Donnell, K., Izon, D. J., Walkley, C. R., Strasser, A., . . . Heierhorst, J. (2012). The Zinc-finger protein ASCIZ regulates B cell development via DYNLL1 and Bim. J Exp Med, 209(9), 1629-1639. doi: 10.1084/jem.20120785

Jurado, S., Smyth, I., van Denderen, B., Tenis, N., Hammet, A., Hewitt, K., . . . Heierhorst, J. (2010). Dual functions of ASCIZ in the DNA base damage response and pulmonary organogenesis. PLoS Genet, 6(10), e1001170. doi: 10.1371/journal.pgen.1001170

Kidane, A. I., Song, Y., Nyarko, A., Hall, J., Hare, M., Lohr, F., \& Barbar, E. (2013). Structural features of LC8-induced self-association of swallow. Biochemistry, 52(35), 6011-6020. doi: 10.1021/bi400642u

Kikhney, A. G., \& Svergun, D. I. (2015). A practical guide to small angle X-ray 
scattering (SAXS) of flexible and intrinsically disordered proteins. FEBS Lett, 589(19 Pt A), 2570-2577. doi: 10.1016/j.febslet.2015.08.027

King, A., Li, L., Wong, D. M., Liu, R., Bamford, R., Strasser, A., . . Heierhorst, J. (2017). Dynein light chain regulates adaptive and innate B cell development by distinctive genetic mechanisms. PLoS Genet, 13(9), e1007010. doi: 10.1371/journal.pgen.1007010

Laptenko, O., Tong, D. R., Manfredi, J., \& Prives, C. (2016). The Tail That Wags the Dog: How the Disordered C-Terminal Domain Controls the Transcriptional Activities of the p53 Tumor-Suppressor Protein. Trends Biochem Sci, 41(12), 1022-1034. doi: 10.1016/j.tibs.2016.08.011

Lee, C. W., Ferreon, J. C., Ferreon, A. C., Arai, M., \& Wright, P. E. (2010). Graded enhancement of p53 binding to CREB-binding protein (CBP) by multisite phosphorylation. Proc Natl Acad Sci U S A, 107(45), 1929019295. doi: 10.1073/pnas.1013078107

Lee, G. M., Pufall, M. A., Meeker, C. A., Kang, H. S., Graves, B. J., \& Mclntosh, L. P. (2008). The affinity of Ets-1 for DNA is modulated by phosphorylation through transient interactions of an unstructured region. J Mol Biol, 382(4), 1014-1030. doi: 10.1016/j.jmb.2008.07.064

Li, J., White, J. T., Saavedra, H., Wrabl, J. O., Motlagh, H. N., Liu, K., . . Hilser, V. J. (2017). Genetically tunable frustration controls allostery in an intrinsically disordered transcription factor. Elife, 6. doi: 10.7554/eLife.30688

Li, P., Banjade, S., Cheng, H. C., Kim, S., Chen, B., Guo, L., . . Rosen, M. K. 
(2012). Phase transitions in the assembly of multivalent signalling proteins. Nature, 483(7389), 336-340. doi: 10.1038/nature10879

Liang, J., Jaffrey, S. R., Guo, W., Snyder, S. H., \& Clardy, J. (1999). Structure of the PIN/LC8 dimer with a bound peptide. Nat Struct Biol, 6(8), 735-740. doi: $10.1038 / 11501$

Liu, J., Perumal, N. B., Oldfield, C. J., Su, E. W., Uversky, V. N., \& Dunker, A. K. (2006). Intrinsic disorder in transcription factors. Biochemistry, 45(22), 6873-6888. doi: 10.1021/bi0602718

McGuffin, L. J., Bryson, K., \& Jones, D. T. (2000). The PSIPRED protein structure prediction server. Bioinformatics, 16(4), 404-405.

McNees, C. J., Conlan, L. A., Tenis, N., \& Heierhorst, J. (2005). ASCIZ regulates lesion-specific Rad51 focus formation and apoptosis after methylating DNA damage. EMBO J, 24(13), 2447-2457. doi: 10.1038/sj.emboj.7600704

Meek, D. W., \& Anderson, C. W. (2009). Posttranslational modification of p53: cooperative integrators of function. Cold Spring Harb Perspect Biol, 1(6), a000950. doi: 10.1101/cshperspect.a000950

Minezaki, Y., Homma, K., Kinjo, A. R., \& Nishikawa, K. (2006). Human transcription factors contain a high fraction of intrinsically disordered regions essential for transcriptional regulation. J Mol Biol, 359(4), 11371149. doi: 10.1016/j.jmb.2006.04.016

Motackova, V., Novacek, J., Zawadzka-Kazimierczuk, A., Kazimierczuk, K., Zidek, L., Sanderova, H., . . Sklenar, V. (2010). Strategy for complete 
NMR assignment of disordered proteins with highly repetitive sequences based on resolution-enhanced 5D experiments. J Biomol NMR, 48(3), 169-177. doi: 10.1007/s10858-010-9447-3

Myers, J. B., Zaegel, V., Coultrap, S. J., Miller, A. P., Bayer, K. U., \& Reichow, S. L. (2017). The CaMKII holoenzyme structure in activation-competent conformations. Nat Commun, 8, 15742. doi: 10.1038/ncomms15742

Novacek, J., Zawadzka-Kazimierczuk, A., Papouskova, V., Zidek, L., Sanderova, H., Krasny, L., . . Sklenar, V. (2011). 5D 13C-detected experiments for backbone assignment of unstructured proteins with a very low signal dispersion. J Biomol NMR, 50(1), 1-11. doi: 10.1007/s10858-011-9496-2

Nyarko, A., Song, Y., Novacek, J., Zidek, L., \& Barbar, E. (2013). Multiple recognition motifs in nucleoporin Nup159 provide a stable and rigid Nup159-Dyn2 assembly. J Biol Chem, 288(4), 2614-2622. doi: 10.1074/jbc.M112.432831

Pernot, P., Round, A., Barrett, R., De Maria Antolinos, A., Gobbo, A., Gordon, E., . . McSweeney, S. (2013). Upgraded ESRF BM29 beamline for SAXS on macromolecules in solution. J Synchrotron Radiat, 20(Pt 4), 660-664. doi: $10.1107 / S 0909049513010431$

Petoukhov, M. V. K., P. V.; Kikhney A. G. ; Svergun D. I. (2007). ATSAS 2.1 towards automated and web-supported small-angle scattering data analysis. J. Appl. Cryst., 40, s223-s228.

Porod, G. (1982). General Theory. In K. O. Glatter O (Ed.), Small angel X-ray scattering (pp. 17-51). London: Academic Press. 
Pufall, M. A., Lee, G. M., Nelson, M. L., Kang, H. S., Velyvis, A., Kay, L. E., . . . Graves, B. J. (2005). Variable control of Ets-1 DNA binding by multiple phosphates in an unstructured region. Science, 309(5731), 142-145. doi: 10.1126/science.1111915

Puthalakath, H., Huang, D. C., O'Reilly, L. A., King, S. M., \& Strasser, A. (1999). The proapoptotic activity of the Bcl-2 family member Bim is regulated by interaction with the dynein motor complex. Mol Cell, 3(3), 287-296.

Rapali, P., Garcia-Mayoral, M. F., Martinez-Moreno, M., Tarnok, K., Schlett, K., Albar, J. P., . . . Rodriguez-Crespo, I. (2011). LC8 dynein light chain (DYNLL1) binds to the C-terminal domain of ATM-interacting protein (ATMIN/ASCIZ) and regulates its subcellular localization. Biochem Biophys Res Commun, 414(3), 493-498. doi: 10.1016/j.bbrc.2011.09.093

Rapali, P., Radnai, L., Suveges, D., Harmat, V., Tolgyesi, F., Wahlgren, W. Y., . . . Pal, G. (2011). Directed evolution reveals the binding motif preference of the LC8/DYNLL hub protein and predicts large numbers of novel binders in the human proteome. PLoS One, 6(4), e18818. doi: 10.1371/journal.pone.0018818

Remenyi, A., Scholer, H. R., \& Wilmanns, M. (2004). Combinatorial control of gene expression. Nat Struct Mol Biol, 11(9), 812-815. doi: $10.1038 / \mathrm{nsmb} 820$

Scheres, S. H. (2012). RELION: implementation of a Bayesian approach to cryoEM structure determination. J Struct Biol, 180(3), 519-530. doi: 10.1016/j.jsb.2012.09.006 
Schuck, P. (2000). Size-distribution analysis of macromolecules by sedimentation velocity ultracentrifugation and lamm equation modeling. Biophys J, 78(3), 1606-1619. doi: 10.1016/S0006-3495(00)76713-0

Shammas, S. L. (2017). Mechanistic roles of protein disorder within transcription. Curr Opin Struct Biol, 42, 155-161. doi: 10.1016/j.sbi.2017.02.003

Stelter, P., Kunze, R., Flemming, D., Hopfner, D., Diepholz, M., Philippsen, P., . . . Hurt, E. (2007). Molecular basis for the functional interaction of dynein light chain with the nuclear-pore complex. Nat Cell Biol, 9(7), 788-796. doi: 10.1038/ncb1604

Svergun, D. I. (1992). Determination of the regularization parameter in indirecttransform methods using perceptual criteria. J. Appl. Cryst., 25, 495-503.

Tamiola, K., Acar, B., \& Mulder, F. A. (2010). Sequence-specific random coil chemical shifts of intrinsically disordered proteins. J Am Chem Soc, 132(51), 18000-18003. doi: 10.1021/ja105656t

Tang, G., Peng, L., Baldwin, P. R., Mann, D. S., Jiang, W., Rees, I., \& Ludtke, S. J. (2007). EMAN2: an extensible image processing suite for electron microscopy. J Struct Biol, 157(1), 38-46. doi: 10.1016/j.jsb.2006.05.009

Teufel, D. P., Bycroft, M., \& Fersht, A. R. (2009). Regulation by phosphorylation of the relative affinities of the $\mathrm{N}$-terminal transactivation domains of $\mathrm{p} 53$ for p300 domains and Mdm2. Oncogene, 28(20), 2112-2118. doi: 10.1038/onc.2009.71

Uversky, V. N. (2015). The multifaceted roles of intrinsic disorder in protein complexes. FEBS Lett, 589(19 Pt A), 2498-2506. doi: 
10.1016/j.febslet.2015.06.004

Uversky, V. N., Oldfield, C. J., Midic, U., Xie, H., Xue, B., Vucetic, S., . . Dunker,

A. K. (2009). Unfoldomics of human diseases: linking protein intrinsic disorder with diseases. BMC Genomics, 10 Suppl 1, S7. doi: 10.1186/1471-2164-10-S1-S7

Vadlamudi, R. K., Bagheri-Yarmand, R., Yang, Z., Balasenthil, S., Nguyen, D., Sahin, A. A., . . Kumar, R. (2004). Dynein light chain 1, a p21-activated kinase 1-interacting substrate, promotes cancerous phenotypes. Cancer Cell, 5(6), 575-585. doi: 10.1016/j.ccr.2004.05.022

Vistica, J., Dam, J., Balbo, A., Yikilmaz, E., Mariuzza, R. A., Rouault, T. A., \& Schuck, P. (2004). Sedimentation equilibrium analysis of protein interactions with global implicit mass conservation constraints and systematic noise decomposition. Anal Biochem, 326(2), 234-256. doi: 10.1016/j.ab.2003.12.014

Wong, D. M., Li, L., Jurado, S., King, A., Bamford, R., Wall, M., . . Heierhorst, J. (2016). The Transcription Factor ASCIZ and Its Target DYNLL1 Are Essential for the Development and Expansion of MYC-Driven B Cell Lymphoma. Cell Rep, 14(6), 1488-1499. doi: 10.1016/j.celrep.2016.01.012

Zaytseva, O., Tenis, N., Mitchell, N., Kanno, S., Yasui, A., Heierhorst, J., \& Quinn, L. M. (2014). The Novel Zinc Finger Protein dASCIZ Regulates Mitosis in Drosophila via an Essential Role in Dynein Light-Chain Expression. Genetics, 196(2), 443-453. doi: 10.1534/genetics.113.159541 


\section{Chapter 6}

\section{The Future of in situ Cryo-Electron Microscopy}

Single particle cryoEM recently evolved into a technique capable of targeting challenging macromolecular systems for high-resolution structural investigation including membrane proteins, filamentous proteins, intrinsically disordered proteins and other heterogeneous systems. The number of structures solved to near-atomic resolution by cryoEM has been rising quickly since 2013 evidencing the effectiveness of the technique. However, an important caveat remains: proteins must be removed from their native cellular environment to be studied by cryoEM (or by any other structural technique capable of reaching "near-atomic" level resolution). Removing proteins or other biological macromolecules from their native environment always brings along the question of whether or not what is visualized in isolation represents the biologically relevant structure that would be present in cells. For example, membrane proteins must be removed from their native lipid environment, and solubilized by detergents or other agents - which could induce changes in structure. Soluble proteins aren't free of problems either, since they must be removed from the crowded context of the cytosol, and may be stripped of important ligands or binding partners. In both cases, delicate macromolecular complexes can be destroyed during the purification process. Ideally, macromolecules could be imaged in their native cellular contexts; however, techniques capable of imaging in such conditions have limited resolution so far. Another limitation of imaging is that you are limited to collecting data a certain length scale for a given technique. 
For example, light microscopy can be used to identify different protein a component labeled with fluorescent tags, but is not capable of reaching the resolution that electron tomography or single particle EM can reach. Ideally, the same sample could be examined using both light microscopy and electron microscopy, so that the information collected at these different scales could be combined from the same sample. This is not currently possible.

The techniques of electron tomography, sub-tomogram averaging, and superresolution light microscopy can maintain the native cellular environment - or at least near native. Potentially, some of the technological advances that propelled single particle cryoEM could also seed a so-called resolution revolution in other fields of microscopy. For example, the same camera technology that helped single particle EM advance is now being applied to tomographic analysis. Currently, tomograms suffer from low SNR for similar reasons as single particle $\mathrm{EM}$, but to a greater degree since the dose is spread out not just over one micrograph but over a tilt series composed of many micrographs. A piece of technology that can greatly increase SNR of micrographs, the Volta phase plate, could prove helpful for such low-dose images ${ }^{1}$. Although this technology has not seen widespread use, if implemented with tomography - and sub-tomogram averaging of particles - the resolution of tomograms could be dramatically increased $^{2}$. 
The ability to combine structural data collected at different length scales would greatly expand our ability to understand cellular function at the structural level. In an ideal world, information on cell biology acquired at different length scales (and in the cellular context) could be collected from the same sample. One approach that is seeing significant effort is to combine the capabilities of super-resolution light microscopy and cryo-electron tomography, in an emerging field known as correlative-light-EM (CLEM). Here, super resolution light microscopy could be used to gather information on broad cellular context and to identify cellular components with various labeling strategies. Then, on the same specimen, CryoET could be used to collect higher resolution information on these structures. If this approach could be combined with sub-tomogram averaging, it could then be used to determine sub-nanometer resolution structures of individual cellular components or proteins, which would represent the structure in a near native context. It will be very exciting to see how technological advances in cryoEM and electron tomography will continue to pave the way towards these types of approaches. 


\section{References}

1. Danev, R., Buijsse, B., Khoshouei, M., Plitzko, J. M. \& Baumeister, W. Volta potential phase plate for in-focus phase contrast transmission electron microscopy. Proc. Natl. Acad. Sci. (2014).

doi:10.1073/pnas.1418377111

2. Khoshouei, M., Pfeffer, S., Baumeister, W., Förster, F. \& Danev, R. Subtomogram analysis using the Volta phase plate. J. Struct. Biol. (2017). doi:10.1016/j.jsb.2016.05.009 


\section{Appendix A: Full-Length Gap Junction Purification Protocol Lens Dissection}

Lysis Buffer (2 liters): 10mM Tris-8.0, 5mM EDTA, 5mM EGTA

Lenses are stored in falcon tubes at $-80 \mathrm{C}$, with $\sim 50$ lenses per 50 mLtube. First, thaw 4 tubes of previously frozen sheep lenses in an ice bath from. Starting with an intact lens, puncture the outer membrane and remove $\mathrm{w} /$ tweezers (if present - this is a black-brown capsule around the lens that is sometimes removed when the lens is dissected from the eye). Gently scrape soft cortical tissue from firm inner cortex with a scalpel, leaving the firmer core tissue. Place cortex tissue in falcon tube on ice. Store lens cores in separate tube on ice; then store tubes of dissected tissue at $-80 \mathrm{C}$

\section{Cell lysis}

Lens cortex tissue is lysed with a glass cell homogenizer. All following steps are performed on ice. Cortex cells are mixed 1:1 with lysis buffer. Perform 20 slow passes with 'loose' plunger and 20 slow passes with 'tight' plungher, or until plunging does not provide excessive resistance. Cell lysate is distributed to $6 \mathrm{x}$ $100 \mathrm{ml}$ centrifuge tubes and filled with lysis buffer. A small volume is saved for SDS-PAGE analysis. Centrifuge at $10 \mathrm{~K}$ r.p.m. $(11,000 \mathrm{xg})$ for $30 \mathrm{~min}$. at $4 \mathrm{C}$. Gently remove the supernatant by aspirating with a pipette, do not pour off the supernatant or you will loose the pellet. Resuspend each pellet with $\sim 25 \mathrm{~mL}$ of buffer, then bring up to $\sim 100 \mathrm{ml}$ lysis buffer, and centrifuge at $10 \mathrm{~K}$ r.p.m. for 30min. Repeat pellet washing previous washing step. Resuspend each pellet in 
$\sim 5 \mathrm{ml}$ 's of lysis buffer, and place pool into $50 \mathrm{~mL}$ falcon tubes as needed. Crude membrane suspension can be stored at $-20 \mathrm{C}$ or $-80 \mathrm{C}$.

\section{Cortical Membrane Preparation}

Urea Buffer (500 mL): 10mM Tris-8.0, 4M Urea, 2mM EDTA, 2mM EGTA $20 \mathrm{mM}$ $\mathrm{NaOH}(1 \mathrm{~L})$

Storage Buffer (500 mL): 10mM Tris-8.0, 2mM EDTA, 2mM EGTA

\section{Urea Wash}

Note - for each wash, ensure you save a small volume of the supernatant and of the resuspended pellet for SDS-PAGE analysis ( $20 \mathrm{uL})$. Thaw crude membrane on in an ice bath, typically $\sim 60 \mathrm{~mL}$ prepared the previous day. Dilute to $\sim 200 \mathrm{~mL}$ with urea buffer in a chilled beaker and mix well with a pipette. Centrifuge at $35 \mathrm{~K}$ r.p.m. (for Beckman rotor Ti50.2, this is $146,550 \mathrm{xg}$ ) for $30 \mathrm{~min}$. at 4C. Pour off the supernatant.

\section{$\mathrm{NaOH}$ Wash}

Resuspend the pellet in $200 \mathrm{~mL}$ of $20 \mathrm{mM} \mathrm{NaOH}$ and combine in a chilled beaker. Mix well with pipette. Centrifuge at $35 \mathrm{~K}$ r.p.m. for $30 \mathrm{~min}$. at $4 \mathrm{C}$. Note that the pellet will loose $50 \%$ mass after $\mathrm{NaOH}$ wash. Repeat the $\mathrm{NaOH}$ wash two more times for a total of 3 washes. 


\section{Storage}

Resuspend the pellet in $200 \mathrm{~mL}$ storage buffer. Remove and additional $50 \mathrm{ul}$ and determine protein concentration using a Bradford Assay. Centrifuge at 35K r.p.m. for $15 \mathrm{~min}$. at $4 \mathrm{C}$. Resuspend the pellets in appropriate volume of storage buffer to reach a final protein concentration $=2 \mathrm{mg} / \mathrm{ml}$ cortical membrane, based on the results of the Bradford Assay. Aliquot $3.5 \mathrm{~mL}$ of membrane into $15 \mathrm{ml}$ falcon tubes; wrap the lids with parafilm. Membrane can be stored at $-80 \mathrm{C}$ until needed. Check samples by SDS-PAGE (15\% gel). Load 10ul crude and wash steps; 2 ul Cx for silver stain gel.

\section{Chromatography Purification}

Solubilization: $50 \mathrm{~mL}$ stock: 10mM Tris-8.0, 2mM EDTA, 2mM EGTA, 2\% DM Ion Exchange:

1000 mL Buffer A: 10mM Tris-8.0, 2mM EDTA, 2mM EGTA, 0.3\% DM

$500 \mathrm{~mL}$ Buffer B: As buffer A, but include $2 \mathrm{M} \mathrm{NaCl}$

$500 \mathrm{~mL}$ SEC with DM: $20 \mathrm{mM}$ HEPES, pH 7.4, $150 \mathrm{mM} \mathrm{NaCl}$, 2mM EDTA, 2mM EGTA, 0.3\% DM

$250 \mathrm{~mL}$ : SEC without DM: As above, but without DM. (Note: can also be prepared with different buffer for low $\mathrm{pH}$. At low $\mathrm{pH}$, don't use aminosulfonate buffers to avoid possible interaction with gap junctions. I have used succinate and bis-tris.) 
Before beginning - note that to perform this prep as written here in a reasonable time frame, you will need access to two FPLC systems. Before beginning a full-length purification, ensure any UnoQ columns you will be using have been deep cleaned. Note that newer columns perform significantly better than older columns for full-legnth (this was observed for $1.3 \mathrm{~mL}$ columns). Ideally, columns will be set aside for full-legnth. It may also be beneficial to consider using a larger UnoQ column, which could possibly aliviate some of the issues with that column for this purfication (detailed below), but this was not tested. Note - save $20 \mathrm{uL}$ fractions from each run for gel analysis.

\section{Membrane Solubilization}

Thaw $3.5 \mathrm{ml}$ of membrane in an ice bath. For a full-scale prep targeting amphipol exchange, you may need as many as six tubes depending on your yield, but using more than six tubes is not recommended. While thawing, equilibrate the enrich 650 column, two UnoQ columns $(1.3 \mathrm{~mL})$ and the adhere column $(5 \mathrm{~mL})$. Once membrane thawed; add $3.5 \mathrm{ml}$ of solubilization buffer and incubate at $37 \mathrm{C}$ for $30 \mathrm{~min}$, with rotating. Save a sample of total membrane for SDS-PAGE analysis. Clear unsolubilized debris by centrifugation at 146,550 xg (35k rpm Ti50.2; 40k rpm in Ti70.1). Collect the supernatant and filter through a 0.22um filter tip. Save a sample of solublizied membrane and pellet for SDS-PAGE. 


\section{Anion Exchange Chromatography (Adhere and UnoQ)}

The supernatant is run over the Adhere column and the flowthrough is collected and pooled. Stop pooling once UV280 has baselined. See Figure 1 for UV280 and Figure 2 for SDS-PAGE gel. The simplest way to collect the flowthrough is by hand into a $50 \mathrm{~mL}$ falcon tube (as opposed to using the fraction collector). Run $100 \%$ buffer B to elute anything on the column and save those fractions for SDS-PAGE analysis. Do not discard until the end of your purification. The elution will contain a lot of AQP0.

Split the ADH flowthrough into two equal pools. Run both of these pools over a $1.3 \mathrm{~mL}$ unoQ column. Use two FPLC systems to run these simultaneously. There are a few issues you will want to watch for during these runs. Watch the pressure on the column - the most common issue with this purification is going over pressure on these unoQ runs (see the pressure trace on Figure 3 for an example). Ideally, run at a rate of $1.0 \mathrm{~mL} / \mathrm{min}$ and only reduce flow rate if needed to prevent overpressure. Another other common issue, usually seen with older columns, is that full-legnth connexin elutes with the AQP0 peak, and/or overlaps with the MP38 peak to a greater extent than is typically observed.

Before starting a $Q$ run, check your run setup - change the volume for loading the sample if needed. For these first $Q$ runs, use a $1.0 \mathrm{~mL}$ fraction during elution. For the FPLC run, there should be a $\sim 5 \%$ B wash after loading, and the elution step should ramp from $5 \%$ to $25 \%$ over $25 \mathrm{CV}$. The final step should be $100 \%$ B 


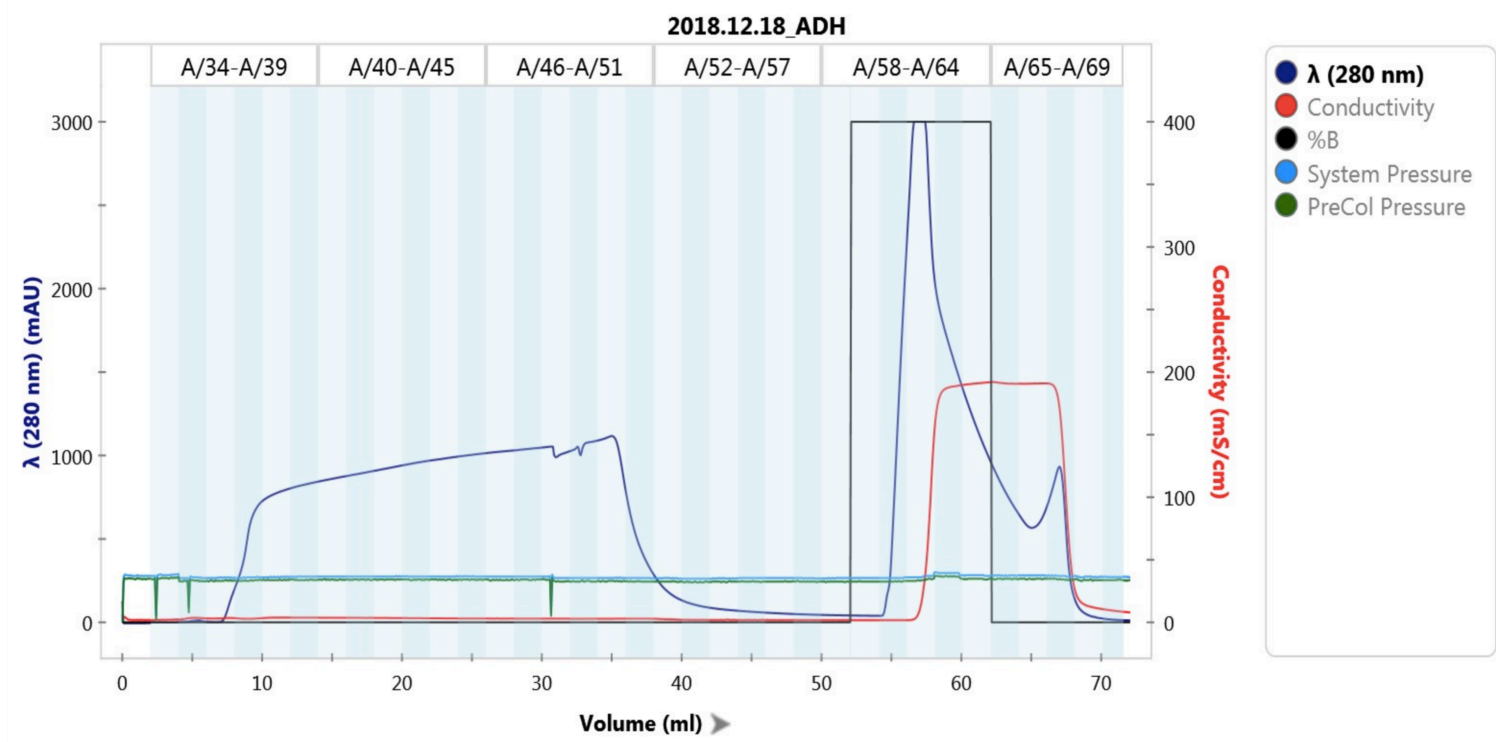

Figure 1. Adhere column trace. Full-length connexin does not bind the adhere column, so the flow-through is saved. For this trace, fractions $36-52$ were saved. The elution contains primarily aquaporin. Fractions $59-67$ were pooled and saved for SDS-PAGE analysis.

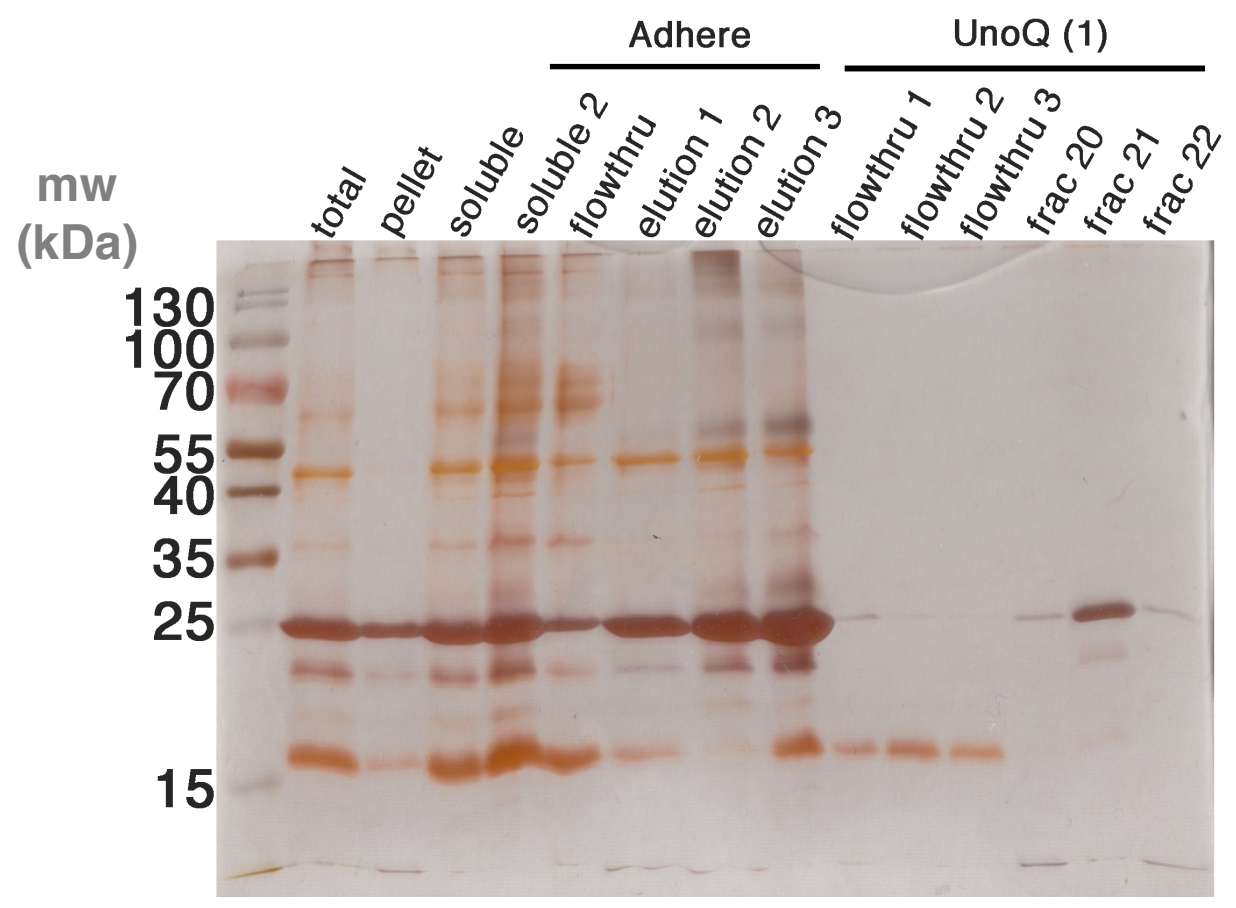

Figure 2. SDS-PAGE analysis of adhere column and supplementary UnoQ fractions. Gel shows results of detergent solubilization, adhere column, and extra uno $Q$ fractions. The adhere flowthrough was pooled for use in the next chromatography step and contains all full-legnth connexin ( 70 kDa). Cleaved cx is at $\sim 35 \mathrm{kDa}$, and aquaporin-0 at $\sim 25 \mathrm{kDa}$. 15\% acrylamide gel. 
run in reverse followed by $0 \% \mathrm{~B}$; this step cleans and re-equilibrates the column for the next run. After loading your sample, wait for the UV reading to baseline then advance the method to the next wash ( $\sim 5 \%$ buffer B). Your conductance value should be $\sim 12 \mathrm{mS} / \mathrm{cm}$ for this step. Run this step until a peak elutes and the uv280 baselines. This removes AQP0 from the column - the ADH column does not bind all of it. MP38 and full-length connexin will both elute during the gradient step, usually also with some residual AQP0. The connexin peak is the second peak, and full-length is preferentially on the right side of the peak. Try to pool fractions from the left side of the peak to enrich for full-legnth over MP38 (See Figure $\mathbf{3}$ for UV280 trace and Figure $\mathbf{4}$ for SDS-PAGE gel).

Once both intial $Q$ runs are complete, pool all the saved fractions. Dilute the sample $\sim 5 x$ with buffer $A$ to reduce salt concentraton. You will run this diluted pool back over one of the $Q$ columns. Check the method first and adjust sample load volume if needed, also, you can collect $0.5 \mathrm{~mL}$ fractions during elution to get better separation of MP38 from full-length. Pool the left side of the connexin peak and save for SEC (See Figure 5 for UV280 trace and Figure $\mathbf{6}$ for SDS-PAGE gel).

Use the SEC 650 column for SEC preferably, since this column can run faster than the S6 column. Inject up to $2 \mathrm{~mL}$ onto the SEC 650 at a flow rate of 0.7 $\mathrm{mL} / \mathrm{min}$. You may need to do two or three runs, depending on your yield. After SEC, pool all your fractions and check concentration by UV280 (see Figure 7 for 


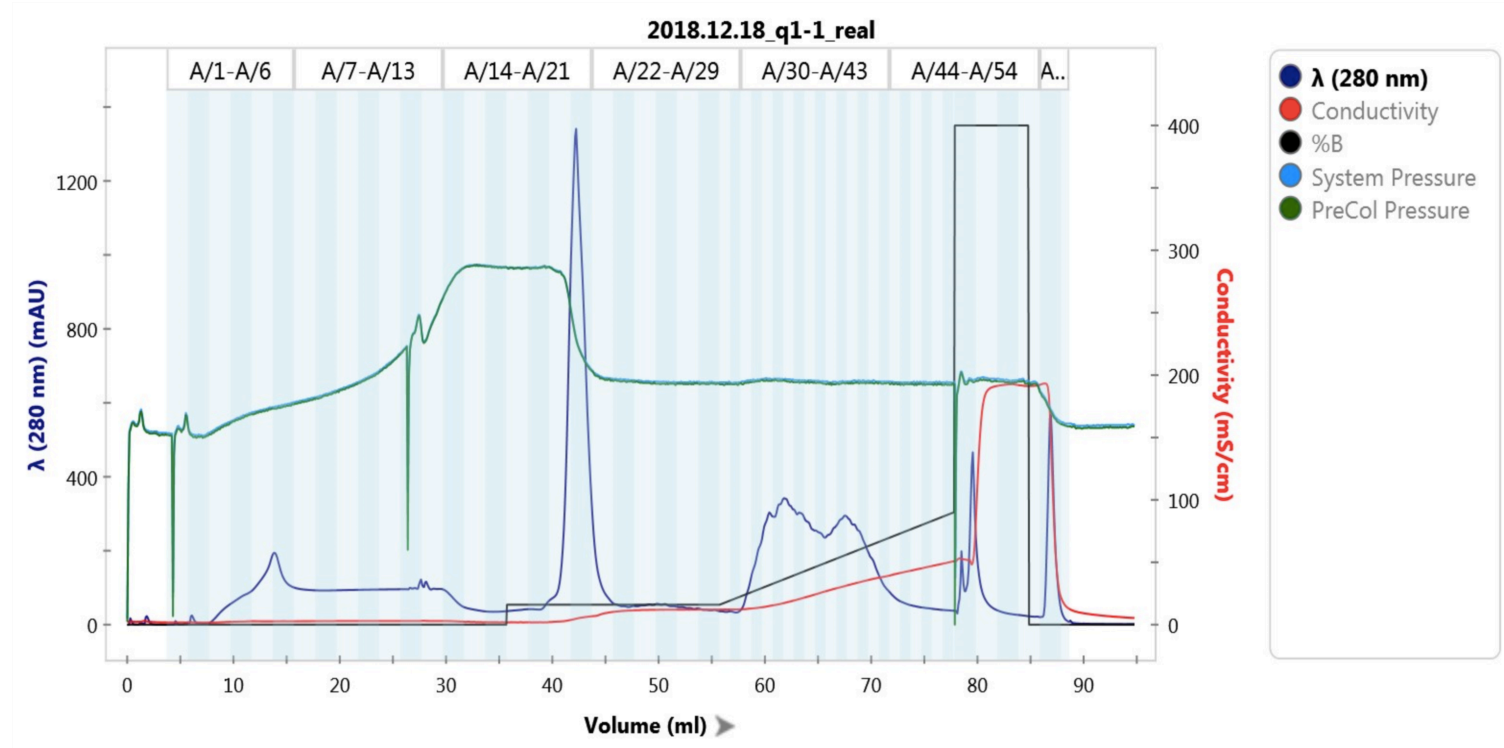

Figure 3. Initial UnoQ trace. Half of the flowthrough from the adhere column was loaded. The wash step removes aquaporin (fractions $20-22$ ). The gradient contains additional aquaporin (fractions $30-36$ ) and connexins (fractions 37- 42). The connexin peak contains both MP38 (right side) and fulllength connexin (left side, see also gel in Figure Z). For this purification, fractions 40-42 were pooled in an attempt to enrich full-length over MP38.

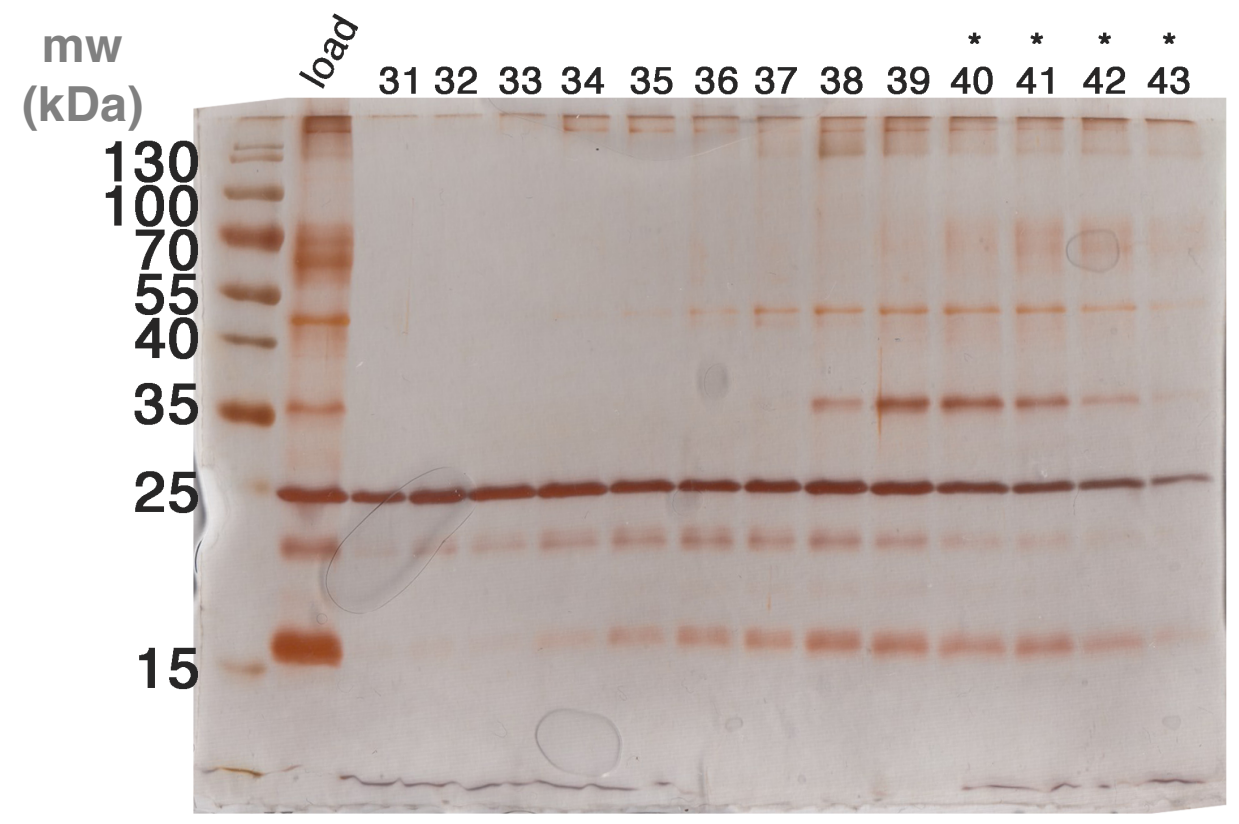

Figure 4. SDS-PAGE analysis of initial UnoQ run (UnoQ 1). Pooled fractions are marked with *. Full-length connexin is at $\sim 70 \mathrm{kDa}$, cleaved is at $\sim 35 . \mathrm{kDa}$. See Figure 2 for additional fractions. 15\% acrylamide gel. 


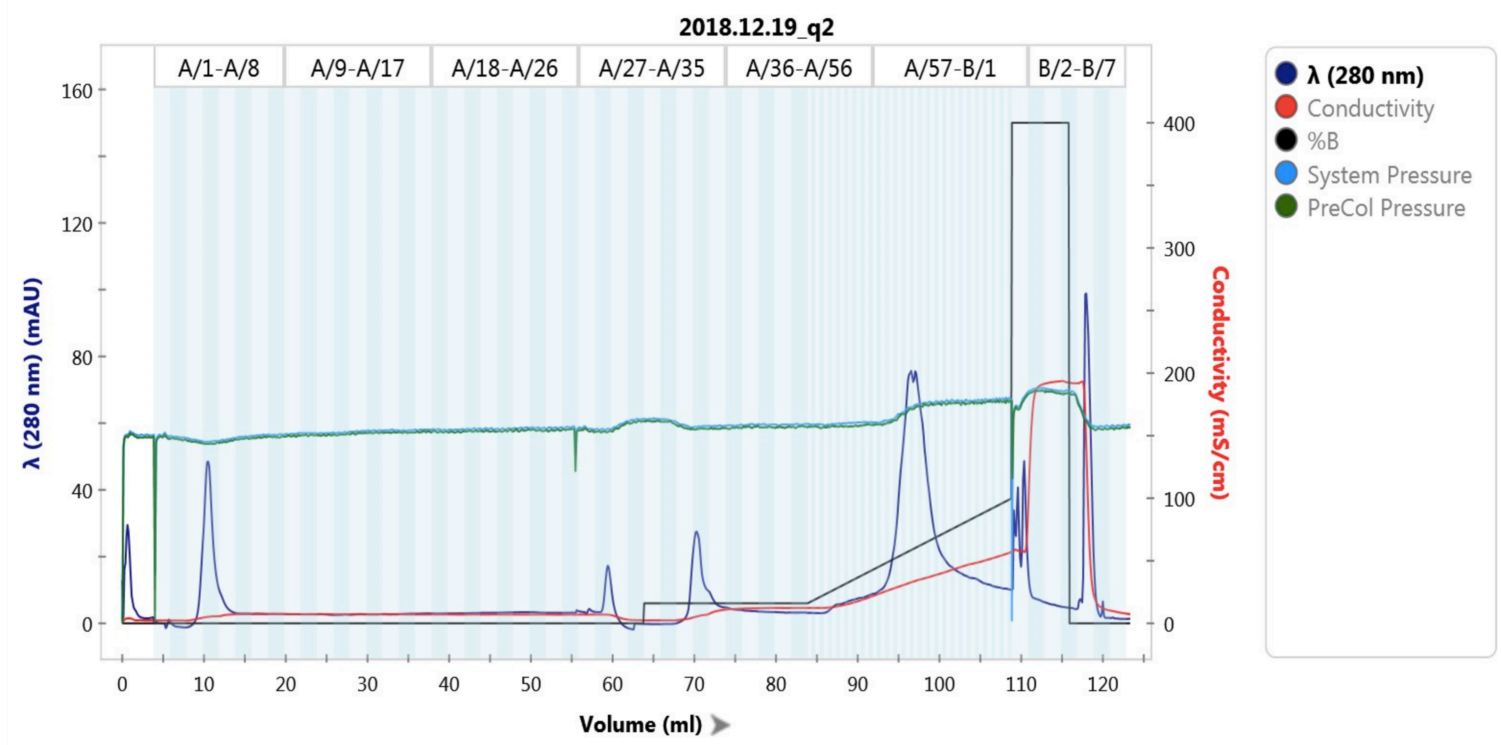

Figure 5. Final UnoQ run. Connexins are contained in fractions $57-70$. There is more MP38 on the left side of the peak and more full-lenth on the right. This trace shows an ideal situation where there is a small separation between the MP38 and full-length fractions. To enrich for full-length connexin, pool fractions from the left side of the peak (fractions $61-70$ ).

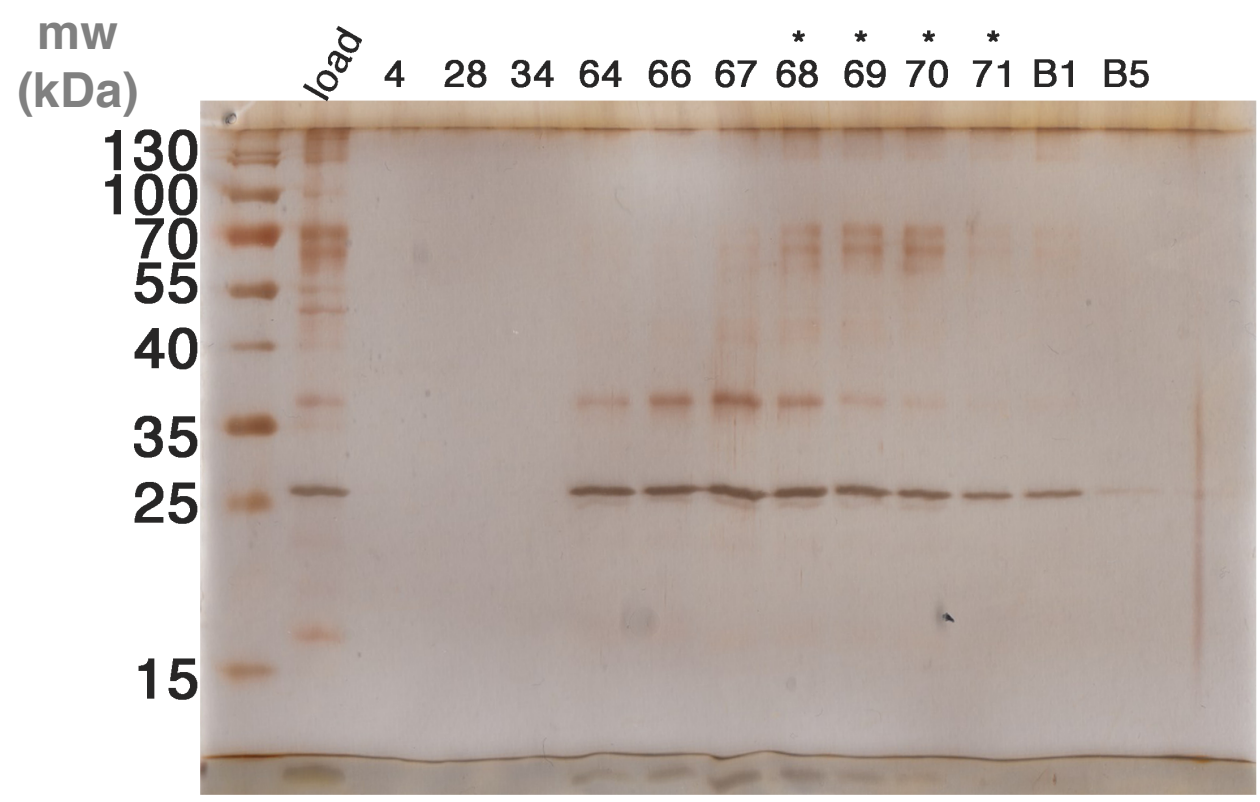

Figure 6. SDS-PAGE analysis of second UnoQ run. Load includes pool from both initial UnoQ runs. Full-length connexin is at $\sim 70 \mathrm{kDa}$, cleaved is at $35 \mathrm{kDa}$. Some separation of full-length and cleaved is achieved. $15 \%$ acrylamide gel. 


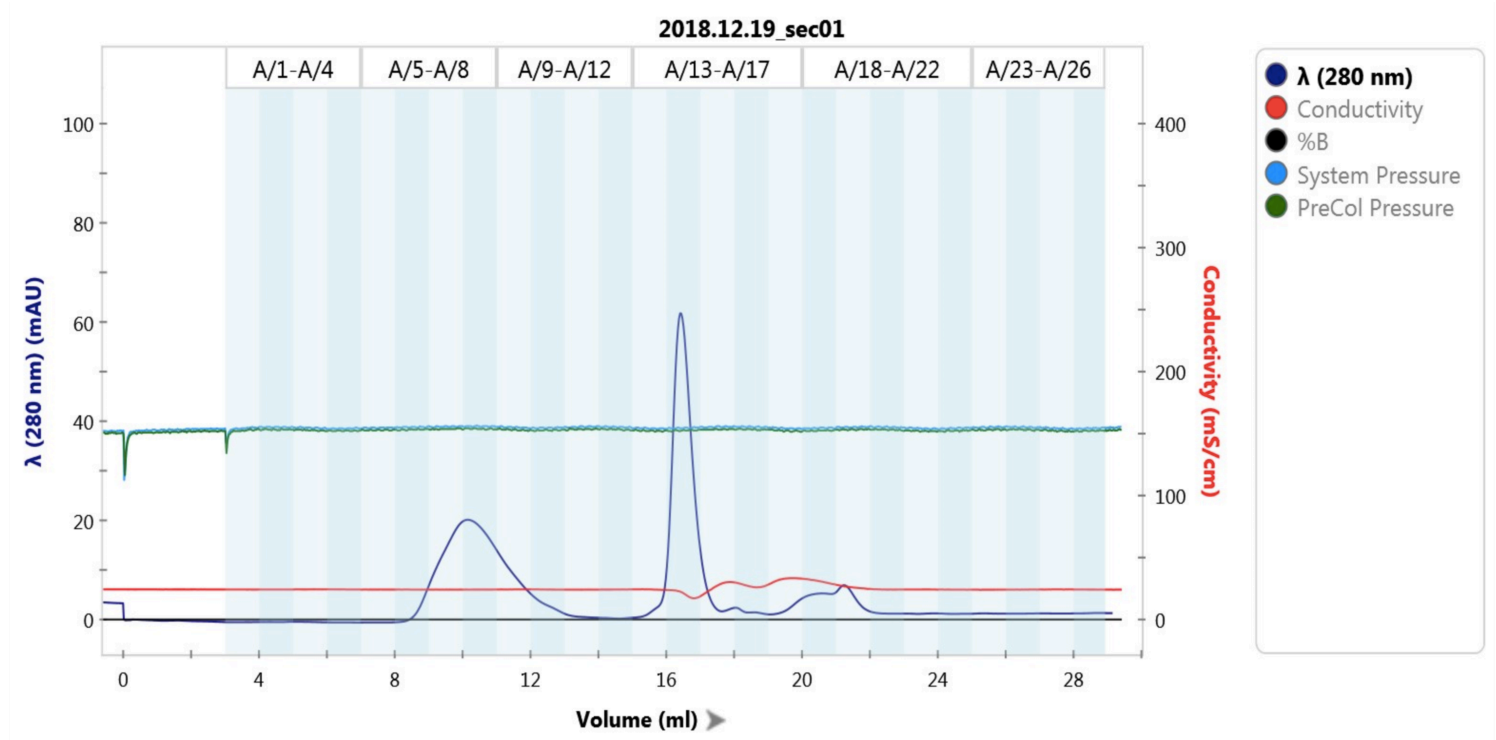

Figure 7. SEC 650 trace. Full-length connexin elutes around $10 \mathrm{~mL}$. For this run, fractions $7-8$ were pooled. Note that the additional peak at $16 \mathrm{~mL}$ is not typically seen, but this run was chosen to facilitate comparison of peak heights all from the same preparation.

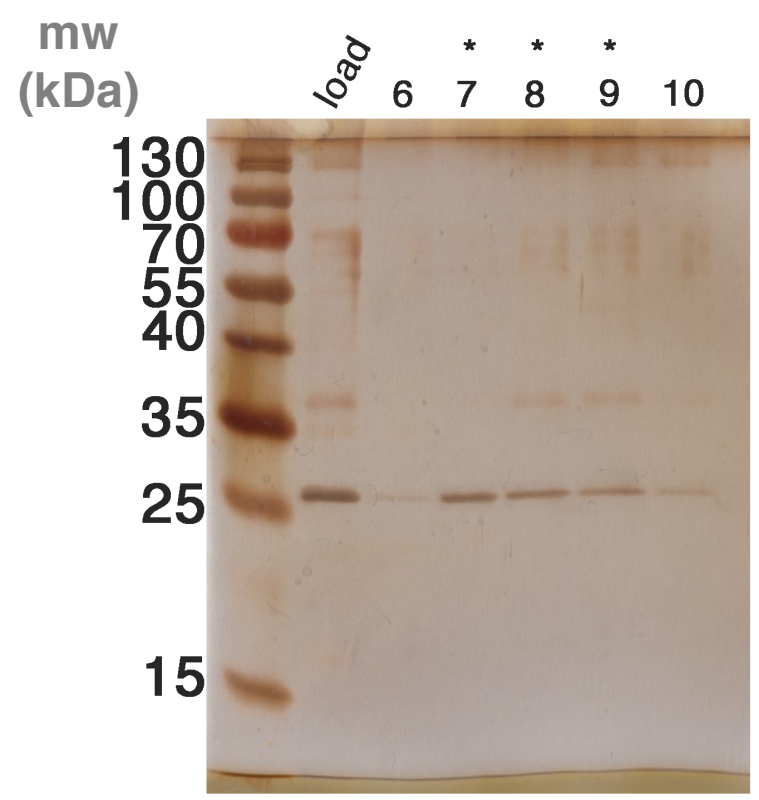

Figure 8. SDS-PAGE analysis of SEC (1 of 3 runs). Pooled fractions indicated with *. Full-length connexin at $\sim 70 \mathrm{kDa}$, cleaved at $\sim 35 \mathrm{kDa}$, aquaporin-0 at $\sim 25 \mathrm{kDa}$. $15 \%$ acrylamide gel. 


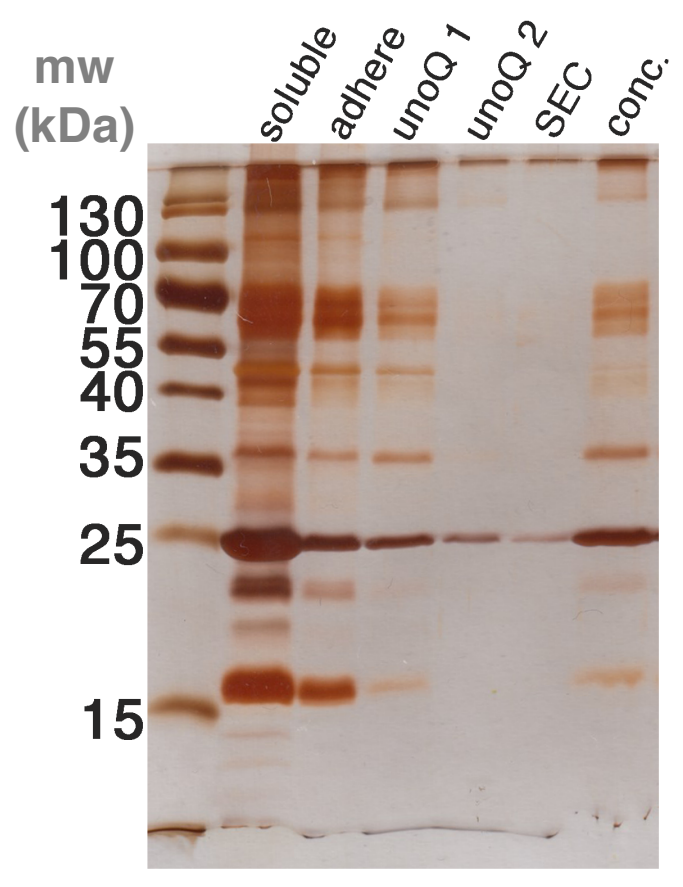

Figure 9. SDS-PAGE analysis of each purification step. Lanes contain final pool from each indicated step. Fulllength connexin at $\sim 70 \mathrm{kDa}$, cleaved at $\sim 35 \mathrm{kDa}$, aquaporin-0 at $\sim 25 \mathrm{kDa}$. UnoQ 2 and SEC did not contain enough protein to visualize. $15 \%$ acrylamide gel.

UV280 trace and Figure 8 for SDS-PAGE gel). The final pools from each purification step are compared in the SDS-PAGE gel shown in Figure 9, in order to give an overview of what each step is accomplishing.

If using amphipol A835, add amphipol in a 5:1 A835:connexin (w/w) ratio. Incubate at $4 \mathrm{C}$ for 2 hours with rotating. Try to avoid doing this in a tube that would produce a large air-water interface, it would be preferable to use many small tubes. Add biobeads in a 30:1 (w/w) ratio of beads to DM and leave overnight with rotating. Beads can be removed either by the use of a polyprep column washed with $\mathrm{dm}$ free buffer or by piercing the top of the bottom of the tube with a hot needle, then piercing the top, then spinning in a tabletop centrifuge breifly to recover the solution. Clear with an ultra centrifuge run ( $\mathrm{Ti}$ 
70.1, 40k r.p.m.,146,550 xg) and filter. Run over either the SEC 650 or enrich S6 column equilibrated with DM-free buffer, sample will elute at same volume as for DM solubilized gap junction in Figure 4. Concentrate sample in a $50 \mathrm{kDa}$ mwco concentrator at $4 \mathrm{C}$. Make negative stain grids for quality control For negative stain grids, $\sim 75 \mathrm{nM}$ particle gives good distibution. A835 recovery tended to be on the order of $20-25 \%$.

For PMAL-C8, add amphipol in a 4:1 PMAL-C8:connexin (w/w) ratio. Incubate overnight at $4 \mathrm{C}$ with rotating. Add biobeads in the same ratio as above. I had good results with leaving the sample in biobeads for 24 hours, but this is balanced by the need to leave the sample sitting for an extended period. 2-4 hours in biobeads also works. Follow the procedure above for biobead removal and cleanup. PMAL-C8 recovery for full-legnth tended to be on the order of $5 \%$ in either condition, sometimes closer to $8 \%$ with the 24 hour biobead incubation.

Nanodisk reconsitution was attempted for full-legnth, but failed. Yields were low enough that the desired lipid concentration of 4-5 mM DMPC could not be reached, which may have been part of the issue. I also realized later on that I was not adding enough biobeads to my reconstitutions. It might be beneficial to attempt this reconstiution again, if a cleaner purification could be developed. 


\section{Appendix B: Signal subtraction protocol}

To start, you will need your best 3D refinement and a PDB model of the part of the map you want to focus on (for example, a single connexin subunit). First, generate a symmetry expanded star file with relion_particle_symmetry_expand.

Use the resulting particle stack to perform a C1 refinement with a small angular search range. Use mask around the original volume. This refinement should reach a similar resolution as your non-expanded dataset - if not, something might have gone wrong during the symmetry expansion.

Next, generate a map based off the pdb in chimera using the molmap command, filtered to a low resolution. I used a single subunit volume filtered to $20 \AA$, but different resolutions should be tested to make sure you are enclosing your desired volume without creating a convoluted map. You will then need to resample the volume you created on the grid of your final map using the chimera vop command.

Generate a soft, inverted mask from this - I used a 20 pix falloff and it worked, 5 pix did not work. It is also helpful to generate a standard mask here for use in your 3D classifications.

Use relion_image_handler to multiply your map by the inverted mask - this should generate a volume for the density you wish to subtract, for example, if you 
are trying to isolate one subunit, this map should contain density for the other eleven subunits.

Create a soft mask based on the subtracted volume, also use a large soft edge on the order of 20 pix here. Once you have all of these maps and your $\mathrm{C} 1$ refined, expanded particles, run the signal subtraction job from relion.

Once it's done, you can check the subtraction by running a relion_reconstruct job, on the output. Make sure you use ctf correction. Once you have the subtracted stack you can run a 3D classification job without alignment to try and separate your populations. In relion, it has been suggested that the $\mathrm{T}$ value should be turned up to something in the range of 10-20. I used an initial classification run to remove bad particles before actually trying to separate anything out. Make sure you use more classes than the number of states you hope to resolve so that bad particles have a place to gather. When trying to separate connexin-46 from connexin-50 I used five classes.

Particles can also be imported into cisTEM, which allows the setting of a high and low-resolution limit. This could allow you to focus your refinement only on high-resolution information. 


\section{Appendix C: List of Supplemental Data}

Supplementary Movie 1: 3D reconstruction and pseudo-atomic modeling of the CaMKIla holoenzyme. Ncomms15743-s2.mov, movie file, $7.5 \mathrm{MB}$ 